University of Tennessee Health Science Center

UTHSC Digital Commons

\title{
Mild Traumatic Brain Injury with Associated Visual System Dysfunction: Investigating Histopathology, Functional Correlates, and a Novel Therapeutic Immune Modulator
}

Natalie M. Guley

University of Tennessee Health Science Center

Follow this and additional works at: https://dc.uthsc.edu/dissertations

Part of the Medical Neurobiology Commons, and the Neurosciences Commons

\section{Recommended Citation}

Guley, Natalie M. (http://orcid.org/0000-0002-4427-1799), "Mild Traumatic Brain Injury with Associated Visual System Dysfunction: Investigating Histopathology, Functional Correlates, and a Novel Therapeutic Immune Modulator" (2016). Theses and Dissertations (ETD). Paper 387. http://dx.doi.org/10.21007/ etd.cghs.2016.0406.

This Dissertation is brought to you for free and open access by the College of Graduate Health Sciences at UTHSC Digital Commons. It has been accepted for inclusion in Theses and Dissertations (ETD) by an authorized administrator of UTHSC Digital Commons. For more information, please contact jwelch30@uthsc.edu. 


\title{
Mild Traumatic Brain Injury with Associated Visual System Dysfunction: Investigating Histopathology, Functional Correlates, and a Novel Therapeutic Immune Modulator
}

\author{
Abstract \\ Background. Traumatic brain injury (TBI) is a significant source of morbidity and mortality worldwide. \\ Injuries associated with moderate to severe TBI can be profound, and have historically overshadowed the \\ significant impact mild TBI (mTBI) can have on the lives of affected individuals. Mild TBI can manifest in a \\ number of different ways, but one of the most significant and often debilitating is its impact on the visual \\ system. In order to further investigate the underlying pathology of $\mathrm{mTBI}$ and test potential therapeutics, \\ we developed a mouse model of mTBI induced by blast overpressure. In this model, a 50-60 psi blast \\ wave from a highly pressurized bolus of air is directed at a focal region of the left lateral cranium of a \\ mouse, and produces replicable motor, emotional, and visual system deficits with concomitant \\ histopathology. Importantly, this model closely simulates functional visual system damage seen in human \\ cases of mTBI. A major component of the brain's reaction to trauma is an immune response that can \\ cause additional long-term damage above and beyond that of the initial injury. This response was \\ observed in our model as regions of microglial cell activation throughout areas of the brain important for \\ visual processing. A novel therapeutic drug acting at cannabinoid type 2 receptors (CB2), known as \\ SMM-189, had previously shown promise in improving visual outcome after $\mathrm{mTBI}$ in our model, but no \\ studies were done to elucidate the cause of this improvement. The purpose of this dissertation was to \\ further characterize visual system dysfunction and histopathology in our model, as well as investigate \\ how the drug SMM-189 acts to exert its beneficial effects on these areas. Mice were blasted with either \\ 50-psi or sham blast, and then injected over the next two weeks with either drug or vehicle \\ intraperitoneally. Functional tests were administered at 30 days after blast, and perfused tissues were \\ used for subsequent histologic evaluation. Tissue used for histologic analysis was collected from mice at \\ 3 and 7 days post-blast, and in another cohort at 11 weeks after blast. \\ Functional results. Optokinetic testing was administered to obtain visual acuity (VA) and contrast \\ sensitivity (CS) thresholds in mice at 30 days after blast. It was found that no group showed any defects \\ in VA, but the 50-psi vehicle-treated group (50V) showed significant deficits in the CS function of both \\ eyes, which was completely rescued with drug treatment. Electroretinograms were run both pre- and post- \\ blast on mice to obtain an electrophysiological readout of retinal cellular function over the first month \\ after blast. The left eye of $50 \mathrm{~V}$ animals showed a pathologic B-wave elevation, but no change in the A- \\ wave, or peak latency times. Drug treatment corrected this abnormality, returning the 50-psi SMM-189 \\ treatment group (50SMM) B-wave average back to control levels.
}

Structural results. Optical coherence tomography at 30 days post-blast revealed pathologic outer retinal thinning in the left eye of $50 \mathrm{~V}$ animals, with 50SMM animals showing no such change.

Immunohistochemistry (IHC) to visualize microglia in the retina showed a significant microglial increase in the left eye of $50 \mathrm{~V}$ animals at 3 days post-blast, and a lesser but still pathologic elevation in both left and right eyes at 30 days post-blast. Drug treatment decreased the pathologic microglial elevation at both 3 days and 30 days, indicating its efficacy in quelling inflammatory microglial recruitment. Another readout of pathological response in the retina, GFAP immunoreactivity, was found to be elevated in the left eye of 50V animals at 30 days as well. 50SMM animals did not show any GFAP immunoreactivity at this time point. Brn3a+ RGCs in the retina were visualized using IHC, and no significant changes were seen. Cross-sections through optic nerves (ON) were analyzed from animals 11 weeks after blast. Left ONs from both $50 \mathrm{~V}$ and $50 \mathrm{SMM}$ animals were found to be atrophic compared to controls, while the right eyes were all equivalent. Manual axon counts revealed left ONs from $50 \mathrm{~V}$ animals had a decreased axon density, as well as a decrease in total axon count. Animals in the 50SMM group had a decreased axon 
density in the left eye, but the total axon count returned to normal. The right $\mathrm{ON}$ of $50 \mathrm{~V}$ animals also had a decrease in axon density, but the total axon count was not significantly different than controls. In the mouse brain, the right optic tract (ROT) contains predominantly the uncrossed axons originating from the left eye and optic nerve. The ROT of $50 \mathrm{~V}$ animals at 3 days after blast showed a significant number of pathologic axon bulbs, indicating areas of traumatic axonal disruption. These tracts also showed an increased presence of $\mathrm{M} 1$ inflammatory-polarized microglia when compared to controls, as determined by IHC markers specific to the M1 polarization state. In the ROT of $50 \mathrm{~V}$ animals at 5 and 7 days, large axon bulbs had decreased in number and numerous smaller granular accumulations became apparent, possibly indicating axonal degeneration. Drug treated animals showed a significant decrease in the number of axon bulbs at 3 days post-blast, and $20 \%$ of the microglia in this same tract had been converted from M1 to an M2 anti-inflammatory polarization state.

Conclusion. The novel drug SMM-189 was shown to significantly improve many aspects of visual system damage in our model. Histologic evidence supports its role in positively modulating the immune response in neurotrauma, and acting to alter microglial polarization into a more neuroprotective phenotype.

Furthermore, histologic benefits were associated with corresponding improvements in visual system function, showing its efficacy in treating $\mathrm{mTBI}$ visual system damage, a disease with no currently available pharmacotherapy. Future studies into mTBI-associated visual dysfunction should seek to investigate long-term outcome in this model, and to determine if drug benefit is sustained over an extended period after injury.

\section{Document Type}

Dissertation

\section{Degree Name}

Doctor of Philosophy (PhD)

\section{Program}

Biomedical Sciences

\section{Research Advisor}

Anton J. Reiner, Ph.D.

\section{Keywords}

Cannabinoid, Immune modulator, Microglia, Neuroimmunology, Traumatic brain injury, Visual system

\section{Subject Categories}

Medical Neurobiology | Medical Sciences | Medicine and Health Sciences | Neurosciences 
Mild Traumatic Brain Injury with Associated Visual System Dysfunction: Investigating Histopathology, Functional Correlates, and a Novel Therapeutic Immune Modulator

\author{
A Dissertation \\ Presented for \\ The Graduate Studies Council \\ The University of Tennessee \\ Health Science Center
}

\author{
In Partial Fulfillment \\ Of the Requirements for the Degree \\ Doctor of Philosophy \\ From The University of Tennessee
}

By

Natalie M. Guley

August 2016 
Portions of Chapter 3 (C) 2015 by Mary Ann Liebert, Inc. All other material (C) 2016 by Natalie M. Guley.

All rights reserved. 


\section{DEDICATION}

To my Dad, the man who believed I could do anything, and my Mom, the woman who showed me how to. 


\section{ACKNOWLEDGEMENTS}

To begin, I would like to express my profound gratitude and appreciation to my mentor Dr. Anton Reiner, without whom this dissertation would not be possible. As an undergraduate, Dr. Reiner brought me into his lab and immediately entrusted me with important projects and data, allowing me the freedom to look at things from my own perspective. Later, as a medical and then graduate student, he inspired in me a love of discovery, and constantly challenged me to learn more and investigate my own hypotheses, while guiding me always back to focus on the task at hand. Through the years, his leadership has taught me to think critically about my data, troubleshoot research problems relentlessly, and to always ask questions. With his help, I have grown into a scientist, and for that I will be forever in his debt.

I would also like to thank Dr. Marcia Honig, who has displayed immense patience in discussing countless research issues with me throughout my dissertation work, and in assisting me on confocal microscopy. Her meticulous and thoughtful examination of both histology and immunohistochemistry has taught me to never overlook the minute details of my work, because in them, you can often find the truth.

To my committee members Dr. Scott Heldt, Dr. Monica Jablonski, and Dr. Bob Moore, I can't thank you enough for all of the insightful discussion and critiques throughout the process of planning and running my dissertation experiments. A special thanks is also due to Dr. Bob Moore for the many long hours he spent synthesizing the experimental drug that played such an important role in my research. I owe my gratitude to Dr. Wei Bu, Dr. Nobel Del Mar, Dr. Chunyan Li, and Dr. Malinda Fitzgerald for so much help on a day-to-day basis with running experiments, learning new techniques, discussing issues, and scholarly debate. A very special thanks is owed to Marion Joni, who hates to be called the lab manager, and so instead I will simply call her the lab rock. She has helped me in so many ways, I can not even enumerate them. Suffice to say, much of the work in this dissertation would have been impossible without her. I have rarely seen a more hard-working and steadfast individual.

Dr. Chaela Presley and Dr. Ammaar Abidi, you graciously set aside your own work to prepare for me the experimental drug, often with very little notice, and I truly appreciate that. Additionally, I thank Chaela for the excellent chemokine and cytokine assay figures used in Chapter 2. I am indebted to Josh Rogers, Lauren D’Surney, Ting Wong, and Jessica Ferrell for all of their hard work running behavioral and vision tests on animals, performing histologic processing and analysis, and generally for their friendship. Also to Dr. Jeffery Marchetta at the University of Memphis, whose engineering experience and guidance has been integral for the creation and optimization of our blast device. Dr. John Varriano at Christian Brothers University, I appreciate not only your excellent physics teaching, but your input on the biomechanics of our model. A very heartfelt thank you to the Gangaraju lab for training on retinal angiography, Dr. T.J. Hollingsworth, and Dr. Amanda Preston for their constant help on confocal microscopy 
and retinal processing, and to the many, many people who have helped me along the way and that I am regrettably forgetting.

A special thanks is in order to Dr. Pat Ryan and Dr. Jay Callaway for helping in any way they could to coordinate and facilitate my progress through the $\mathrm{MD} / \mathrm{PhD}$ program. Many hurdles were removed from my path with their gracious assistance. I would also like to extend my most sincere appreciation the UTHSC College of Graduate Health Sciences and the UTHSC Neuroscience Institute for providing the generous funding that has allowed me to pursue this degree.

Arguably the most important recognitions are due to my parents Michael and Sally, and my siblings Jessica, Lara, and Michael. You helped mold me into the person I am, and have provided unwavering support and assistance through my entire journey. No words can convey my gratitude. Also to my beautiful and intelligent daughter Elowyn, you are too young to read this now but you have brought me joy and grounded me through some of the toughest days. You have also frustrated me to tears, a feat your grandfather would be proud of. To my best friends, Erin Gooch, Jordan Toler, and Dr. Brienne Petcher. You women are beautiful and amazing, and I am honored that you are a part of my life. All of these people have graced my life with so many blessings, it is truly unbelievable to reflect on.

Lastly, to my husband, Dr. Patrick Guley, the most analytical and thoroughly skeptical scientist I have ever met. Your incessant love of debate has often challenged my patience, while simultaneously expanding my mind to explore possibilities and topics I would never have previously considered. I cannot thank you enough for the countless hours you've spent discussing my research, giving opinions and suggestions, and critiquing conclusions at every step of the way. When we joined our hands in marriage, we vowed to always pursue the truth, and I could not have asked for a better partner in exploring every inch of this wondrous cosmos. I love you with all of my being. 


\begin{abstract}
Background. Traumatic brain injury (TBI) is a significant source of morbidity and mortality worldwide. Injuries associated with moderate to severe TBI can be profound, and have historically overshadowed the significant impact mild TBI (mTBI) can have on the lives of affected individuals. Mild TBI can manifest in a number of different ways, but one of the most significant and often debilitating is its impact on the visual system. In order to further investigate the underlying pathology of mTBI and test potential therapeutics, we developed a mouse model of mTBI induced by blast overpressure. In this model, a 50-60 psi blast wave from a highly pressurized bolus of air is directed at a focal region of the left lateral cranium of a mouse, and produces replicable motor, emotional, and visual system deficits with concomitant histopathology. Importantly, this model closely simulates functional visual system damage seen in human cases of mTBI. A major component of the brain's reaction to trauma is an immune response that can cause additional long-term damage above and beyond that of the initial injury. This response was observed in our model as regions of microglial cell activation throughout areas of the brain important for visual processing. A novel therapeutic drug acting at cannabinoid type 2 receptors (CB2), known as SMM-189, had previously shown promise in improving visual outcome after mTBI in our model, but no studies were done to elucidate the cause of this improvement. The purpose of this dissertation was to further characterize visual system dysfunction and histopathology in our model, as well as investigate how the drug SMM-189 acts to exert its beneficial effects on these areas. Mice were blasted with either 50-psi or sham blast, and then injected over the next two weeks with either drug or vehicle intraperitoneally. Functional tests were administered at 30 days after blast, and perfused tissues were used for subsequent histologic evaluation. Tissue used for histologic analysis was collected from mice at 3 and 7 days post-blast, and in another cohort at 11 weeks after blast.
\end{abstract}

Functional results. Optokinetic testing was administered to obtain visual acuity (VA) and contrast sensitivity (CS) thresholds in mice at 30 days after blast. It was found that no group showed any defects in VA, but the 50-psi vehicle-treated group (50V) showed significant deficits in the CS function of both eyes, which was completely rescued with drug treatment. Electroretinograms were run both pre- and post-blast on mice to obtain an electrophysiological readout of retinal cellular function over the first month after blast. The left eye of $50 \mathrm{~V}$ animals showed a pathologic B-wave elevation, but no change in the A-wave, or peak latency times. Drug treatment corrected this abnormality, returning the 50-psi SMM-189 treatment group (50SMM) B-wave average back to control levels.

Structural results. Optical coherence tomography at 30 days post-blast revealed pathologic outer retinal thinning in the left eye of 50V animals, with 50SMM animals showing no such change. Immunohistochemistry (IHC) to visualize microglia in the retina showed a significant microglial increase in the left eye of $50 \mathrm{~V}$ animals at 3 days post-blast, and a lesser but still pathologic elevation in both left and right eyes at 30 days post-blast. Drug treatment decreased the pathologic microglial elevation at both 3 days and 30 days, indicating its efficacy in quelling inflammatory microglial recruitment. 
Another readout of pathological response in the retina, GFAP immunoreactivity, was found to be elevated in the left eye of 50V animals at 30 days as well. 50SMM animals did not show any GFAP immunoreactivity at this time point. Brn3a+ RGCs in the retina were visualized using IHC, and no significant changes were seen. Cross-sections through optic nerves $(\mathrm{ON})$ were analyzed from animals 11 weeks after blast. Left ONs from both $50 \mathrm{~V}$ and 50SMM animals were found to be atrophic compared to controls, while the right eyes were all equivalent. Manual axon counts revealed left ONs from 50V animals had a decreased axon density, as well as a decrease in total axon count. Animals in the 50SMM group had a decreased axon density in the left eye, but the total axon count returned to normal. The right $\mathrm{ON}$ of $50 \mathrm{~V}$ animals also had a decrease in axon density, but the total axon count was not significantly different than controls. In the mouse brain, the right optic tract (ROT) contains predominantly the uncrossed axons originating from the left eye and optic nerve. The ROT of $50 \mathrm{~V}$ animals at 3 days after blast showed a significant number of pathologic axon bulbs, indicating areas of traumatic axonal disruption. These tracts also showed an increased presence of M1 inflammatory-polarized microglia when compared to controls, as determined by IHC markers specific to the M1 polarization state. In the ROT of 50V animals at 5 and 7 days, large axon bulbs had decreased in number and numerous smaller granular accumulations became apparent, possibly indicating axonal degeneration. Drug treated animals showed a significant decrease in the number of axon bulbs at 3 days post-blast, and $20 \%$ of the microglia in this same tract had been converted from M1 to an M2 anti-inflammatory polarization state.

Conclusion. The novel drug SMM-189 was shown to significantly improve many aspects of visual system damage in our model. Histologic evidence supports its role in positively modulating the immune response in neurotrauma, and acting to alter microglial polarization into a more neuroprotective phenotype. Furthermore, histologic benefits were associated with corresponding improvements in visual system function, showing its efficacy in treating mTBI visual system damage, a disease with no currently available pharmacotherapy. Future studies into mTBI-associated visual dysfunction should seek to investigate long-term outcome in this model, and to determine if drug benefit is sustained over an extended period after injury. 


\section{TABLE OF CONTENTS}

CHAPTER 1. INTRODUCTION AND BACKGROUND ...............................................1

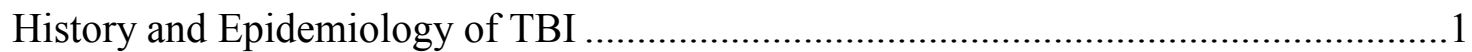

Consequences of TBI.......................................................................................

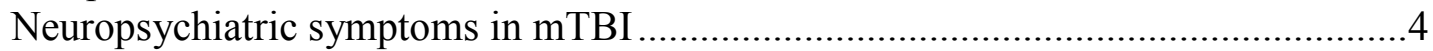

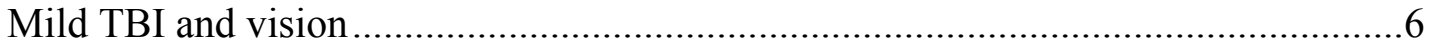

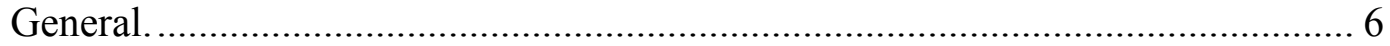

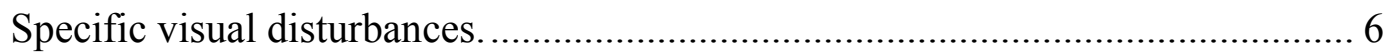

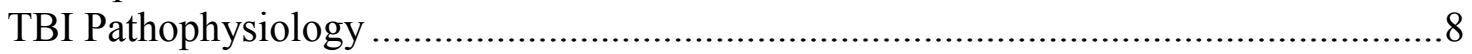

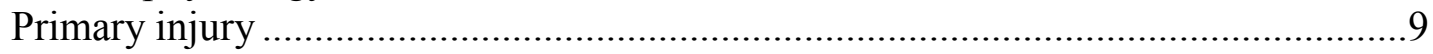

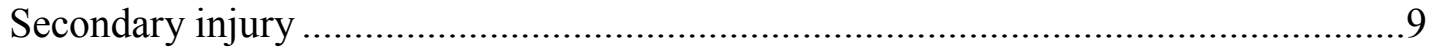

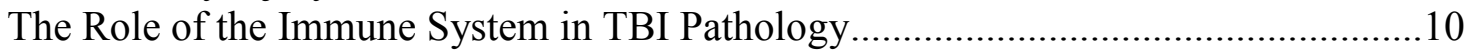

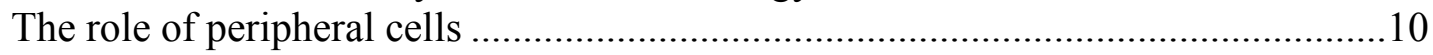

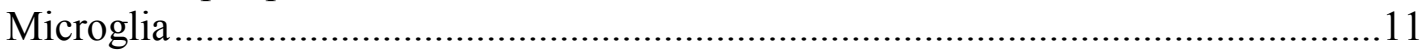

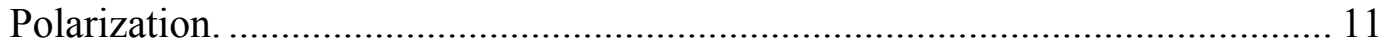

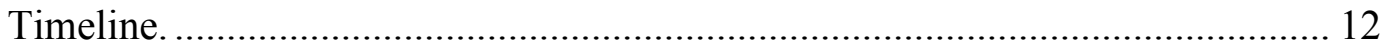

Animal Models Used in TBI Research ............................................................... 12

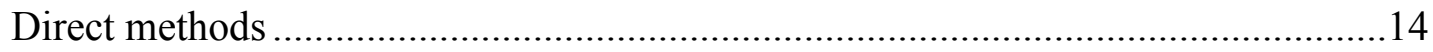

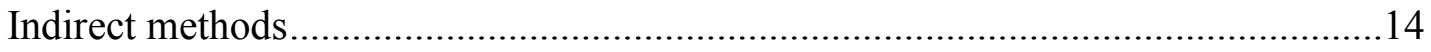

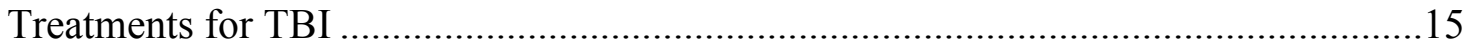

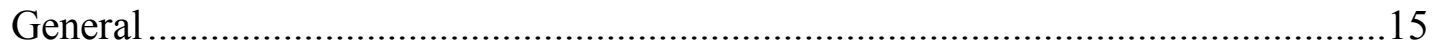

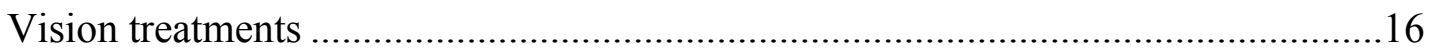

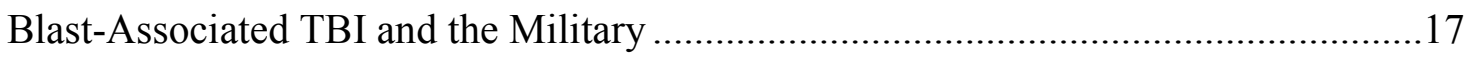

\section{CHAPTER 2. PRIOR PUBLISHED STUDIES ON A MOUSE MODEL OF} MTBI AND THE DRUG SMM-189 ................................................................18

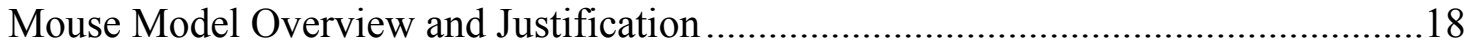

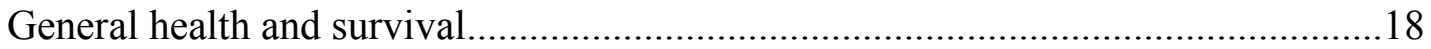

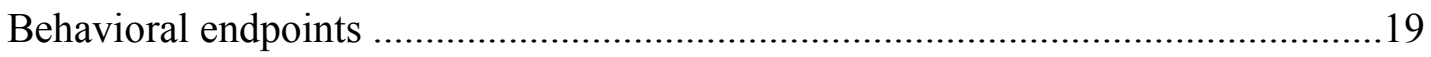

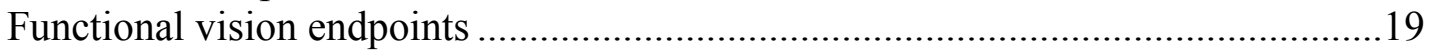

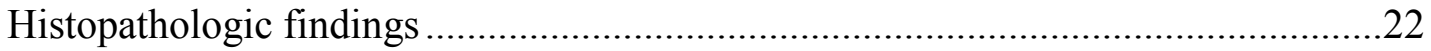

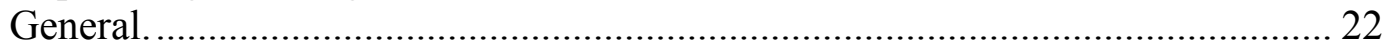

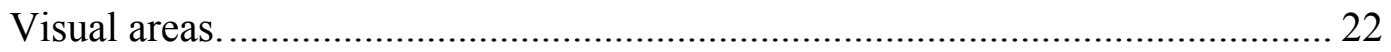

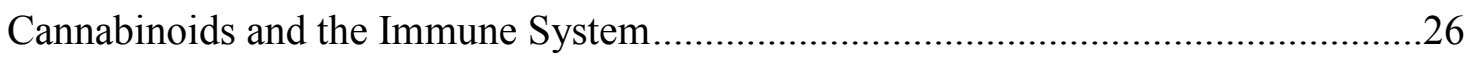

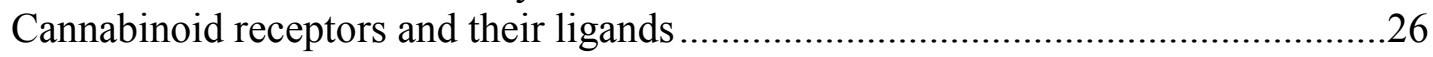

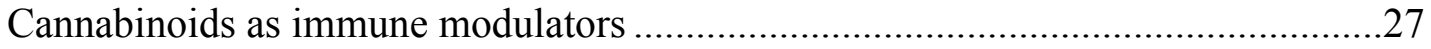

Investigative drug SMM-189, an Inverse Agonist at CB2 Receptors: Evidence for

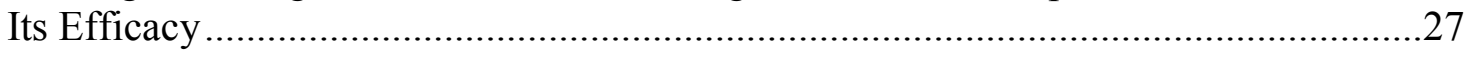

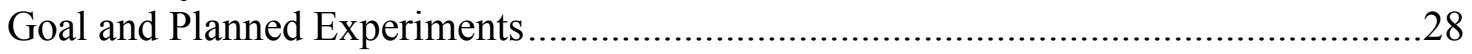

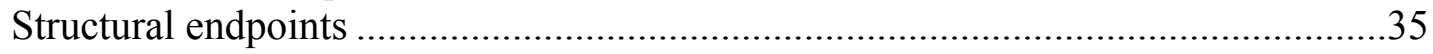

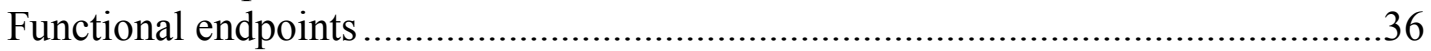




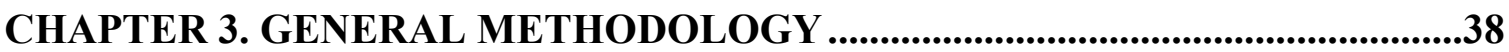

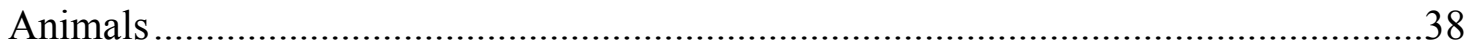

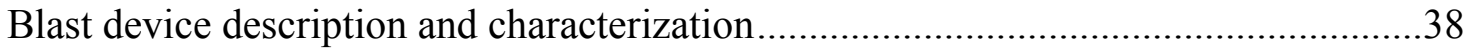

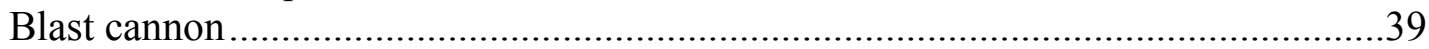

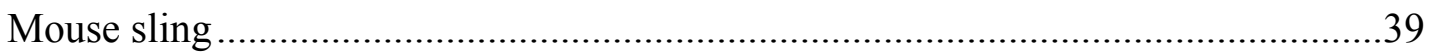

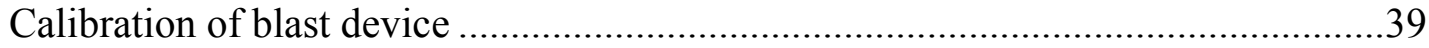

Administration of Single Overpressure Blast and Post-Blast Recovery.........................40

SMM-189 and Vehicle Administration ....................................................................40

Administration of 4x Multiple Overpressure Blast and Post-Blast Recovery ................41

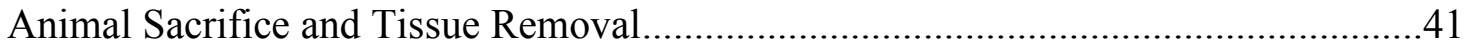

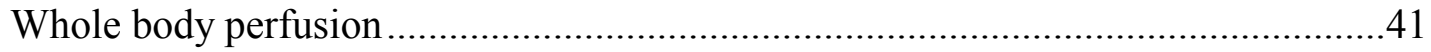

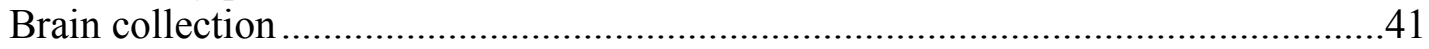

Eye and optic nerve collection .............................................................................

CHAPTER 4. FUNCTIONAL VISION OUTCOMES IN MTBI ................................43

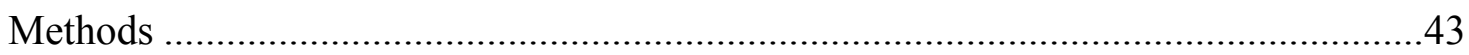

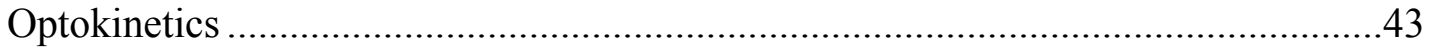

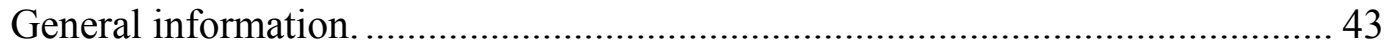

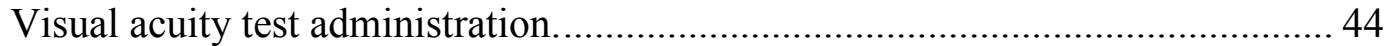

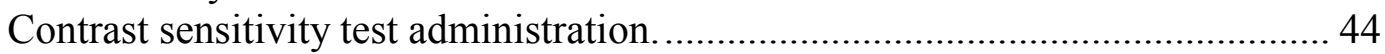

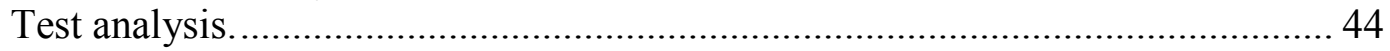

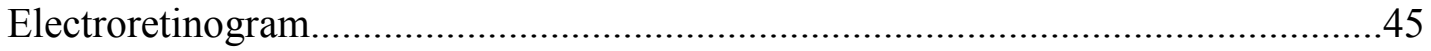

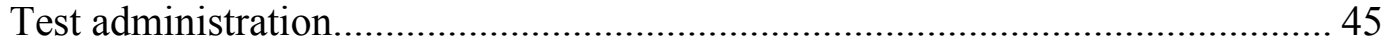

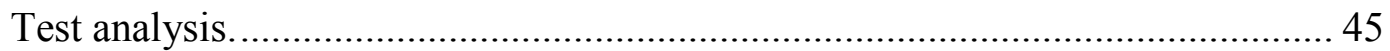

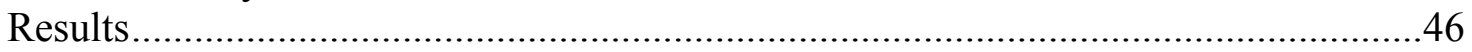

OKN

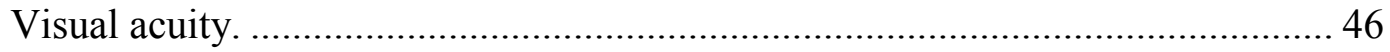

Contrast sensitivity................................................................................. 46

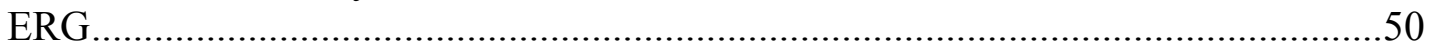

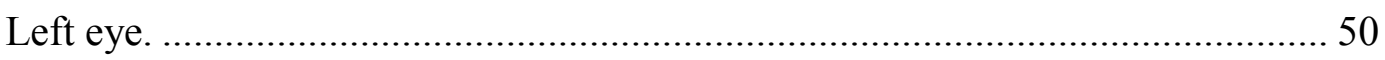

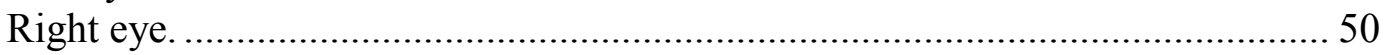

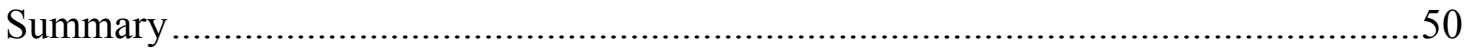

CHAPTER 5. STRUCTURAL VISION OUTCOMES IN MTBI..................................56

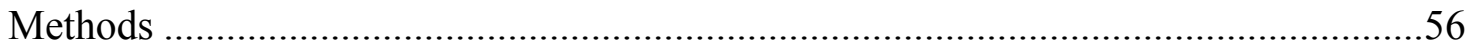

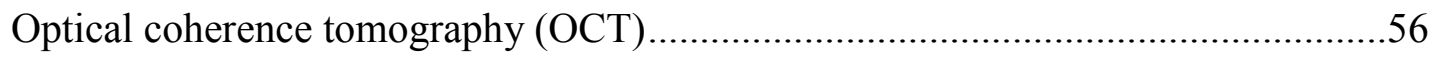

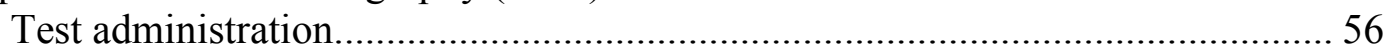

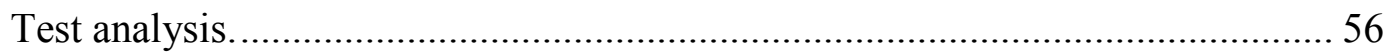

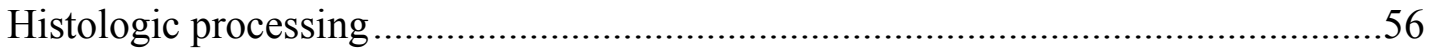

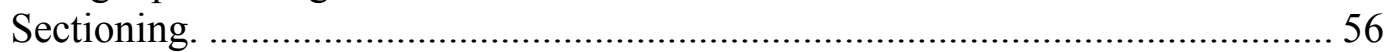

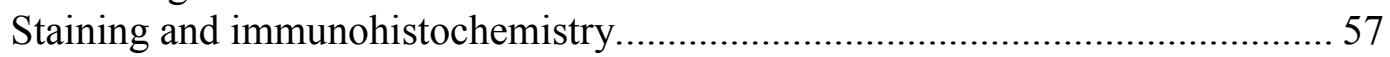

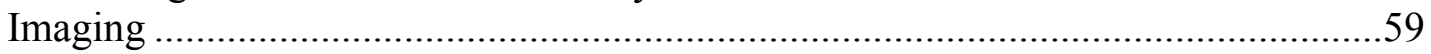

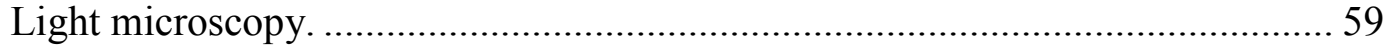

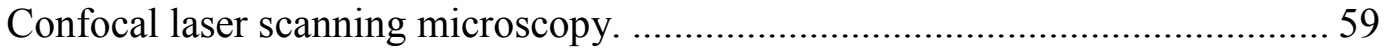

Quantification and analysis of structural end points .................................................59 
Axons in the optic nerve (LM) ....................................................................... 59

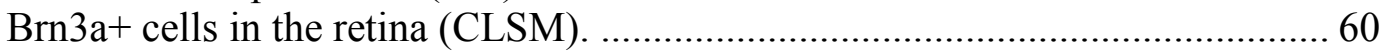

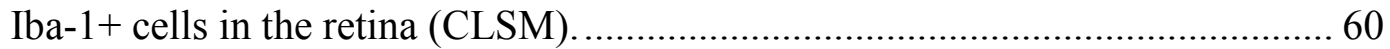

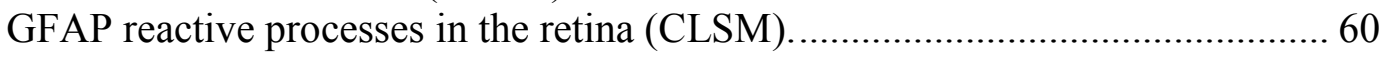

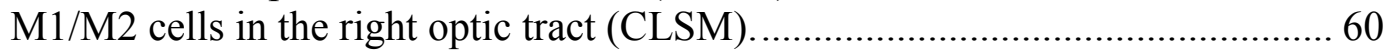

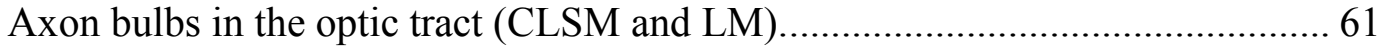

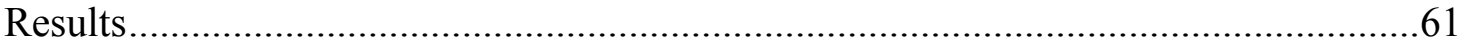

OCT

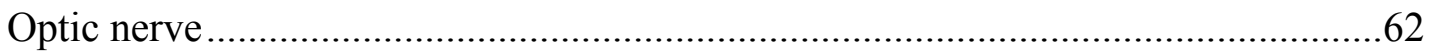

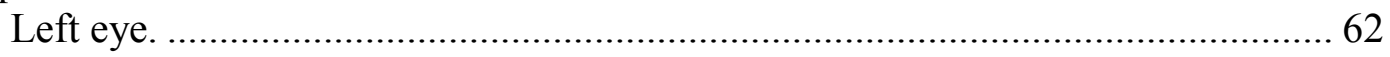

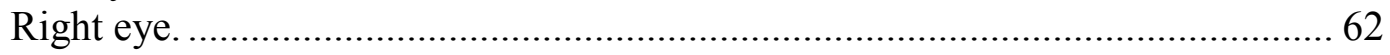

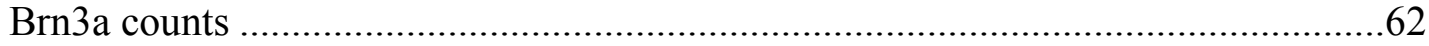

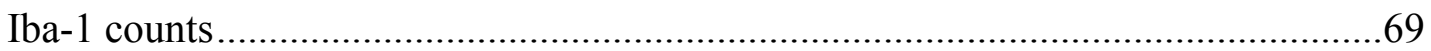

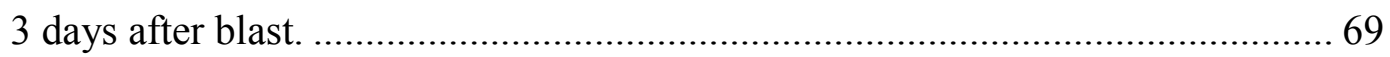

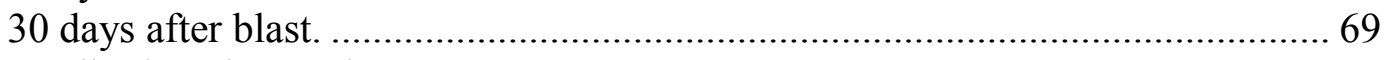

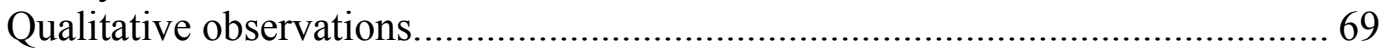

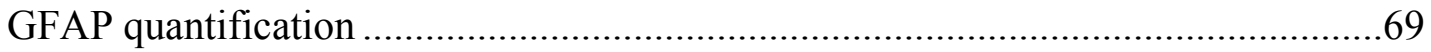

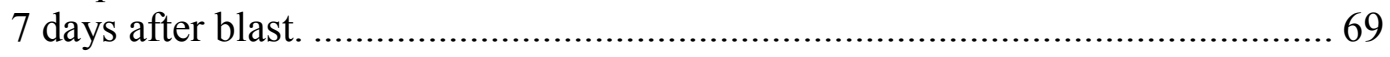

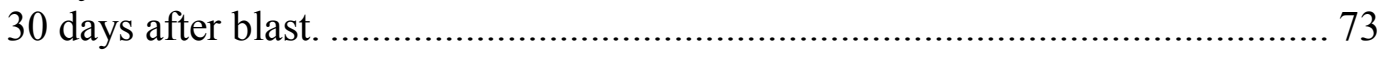

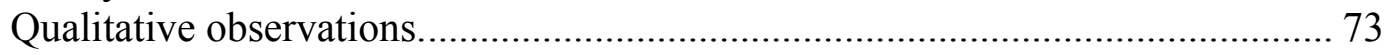

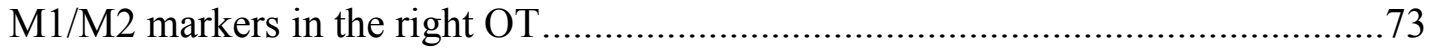

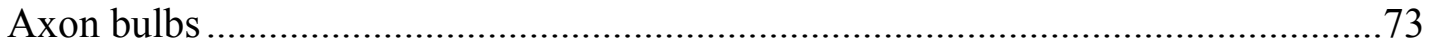

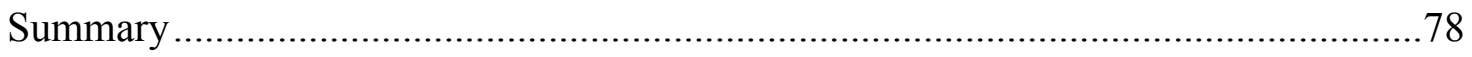

CHAPTER 6. DISCUSSION ........................................................................................81

The Elucidated Histopathology of mTBI-Associated Visual Dysfunction ....................81

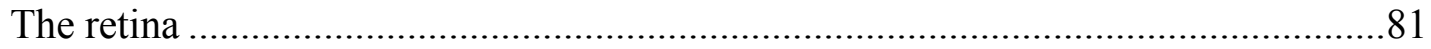

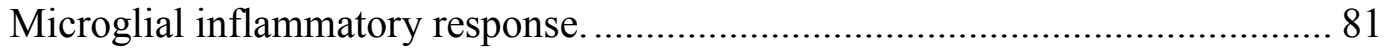

Müller glial cell response and retinal ganglion cell survival. ................................ 85

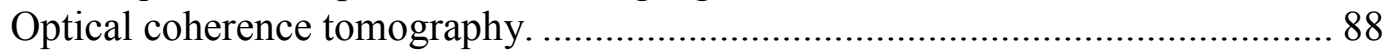

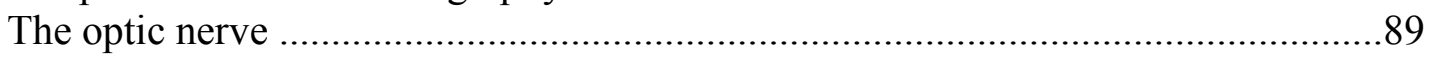

Quantitative assessments: Axon counts............................................................. 89

Qualitative assessments from IHC.............................................................. 92

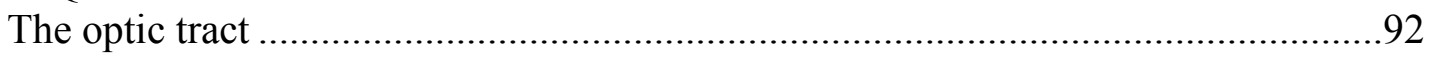

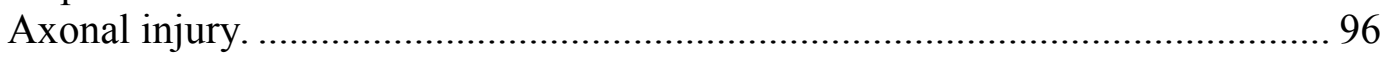

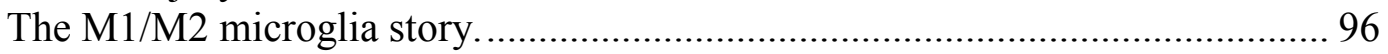

Functional Visual Tests and Their Relevance to Histologic Findings..........................102

The ERG B wave increase: Discussions of a Seemingly Paradoxical Finding ......102

Contributors to the ERG waveform: Evidence from the literature..................... 103

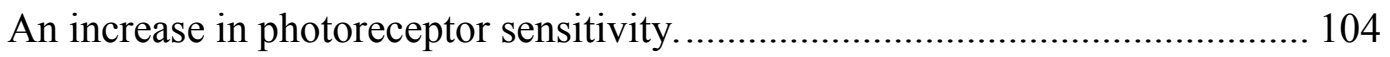

Rod-driven on-bipolar cells and loss of lateral inhibition. ................................. 105

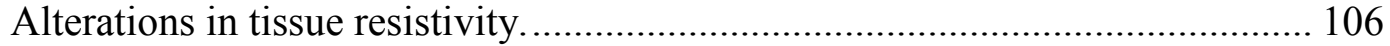

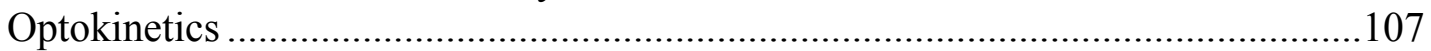

The pathways involved in the OKN and optomotor response............................ 108

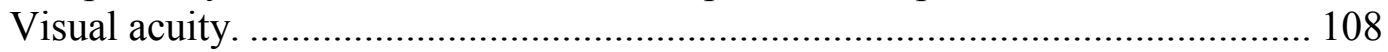

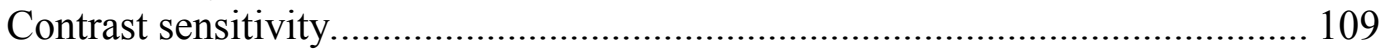


Future research strategies................................................................. 110

A Discussion of Injury Mechanisms and Inconsistencies .......................................111

Axonal shearing and stretching due to head, eye, and brain movement .................111

The problem of the left eye-right eye difference ...............................................112

SMM-189 Benefit and Implications Going Forward ............................................113

The complicated issue of microglial polarization ..............................................113

Cannabinoids as immune modulators: Issues, benefits, complicating factors ........114

CB2 receptor tissue distribution.................................................................... 114

Cannabinoids and their receptors: Beyond CB1 and CB2 ........................... 115

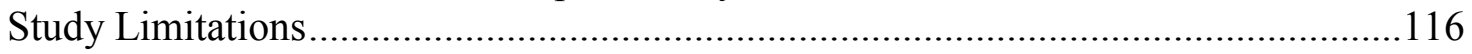

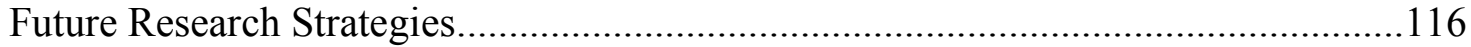

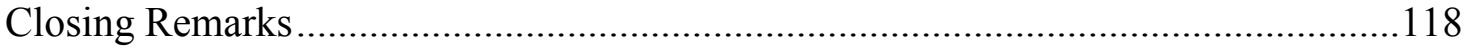

LIST OF REFERENCES .............................................................................119

VITA 


\section{LIST OF TABLES}

Table 1-1. Department of Defense traumatic brain injury classification criterion...........2

Table 1-2. $\quad$ Health effects associated with TBI. ...........................................................

Table 2-1. Summary of previous SMM-189 findings in our mouse model of TBI. ......33

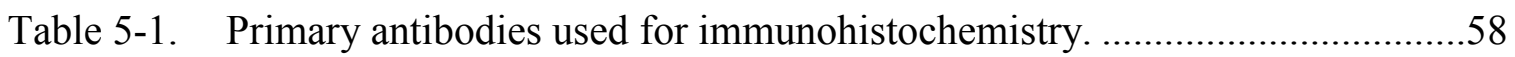

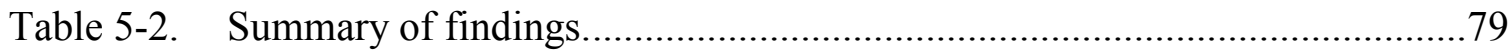




\section{LIST OF FIGURES}

Figure 1-1. In vivo models of traumatic brain injury. ………………….....................13

Figure 2-1. The effect of blast on motor performance..................................................20

Figure 2-2. The effect of blast on the visual system.................................................23

Figure 2-3. Iba-1 immunostaining in the brain of blasted animals................................25

Figure 2-4. Cytokine assay profiles of human microglia in vitro....................................29

Figure 2-5. Chemokine assay profiles of human microglia in vitro.................................30

Figure 2-6. Cell surface expression of M1 markers on human microglia in vitro............31

Figure 2-7. Cell surface expression of M2 markers on human microglia in vitro............32

Figure 2-8. The effect of SMM-189 treatment on visual end points.................................34

Figure 4-1. Visual acuity thresholds for both eyes at 30 days after blast.........................47

Figure 4-2. Visual acuity thresholds for $4 \mathrm{x}$ multi-blast animals a year after blast..........48

Figure 4-3. Contrast sensitivity functions of the left eye at 30 days after blast. .............49

Figure 4-4. Contrast sensitivity functions of the right eye at 30 days after blast. ...........51

Figure 4-5. Contrast sensitivity in $4 \mathrm{x}$ multi-blast animals a year from blast...................52

Figure 4-6. The effect of blast and SMM-189 treatment on ERG A- and B-wave functions at 30 days after blast. ................................................................53

Figure 4-7. Left eye pre-blast and 30 days post-blast scotopic ERG B-wave

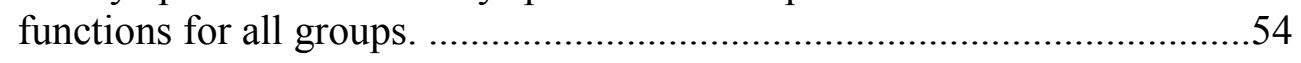

Figure 5-1. Left eye pre-blast and post-blast OCT measurements.................................63

Figure 5-2. Right eye pre-blast and post-blast OCT measurements................................64

Figure 5-3. Optic nerve cross-sectional area at 11 weeks after blast................................65

Figure 5-4. Axon density in the optic nerve at 11 weeks after blast. ..............................66

Figure 5-5. Total axons in the optic nerve 11 weeks after blast.......................................67

Figure 5-6. Brn3a+ retinal ganglion cell counts in the retina at 30 days after blast........68 
Figure 5-7. Microglial cell counts in the retina at 3 days after blast. ........................... 70

Figure 5-8. Microglial cell counts in the retina at 30 days after blast. ..........................71

Figure 5-9. Müller cell glial reactivity in the retina at 7 days after blast. ......................72

Figure 5-10. Müller cell glial reactivity in the retina at 30 days after blast. ...................74

Figure 5-11. Microglial polarization in the right optic tract at 3 days after blast.............75

Figure 5-12. Axon bulbs in the right optic tract at 3 days after blast. ............................. 76

Figure 5-13. The number of axon bulbs in the right optic tract over the first week

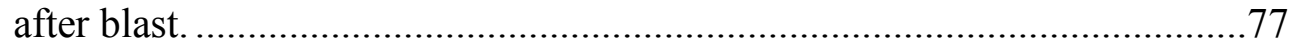

Figure 6-1. Microglia in the retina at 3 days after blast............................................83

Figure 6-2. Microglial morphology in the retina at 30 days after blast. ........................84

Figure 6-3. Müller glial morphology in the retina at 30 days after blast.......................86

Figure 6-4. High power cross sections through the optic nerve to visualize and quantify axons at 11 weeks after blast.

Figure 6-5. Scatterplot of optic nerve axon density versus optic nerve cross sectional area at 11 weeks after blast.

Figure 6-6. Histologic evidence for a focal optic nerve injury in a 3 day post-blast animal.

Figure 6-7. Hypothesized left eye injury mechanics in the moments directly after blast.

Figure 6-8. Alterations in the glial meshwork of the left optic nerve at 30 days after blast. .95

Figure 6-9. Axon bulbs in the right optic tract at 3 days post-blast. . .97

Figure 6-10. Microglial morphology and polarization markers in the right optic tract.....99

Figure 6-11. Microglial polarization comparison chart...... 100

Figure 6-12. Scatterplot of axon bulbs versus number of M2-biased microglia. 101 


\section{LIST OF ABBREVIATIONS}

\begin{tabular}{|c|c|}
\hline AEA & N-arachidonoylethanolamide \\
\hline A-D & Acceleration-deceleration injury \\
\hline AOS & Accessory optic system \\
\hline APC & Antigen presenting cell \\
\hline ATP & Adenosine triphosphate \\
\hline $\mathrm{BBB}$ & Blood brain barrier \\
\hline $\mathrm{C}$ & Centigrade \\
\hline cAMP & Cyclic adenosine monophosphate \\
\hline $\mathrm{CB}$ & Cannabinoid \\
\hline CB1 & Cannabinoid type 1 receptor \\
\hline CB2 & Cannabinoid type 2 receptor \\
\hline CBD & Cannabidiol \\
\hline $\mathrm{CCL}$ & C-C motif chemokine \\
\hline $\mathrm{c} / \mathrm{d}$ & Cycles per degree \\
\hline $\mathrm{CDC}$ & United States Centers for Disease Control and Prevention \\
\hline CD16/32 & Cluster of differentiation molecules 16 and 32 (M1 marker) \\
\hline CD206 & Cluster of differentiation molecule 206 (M2 marker) \\
\hline cGMP & Cyclic guanosine monophosphate \\
\hline CLSM & Confocal scanning microscopy \\
\hline CNS & Central nervous system \\
\hline CS & Contrast sensitivity \\
\hline CTE & Chronic traumatic encephalopathy \\
\hline
\end{tabular}




\begin{tabular}{|c|c|}
\hline CXCL & C-X-C motif chemokine \\
\hline DAI & Diffuse axonal injury \\
\hline DAMPS & Damage-associated molecular patterns \\
\hline DoD & United States Department of Defense \\
\hline DTI & Diffusion tensor imaging \\
\hline ERG & Electroretinogram \\
\hline EYFP & Enhanced yellow fluorescent protein \\
\hline $\mathrm{Fc}$ & Fragment crystallizable region of an antibody \\
\hline GBM & Glioblastoma multiforme \\
\hline GFAP & Glial fibrillary acidic protein \\
\hline Iba-1 & Ionized calcium-binding adapter molecule 1 \\
\hline IFN- $\gamma$ & Interferon gamma \\
\hline $\mathrm{IHC}$ & Immunohistochemistry \\
\hline IL & Interleukin \\
\hline INL & Inner nuclear layer of the retina \\
\hline ip & Intra-peritoneal injection \\
\hline IPL & Inner plexiform layer of the retina \\
\hline LCD & Liquid crystal display \\
\hline LE & Left eye \\
\hline LGN & Lateral geniculate nucleus \\
\hline LM & Light microscopy \\
\hline LOT & Left optic tract \\
\hline LPS & Lipopolysaccharide \\
\hline
\end{tabular}




\begin{tabular}{|c|c|}
\hline M0 & Resting/quiescent microglia \\
\hline M1 & Classically activated microglia \\
\hline M2 & Alternatively activated microglia \\
\hline MHC II & Major histocompatibility complex type II \\
\hline mRNA & Messenger ribonucleic acid \\
\hline mTBI & Mild traumatic brain injury \\
\hline $\mathrm{NADPH}$ & Nicotinamide adenine dinucleotide phosphate \\
\hline NeuN & Rbfox3, a neuronal biomarker \\
\hline NFL- & Nerve fiber layer of the retina \\
\hline NHS & Normal horse serum \\
\hline NMDA & N-methyl-D-aspartate receptor \\
\hline NO & Nitric oxide \\
\hline NOT & Nucleus of the optic tract \\
\hline OCT & Optical coherence tomography \\
\hline O.C.T. & Optimal cutting temperature (mounting medium) \\
\hline $\mathrm{OEF} / \mathrm{OIF}$ & Operation Enduring Freedom/Operation Iraqi Freedom \\
\hline $\mathrm{OKN}$ & Optokinetic nystagmus \\
\hline OKT & Optokinetic \\
\hline ON & Optic nerve \\
\hline ONL & Outer nuclear layer of the retina \\
\hline OPL & Outer plexiform layer of the retina \\
\hline OT & Optic tract \\
\hline OX6 & Also known as CD74, a marker of MHC II in mice \\
\hline
\end{tabular}




\begin{tabular}{ll} 
PB & Phosphate buffer \\
PBX & Phosphated buffer with Triton ${ }^{\mathrm{TM}} \mathrm{X}-100$ \\
pERG & Pattern electroretinogram \\
PKC- $\gamma$ & Protein kinase C gamma type \\
PLP & Paraformaldehyde-Lysine-Periodate \\
PPD & P-phenylenediamine \\
psi & Pounds per square inch \\
PTSD & Post-traumatic stress disorder \\
RE & Right eye \\
RGC & Retinal ganglion cell (layer) \\
ROS & Reactive oxygen species \\
ROT & Right optic tract \\
RPE & Retinal pigmented epithelium \\
rtPCR & Tumor necrosis factor alpha \\
SC & Transient receptor potential cation channel \\
SMI-32 & Transcription polymerase chain reaction \\
TBI & Traperior colliculus \\
TGF- $\beta$ & Non-phosphorylated neurofilament H 1 type cell \\
Th1 & Traumatic brain injury \\
TRF- $\alpha$ & Trike receptor 4 \\
\hline
\end{tabular}




$\begin{array}{ll}\text { VA } & \text { Visual acuity } \\ \text { VEP } & \text { Visual evoked potential } \\ \text { 0V } & \text { 0-psi (sham) blasted animals treated with vehicle } \\ \text { 2-AG } & \text { 2-arachidonylglycerol } \\ \text { 50SMM } & \text { 50-psi blasted animals treated with SMM-189 } \\ 50 \mathrm{~V} & \text { 50-psi blasted animals treated with vehicle } \\ 5-\mathrm{HT}_{1 \mathrm{~A}} & \text { 5-hydroxytryptamine receptor (serotonin) }\end{array}$




\section{CHAPTER 1. INTRODUCTION AND BACKGROUND}

\section{History and Epidemiology of TBI}

Traumatic brain injury (TBI) is the top cause of neurological dysfunction and death in most age groups worldwide ${ }^{1,2}$. In the United States alone, it has been estimated that as many as 3.8 million people per year suffer from some sort of traumatic brain injury ${ }^{3}$. Recent headlines have brought TBI and its mildest form, the concussion, to the attention of people around the country, with professional football players and physicians alike labeling the concussion as a new epidemic plaguing America ${ }^{4}$. Despite this being thought of as a modern phenomenon, it is a malady that has afflicted humans since the beginning of recorded history. One of the oldest medical texts ever discovered, an Egyptian papyrus dating back to the 16th century BC, includes complex descriptions of traumatic head wounds, both penetrating and non-penetrating, succinctly describing symptoms and outlining rudimentary treatments used by high priests, the medical doctor equivalent of the time ${ }^{5,6}$. Indeed, injuries to the head would have been a major concern to early humans, as they were well understood to be some of the most resistant to tribal medicine and a cause of life-long disability. Classically, TBI was probably encountered as a result of contusive, penetrating, or acceleration-deceleration (A-D) injury. In the modern day, most instances of TBI occur as a result of falls, motor vehicle accidents, and assaults ${ }^{7}$, with males in general and populations in the military, professional sports, and construction work being at the highest risk ${ }^{8,9}$. Those most likely to be affected by TBI are some of the most vulnerable patient populations in our society, being the very young (0-4 years of age) and the very old (above 75 years of age) ${ }^{3}$. Thus, the focus of research aimed at prevention and treatment of TBI rightly continues to be a high priority nationally.

TBI categorization is yet to be standardized, and as such has somewhat broad definitions compared to many other diseases ${ }^{10,11}$. At its most general, it can be understood as any injury resulting from biomechanical disturbance of the brain and surrounding tissue. The United States Department of Defense (DoD) has implemented a categorization scheme for TBI that is widely used in both research and clinic settings, and will be adopted for use in the remainder of this dissertation ${ }^{12}$. According to the DoD, TBI can be classified as either mild, moderate, or severe based on a number of criteria that serve as a readout of neurocognitive function ${ }^{13}$. See Table 1-1 for a complete explanation of the DoD's TBI severity criteria. Importantly, mild TBI (mTBI) is unique from the other more severe forms in that it has structurally normal radiologic findings, a normal to near-normal score on the Glasgow coma scale, and does not necessarily involve a loss of consciousness or altered mental status ${ }^{13}$. The apparent lack of physical findings are in stark contrast to the myriad of symptoms patients experience after mTBI, many of which can be debilitating. Mild TBI is thought to comprise anywhere from 80$95 \%$ of all TBI seen in hospitals ${ }^{9}$, and has been estimated by the CDC to account for over 16 billion dollars in US healthcare spending over the lifetime of affected individuals

${ }^{11}$. With a prevalence of over 500 per 100,000 people in the population per year ${ }^{14,15}$, it is 
Table 1-1. Department of Defense traumatic brain injury classification criterion.

\begin{tabular}{llll}
\hline Criteria & Mild & Moderate & Severe \\
\hline Structural imaging & Normal & $\begin{array}{l}\text { Normal or } \\
\text { abnormal }\end{array}$ & $\begin{array}{l}\text { Normal or } \\
\text { abnormal }\end{array}$ \\
Loss of consciousness & $0-30 \mathrm{~min}$ & $\begin{array}{l}>30 \mathrm{~min} \text { and } \\
<24 \mathrm{hrs}\end{array}$ & $>24 \mathrm{hrs}$ \\
$\begin{array}{l}\text { Alteration of consciousness/ } \\
\text { mental state* }\end{array}$ & $\begin{array}{l}\text { A moment } \\
\text { up to } 24 \mathrm{hrs}\end{array}$ & $\begin{array}{l}>24 \text { hours. Severity based on } \\
\text { other criteria }\end{array}$ & \\
$\begin{array}{l}\text { Post-traumatic amnesia } \\
\text { O-1 day }\end{array}$ & $\begin{array}{l}>1 \text { and }<7 \\
\text { days }\end{array}$ & $>7$ days \\
$\begin{array}{l}\text { Glascow Coma Scale (best } \\
\text { available score in the first } 24\end{array}$ & $13-15$ & $9-12$ & $<9$ \\
hours) & & &
\end{tabular}

*Alteration of mental status must be immediately related to the trauma to the head. Typical symptoms would be: looking and feeling dazed and uncertain of what is happening, confusion, difficulty thinking clearly or responding appropriately to mental status questions, and being unable to describe events immediately before or after the trauma event.

Reprinted with permission. Defense, D. O. \& Affairs, D. O. V. Management of Concussion/mild Traumatic Brain Injury. 1-112 (2009). 
estimated that over 5 million people in the US are currently living with mTBI-related disabilities ${ }^{16}$.

Milder cases of TBI, usually associated with sports, are known colloquially as concussions and are often brushed off without a doctor or emergency department visit ${ }^{17}$. This is probably at least partially due to pressures associated with return to play or other social stigmas ${ }^{18}$, but also from a general public misconception that concussions have very few long term sequelae ${ }^{19}$. In the early 2000 's, when professional football players began to manifest symptoms similar to dementia and other neuropsychiatric conditions at increasingly younger ages, attention became focused on the role of concussive, and even sub-concussive injuries on the long-term health of athletes in football and other highimpact sports ${ }^{20}$. Hyper-phosphorylated tau accumulation indicative of neural microtubule disruption was found in the brains of even very young, non-professional athletes, head-bangers, and wrestlers, many with no prior history of concussion ${ }^{21}$. This disease became known as Chronic Traumatic Encephalopathy, and is probably related to a precursor found exclusively in boxers called Dementia Pugilistica ${ }^{22}$. It is now widely recognized in the medical world that concussion is the mildest of a spectrum of injuries involving biomechanical disruption of the soft tissue of the brain, with manifestations from very minor to very severe in both clinical outcome and effect on patient quality of life ${ }^{22}$.

With the advent of modern warfare, a new mechanism of brain injury was invented, one caused by exposure to extremely high pressure gradients, known as blast waves, that are emitted from explosive detonations. As early as World War I, the disease called "shell shock" was known to be associated with blast exposure, and was described as being characterized by specific neurological and psychological sequelae ${ }^{23}$. In the medical community, it was widely debated as to whether the disease had any structural basis, and veterans did not begin receiving benefits for shell shock until World War II ${ }^{24}$. The long term psychopathology many of these individuals suffered with after returning from combat eventually morphed into what we now call post traumatic stress disorder (PTSD) ${ }^{25}$. More modern wars brought a new breed of solider, the so-called "breacher," the men responsible for improvised explosive device recovery and controlled detonation. In doing this, they are exposed to hundreds or thousands of blasts across their military career. It was not immediately understood why, but these individuals began to suffer with numerous debilitating side effects as a result of this blast exposure ${ }^{26}$. Eventually it became recognized that even in the absence of blood or outward signs of trauma, these blast exposures were somehow causing damage to an individual's brain. Today, overpressure blast-induced TBI, or bTBI, is considered to be a subset of mild traumatic brain injury, and still has close associations with PTSD, as well as long term motor, auditory, and visual deficits. Blast TBI will be discussed at length later in this dissertation. 


\section{Consequences of TBI}

The physical effects of mTBI can be quite varied, depending both on the injury severity and localization, but can often have a profound impact on the patient's quality of life ${ }^{27}$. Clinical manifestations of mTBI are frequently referred to as post-concussion syndrome, although this is somewhat of a misnomer since the symptoms are also seen in more severe brain injury as well ${ }^{28}$. They can include a variety of behavioral, neurocognitive, and somatosensory symptoms that are traditionally thought of as being transient, lasting from weeks to as long as 3 months after the injury ${ }^{2,29}$. However, as many as $15 \%$ of people with mTBI still experience these symptoms long after the 3 month time point ${ }^{30}$, suggesting a tangible neurobiological etiology. See Table 1-2 for a comprehensive summary of TBI-associated symptoms. The presence of acute-onset postconcussive symptoms is correlated with worse neurocognitive function as long as a year after the injury, and as such indicate the presence of some underlying neurobiological basis that could have potentially permanent effects ${ }^{30}$. Accordingly, studies using diffusion tensor imaging (DTI) have found white matter abnormalities that significantly correlate with post-concussion symptom severity, even more so than cognitive function tests alone ${ }^{31}$. This highlights the importance of seemingly subjective readouts, such as quality of life and patient-reported symptoms, in determining the extent of neuropathology after mild brain injury.

Somatic symptoms associated with TBI can include headaches, dizziness, nausea, and fatigue ${ }^{16,29}$ Of these symptoms, headache is the most common ${ }^{32}$, and seems to be more closely related to mild brain injury - one study found the daily headache incidence in the mildest category of TBI to be nearly $80 \%{ }^{33}$. Another common, yet poorly understood symptom of mTBI is chronic fatigue. Research shows that despite it being more common in women, it is not directly related to other comorbid conditions, suggesting a neurobiological etiology relating directly with the brain injury ${ }^{34}$. The mechanism behind this symptom has yet to be elucidated, although newer research shows a potential link with post-TBI neuroendocrine dysfunction and hypopituitarism ${ }^{35,36}$. Dizziness and nausea are most commonly thought to be related to vestibular dysfunction ${ }^{37}$, and are not well researched in the literature.

Other symptoms associated with mTBI involve problems with memory, concentration, focus, and attention ${ }^{38}$. These are the most widely and extensively researched of the post-TBI sequelae and are closely linked with white matter damage, or diffuse axonal injury (DAI) ${ }^{39,40}$. Diffuse axonal injury is an almost universal finding in even mild TBI ${ }^{41}$, and acute DAI findings on MRI correlate highly with long-term cognitive function performance ${ }^{42}$. The non-focal nature of DAI likely accounts for the lack of specificity of many of the cognitive symptoms associated with mTBI ${ }^{40}$.

\section{Neuropsychiatric symptoms in mTBI}

The link of psychiatric illness with brain injury is a complicated one, since preinjury factors (genetic, environment, behavioral), as well as post-injury factors 
Table 1-2. Health effects associated with TBI.

\begin{tabular}{ll}
\hline Category & Description \\
\hline Cognitive & $\begin{array}{l}\text { Deficits in: attention; learning and memory; executive functions } \\
\text { like planning and decision-making; language and communication; } \\
\text { reaction time; reasoning and judgment }\end{array}$ \\
$\begin{array}{ll}\text { Behavioral/ } \\
\text { Emotional }\end{array}$ & $\begin{array}{l}\text { Delusions; hallucinations; severe mood disturbance; sustained } \\
\text { irrational behavior; agitation; aggression; confusion; impulsivity; } \\
\text { social inappropriateness }\end{array}$ \\
Motor & $\begin{array}{l}\text { Changes in muscle tone; paralysis; impaired coordination; changes } \\
\text { in balance, or trouble walking }\end{array}$ \\
Sensory & Changes in vision and hearing; sensitivity to light \\
Somatic signs & Headache; fatigue; sleep disturbance; dizziness; chronic pain \\
and symptoms &
\end{tabular}

This table gives a comprehensive overview of the symptoms associated with TBI.

Reprinted with permission. Centers for Disease Control and Prevention (CDC). Report to Congress on Mild Traumatic Brain Injury in the United States: 1-56 (Centers for Disease Control and Prevention, 2003). at

$<$ http://www.cdc.gov/traumaticbraininjury/pdf/mtbireport-a.pdf $>$ 
(psychosocial) and injury severity/localization, likely play a critical role in long-term neuropsychological outcome after $\mathrm{mTBI}^{43}$. As a result, the role of $\mathrm{mTBI}$ in neuropsychiatric illness has been somewhat controversial in the literature. One strongly worded review written in 2005 pointed to the numerous conflicting studies done on a number of neuropsychological end points and concluded that there was no strong evidence that mTBI had any long-term effects on behavior ${ }^{44}$. Despite this, a large body of literature exists that points to associations of mTBI with numerous neuropsychiatric sequelae, including depression, impulse control, anxiety, suicidal thoughts, amnesia, aggression, apathy, and impulsivity, among others ${ }^{28}$. One review found the incidence of depression to be the highest of these symptoms, occurring in anywhere from $15-61 \%$ of individuals after mTBI, even when controlling for those with episodes of depression prior to injury ${ }^{45}$. Additionally, even in the absence of depression symptoms acutely postinjury, the risk for developing clinical depression is still elevated for many decades after $\mathrm{TBI}^{43}$. Studies have found significant deficits in cognitive control in individuals with a history of mTBI, which increase with increasing number of mTBI incidents ${ }^{46}$. Importantly, these deficits are present in the absence of any clinical signs or symptoms, hinting at a dysfunction in the brain's impulse control circuitry in individuals who by all account are clinically normal. Finally, the most robust and well-corroborated evidence shows a link between mTBI and PTSD ${ }^{47}$, which is said to occur in anywhere from 17 to $30 \%$ of individuals after $\mathrm{mTBI}^{48}$, and has significant correlations with self-reported postconcussive symptom severity ${ }^{49}$. However, the literature also shows the difficulty in disentangling the role of neurobiological and psychological causes in cases of PTSD and concurrent mTBI, since many instances of mTBI occur in times of intense psychological distress ${ }^{48}$.

\section{Mild TBI and vision}

General. One of the most common complaints associated with mTBI comes in the form of vision problems ${ }^{13}$, with as many as $75 \%$ of patients reporting some sort of visual disturbance due to their injury ${ }^{50}$. These visual symptoms often exist in the absence of any outward damage to the globe of the eye ${ }^{51}$, and as such indicate a complicated underlying neuro-ophthalmologic pathology. With as much as $50 \%$ of the brain's mass dedicated either directly or indirectly to visual processing ${ }^{52}$, it stands to reason that stretching and sheering of axons diffusely throughout the brain, as occurs in mTBI, could impact the visual system in a myriad of ways ${ }^{53}$. Indeed, visual performance is such a reliable readout of even mild forms of brain injury, that it is routinely used in sideline tests for determining return to play in high school and college sports after a head injury ${ }^{54-56}$ and to longitudinally monitor recovery from mTBI ${ }^{57,58}$. The diffuse nature of the mTBI injury likely accounts for the non-specific and often difficult-to-pin-down nature of mTBI-associated vision complaints as reported by patients, many of whom experience significant difficulties with the activity of daily life, yet have normal or nearnormal visual acuity findings on physical examination ${ }^{51}$.

Specific visual disturbances. TBI-associated vision symptoms can vary depending on injury severity and range from headaches, photophobia, blurred/double 
vision, and difficulty reading to partial or complete blindness ${ }^{59,60}$. Many of these vision symptoms are thought to be primarily related to dysfunction in the oculomotor system, with as many as $90 \%$ of individuals with acquired brain injury manifesting some sort of oculomotor problem ${ }^{61}$. Oculomotor disturbance can manifest as vergence insufficiency, difficulties with smooth pursuit, and saccadic dysfunction, all leading to significant difficulties with activities of daily life ${ }^{62,63}$

According to Kapoor and Cuiffreda, vision problems related with mTBI can be categorized into five main areas of dysfunction: accommodation, version, vergence, visual field integrity, and photosensitivity ${ }^{64}$. Each of these areas will be briefly discussed, with emphasis on how these clinical signs translate into functional problems (symptoms) for patients, and their possible pathophysiology, when known.

Accommodation. Accommodation is the ability of the eye to change focus when moving from near-to-far, or far-to-near vision by altering the shape of the lens ${ }^{65}$. Neural implementation of accommodation is a complex system, but is primarily driven by a reflex arc through the lateral geniculate nucleus (LGN), visual cortex, cerebellum, midbrain, and cranial nerve III ${ }^{66}$. In brain injured patients, this reflex arc can be slowed (accommodation infacility), decreased in strength (accommodation insufficiency), or hyperactive (accommodation spasm) ${ }^{67}$, all of which manifest as either constant or intermittent bouts of blurred vision ${ }^{64}$. Occasionally, these deficiencies can be ameliorated by use of corrective lenses, but not always ${ }^{68}$.

Vergence. Vergence is the ability of both eyes to move toward or away from one another in order to retain binocular vision of stationary and moving objects within the visual field ${ }^{69}$. It, like accommodation, involves a complex reflex arc through the LGN, visual cortex, cerebellum, midbrain, and numerous cranial nerves. Vergence problems most commonly manifest as deficiencies in horizontal convergence (convergence insufficiency), and can result in diploplia (double vision), difficulty reading, headaches, nausea, and general difficulty with near tasks ${ }^{70,71}$. These are the most common, and arguably most debilitating of the symptoms experienced by mTBI sufferers with visual dysfunction ${ }^{62}$.

Version. Version refers to coordinated conjugate eye movements, such as saccades, which allow binocular vision of stationary and moving objects. Saccades are reflexive eye movements that occur very rapidly, at 200-700 degrees per second, and are important for visual inspection and vision integration ${ }^{72}$. In brain injured patients, version dysfunction manifests primarily as saccadic dysfunction, abnormal smooth pursuit, and fixation difficulties ${ }^{64}$. While version and vergence circuits likely overlap to a significant extent, studies have found that focalized injuries can result in selective sparing of one over the other ${ }^{73}$. Accordingly, the version circuit has been found to involve additional areas of the brain, such as the superior colliculus, cingulate cortex, and regions of the prefrontal cortex ${ }^{74}$. Importantly, many of the circuits controlling saccade generation are also important in directing visual attention ${ }^{75}$, another known area of dysfunction in mTBI 76. Overall, the main symptoms associated with version dysfunction manifest as 
difficulties with reading such as skipping words or lines, having to re-read words, and an overall decrease in reading speed and efficiency ${ }^{64}$.

Visual field defects. Visual field deficits seen in TBI are more well-characterized than the other symptoms of TBI-associated visual dysfunction, and can represent direct damage to either the proximal or distal retino-geniculo-calcarine pathway ${ }^{65}$. Direct injury to the optic nerve and chiasm are possible findings with partial or complete blindness ${ }^{77}$, and the nature of the visual field defect can often help localize the site of injury. Symptoms of partial visual field defects often manifest as perceptual or spatial deficits, for example not noticing one side of a room or words on one side of a page. These are generally associated with more severe injuries, and are rare in mild TBI ${ }^{77}$. Other changes in visual character, such as waviness or graininess, are not well understood and may be linked with damage to the visual association areas ${ }^{64}$.

Photoallodynia. Finally, the last category of injury described by Kapoor and Cuiffreda is the painful aversion to light, photoallodynia, or abnormal light sensitivity, photophobia $^{51}$. Photosensitivity has been the subject of much research, since it is a symptom commonly associated with a number of other diseases ${ }^{78}$. Light sensitivity is known to be predominantly, but not exclusively, mediated through intrinsically photosensitive retinal ganglion cells within the eye ${ }^{79}$, and as such may occur in many direct ocular injuries. However, photosensitivity is also a common symptom in migraine headaches. This suggests other extraocular mediators can give rise to photophobia. Recent research indicates the trigeminal nerve, and the cortico-trigeminal pathway likely play an important role in neuropathic causes of photosensitivity ${ }^{80,81}$. Whether the presence of light sensitivity after mTBI is mediated by ophthalmic or neurologic pathways is not currently known, and likely depends on the nature and severity of the injury.

The purpose of this dissertation is to focus on the visual manifestations of mTBI. Further discussion of this will follow description of important aspects of TBI pathophysiology that require a general overview. We will then return to the discussion of the visual system in the section on treatments for TBI.

\section{TBI Pathophysiology}

An important hallmark of mild traumatic brain injury is its apparent lack of consistent neurophysiological findings when compared with other forms of brain pathology, making it an elusive and somewhat controversial disease to study, until recent events brought it to mainstream attention ${ }^{82}$. Nonetheless, focused and persistent neuroanatomical and neuropathologic studies have elucidated many of the underlying mechanisms to this disease. For categorical purposes, the field of TBI research has identified two phases of the TBI pathophysiology, roughly relating to acute, inevitable parenchymal damage, and long-term, possibly preventable damage. The acute effects of the biomechanical insult on the brain are commonly referred to as primary injury, and represent the first wave of actions that can result in long-term consequences ${ }^{83}$. 
Subsequent immune, cerebrovascular, and metabolic responses to the brain injury are called the secondary injury, and are the more feasible targets for potential therapies ${ }^{83}$. These will be discussed in turn.

\section{Primary injury}

The pathophysiology of brain injury begins with a biomechanical disturbance, which can stretch and shear axons and cell bodies within the soft tissue of the brain. White matter tracts are the neuronal superhighways of the brain, consisting of tightly organized and directionally arranged axon bundles that transmit electrical signals from one region of the brain to another. Because of their highly ordered nature, white matter tracts are uniquely susceptible to stretch and torsion during head acceleration and trauma, even more so than regions of tightly-packed neuronal cell bodies such as grey matter ${ }^{84}$. As a result, even mild forms of brain injury can result in widespread, diffuse axonal damage known as DAI ${ }^{85,86}$. Within individual axons, microtubules act as the structural framework to shuttle waste products, organelles, and newly synthesized protein to and from the soma. During brain trauma, microtubules can shear and break, disrupting the transport processes that are essential to cell survival ${ }^{87,88}$. Transport products are unable to be shuttled to their normal locations in the cell, and instead build up, forming localized areas of axon swelling (axon bulbs), which are clearly visible on histological analysis ${ }^{89}$. Studies have found that forces exerted on the head are directionally important as they relate to axonal injury ${ }^{90}$. Rotational head accelerations tend to be more important for axonal shear damage than simple linear acceleration ${ }^{91}$.

During brain trauma, head movement or brain deformation can result in mechanical disturbance of cellular membranes ${ }^{92}$. Studies have shown that there is widespread blood brain barrier (BBB) compromise ${ }^{93}$, and cellular membrane micropore formation, allowing uncontrolled leakage of ions into the extracellular space ${ }^{94}$. This mechanical disturbance also releases inflammatory cytokines and chemokines into the extracellular milieu and exposes damage associated molecular patterns (DAMPS), both of which induce an acute inflammatory response by resident immune cells in the brain ${ }^{95}$. Additionally and concurrently, mechanical disruption causes the acute release of the excitatory neurotransmitter glutamate from intact cells, which induces indiscriminate neuronal depolarization ${ }^{96}$. Glucose metabolism in the brain is increased rapidly in response to injury as ATP-powered ion channels work to equilibrate this sudden ion flux, quickly depleting glucose stores ${ }^{97}$. This results in aerobic metabolism and the buildup of lactic acid, thought to be responsible for concomitant cerebral edema, while widespread NMDA receptor activation floods cells with $\mathrm{Ca}^{2+}$, resulting in mitochondrial dysfunction and a decrease in cytochrome oxidase expression ${ }^{98}$.

\section{Secondary injury}

In addition to the significant metabolic and mechanical disruption after trauma, immune activation likely plays an important role in the sustained pathology of TBI ${ }^{99}$. 
Monocyte-derived microglia are the resident immune effector cells within the CNS. In their resting state, they constantly sample the extracellular milieu and respond to signals within the surrounding microenvironment. Damage during neurotrauma and subsequent exposure of specific cellular proteins which are not normally present in the microenvironment (known as DAMPS) induce activation of toll-like receptor 4 (TLR4) found on microglia ${ }^{100}$, inducing reactive polarization to an M1 phenotype ${ }^{101}$. M1 reactive microglia release the inflammatory cytokines IL-1, IL-6, and TNF- $\alpha{ }^{102}$, which can induce peripheral immune cell extravasation, in addition to chemotactic signals such as CCL2 that attract other resident microglia to the area. They also act as antigen presenting cells, phagocytosing cellular breakdown products and processing them for expression to peripherally-derived immune cells, or traversing the body themselves to present these proteins to dendritic cells in regional lymph nodes ${ }^{103}$. This begins the activation of the adaptive immune response, and $\mathrm{B}$ cells begin producing antibodies to target these self-derived proteins ${ }^{99}$. In this way, the acute immune response gives way to a long-term, sustained immune response targeted at self-proteins erroneously identified as being a threat. T-cell mediated destruction of myelin and other structural components may give rise to long-lasting cell death and immune over-activation in cases of subsequent trauma, in a phenomenon referred to as trauma-induced autoimmunity ${ }^{104}$.

\section{The Role of the Immune System in TBI Pathology}

\section{The role of peripheral cells}

Under normal conditions, the expression of pro-inflammatory cytokines, chemokines, and complement are low in the healthy brain ${ }^{105}$. However, following brain trauma their expression rises precipitously ${ }^{106,107}$. Virtually all cell types within the CNS are capable of expressing pro-inflammatory cytokines in response to trauma ${ }^{108}$, and as such even diffuse injury as is seen in mTBI can cause widespread activation of the immune system. Under physiologic conditions, the BBB keeps the CNS an immune privileged region ${ }^{109}$, but during brain trauma, this barrier becomes compromised and leaky ${ }^{93}$, allowing immune cell adhesion and infiltration from the periphery into the CNS ${ }^{110}$, driven by inflammatory chemotactic signals. Studies show that circulating monocytes migrate to regions of BBB compromise, extravasate, and differentiate into antigen presenting dendritic cells or macrophages in response to trauma ${ }^{111}$. Additionally, tissue-specific dendritic cells probably also reside in the CNS ${ }^{103}$. The fate of either type of dendritic cell is the same- both phagocytose proteins from the region of trauma and navigate to regional lymph nodes to present these antigens to $T$ lymphocytes, which can then go on to activate the adaptive immune response ${ }^{112}$. Activation of self-specific, antibody-producing $\mathrm{B}$ cells, and initiation of cytotoxic $\mathrm{T}$ cells are both hypothesized mechanisms for continued damage after $\mathrm{TBI}^{113}$. 


\section{Microglia}

Although dendritic cells play an important role, the predominant cell type that mediates the immune response in the CNS after trauma is the resident microglia. More than just tissue-specific macrophages, microglia are also glial cells, which perform important support functions and release neurotropic factors under physiologic conditions 114. Critically, they also respond to injury, infection, and trauma, acting as myeloidderived resident tissue monocytes. They are integral in debris and necrotic tissue removal, while also helping maintain tissue integrity after injury ${ }^{115}$. These two seemingly disparate roles speak to the ability of microglia to alter their polarization, or phenotypic and gene expression profiles, adapting to environmental signals and temporal progression of injury under pathologic conditions. Microglial polarization states are identified by their distinct cytokine expression in response to chemical stimuli ${ }^{116}$, as well as their unique cell surface receptor makeup ${ }^{117}$.

Polarization. While microglia are in their resting, or M0 state, they display a highly arborized "ramified" morphology that assists in their constant monitoring of the extracellular environment ${ }^{118,119}$. Upon activation, their phenotypes can be roughly categorized into two major polarization states: either M1 or M2. M1 is the proinflammatory, or neurotoxic state, whereas M2 is the anti-inflammatory or neuroprotective state ${ }^{120}$. Although overly simplified, the imposed dichotomy of M1 versus M2 is useful when discussing the role microglia play in trauma and subsequent immune activation, similar to the nomenclature of Th1 and Th2 lymphocytes ${ }^{116}$, and as such is widely used in the literature.

The M1 polarization state is neurotoxic ${ }^{121}$ and known to be detrimental to tissue repair after injury ${ }^{122}$. Indeed, the number of M1 microglia present is highly correlated with white matter injury in TBI ${ }^{123}$, and plays a prominent role in a number of other neuropathologies ${ }^{124}$. In brain trauma, microglia become activated in a graded manner to the M1 phenotype by way of TLR4 activation ${ }^{100}$. In this activation state, they retract their processes and morph into amoeboid phagocytic cells, expressing a full gamut of MHC and cellular adhesion molecules to assist in immune functions ${ }^{101}$. They also release the inflammatory cytokines IL-1, IL-6, and TNF- $\alpha^{102}$, which can act to further aggravate the injury. Additionally, chemotactic cytokines CCL2, CXCL9, and CXCL10 are released, which play an important role in leukocyte recruitment to sites of injury 124,125. An unintended consequence of M1 activity is the release of additional substances that can be directly neurotoxic, such as nitric oxide, glutamate, and reactive oxygen species created by unchecked NADPH oxidase activity ${ }^{120,126}$.

An alternative and equally important fate of microglia in response to injury is a concurrent or sometimes late-phase predominant polarization to an M2 phenotype, in which anti-inflammatory cytokines such as IL-4 and TGF- $\beta$ are released instead ${ }^{127}$. M2 polarized microglia are known to be neuroprotective in many CNS pathologies ${ }^{128}$, and their presence correlates with better prognosis after spinal cord trauma ${ }^{121}$, as well as the initiation of re-myelination after traumatic axonal injury ${ }^{129}$. The ability of extracellular 
signals to alter microglial polarization and thus disease progression is clearly fertile ground for potential therapies, and will be addressed at length later.

Timeline. The timeline of microglial activation and polarization is somewhat disputed in the literature, since both M1 and M2 microglia likely exist at all times after traumatic insult ${ }^{120}$. However, most studies agree that microglial activation begins within hours of injury, peaking within a week, and then tapering off at two weeks ${ }^{123}$.

Microglial polarization is driven by signals released from cells and other events in the immediate microenvironment, and is thought to be graded based upon the concentration of inflammatory signaling molecules they are exposed to ${ }^{130}$. M1 polarization is induced by exposure to IFN- $\gamma$, TNF- $\alpha$, and LPS ${ }^{131}$, while M2 polarization is driven primarily by IL-3, IL-4 , and IL-10 ${ }^{131,132}$. Some studies show a sub-acute preponderance of M2 microglia within the first few days of injury ${ }^{127}$, with a quick induction of M1 polarization soon after, as injured neurons begin releasing pro-inflammatory signaling molecules such as ATP and glutamate ${ }^{126,133}$. Numbers of highly reactive microglia begin to drop off at the two week time point, but low levels of reactive microglia can exist for sometimes months to years after an acute injury ${ }^{120,134}$.

\section{Animal Models Used in TBI Research}

A number of different animal models have been developed to both investigate the underlying pathology of TBI, and test potential therapies for translation to human populations. In order to understand the model used for the purposes of this dissertation, and how it fits into the larger picture of the TBI research field, a brief overview of other animal models of acquired brain injury is warranted.

Roughly five main approaches for creating acquired brain injury in animal models exist and are in current use: Static crush, Overpressure blast, Non-impact head acceleration, Non-penetrating impact-driven head acceleration, and Penetrating impactdriven head acceleration ${ }^{135}$. To better understand these and how they relate to one another, please refer to an excellent graphic borrowed from Cernak's comprehensive review of the subject (Figure 1-1). Briefly, Cernak has divided injuries into two broad categories: dynamic- where the acceleration, velocity, amplitude, and duration are variable; and static- where acceleration and velocity are not applicable, because the injury consists of only a known force amplitude for a fixed period of time. Only one model fits into the static category, that of crush, and is used predominantly to study secondary injury due to the highly artificial nature of the primary injury ${ }^{135}$. For understandable reasons, static crush models are not able to replicate the complicated brain deformation seen in most TBI injuries, and for this reason we will not delve into them further. It is important to note, however, that crush injuries are common in investigation of traumatic optic nerve axon pathology, and as such have a place of importance in the field of vision research 136,137. The second, dynamic category is further subdivided into either direct or indirect methods, which will now be discussed in greater detail. 


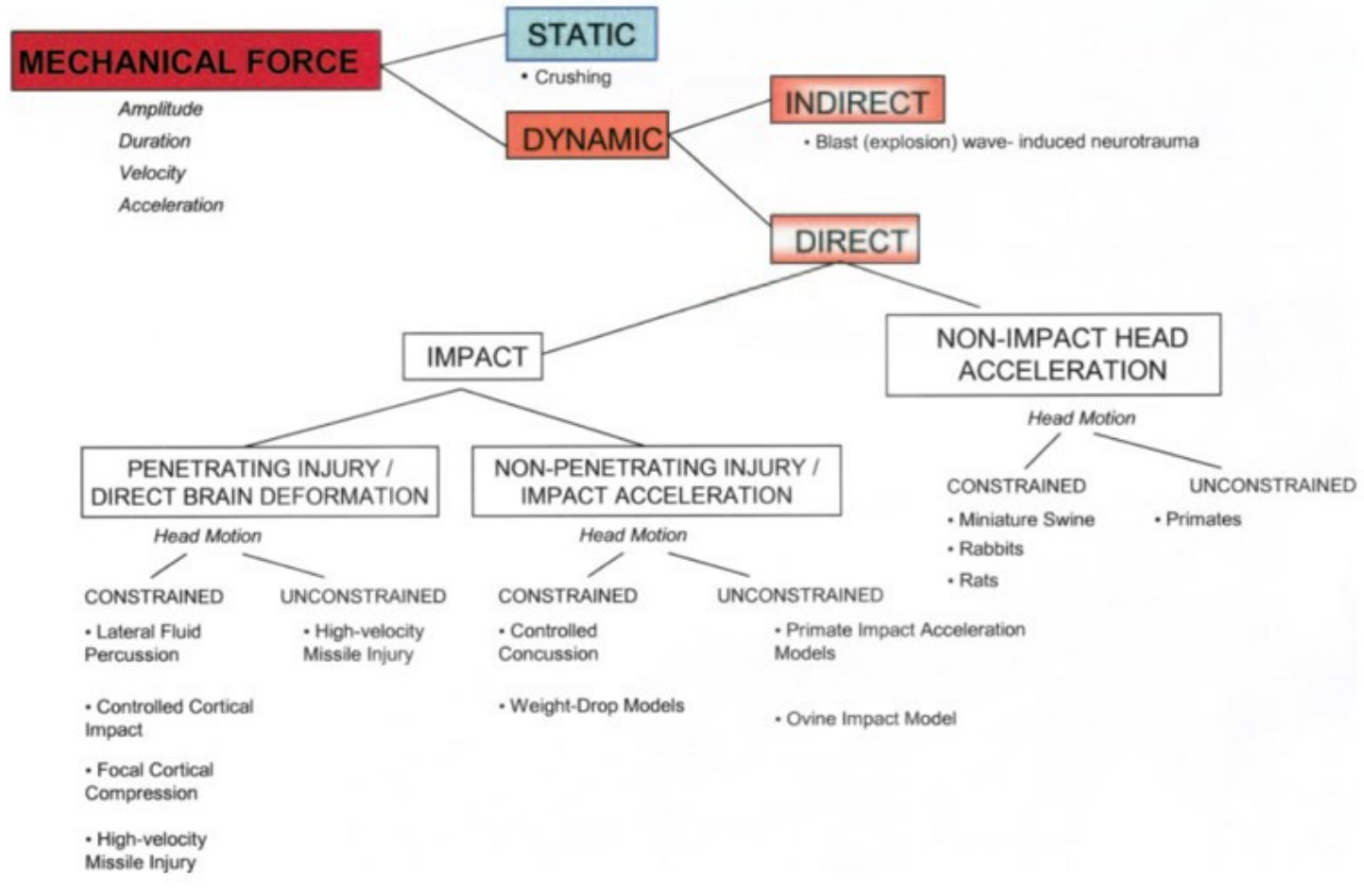

Figure 1-1. In vivo models of traumatic brain injury.

This figure shows the main categories of animal models used to study TBI. The model used in this dissertation work falls under the dynamic, indirect category.

Reprinted with permission. Cernak, I. Animal models of head trauma. Neurotherapeutics 2, 410-422 (2005). 


\section{Direct methods}

Direct methods of TBI use direct application of a force to the brain, in either an open-skull or closed-skull application. Many open-skull models require surgical removal of skin, muscle, and bone to expose the animal's dura mater and underlying brain tissue for injury. A fluid pressure pulse, or sometimes pressure-powered piston, is then used to inflict a controlled, replicable force to the brain. While these models are desirable for the highly replicable nature of the injury they produce ${ }^{138}$, the extensive surgery required makes it nearly impossible to study secondary effects of brain injury, since the significant inflammation caused by the open-skull procedure must invariably complicate the injury caused by the primary brain damage. At least one study has documented a replicable effect on the visual system using a direct fluid percussion model of TBI ${ }^{139}$. Other openskull models use penetrating mechanisms, in the form of missile or stab wounds, to inflict brain injury ${ }^{140}$. These result in a more severe TBI that is highly related to real world penetrating injuries, such as a gun shot, but are not able to replicate less severe, mild to moderate forms of closed-skull injury, and as such are inappropriate for research on mTBI or concussion.

Closed-skull direct methods include controlled concussion, impact-driven head acceleration, and non-impact head acceleration models ${ }^{141,142}$. Generally, these models are excellent replicas of real world injury, yielding diffuse axonal injury in regions seen in human cases of mTBI and concussion ${ }^{143}$. However, only after repeated mTBI have any of these models given definitive readouts of visual injury similar to that which is so prevalent in human cases of $\mathrm{mTBI}^{144}$, and are thus not helpful for investigating TBIassociated vision dysfunction after only a single incident.

\section{Indirect methods}

Indirect methods use large traveling pressure gradients known as blast waves as the mechanism of injury; they are done with a closed skull, and can be directed at either a focalized region of the skull, or the whole body. These are primarily used to replicate injuries seen in individuals who have been exposed to explosive detonations, such as military personnel. When an explosive detonates, it creates an ultra-high velocity pressure differential that travels in the form of a shock wave through the air radially. Upon encountering a fixed object, the energy from the shockwave is transferred in the form of kinetic energy, heat, and shear stress. The pressure wave interaction with the skull and brain is considered the primary effect of the blast wave ${ }^{145}$, and causes a rippling deformative force on the elastic tissue of the brain ${ }^{146}$ that can result in diffuse axonal injury ${ }^{147}$, axonal degeneration, and possibly eventual neuronal degeneration. The tissue distention can also lead to an increase in intracranial pressure, which may give rise to further neuroinflammation and neurodegeneration ${ }^{148}$. Secondary injury mechanisms then proceed as previously described with non-blast forms of TBI. Much evidence exists to support the idea that blast-associated mTBI is a unique form of injury ${ }^{149}$, which is confirmed by the high association of blast injury with particular neurological sequelae which are not as highly correlated with other forms of acquired brain injury ${ }^{150}$. 
However, some studies purport that, while being a unique injury mechanism, blast-related mTBI is probably not a unique pathology ${ }^{151}$. At face value, this seems reasonable at least as it applies to secondary injuries. To date, indirect blast overpressure models have been the most successful in replicating the specific effect on the visual system that is seen in human cases of mTBI ${ }^{152-155}$. For this reason, they represent the ideal model for investigating potential treatments for mTBI-associated visual dysfunction.

\section{Treatments for TBI}

\section{General}

Given the significant number of individuals affected by TBI every year, it would be assumed that much time and research money should have paved the way for many novel therapies to treat TBI and its associated sequelae. In fact, very few promising interventions or current treatments exist for both TBI in general, and TBI-associated visual symptoms more specifically. Overall, symptoms are treated on a case-by-case basis by specialists within a related field. Psycho-emotional symptoms are generally treated by a psychiatrist or psychotherapist, vision symptoms are treated by an optometrist, and neurological symptoms are treated by a neurologist, etc. These multidisciplinary intervention schemes have been relatively successful in improving patients' quality of life ${ }^{156}$, but also involve a significant monetary and time burden on patients who choose these methods of rehabilitation. Historically, and even up until the present day, physical and cognitive rest have been considered the cornerstone of concussion treatment and other mild forms of $\mathrm{TBI}^{157}$. This advice, however, seems to contradict much evidence that physical inactivity can actually exacerbate many disease conditions ${ }^{158}$, and seems based more in tradition than evidence. One review on the subject concluded that rest lasting more than three days after the injury probably yields no benefits on long term outcome ${ }^{158}$, while other studies have actually provided evidence for the benefit of a controlled physical exercise regime after mTBI, as long as other activities that could yield a second mTBI are avoided ${ }^{159}$.

Pharmacologic interventions for mTBI consist primarily of therapies targeted at alleviating symptoms, rather than the underlying pathology, but a few more broadly acting drugs have been investigated. Some have shown promise in animal models, others have made it all the way through to a full clinical trial, but none have shown efficacy at the control clinical trial phase. Included in these are steroids, progesterone ${ }^{160}$, and ketamine $^{161}$, to name a few. A recently published comprehensive review summarized the bleak landscape of TBI pharmaco-interventions by simply concluding that no single pharmaceutical intervention is proven to be efficacious for treatment of TBI and its associated symptoms ${ }^{162}$.

Other therapies proposed for TBI are less conventional. One such therapy, used primarily for treating more severe forms of TBI, is known as therapeutic hypothermia and

involves a controlled decrease of a patient's body temperature by $2^{\circ} \mathrm{C}{ }^{163}$. Hypothermia 
has been shown to be neuroprotective in preliminary studies involving severe TBI, and likely works by decreasing activation of the immune system and creation of ROS after injury, as enzymatic activity is slowed by decreasing temperature ${ }^{164}$. It is currently recommended for a number of other diseases, including stroke and heart attack, although its efficacy in mTBI remains unclear ${ }^{163}$. Transcranial magnetic stimulation is another novel treatment being investigated for use in mTBI sufferers who experience long-term negative effects. It involves exposing focal regions of the outer skull to large magnetic fields, which induce a change in current of the underlying brain region. Although this treatment has shown benefit for headaches, it has yet to pass the rigors of a placebo controlled trial ${ }^{165}$. The variety of unconventional treatments being investigated for TBI sequelae serve to highlight the unsatisfactory means with which medicine can currently treat these patients.

\section{Vision treatments}

Treatment for mTBI-associated visual symptoms are primarily aimed at addressing oculomotor dysfunction and double vision in an effort to improve the functional outcome and improve patient quality of life. Oculomotor training is done to strengthen the muscles of the eye in a similar way that physical therapy works after surgery of a joint ${ }^{166}$. It has been shown to significantly improve symptoms in patients with oculomotor-based difficulties, such as vergence and accommodation problems ${ }^{166}$, but, like physical therapy, requires a large input of time for the patient and can be costly. Another commonly used treatment, called binasal occlusion, involves the patient wearing glasses which obscure the nasal aspect of both left and right visual field through the placement of a light-blocking lens ${ }^{60}$. Binasal occlusion blocks diploplia in the central visual field (the region of vision seen by both eyes), thus alleviating some of the discomfort associated with diploplia, while leaving peripheral vision intact in its binocular form. It is not meant to be used as permanent treatment, but may help to retrain the visual system when used in conjunction with other vision rehabilitation ${ }^{167}$. Binasal occluders are often combined with optic prisms, and can bring a patient's vision up to $20 / 20$ or better in some cases ${ }^{168}$. The neural mechanisms underlying these treatments are not well understood, but result in tangible changes in the visual evoked potential as recorded from the primary visual cortex ${ }^{169}$. One case study used high density visual evoked potential (VEP) to image brain regions activated in a patient with significant vision problems secondary to TBI, both with and without corrective lenses ${ }^{168}$. Without correction, the VEPs were slowed significantly, and indicated widespread activation of many vision and non-vision areas, whereas lens-corrected VEPs demonstrated more efficient activation in the primary visual areas, as would be expected. This inefficiency in visual processing reflects the brain's attempt at integrating memory, sensory, and language information in an effort to complete missing visual input due to the injury.

Overall, no pharmacologic treatment is available to address the complex array of symptoms that occur after mTBI, and even with the system-specific treatments that are efficacious, there remain significant pitfalls. A need exists to develop alternative 
treatment options aimed at dealing with the underlying pathology of TBI that is independent of the initial trauma, namely, the pathology associated with immune activation.

\section{Blast-Associated TBI and the Military}

It is pertinent to briefly discuss the controversy that surrounds blast-associated $\mathrm{mTBI}$, both publicly and in the literature. Blast-induced traumatic brain injury has become an increasingly common occurrence, with the use of improvised explosive devices in modern warfare, and has lead to the labeling of mTBI as the "signature" injury of the Operation Enduring Freedom/Operation Iraqi Freedom (OEF/OIF) wars ${ }^{170}$. However, it remains unclear whether the pathophysiology underlying blast-associated mTBI differs from that of concussion, or other more common mechanisms of acquiring $\mathrm{mTBI}$. There is some evidence that blast-related mTBI is uniquely associated with vision symptoms, which makes it an important concern for the military both now and in the future ${ }^{171-173}$. This is made apparent by the military's commissioning of a special report in 2012 to outline the scope of the mTBI vision problem within the non-civilian military population. In this report, it was estimated that over 5,000 new service members will present each year with with mTBI-associated vision dysfunction ${ }^{174}$, costing an estimated 2.2 billion dollars in annual healthcare cost for treatment and rehabilitation services related to those vision problems ${ }^{174}$. The report also goes on to predict another 24 billion dollars in future healthcare expenditures over the lifetime of affected service members. These numbers highlight the significant need to both understand the complex pathology underlying mTBI vision symptoms, and to find adequate treatment for the thousands of individuals living with this burden.

While the purpose of this dissertation is not to delve in to the differences that may or may not exist in the pathophysiology of mTBI resulting from blast injury and mTBI from other sources, it is important to note the unique association of blast-related mTBI with visual dysfunction. The debate underlying these potential differences is unlikely to be settled any time in the near future, as many political and social institutions would probably frown on the conclusion that a cornerstone of modern warfare, the explosive device, is leaving indelible and seemingly invisible wounds on many U.S. veterans. It is with this in mind that we will go on to discuss the specifics of the model used in this dissertation, the pathology it presents, as well as a potential therapeutic intervention using the body's own immune system to curtail secondary damage of TBI. 


\section{CHAPTER 2. PRIOR PUBLISHED STUDIES ON A MOUSE MODEL OF MTBI AND THE DRUG SMM-189}

\section{Mouse Model Overview and Justification}

For this dissertation, we used a mouse model of closed-skull overpressure blastinduced mild traumatic brain injury that causes distinctive, replicable pathology in the whole brain and visual system. The histopathologic and functional endpoints replicate features of mild traumatic brain injury and associated visual dysfunction seen in humans, and are positively correlated with blast pressure amplitude. This chapter will give an overview of previously published data using this model, and the experimental drug SMM-189.

In this model, C57BL/6 mice were exposed to a controlled pulse of air at pressures ranging from 20-60 pounds per square inch (psi) to the left temporal aspect of the cranium. The eye, ear, and body were shielded from the pressure wave, and the mouse's body and head were cushioned. In this way, the injury is focalized, replicable, and prevents any confounding effects of whole-body blast exposure ${ }^{175}$. Furthermore, head movement is severely restricted, in an effort to negate effects from lateral acceleration-deceleration injury. This model was adapted from a published ocular blast model using the same apparatus to administer direct ocular injury in mice ${ }^{176-178}$, and has been extensively described and validated in previous publications ${ }^{179-181}$. The following sections will give brief descriptions of the extensive characterization that has been done of the model, as well as the main histopathologic and functional findings that have previously been described in the literature. Further information on the blast device, blast wave characterization, and blast administration will be found in the Methods section.

\section{General health and survival}

Survival studies in this model have found relatively low mortality rate at psi exposures producing mild injury ( $>98 \%$ below $50-p s i)$, which is consistent with human cases of mild brain injury ${ }^{16}$, while 70 -psi exposure results in almost invariable death. A 60-psi exposure gives indications of mild-moderate brain injury, and survival rate is slightly lower at $91.5 \%$. Animals who die as a result of pressures ranging from 50-70 psi show widespread intracranial and subdural bleeding, indicating that at these pressures, death most likely results from acute intracranial bleed due to blood vessel compromise. For the purposes of this dissertation, it was deemed important to have a higher survival rate, and so a blast psi of 50 was chosen.

Blood oxygenation, respiratory rate, and heart rate were measured acutely after blast in and did not differ between sham and 50-psi blasted mice. Importantly, intraocular pressure measurements between 0- and 50-psi animals also did not differ acutely after blast. Subsequent gross examination also found no signs of corneal edema. These two findings confirm that there was no direct damage to the globe of the eye, as 
direct ocular injury is known to result in an increase in intraocular pressures and edema acutely after blast ${ }^{176}$. Weight was also measured both acutely and long-term after blast, with no significant effects between 0- and 50-psi blasted animals. All of these findings speak to the relatively mild form of brain injury occurring in this model.

\section{Behavioral endpoints}

Mild TBI and concussion are known to result in subtle, albeit evident deficits in

gross motor function acutely after injury ${ }^{54}$. To investigate motor function in this model, mice subjected to varying pressure levels were tested on rotarod, open field, and digigait at multiple time points after blast. See Figure 2-1 for a summary of all of these findings. Animals were grouped according to blast level, with 0-20 psi in one group, 25-40 psi in another group, and 50-60 psi in the final group. Two weeks after blast, the $0-20$ psi group showed no significant differences on rotarod performance from the 25-40 psi group. Early on, the 50-60 psi group showed large deficits in rotarod performance when compared to the other two groups, however this difference subsided at two weeks after blast. Open field assessment found a similar pattern, with the 25-40 psi group only showing marginal differences from the 0-20 psi group at all time points after blast, but the 50-60 psi group showed persistent deficits compared with the other two groups. Unlike with rotarod, these effects were present even at 2 weeks after blast. Finally, a small defect in gait symmetry was found in the 50-60 psi animals compared with 0-20 psi animals at 3 weeks after blast, showing persistent motor deficits at a sub-chronic timepoint. These motor effects may be related to histopathology of the corticospinal tract that was subsequently identified in these animals, detailed later in the histopathology section.

\section{Functional vision endpoints}

Visual acuity (VA) is a measurement of the eye's resolving power- the ability to distinguish two points as being separate from one another, and is given as a measurement of cycles per degree (c/d), or spatial frequency. Contrast sensitivity (CS), on the other hand, is the contrast required to achieve a certain resolving power, that is the amount of contrast required to distinguish two points as separate from one another. This is usually given as a percent of contrast required to resolve the two points, with $100 \%$ meaning full contrast (black and white) and $1 \%$ meaning very little contrast (white and light grey). These two fundamental measurements can serve as a basic readout of visual function in many retinal diseases ${ }^{182}$. In mice, VA and CS thresholds can be acquired behaviorally in awake animals by observing gaze-evoked horizontal nystagmus in response to visual stimuli using a device known as an Optomotry system (see Methods section for a greater discussion of this device) ${ }^{183}$. In our model, mice were subjected to varying blast pressures and then tested for VA and CS at 3 weeks after blast to obtain a readout of visual function. Using the previously described groupings of 0-20 psi, 25-40 psi, and 50$60 \mathrm{psi}$, left and right eye measurements of visual acuity were compared. Neither eye of the $25-40$ psi group differed significantly from the $0-20$ psi group. However, there was a 


\section{Figure 2-1. The effect of blast on motor performance.}

This figure compares the effects of $0-20$ psi blasts, $25-40$ psi blasts, and 50-60 blasts on Rotarod motor performance (A), and a series of motor end-points in open field (B-H). Note that motor performance is impaired for 50-60 psi mice on most parameters (other than acceleration), but for very few parameters in the case of 25-40 psi blast (notably distance in open field). Red asterisks at each time point indicate a significant difference between 50-60 psi mice and 0-20 psi mice, while a red asterisk to the right indicates a significant overall difference between 50-60 psi mice and 0-20 psi mice. Red pound signs at each time point indicate a significant difference between 50- 60 psi mice and 2540 psi mice. Green asterisks at each time point indicate a significant difference between 25-40 psi mice and 0-20 psi mice, while a green asterisk to the right indicates a significant overall difference between 25-40 psi mice and 0-20 psi mice. Reprinted with permission. Guley, N. H. et al. A Novel Closed-Head Model of Mild Traumatic Brain Injury Using Focal Primary Overpressure Blast to the Cranium in Mice. J Neurotrauma 33, 403-422 (2016). 

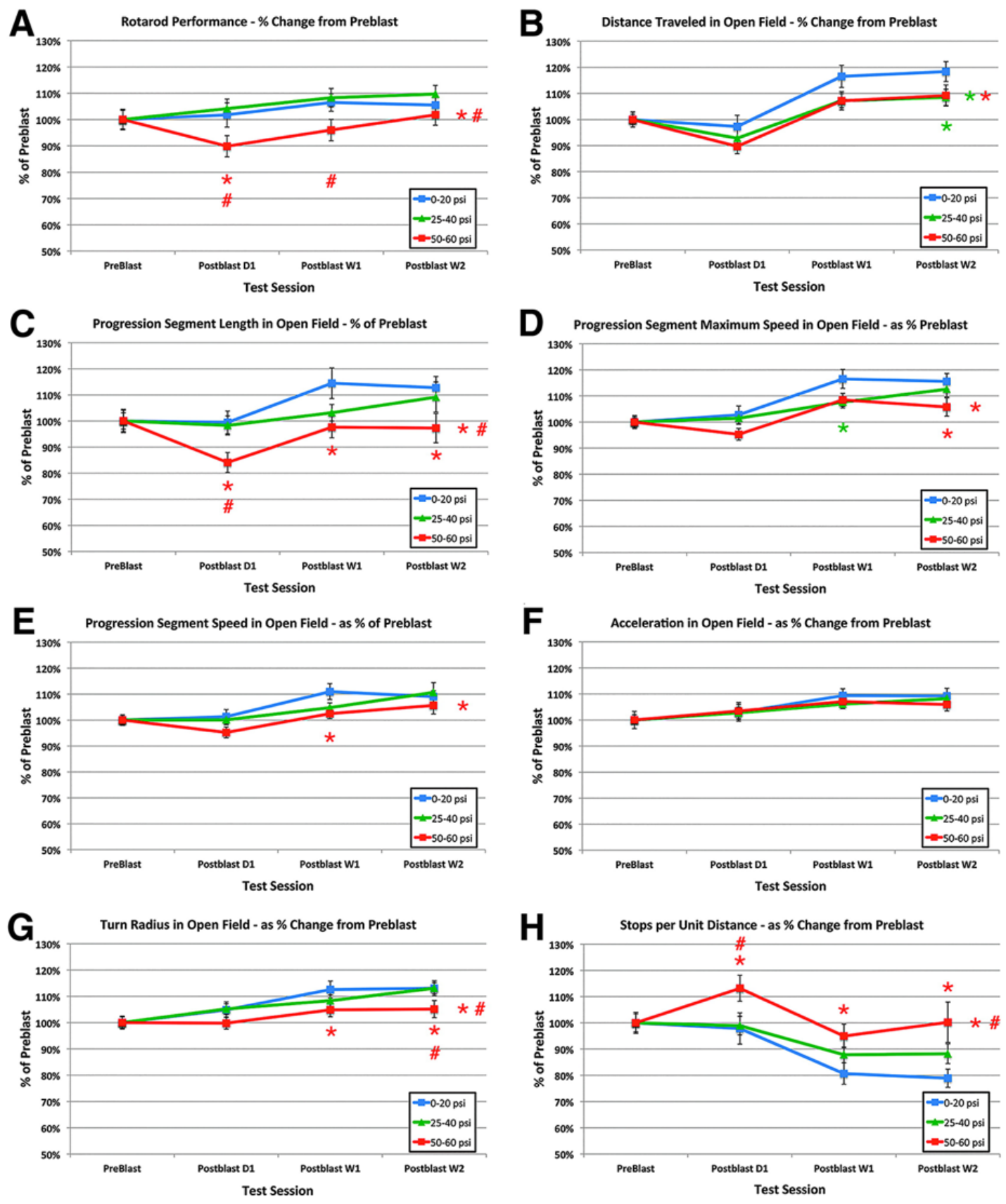
large decrease in both the left eye (25.3\%) and right eye (43\%) visual acuity in the 50-60 psi group when compared to the $0-20$ psi group. Contrast sensitivity measurements were then acquired for both the $0-20$ psi and the 50-60 psi groups at a spatial frequency of $0.042 \mathrm{c} / \mathrm{d}$. In the left eye of 50-60 psi mice, there was a significant increase in the contrast required to detect $0.042 \mathrm{c} / \mathrm{d}$ when compared with $0-20 \mathrm{psi}$ animals, $86.6 \%$ versus $44 \%$, respectively. The right eye of 50-60 psi animals was similarly, but less severely, affected, with a CS threshold of $68.3 \%$. See Figure 2-2 for a summary of these findings.

\section{Histopathologic findings}

General. On gross inspection, brains from blasted and non-blasted animals showed no overt signs of pathology, but were subsequently weighed, then dehydrated and re-weighed. Using this procedure, it was found that a small, but statistically significant, amount of brain edema was present in 50-psi animals 1 day after blast. Brain edema is also a common finding in human neurotrauma ${ }^{102}$. This finding was confirmed by volumetric measurements of histology sections from the forebrain of 50-60 psi animals 2 weeks after blast, showing a bilateral enlargement of $11.6 \%$ over control animals. This edema was slightly lessened at 3-6 weeks after blast, with a still significant increase in size of $7.7 \%$ over control animals was found. SMI-32 immunohistochemistry revealed no overt or microscopic histopathology, as indicated by blinded densitometry of the area occupied by immunoreactive neurons in the somatosensory and cingulate cortex. However, stereological NeuN + neuron counts revealed a bilateral decrease in total neuron number in the cerebral cortex of 60-psi animals 45 days after blast. Neurosilver stain for degenerating axons and terminals also revealed diffuse silver-impregnated fibers throughout many white matter tracts, including the medial and lateral lemniscus, pyramidal tract, and cerebellar peduncles. This pattern of axonal injury is pathognomonic for DAI found in human forms of mTBI ${ }^{85}$.

In the spinal cord, the corticospinal tract was visualized using an antibody against PKC- $\gamma$, and then measured at the level of the mid thorax. The right dorsal component of the corticospinal tract was found to be atrophic, $10 \%$ smaller than the left side, in 60 -psi animals 1-2 months out from blast. This is consistent with left sided upper motor neuron damage, since the corticospinal tract decussates at the more rostral spinomedullary junction, and continues caudally on the contralateral side.

Visual areas. The most prominent histopathology in this model is found in both the central and peripheral visual areas, which makes it an excellent model in which to study TBI-associated visual dysfunction. In this section, we will discuss all of the findings relating to the visual system from proximal to distal, starting in the brain and ending in the eye. These histopathologic end points include evidence of significant white matter damage, inflammation, and subsequent axonal degeneration within areas central to vision and visual processing. It is believed that these injuries directly translate into the functional vision deficits described in a previous section. 

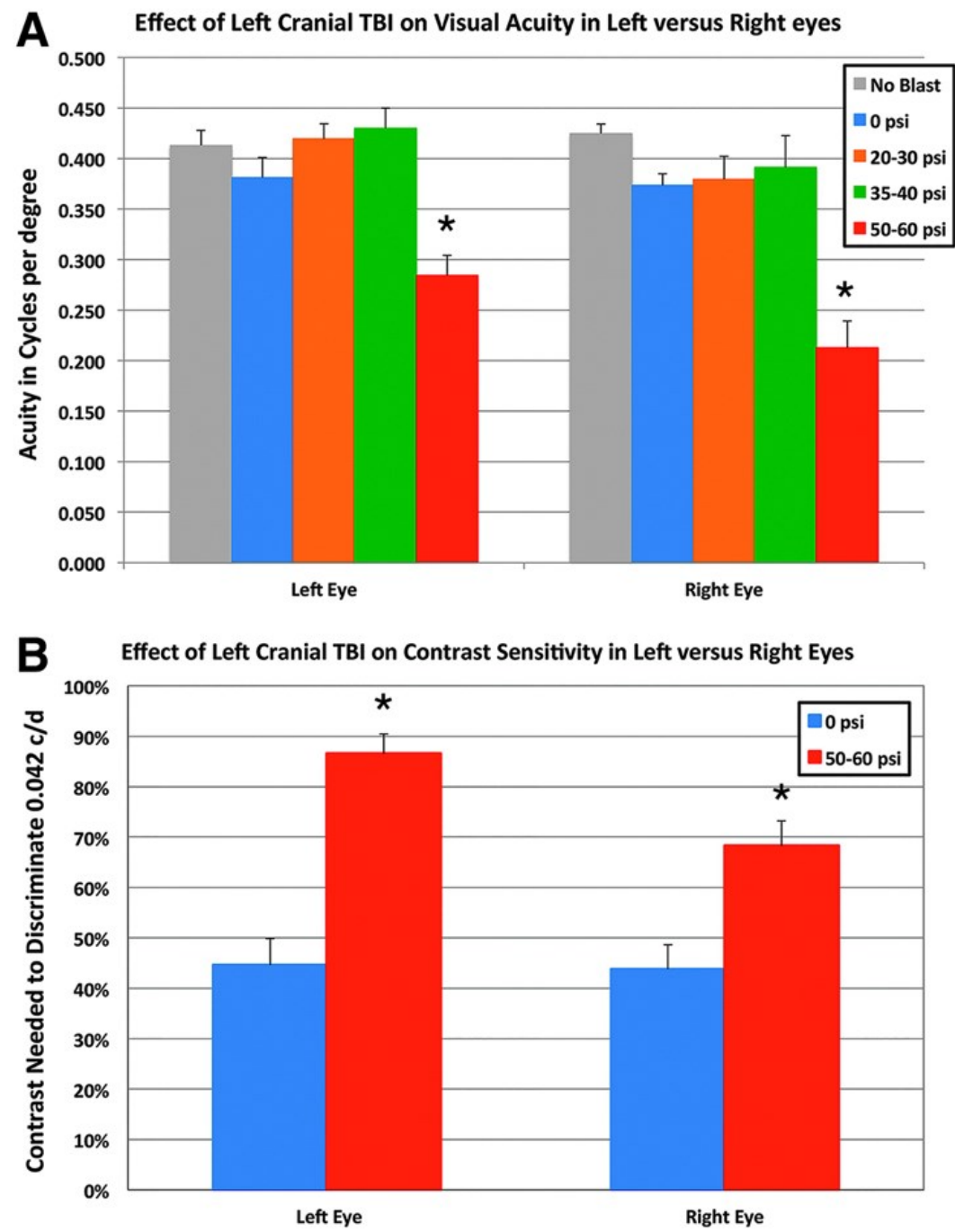

Figure 2-2. The effect of blast on the visual system.

This figure shows the effect of blast on visual acuity (A) and contrast sensitivity (B). (A) Optomotor visual testing in mice with 20-40psi blast revealed normal visual acuity measured at $100 \%$ contrast in both eyes compared with sham blasted or normal mice. Visual acuity in both eyes of 50-60 psi blast mice was diminished (B). Contrast sensitivity was also significantly reduced in 50-60psi mice compared with 0-psi micethat is, the 50-60psi mice needed more contrast to discriminate $0.042 \mathrm{c} / \mathrm{d}$ than did sham mice. Asterisks indicate significant differences between 50-60 psi and sham (0-psi) mice. TBI, traumatic brain injury.

Reprinted with permission. Guley, N. H. et al. A Novel Closed-Head Model of Mild Traumatic Brain Injury Using Focal Primary Overpressure Blast to the Cranium in Mice. J Neurotrauma 33, 403-422 (2016). 
Within the brain, evidence of significant axonal disruption was found beginning 3 days after blast and lasting until 7 days after blast in 60-psi animals. EYFP reporter mice were used to visualize axonal morphology in a subset of retinal ganglion cell axons running through the optic chiasm and tract, while C57BL/6 mice were examined in the same areas using SMI-32 immunohistochemistry. Both EFYP and SMI-32 showed regions of bulbous axolemmal swelling, indicative of microtubule breakage and subsequent disruption in axonal transport. These were evident in the right OT, and to a lesser extent in the left OT. Axons in the right OT are predominantly from RGCs in the left eye, since nearly all of the axons in the optic nerve decussate at the optic chiasm to continue on the contralateral side as the optic tract in the brain. Axon bulbs were quantified and found to peak at 3 days, then decrease by nearly half at 7 days, before returning to control levels two weeks out from blast. This time course is similar to that described in the literature for axon damage and subsequent axonal degeneration by autophagy ${ }^{184,185}$.

Microglia were visualized using antibodies to Iba-1, a protein expressed exclusively in monocytes, and an antibody called OX6 against MHC II. Note that M1 reactive microglia upregulate $\mathrm{MHC}$ II expression in order to begin antigen presentation. On both readouts, highly reactive microglia were found in the right optic tract, right dorsolateral geniculate nucleus, and right superior colliculus (Figure 2-3), with temporal progression mirroring axon bulb pathology previously discussed. Accordingly, numbers of reactive microglia in both optic tracts returned to control levels by 2 weeks after blast. At a month out from blast, numerous silver-impregnated axons were revealed by NeuroSilver staining, indicating the active degeneration of axons even after other signs of damage had subsided. Again, the quantity of degenerating axons was higher in the right OT than the left, mimicking other pathology seen in the OT.

Plastic embedded cross sections of optic nerves were analyzed and quantified for degenerating axons and total axon number at various levels of blast exposure 2-9 weeks after blast ${ }^{179}$. While animals exposed to lower blast levels (25-40 psi) showed some degenerating axons in the left $\mathrm{ON}$, the right $\mathrm{ON}$ in all blast intensity groups showed very few degenerating axons. However, in animals exposed to 50-60 psi blast, many degenerating axons were seen in the left $\mathrm{ON}$ early on. This number diminished by the later time point and indicated a decrease in active degeneration processes. Optic nerves from 60-psi animals at 10-11 weeks after blast show a slight decrease in total axons $(5 \%)$ of the left $\mathrm{ON}$, but not significantly so.

Finally, retinas were analyzed histologically for signs of pathology using toluidine blue and immunohistochemistry on animals 10 weeks after blast ${ }^{179}$. Retina thickness was measured and found to be significantly decreased compared with controls $(11.6 \%)$ in the left eye of blasted animals exposed to 50-60 psi, with a majority of the thinning being in the inner nuclear layer and photoreceptor layer. Additionally, right eyes of 50-60 psi blasted animals showed significant thickening, perhaps indicative of edema or sclerosis. Iba-1 immunohistochemistry and EFYP reporter mice 3 days after a 60-psi blast exhibited large numbers of axon bulbs at the optic nerve head as the RGC axons exited the globe of the eye, with accompanying reactive microglia. 

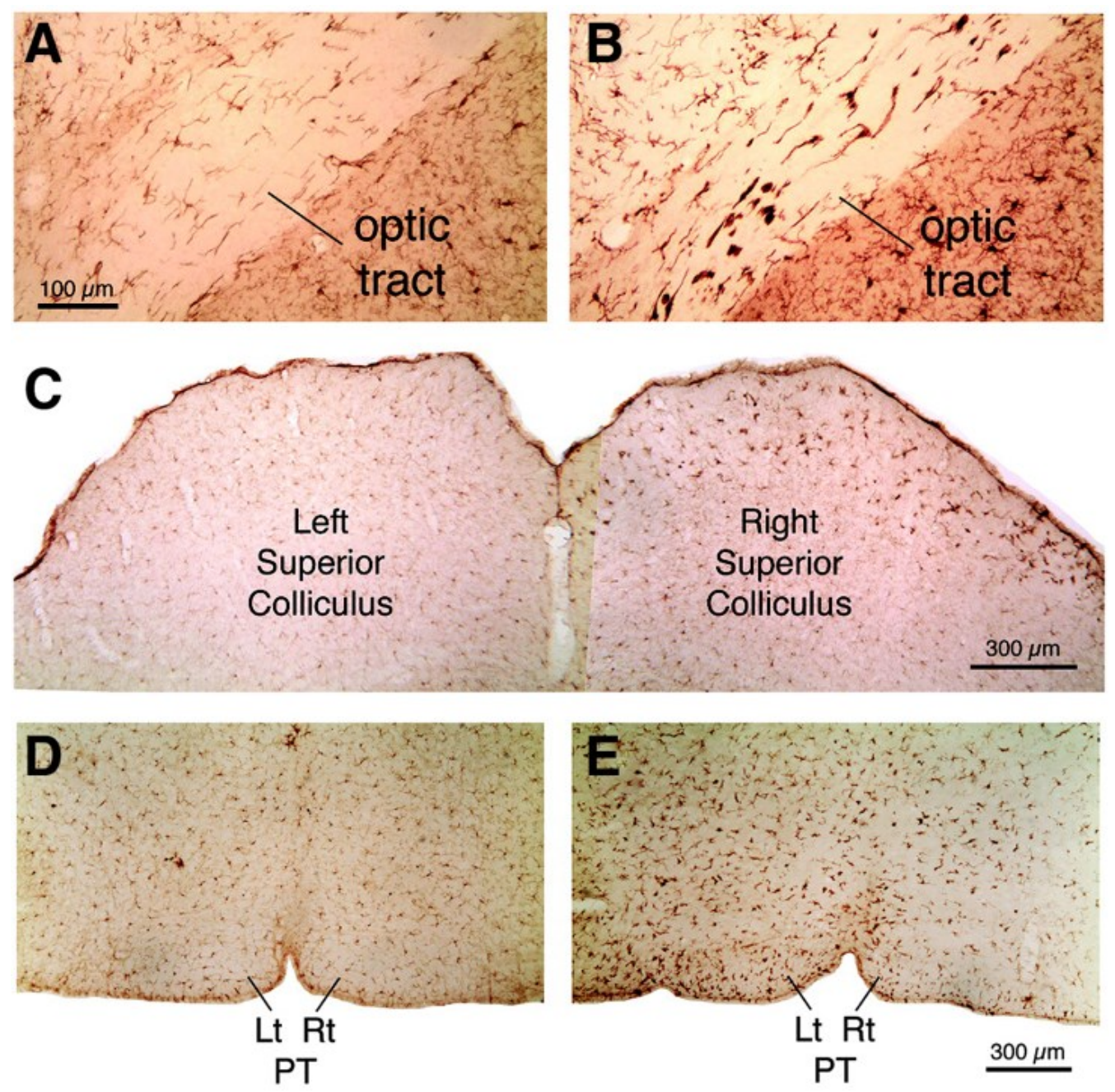

\section{Figure 2-3. Iba-1 immunostaining in the brain of blasted animals.}

This figure shows transverse sections immunostained for the microglial marker IBA1, demonstrating microglial activation in the right optic tract (B), right superior colliculus (C), and left pyramidal (E) tract 3 days after a left cranial 60-psi blast, compared with the right optic tract (A), right superior colliculus (C), and left pyramidal tract (D) 3 days after sham blast. Images $\mathrm{A}$ and $\mathrm{B}$ are at the same magnification as one another, and images D and $\mathrm{E}$ are at the same magnification as one another.

Reprinted with permission. Guley, N. H. et al. A Novel Closed-Head Model of Mild Traumatic Brain Injury Using Focal Primary Overpressure Blast to the Cranium in Mice. J Neurotrauma 33, 403-422 (2016). 
The extensive characterization of this model has demonstrated a reliable pathology with corresponding functional readouts, and replicates pathology that is seen in human cases of TBI-associated visual dysfunction. Much of the histologic evidence just described points to the role of extensive immune cell activation, which may go on to exacerbate the injury and lead to long term disability in individuals with TBI. Thus, this model represents an excellent medium in which to examine potential therapeutic agents, specifically those aimed at modulating or decreasing the secondary injury associated with TBI. The purpose of this dissertation work was to investigate such a drug, one that acts on cannabinoid receptors in the brain and periphery, but before discussing the specific drug, it is pertinent to briefly review cannabinoid receptors and their role in the immune system.

\section{Cannabinoids and the Immune System}

\section{Cannabinoid receptors and their ligands}

Cannabinoid receptors (CB receptors) are ubiquitous throughout the body and central nervous system ${ }^{186}$, with at least 2 different types being known and wellcharacterized - type 1 and type 2. Although these are the most commonly discussed, it is known that many more are likely to exist ${ }^{187}$. Both types of cannabinoid receptors are Gi/o-coupled, and thus inversely coupled to adenylyl cyclase activity, meaning functionally that their activation by an agonist decreases cAMP production within the cell 188. Additionally, their stimulation can activate other protein kinases that are involved in cell survival, differentiation, and proliferation pathways ${ }^{189}$. Importantly, cannabinoid receptors have been shown to exhibit constant low level signaling even in the absence of ligand, a phenomenon known as constitutive activity ${ }^{190-192}$. Others have hypothesized the seeming base level of activation to be a product of tonic endocannabinoid production and subsequent receptor activation ${ }^{193}$. Either reality demonstrates the innate importance of the cannabinoid receptor system in the human body, even under basal physiologic conditions.

Cannabinoid type 1 (CB1) receptors mediate the well-known psychotropic effects of cannabinoids ${ }^{188}$ via agonist activation. They are found predominantly on neurons within the brain and more sparingly in other tissues peripherally ${ }^{192}$, and thus mediate a primarily neurologic function in the body. Cannabinoid type 2 (CB2) receptors share about $44 \%$ homology with CB1 receptors, but are found throughout the body on immune cells and in the CNS primarily on microglia ${ }^{194}$. As their presence on immune cells would imply, CB2 receptors are thought to play a key role in modulating the immune system via the endocannabinoid system ${ }^{192,195}$. Naturally occurring cannabinoids $2-A G$ and anandamide (also called AEA) display complex pharmacology, acting on both CB1 and $\mathrm{CB} 2$ receptors, and interact with every cell type involved in the innate and the adaptive immune response ${ }^{196}$. Indeed, cannabinoid receptors and their endogenous ligands may be critical players in the body's ability to regulate the immune response and 
prevent over-activation when inflammation is no longer necessary ${ }^{196}$, making this system a prime target for therapeutic development.

\section{Cannabinoids as immune modulators}

Phytocannabinoids, such as cannabidiol, have long been recognized for their neuroprotective effects ${ }^{197}$, making them attractive targets for treatment in neurodegenerative diseases that have an underlying immune etiology. Unfortunately, non-selective agonism acting at CB1 receptors, and the subsequent psychotropic effects, represent an unpleasant and usually intolerable side effect when cannabinoids are used for non-recreational purposes ${ }^{198}$. For this reason, modern therapeutic research has aimed at creating synthetic CB2-selective compounds for use in neurologic disease states. Under physiologic conditions, expression of CB2 mRNA in the brain is very low ${ }^{199}$. However, during microglial activation, the expression of CB2 receptors is increased significantly ${ }^{200}$. This receptor expression increase may allow the body to mediate alterations in polarization state in response to the cellular environment by production of endogenous cannabinoid ligands ${ }^{131}$. Other research has pointed to the CB2-mediated modulation of inducible nitric oxide synthase and mitogen-activated protein kinases as an additional source of the neuroprotective effects ${ }^{201}$.

Endogenous and herbal cannabinoids display complex pharmacologic action at CB2 receptors, acting as both agonists and inverse agonists depending on the environmental conditions ${ }^{188,202}$. Agonists directly activate the receptors, inhibiting cAMP production and decreasing cAMP levels intracellularly. Conversely, inverse agonists act to decrease the constitutive activity of the CB2 receptor, and thus have the opposite effect of an agonist - that is, inverse agonists yield an increase in intracellular cAMP. Simple CB2 receptor agonists have been found to increase microglial proliferation, decrease inflammatory cytokine release ${ }^{203}$, and have proven to act as neuroprotectives in some disease models ${ }^{204}$. Paradoxically, CB2 inverse agonists display robust anti-inflammatory actions as well, decreasing leukocyte recruitment in vivo ${ }^{205}$, $\mathrm{NO}$-associated tissue injury ${ }^{206}$, and inflammatory cytokine release from immune cells ${ }^{181}$. These seemingly contradictory findings highlight the true pharmacologic complexity present in the cannabinoid system - with it's dependence on both receptor and ligand concentrations, and the cell types involved ${ }^{195}$. Despite this, it remains clear that CB2 plays an important, albeit poorly understood role, in the regulation of microglial polarization, and that action on CB2 can powerfully modulate immune-mediated damage in injury or neurodegeneration. With this in mind, we will discuss the specific cannabinoid ligand used in this dissertation research.

\section{Investigative drug SMM-189, an Inverse Agonist at CB2 Receptors: Evidence for Its Efficacy}

In this dissertation, we used an investigational drug treatment aimed at modulating the immune response after TBI by action at CB2 receptors, and sought 
functional and morphological endpoints to corroborate its therapeutic potential. This drug, SMM-189, was designed and synthesized by the Bob Moore Group at UTHSC and was generously provided for all of the animal studies described herein. Before detailing the goal and objectives of this dissertation, it is important to describe basic pharmacology of the drug that was used, as well as the preliminary benefits seen in this mouse model that prompted further investigation into its therapeutic potential.

SMM-189 is a novel cannabinoid ligand with a non-classical tri-aryl core that exhibits characteristics of a potent and selective CB2 inverse agonist in vitro ${ }^{207}$. Being highly lipophilic compounds, tri-aryl cannabinoids similar to SMM-189 have demonstrated excellent BBB penetration, and thus characterize a viable drug therapy for targeting to the brain ${ }^{208}$. As described previously, inverse agonists involve a novel pharmacologic mechanism that has demonstrated promise as a potential immunomodulator when selective for $\mathrm{CB} 2$ receptors ${ }^{205}$. Accordingly, in vitro studies were done using immortalized human microglia, and SMM-189 was shown to decrease a multitude of inflammatory cytokines and chemokines in response to LPS stimulation

${ }_{181,209}$ (Figures 2-4 and 2-5). Further, experiments in these cells demonstrated that SMM189 also increased expression of cell surface markers associated with M2 microglia, while decreasing M1-associated surface markers (Figures 2-6 and 2-7). These two groups of in vitro experiments taken together indicate a potentially functionally significant shift in microglial polarization from M1, pro-inflammatory microglia, to the more neuroprotective M2 phenotype by the drug SMM-189.

In previously published studies with our TBI model, mice were blasted with either 0 (sham) or 50-60 psi overpressure blast, then treated with drug or vehicle ${ }^{181}$. SMM-189 was administered intraparitoneally (ip) at $6 \mathrm{mg} / \mathrm{kg}$ beginning 2 hours after blast, and once every day thereafter for 14 days in total. The mice were then subjected to a number of functional tests that were previously described in this dissertation, and a number of notable significant improvements were seen in drug-treated mice, when compared with vehicle-treated mice. Motor, emotional, and histologic end-points not relating to the visual system are summarized in totality in Table 2-1. In addition to motor and emotional improvements seen with SMM-189, significant improvement in the areas pertaining to the visual system were also seen. Optomotry revealed a significant increase in both the left and right eye on visual acuity threshold, as well as an improvement of contrast sensitivity threshold in drug-treated animals when compared to vehicle-treated animals. Histologic analysis of retinas also revealed structural improvements. The total retinal thickness, as well as the inner nuclear layer thickness of left eyes were both increased toward normal in drug-treated animals. In right eyes, the total retinal thickness was not significantly improved toward normal, but the inner nuclear layer was with drug treatment. These results are summarized in Figure 2-8.

\section{Goal and Planned Experiments}

The goal of this research was to investigate visual dysfunction in our mouse model of overpressure blast-induced TBI, and to test the hypothesis that a major pathway 


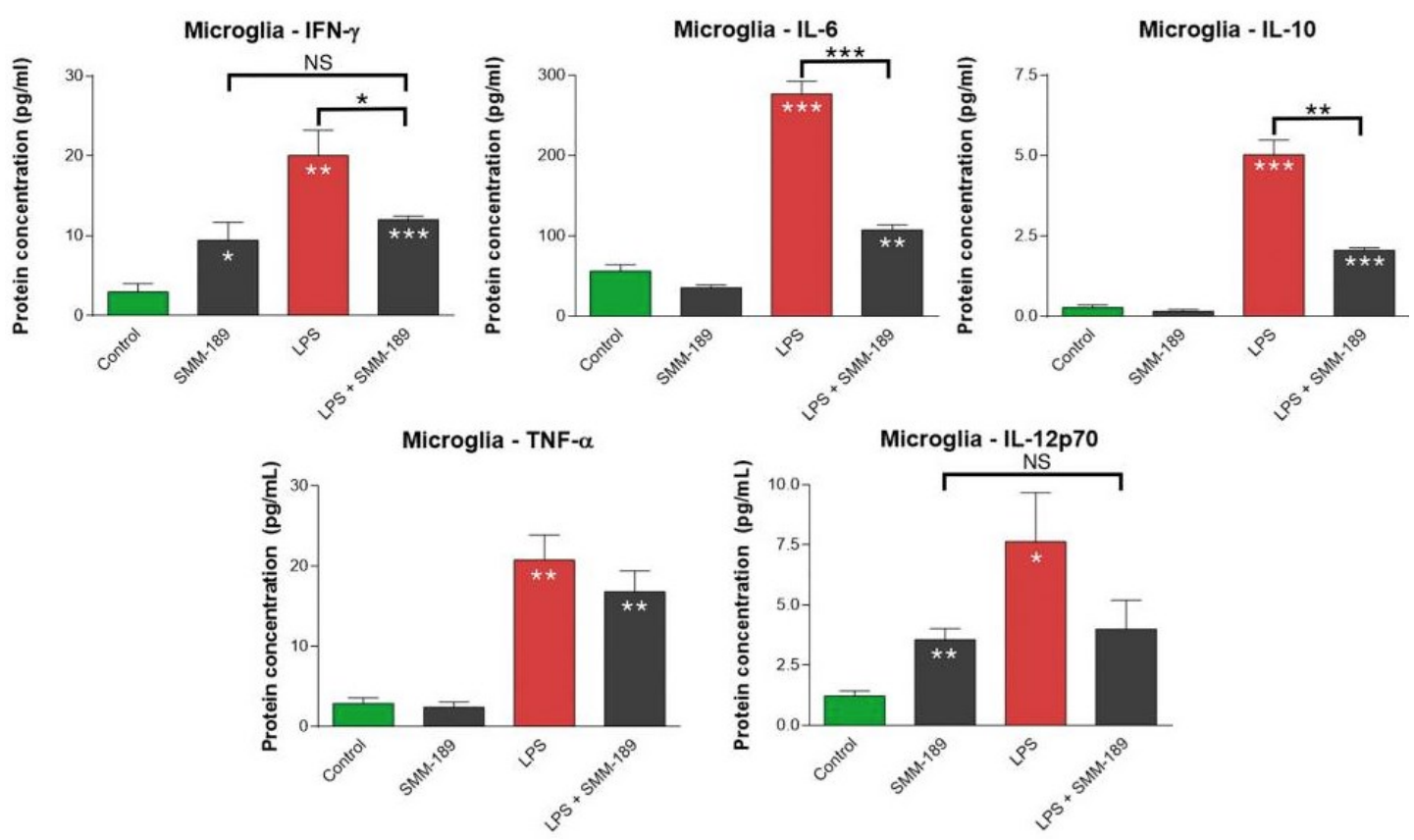

Figure 2-4. Cytokine assay profiles of human microglia in vitro.

This figure shows the effect of SMM-189 on cytokines secreted from primary human microglial cells in vitro 17 hours after treatment with lipopolysaccharide (LPS) or vehicle, and an hour later with SMM-189 or vehicle. SMM-189 by itself significantly elevated interferon-gamma (IFN- $\gamma$ ) and interleukin-12p70 (IL-12p70), but to a lesser extent than LPS. SMM-189 decreased the effect of LPS on cytokine secretion, significantly reducing IFN- $\gamma$, interleukin-6 (IL-6) and interleukin-10 (IL-10) below LPSonly levels and restoring IFN- $\gamma$ and IL-12p70 to SMM-189-alone levels. SMM-189 also trended toward reducing TNF $\alpha$. Asterisks above bars spanning the LPS-alone and LPS + SMM-189 columns, or the SMM-189-alone and LPS + SMM-189 columns indicate significance levels for the comparison between these two conditions. Asterisks on a column indicate a significant difference from the control condition. NS-Not significant, $* \mathrm{p}<0.05, * * \mathrm{p}<0.005, * * * \mathrm{p}<0.0005$.

Reprinted with permission. Reiner, A. et al. Motor, visual and emotional deficits in mice after closed-head mild traumatic brain injury are alleviated by the novel CB2 inverse agonist SMM-189. Int J Mol Sci 16, 758-787 (2015). 

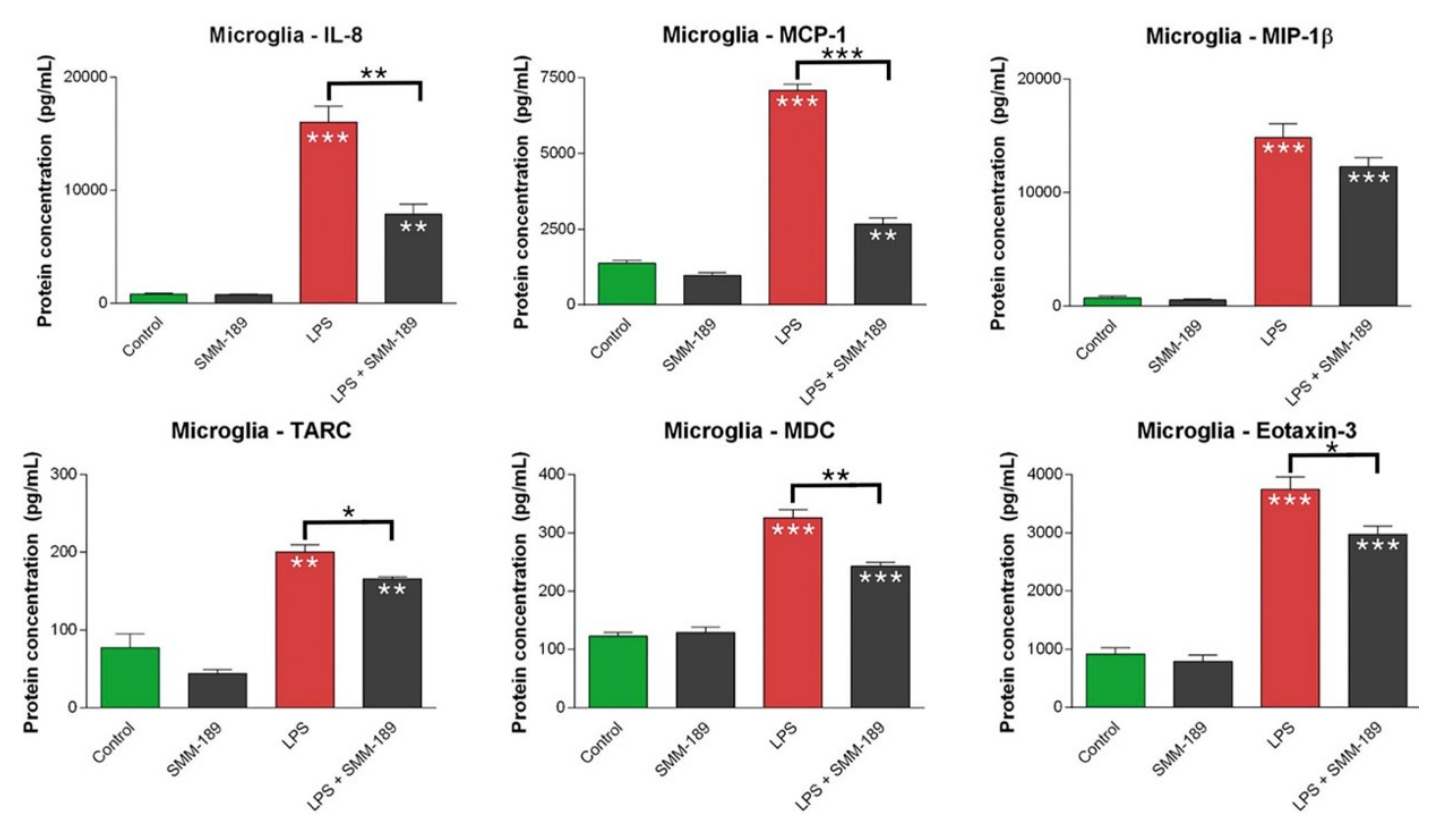

Figure 2-5. Chemokine assay profiles of human microglia in vitro.

This figure shows the effect of SMM-189 on chemokines secreted from primary human microglial cells in vitro 17 hours after treatment with lipopolysaccharide (LPS) or vehicle, and an hour later with SMM-189 or vehicle. LPS significantly increased chemokine levels above those of controls for all chemokines shown. SMM-189 treatment alone had no significant effect on chemokine secretion, other than a slight reducing effect on MCP-1 (monocyte chemoattractant protein-1). SMM-189 significantly reduced the LPS-induced increase in the levels of IL-8 (interleukin-8), MCP-1, TARC (thymus and activation-regulated chemokine, also called chemokine ligand 17, or CCL17), MDC (macrophage-derived chemokine, also called $\mathrm{C}-\mathrm{C}$ motif chemokine 22, or CCL22), and eotaxin-3 (also called C-C motif chemokine 11, or CCL11), and trended toward doing so for MIP-1 $\beta$ (macrophage inflammatory protein-1beta, also called chemokine $\mathrm{C}-\mathrm{C}$ motif ligand 3 , or CCL3). Asterisks above bars spanning the LPS-alone and LPS + SMM-189 columns, or the SMM-189-alone and LPS + SMM-189 columns indicate significance levels for the comparison between these two conditions. Asterisks on a column indicate a significant difference from the control condition. $* \mathrm{p}<0.05, * * \mathrm{p}<0.005, * * * \mathrm{p}<0.0005$.

Reprinted with permission. Reiner, A. et al. Motor, visual and emotional deficits in mice after closed-head mild traumatic brain injury are alleviated by the novel CB2 inverse agonist SMM-189. Int J Mol Sci 16, 758-787 (2015). 


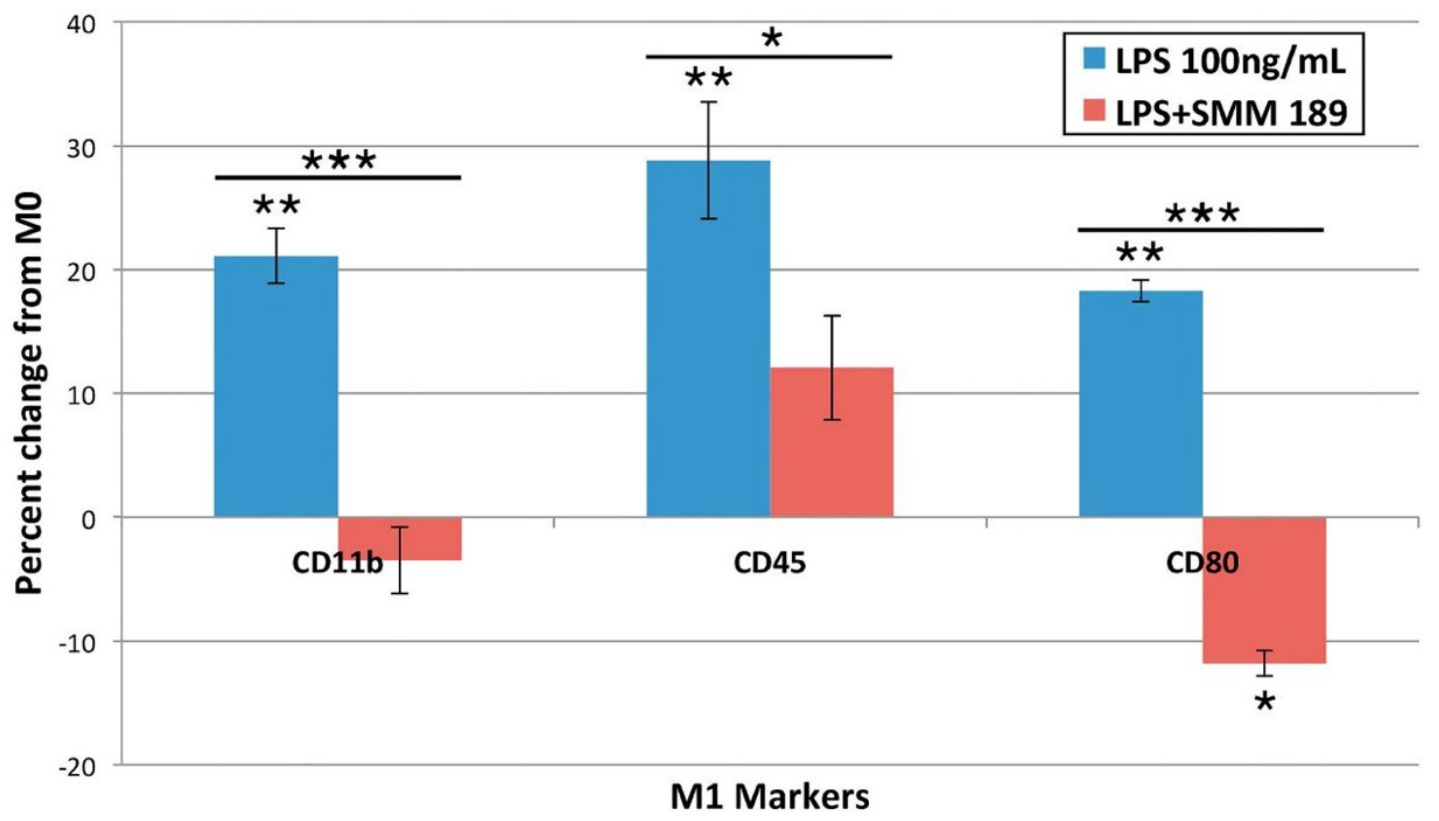

Figure 2-6. Cell surface expression of M1 markers on human microglia in vitro.

This figure shows cell surface receptor expression of M1 microglia phenotype-associated markers CD11b, CD45, and CD80 of human microglia $24 \mathrm{~h}$ after treatment with LPS, or LPS + SMM-189. Levels are expressed as the percent change from control levels (M0). LPS significantly increased expression of CD11b, CD45, and CD80, and SMM-189 significantly reversed expression of M1 markers induced by LPS. Asterisks above the LPS-alone or below the LPS + SMM-189 columns indicate significant differences from the M0 condition. Asterisks above bars spanning the LPS-alone and LPS + SMM-189 columns indicate significance levels for the comparison between these two conditions. * $\mathrm{p}<0.05, * * \mathrm{p}<0.005, * * * \mathrm{p}<0.0005$.

Reprinted with permission. Reiner, A. et al. Motor, visual and emotional deficits in mice after closed-head mild traumatic brain injury are alleviated by the novel CB2 inverse agonist SMM-189. Int J Mol Sci 16, 758-787 (2015). 


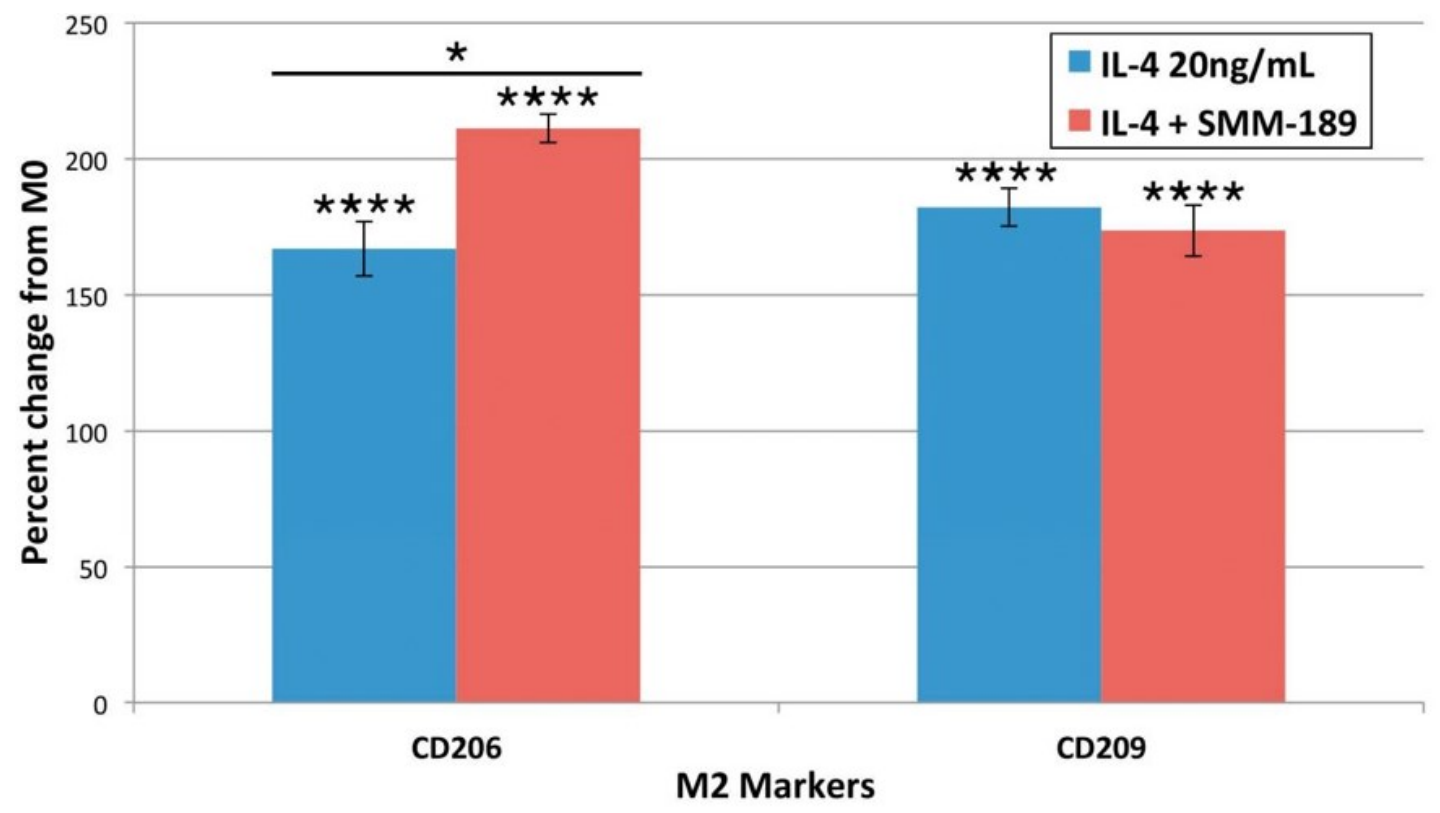

Figure 2-7. Cell surface expression of M2 markers on human microglia in vitro.

This figure shows cell surface receptor expression of M2 microglia phenotype-associated markers CD206 and CD209 of human microglia $24 \mathrm{~h}$ after treatment with interleukin-4 (IL-4), or IL-4 + SMM-189. Note that IL-4 significantly increased expression of both CD206 and CD209, and that SMM-189 significantly increased expression of CD206 beyond that seen with IL-4 alone. Asterisks above bars spanning the LPS-alone and LPS + SMM-189 columns indicate significance levels for the comparison between these two conditions. Asterisks above a column indicate a significant difference from the control condition. $* \mathrm{p}<0.05, * * * * \mathrm{p}<0.0005$.

Reprinted with permission. Reiner, A. et al. Motor, visual and emotional deficits in mice after closed-head mild traumatic brain injury are alleviated by the novel CB2 inverse agonist SMM-189. Int J Mol Sci 16, 758-787 (2015). 
Table 2-1. Summary of previous SMM-189 findings in our mouse model of TBI.

\begin{tabular}{|c|c|c|}
\hline Endpoint examined & $\begin{array}{l}\text { Without drug } \\
50 \text { psi versus sham }\end{array}$ & $\begin{array}{l}\text { With drug } \\
50 \text { psi + SMM-189 } \\
\text { versus sham }\end{array}$ \\
\hline \multicolumn{3}{|l|}{ Motor } \\
\hline Rotarod performance & Decreased* & Decreased + \\
\hline Open field- Max speed & Decreased* & -+ \\
\hline Open field- Turn radius & Decreased* & -+ \\
\hline \multicolumn{3}{|l|}{ Emotional } \\
\hline Tail suspension & Increased* & -+ \\
\hline Contextual fear & Increased* & -+ \\
\hline Fear extinction & Decreased* & Decreased+ \\
\hline \multicolumn{3}{|l|}{ Histologic } \\
\hline Amgydala & Decreased* & -+ \\
\hline Right corticospinal tract & Decreased* & -+ \\
\hline
\end{tabular}

* denotes a significant difference between 50-psi and sham group.

+ denotes drug treated group and sham group are statistically equivalent.

- means no change from sham group.

Italics indicate an evident trend. 

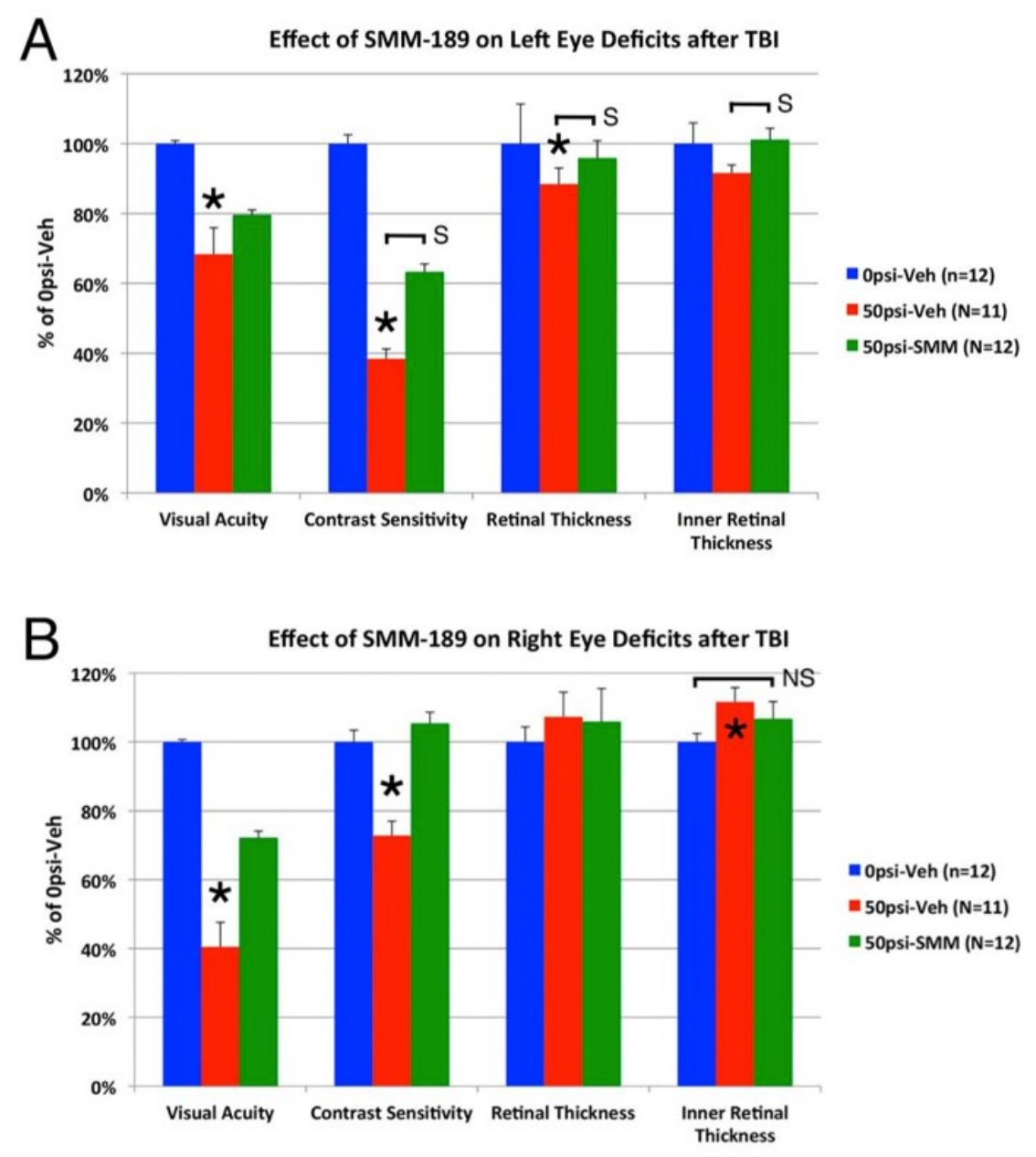

Figure 2-8. The effect of SMM-189 treatment on visual end points.

This figure shows the effect of blast on visual acuity and contrast sensitivity four weeks after blast and retinal thickness 11 weeks after blast in the left eye (A) and right eye (B) of vehicle-treated sham mice, vehicle-treated 50-psi mice, and SMM-189 treated 50-psi mice. Optomotor testing in vehicle-treated mice with 50-psi blast revealed reduced visual acuity and contrast sensitivity in the left eye and retinal thinning (A), compared to vehicle-treated sham mice, which was prevented by SMM-189. Optomotor visual testing in vehicle-treated mice with 50-psi blast also revealed reduced visual acuity and contrast sensitivity in the right eye and retinal thickening, compared to vehicle-treated sham mice (B), which were attenuated by SMM-189. Asterisks indicate significant differences between vehicle-treated 50-psi and sham mice, and bars indicate significance (S) or nonsignificant differences (NS) between the groups spanned by the bar. ${ }^{*} \mathrm{p}<0.05$.

Reprinted with permission. Reiner, A. et al. Motor, visual and emotional deficits in mice after closed-head mild traumatic brain injury are alleviated by the novel CB2 inverse agonist SMM-189. Int J Mol Sci 16, 758-787 (2015). 
of the disease is immune-mediated secondary injury that can be remedied with the use of a targeted immune modulating drug. Specifically, we sought to: A) further characterize the visual histopathology in this mouse model with additional structural and functional end points, and B) explore and characterize the improvements in visual function after SMM-189 administration. To achieve these two goals, experiments were planned which will be further outlined in the following section. The discussion of these experiments is organized by designation as a structural or functional investigation.

\section{Structural endpoints}

In order to test the hypothesis that microglial activation plays a significant role in the visual injury after the initial mechanical insult, and that this mechanical injury itself was unlikely to be amenable to amelioration by the drug, it was necessary to quantify both microglia and axon bulbs in blast-vehicle, and blast-drug treated animals. Axon bulbs in the optic tract were quantified in EYFP reporter mice with and without blast injury, to compare drug treated with non-drug treated animals. Using an antibody to Iba1 as described previously, microglia in the same areas in treated and untreated blasted and sham mice were also counted and categorized as either reactive or resting based on morphologic characteristics, such as retracted processes, intense staining, and amoeboid appearance. The number of axon bulbs was then correlated with the number of morphologically reactive microglia.

In the retina, it was predicted that more extensive pathology should be present beyond retinal thickness changes, in the form of an inflammatory response, and that this inflammatory response should be diminished by SMM-189 administration. Microglia were again identified using an antibody to Iba-1, and then quantified by counting. Glial fibrillary acidic protein (GFAP), a marker of Müller glia in the retina, was used to identify additional signs of inflammation by other resident glia. This was quantified by counting Müller cell processes that penetrated past the RGC layer in the retina ${ }^{210}$. Since this model is known to demonstrate signs of axonal degeneration originating from the RGCs in the retina, we proposed that there should be a decrease in the number of RGCs, since RGC loss is a potential ${ }^{211}$, but not invariant ${ }^{155}$, feature of RGC axonal injury. To visualize RGCs, retinal cross sections through the horizontal meridian were taken and stained with an antibody to Brn-3a, a protein found in a large subpopulation of RGCs 212,213 . Additionally, a possible localization of injury could be extrapolated by looking at early time points, and examining the retina, optic nerve, and optic tract in each animal. In this way, one might be able to determine if the injury of axons in this model is primarily focal or diffuse, and if focal, whether the injury occurs proximal or distal in the visual pathway. Retinal sections of EYFP mice were examined at two early time points, 3 and 7 days, in order to investigate a potential time course.

Signs of axonal degeneration and eventual loss were described in this model previously. If SMM-189 acts on the immune system to curtail subsequent immune damage to these injured axons, one would expect to see an increase in the total axon counts of drug treated animals compared with vehicle treated animals. To determine if 
there is axonal rescue due to drug, optic nerve cross sections were counted manually, and measured for total axonal cross sectional area.

If SMM-189 is indeed acting to alter microglial polarization, switching it from a neurotoxic to a neuroprotective phenotype, this should be reflected in specific surface protein expression by microglia in affected tissue. To examine whether M1/M2 polarization ratios were altered in optic tract of injured animals, immunohistochemistry using antibodies to M1 (CD16/32) versus M2 (CD206) markers were used ${ }^{131}$. Cells were imaged on CLSM, and then quantified according to polarization state.

Finally, non-histologic structural investigations in live animals were performed to obtain pre-blast and post-blast comparisons of individual subjects. Preliminary studies indicated that retinal thinning of the left eye occurs histologically at eleven weeks postblast in these animals, with a corresponding thickening of the right retina. Retinal thickening can be acute, due to an inflammatory process causing edema, or can manifest as a chronic sclerotic lesion, whereas loss of RGCs or support cells might manifest as thinning of one or more cell layers in the retina. Optical coherence tomography (OCT), a minimally invasive imaging method that uses optical scattering to visualize the cornea, lens, and retina in vivo, was used to perform retinal measurements on live animals. This was done once pre-blast, and then again at a month out from blast. Measurements of the outer retina, inner retina, total retinal thickness, and nerve fiber layer thickness were performed, with special attention paid to signs of retinal pathology (such as detachment, etc.). In a small subset of animals, post-blast fluorescence-assisted angiograms were obtained, to look for additional signs of retinal pathology at two weeks after blast.

\section{Functional endpoints}

To assess the functional visual parameters, and how they are affected by SMM189 administration, both optokinetics and electroretinograms were performed. Visual acuity measurements were again performed using the Optomotry device, as previously described, on animals treated with drug and with vehicle. These were done pre- and postblast, in order to gauge individual subjects' visual effects after blast more accurately. Contrast sensitivity thresholds were obtained at 6 spatial frequencies, instead of the 1 frequency used previously, in an effort to gain sensitivity to both CS deficits and improvements due to the drug. The electroretinogram (ERG), a measurement of the electrical conductance across the cornea, was used to assess the function of cells within the retina in animals both pre-and post-blast. Based on the hypothesis that the thinning of the INL and photoreceptor layer of blasted animals should yield some electrophysiological defect in those cell populations as well, ERG waves were analyzed with special attention paid to A- and B-wave amplitudes and latency time. ERG waves are well characterized, and highly correlated with the function of specific cell populations under both normal and pathologic conditions ${ }^{214}$. A-waves correspond to photoreceptor function, while B-waves correspond to functioning of cells in the INL and RGCs ${ }^{215}$. These waves are variably affected in a number of visual system diseases that display retinal pathology, including $\mathrm{TBI}^{216,217}$, and were hypothesized to show a decrease in 
amplitude or latency time, or both, due to the injury. This defect was further expected to be ameliorated by drug treatment, with corresponding improvement in retinal histopathology.

A small group of $4 \mathrm{x}$ multiple blast animals not receiving drug or vehicle treatment were examined at 1 year after blast on optokinetics. Both VA and CS thresholds at 1 spatial frequency were obtained, to determine the effect of multiple blast exposure in this model. 


\title{
CHAPTER 3. GENERAL METHODOLOGY*
}

\begin{abstract}
Animals
Most experiments in this dissertation were done using C57BL/6 mice from Jackson Laboratories (Bar Harbor, ME), with an age of 11 weeks at the time of blast administration. Occasionally, mice were also used from a colony kept by our lab at the University of Tennessee Health Science Center (UTHSC) bred from C57BL/6 founders also from Jackson Laboratories. These mice were involved in the functional vision tests (Chapter 4), as well as the structural analysis performed at one month after blast (Chapter 5). Only males were studied to simplify the experimental design and to maximize numbers of animals in each time point. Additionally, this model has only been extensively characterized in male mice.

Where noted, EYFP-reporter mice were used at shorter time points - 3 and 7 days after blast administration - and were used primarily for structural studies (Chapter 5). These mice were obtained from a cross between a floxed Thyl-EYFP reporter mouse and an emx1-Cre driver mouse, whose offspring express EYFP in all Thy1+ cells of the emx 1 lineage. Although emx1 has not been reported to be expressed in the retina, we found prior to these experiments that about $5 \%$ of retinal ganglion cells also express EYFP, in this way we were able to use these mice to directly image morphology in a subset of retinal ganglion cell axons. The floxed Thyl-EYFP reporter mice (purchased from JAX) and emx1-Cre driver mice (purchased from the Mutant Mouse Regional Resource Consortium, or MMRRC) were maintained as colonies at UTHSC. A group of C57BL/6 mice were used to quantify axon numbers in the optic nerve, and they ranged in survival time from 2 to 11 weeks after blast.
\end{abstract}

\section{Blast device description and characterization}

The blast device described in this model and used for the experiments described in this dissertation is composed of a modified, horizontally mounted paintball gun which administers a controlled bolus of high pressure air to the left lateral side of the mouse's head, at a defined region halfway between the ear and the eye of the mouse. It was designed in corroboration with a fluid dynamics engineer at University of Memphis (Jeffery Marchetta, Ph.D; please see acknowledgements). Extensive characterization has been done of both the blast device, the blast wave emitted from the gun, as well as the biophysics of mouse head movement during blast administration ${ }^{179}$.

*Adapted with permission. Guley, N. H., Rogers, J. T., Del Mar, N. A., Deng, Y., Islam, R. M., D’Surney, L., Ferrell, J., Deng, B., Hines-Beard, J., Bu, W., Ren, H., Elberger, A. J., Marchetta, J. G., Rex, T. S., Honig, M. G., \& Reiner, A. (2015). A Novel Closed-Head Model of Mild Traumatic Brain Injury Using Focal Primary Overpressure Blast to the Cranium in Mice. J Neurotrauma, 2015 Dec 17;:neu.2015.3886. 


\section{Blast cannon}

The blast device is composed of a paintball gun (Invert Mini, Empire Paintball, Sewell, NJ) modified with a custom-machined converging-diverging nozzle which chokes the air flow to achieve higher velocity and higher pressure output than is normally delivered by a paintball gun. The paintball chamber is removed, and the gun emits only a high pressure, high velocity bolus of normal air provided by the pressurized air canister attached to the gun, delivered through a $6.5-\mathrm{mm}$ nozzle opening. The gun itself is attached horizontally to a fixed stage and is aimed at an outer PVCC tube which is affixed perpendicular to the gun nozzle on a moveable $x-y$ stage. The outer tube has two 7.5-mm holes, one that faces the gun nozzle, and another exactly opposing it that allows for insertion of the pressure sensor (used for calibration purposes, described below). A smaller diameter inner tube is used to contain the mouse, and has a larger 11-mm window used to expose the entire left side of the mouse's head. The large inner window is aligned with the smaller outer window, so only a $7.5 \mathrm{~mm}$ diameter head region is exposed to the blast.

\section{Mouse sling}

A flat piece of plastic lined with a thin layer of foam was used to secure the mouse for insertion into the inner tube for blast administration. At the end of the sling where the mouse's head rests, thicker foam pieces were placed on the bottom and top, as well as the front, to further cushion and limit the mouse's head from moving. A tab affixed to the head end of the sling assists with its insertion into the inner tube. During blast administration, the mouse was placed on the sling with the right side of its body fully contacting the foam, and was then taped around the mid-abdomen directly to the sling to prevent movement during insertion and blast administration.

\section{Calibration of blast device}

Just prior to each blast session, the blast device was calibrated for the desired pressure output. A model STJE Sensotec high pressure transducer (Honeywell, Morristown, NJ) was inserted into both the inner and outer tube through holes directly opposing the nozzle of the gun, and was brought to the same location that the mouse's head occupies during blast administration (about 4-5 $\mathrm{mm}$ from the gun nozzle tip). A custom-written program for Labview software (National Instruments, Austin, TX) runs the transducer at a sampling rate of 1000 samples/second and allows real-time visualization and plotting of the peak pressure during a blast. By repeatedly firing the gun, visualizing the peak pressure, and adjusting the pressure nozzle on the back of the gun, the pressure was precisely dialed in to within 0.1 psi of the desired peak pressure. Once the desired peak pressure was achieved, a copy of the pressure versus time graph was exported and saved for documentary record. To see a characteristic blast profile from this apparatus, please see Figures 2 and 5 from our previously published paper ${ }^{179}$. 


\section{Administration of Single Overpressure Blast and Post-Blast Recovery}

Mice were first weighed, then given an intra-peritoneal (ip) injection of Avertin (400 mg/kg body weight). Anesthesia depth was gauged by suppression of tail pinch response. Electric clippers were then used to completely shave the left temporal area of the skull, between the eye and the ear. A white marker was used to place a dot directly on the skin at exactly half-way between the eye and the outer auditory meatus. Mice were then secured in the foam sling using a piece of tape wrapped tightly around the abdomen and sling. The sling was then inserted into the inner tube, with the mouse's eye pinched shut prior to insertion of the head (to prevent corneal abrasion during insertion into the tube). The sling was pulled through the inner tube until the shaved region of the mouse's temporal area was fully visible in the large viewing window, but the left eye was still shielded by the tube. This outer tube was then inserted into the inner tube, with the white dot lined up exactly centered in the smaller window in the outer tube. The blast gun was purged no more than 10 seconds before the administration of the blast, by covering the gun aperture with a piece of heavy wood, and then discharging it. This was done to clear the valve of any accumulated fluid volume that would cause variability in the blast pressure being administered. After the purge was completed, the stage and attached tubes were positioned with the gun nozzle inserted into the aperture of the outer tube viewing window completely, such that the end of the gun nozzle was located at 4-5 $\mathrm{mm}$ from the side of the mouse's skull to be blasted. The gun was then discharged, and the inner tube was then immediately withdrawn from the outer tube, and the sling removed. The tape securing the mouse to the sling was cut, and the mouse was placed onto a heating pad for recovery from anesthesia.

After blast administration, mice were placed onto a heating pad to maintain body temperature from anesthesia-induced hypothermia. Once mice began ambulation, they were placed into the common cage with their litter mates. Most mice were recovered and ambulating by 15 minutes after blast. Tylenol was dosed at $35 \mathrm{mg} / \mathrm{mL}$ in the drinking water of all mice for at least 3 but as many as 5 days after blast.

\section{SMM-189 and Vehicle Administration}

SMM-189 is a highly lipophilic $(\operatorname{cog} \mathrm{P}=5.26)$ tri-aryl cannabinoid with good aqueous solubility $(\sim 185 \mu \mathrm{g} / \mathrm{mL}$ at $\mathrm{pH} 7.4)$, and a terminal half-life of $3.03 \pm 0.49$ hours

218. See reference 217, Figure 1 for the molecular structure of SMM-189. The drug SMM-189 was prepared in accordance with previously described methods ${ }^{181}$. Briefly, powdered SMM-189 (purity $>98 \%$ ) was mixed into a vehicle solution which consisted of a 90:5:5 ratio of $0.9 \%$ sterile saline, $5 \%$ Cremophor EL®, and 100\% ethanol. It was mixed on a vortex and filtered through a syringe-driven filter unit at $0.22 \mu \mathrm{m}$. Mice were given an ip injection of either SMM-189 or vehicle at a dose of $6 \mathrm{mg} / \mathrm{kg}$ of body weight beginning at 2 hours after blast. This time point was chosen to begin treatment as it represented a realistic, clinically-relevant scenario for implementation of treatment after mTBI. This was repeated at approximately the same time every day ( \pm 1 hour) for the next 13 days after blast (14 doses in total). Mice were weighed one week after blast to 
adjust doses for changes in weight, which were minimal ${ }^{179}$. SMM-189 dosing was calculated based off a study using a structurally-similar tri-aryl CB2 receptor ligand ${ }^{219}$, and is estimated to deliver $4.6 \mu \mathrm{M}$ of SMM-189 to the brain within hours of the ip injection at this concentration. With the known CB2 affinity ${ }^{207}$, this was deemed high enough to achieve appropriate receptor activation in the brain ${ }^{181}$.

\section{Administration of 4x Multiple Overpressure Blast and Post-Blast Recovery}

For multiple blast studies, animals were administered with blast overpressure exactly as previously described for animals receiving only a single blast. The blast was administered once every 7 days, for 4 times total. The time after blast began after the $4^{\text {th }}$ blast administration.

\section{Animal Sacrifice and Tissue Removal}

\section{Whole body perfusion}

Mice were deeply anesthetized (Avertin; $0.2 \mathrm{~mL} / \mathrm{g}$ body weight), the chest opened, and $0.1 \mathrm{~mL}$ of heparinized saline ( 800 U.S.P. units $/ \mathrm{mL}$ ) was injected into the heart. They were then perfused trans-cardially with $30 \mathrm{~mL}$ of $0.9 \% \mathrm{NaCl}$ in $0.1 \mathrm{M}$ sodium phosphate buffer at $\mathrm{pH} 7.4(\mathrm{~PB})$, followed by $180 \mathrm{~mL}$ of $4 \%$ paraformaldehyde, $0.1 \mathrm{M}$ lysine- $0.1 \mathrm{M}$ sodium periodate in $0.1 \mathrm{M} \mathrm{PB}$ at $\mathrm{pH} 7.4$ (PLP).

\section{Brain collection}

Brains were removed by first decapitating perfused mice, and then removing the entire superior portion of the skull. Great care was paid to preserve the optic nerve and chiasm from damage. The optic nerves were clipped just distal to the optic chiasm, leaving the optic nerves and eyes in situ for subsequent removal. The brain was then placed in PLP overnight to post-fix. The following day, brains were moved into a $20 \%$ sucrose $/ 10 \%$ glycerol solution and were stored at $4{ }^{\circ} \mathrm{C}$. A pin was also inserted longitudinally into the right side of the brains, in order to distinguish left from right, and was left there until the time of sectioning (a minimum of 3 days).

\section{Eye and optic nerve collection}

Eyes were first cauterized at one small site on the temporal side of the cornea to mark orientation, then injected with PLP using a small syringe. They were then removed from the socket antero-superiorly, by first reflecting back the tissue of the eyelid, removing the orbital part of the frontal bone, and clipping away the ocular muscles to release the eye. The optic nerve was left attached to the eye, and they were both placed 
into PLP for 2 hours of post-fixation at $4^{\circ} \mathrm{C}$. After two hours had elapsed, the lens was carefully removed from each eye by making a corneal flap, leaving the cautery mark intact, and then placed in a $20 \%$ sucrose and $0.1 \mathrm{M}$ PB solution. A micro-suture was subsequently placed through the cautery mark to enable identification of temporal and nasal sides during embedding. A separate set of optic nerves used for axon counts were removed from the eye by excision at the optic nerve head, then fixed in $4 \%$ paraformaldehyde $/ 0.5 \%$ glutaraldehyde in $0.1 \mathrm{M} \mathrm{PB}$, to be embedded in plastic. 


\title{
CHAPTER 4. FUNCTIONAL VISION OUTCOMES IN MTBI
}

\author{
Methods
}

\section{Optokinetics}

Optokinetic data were obtained from all C57BL/6 mice at 30 days after blast. In one small cohort of these animals $(n=14)$, pre-blast VA data were also obtained, to allow inter-subject comparison with post-blast test results. For this subset of animals, their post-blast data was compared with their pre-blast data, and this number (a post-test percent of pre-test) was used for statistical comparison. Another cohort of animals was analyzed from a long-term multi-blast study that did not include drug treatment. These animals had been blasted 4 times in total - once a week for 4 weeks - and were tested at a year after blast. Their blast intensities were either 0-psi (sham) or 50-psi.

General information. Optokinetic nystagmus was used to determine both visual acuity and contrast sensitivity thresholds on live mice pre- and post-blast at one month. A Cerebral mechanics OptoMotry system (CerebralMechanics Inc.: www.cerebralmechanics.com) was used for this purpose ${ }^{183}$. This apparatus consists of four LCD computer screens arranged in a box around a central platform. A camera is suspended above the platform on a ceiling that blocks out all external light. The camera relays a top-down view of the subject and the platform to a computer program and monitor being used by the experimenter during testing. The system is designed such that once the mouse is enclosed in the apparatus, the experimenter cannot see the stimulus being presented, and the mouse cannot see external stimuli. The computer program is used to create a virtual barrel displayed on the LCD screens around the platform and subject, with evenly spaced vertical bars of either black or white that rotate about the center of the arena. Spacing and width of the vertical bars dictate the spatial frequency (c/d) being tested. Clockwise bar rotation tests the left eye and counter-clockwise rotation tests the right eye, since the optokinetic response is driven exclusively by temporal to nasal motion. Within the computer program display for the experimenter, the center of the arena is delineated by a crosshair that is moved around by the experimenter as the mouse shifts head positions.

The test was administered in a double-blinded manner, with the experimenter unaware of either the spatial frequency/contrast level or the eye being tested at any given time. Mice were placed onto the mouse platform in the optomotry device and allowed to acclimate for 1-2 minutes, or until the mouse was calm and still. During the acclimatization period, the screens all displayed a solid grey background, a color that was identified by the system vendor as being the most calming for C57BL/6 mice.

The CerebralMechanics computer program uses a staircase method for achieving a stimulus threshold, where it first presents the lowest possible stimulus $(.003 \mathrm{c} / \mathrm{d}$ in this case), waiting for a positive response (a "yes"), and then moves to successively higher 
stimuli until a negative response is reached, at which time it returns to a lower threshold and repeats the process again. This is done for both counter-clockwise grating movement and clockwise grating movement at the same time, randomly switching which direction is tested to keep the experimenter unaware of which eye was being tested at each stimulus round. A threshold is achieved at which point the step higher is not visible, but the step lower is consistently visible to the test subject. If at any point during testing the subject responded negatively to 7 consecutive stimuli to which it previously responded positively, the test was ended automatically by the computer program. This would occur when the test subject became bored or tired of the stimuli, and ceased to respond regardless of whether the stimuli was actually visible to it or not. If at any point the test subject became unresponsive or hyper-responsive and it was obvious that the subject was under distress or inattentive, it was removed by the experimenter and placed back with its litter mates to be tested at another time. Occasionally it was necessary to flash either a black or a white screen at the test subject to keep it awake and attentive; occasionally knocking and/or whistling was also used for this purpose at the experimenter's discretion.

Visual acuity test administration. For achieving the visual acuity threshold at $100 \%$ contrast, the system was set to the "visual acuity" program, and after the acclimatization period, the experimenter began the test by positioning the crosshair of the computer mouse directly over the area where the subject's eyes were located. In this way, the computer program is able to create a virtual barrel using the crosshairs as the center location for the virtual barrel to turn about. As soon as the experimenter released hold of the computer mouse, the stimulus was presented to the subject, and the experimenter watched the subject for any head movement. If head movement was not detected for 5 consecutive seconds during which the subject's body was completely still, the experimenter chose "no" as a response to the stimulus. If at any time the subject moved and the crosshair was no longer over the eyes of the subject, the experimenter clicked on the crosshairs and repositioned them at the new location over the subject's eyes, and then released it to once again expose the stimulus. If the subject's head panned in a slow arc, scanning in a clockwise or counterclockwise manner indicative of stimulus tracking, the experimenter chose "yes" as a response to the stimulus. This was repeated continually until a visual acuity threshold was reached, above which the subject no longer responded to any stimulus presented and the computer program automatically terminated the test and indicated the individual threshold for each eye.

Contrast sensitivity test administration. Contrast sensitivity thresholds were obtained by using the "contrast sensitivity" program and testing each of 5 spatial frequencies $(0.031,0.064,0.092,0.103,0.192$, and 0.272$)$ independently, as described above. At a given test spatial frequency, the black bars are decreased in intensity until they are indistinguishable as separate from the white bars. The point at which the mouse can no longer see the gradient bars is the contrast sensitivity threshold for that spatial frequency. CS thresholds at each spatial frequency yield a contrast sensitivity function.

Test analysis. All VA data were analyzed in the Minitab express software (Minitab, Inc. State College, PA) by one-way ANOVA using the Fisher's post-hoc analysis. CS functions were compared by two-way ANOVA on the entire CS curve, 
across groups. In the small cohort with pre-blast and post-blast VA data, a pre-post difference was achieved and used for comparison as well

\section{Electroretinogram}

ERGs were performed on anesthetized mice using a Diagnosys Ganzfeld ERG system (Diagnosys, LLC, Lowell, MA). Prior to the test, mice were dark adapted overnight in a light-proof box with attachment to a ventilation hood. On the day of the ERG, they were only exposed to low intensity red lights for the short periods of time required to administer anesthesia and place electrodes.

Test administration. Mice were first weighed, then given an ip injection of ketamine/xylazine (80/10 dilution) at a dose of $0.1 \mathrm{~mL} / 10 \mathrm{~g}$ of body weight. Anesthesia depth was gauged by suppression of tail pinch response. One drop per eye of $1 \%$ cyclopentolate hydrochloride ophthalmic solution USP (Bausch \& Lomb, Tampa, FL) was given to induce pupil dilation, and the mouse was moved to the ERG platform for positioning. The ERG platform had a microwavable heating pad under it to keep the mouse's body temperature from dropping during the test. The mouse's tail and forehead were sanitized with a sterile ethanol swab prior to the insertion of a sub-cutaneous ground and reference electrode, respectively. A drop of methylcellulose gel (Methocel ${ }^{\circledR} 2 \%$, OmniVision, Neuhausen, Swizerland) was placed onto each eye to assist in signal transduction, then left and right gold ring electrodes (Goldring Electrode $2.5 \mathrm{~mm}$, Roland Consult, Havel, Germany) were positioned such that they aligned parallel to the surface of the cornea, and moved closer until they gently touched the surface of the mouse's cornea without exerting undue pressure. Impedance was then measured and corneal electrodes were adjusted to give the lowest possible impedance readings. After this, the ERG flash hood was lowered into place above the mouse such that the mouse was centered in the flash zone (video image guided). At this point, all red lights were turned off, and the test was started. If at any point during the test, an electrode began to flat line, the test was paused, and resumed once the electrode background noise became visible again. This occurred when large transient impedance spikes forced the electrode to go offline, and happened variously throughout the test, likely because of body movement from the mouse breathing. At the end of the test, all electrodes were removed and sanitized, and the mouse was placed on a heating pad for recovery. Their eyes were rinsed with sterile saline to prevent irritation, and they were placed back in their cages with their littermates once they began ambulation.

Test analysis. Espion software (Version 5.0.52, Diagnosys, LLC) was used to analyze ERG data. At each light intensity tested (step), anywhere from 10-20 waveforms were captured during ERG administration. The number captured was determined by the intensity of light, with lower intensities having more captures, and higher intensities having fewer captures (to prevent photo bleaching). Anomalous or flat-line waveforms were removed prior to averaging of the waveforms at each intensity. Anomalous waveforms were defined as having an opposite direction from the normal trend of the ERG wave, and/or having large, regular spike and wave patterns indicative of 
electromagnetic interference (for example, $60 \mathrm{~Hz}$ waveform seen with nearby electronics plugged into a wall outlet). Measurements of peak A- and B-wave amplitude, as well as latency time, were taken from the averaged waveform at each light intensity. This analysis was performed on every pre- and post-blast test for each subject.

To analyze the ERG results, pre-blast test results were compared with post-blast test results within the same animal to achieve a $\Delta$-value that was then used for statistical purposes. Subsequently, control pre-test averages were used at each step and were added to each individual $\Delta$-value to achieve a scaled ERG peak value that was easier to conceptualize for graphing purposes.

\section{Results}

For the purpose of discussing the three groups of animals used in these experiments, the sham-blasted, vehicle treated group will be referred to as $0 \mathrm{~V}$, the $50 \mathrm{psi}$ blasted, vehicle-treated group will be referred to as $50 \mathrm{~V}$, and the 50 psi-blasted, drug treated group will be referred to as 50SMM for the remainder of this dissertation.

\section{OKN}

Visual acuity. Visual acuity thresholds from all groups were compared using one-way ANOVA and Fisher's individual tests for differences in means. Left eye thresholds were not significantly different between any group by either metric (ANOVA $\mathrm{p}=0.8275$ ). Similarly, right eye VA thresholds were not significantly different between any group by either metric (ANOVA $\mathrm{p}=0.4654$ ). Furthermore, the post-blast VA data for a small group of animals was compared to their available pre-blast VA data, and the preblast versus post-blast difference was found to be insignificant for both left and right eye $(\mathrm{p}=0.3591$ and $\mathrm{p}=0.5024$, respectively) $($ Figure $4-1)$.

VA analysis in long term multi-blast animals at a year from blast found significant differences in both eyes between the 0 - and 50-psi groups. 0-psi LE VA was significantly different than the 50-psi group by one-way ANOVA ( $\mathrm{p}=0.0001)$. In the RE, the 50-psi group was also significantly different from the 0 -psi group by one-way ANOVA $(\mathrm{p}=0.0463)$ (Figure 4-2).

Contrast sensitivity. Contrast sensitivity functions were compared using a twoway ANOVA across all spatial frequencies and groups. In the left eye, significant differences were found between both the sham group and the $50 \mathrm{~V}$ group $(\mathrm{p}=0.0041)$, and the drug treated 50-psi group and the $50 \mathrm{~V}$ group $(\mathrm{p}=0.0202)$, while the the sham and drug treated blast group had no significant difference between them $(\mathrm{p}=0.4696)$ (Figure 4-3). In the right eye, even more robust differences were seen between the sham group and $50 \mathrm{~V}$ group $(\mathrm{p}<0.0001)$ and the drug treated blast group and the $50 \mathrm{~V}$ group $(\mathrm{p}<0.0001)$. No difference existed in the right eye between the sham and the drug treated blast group 


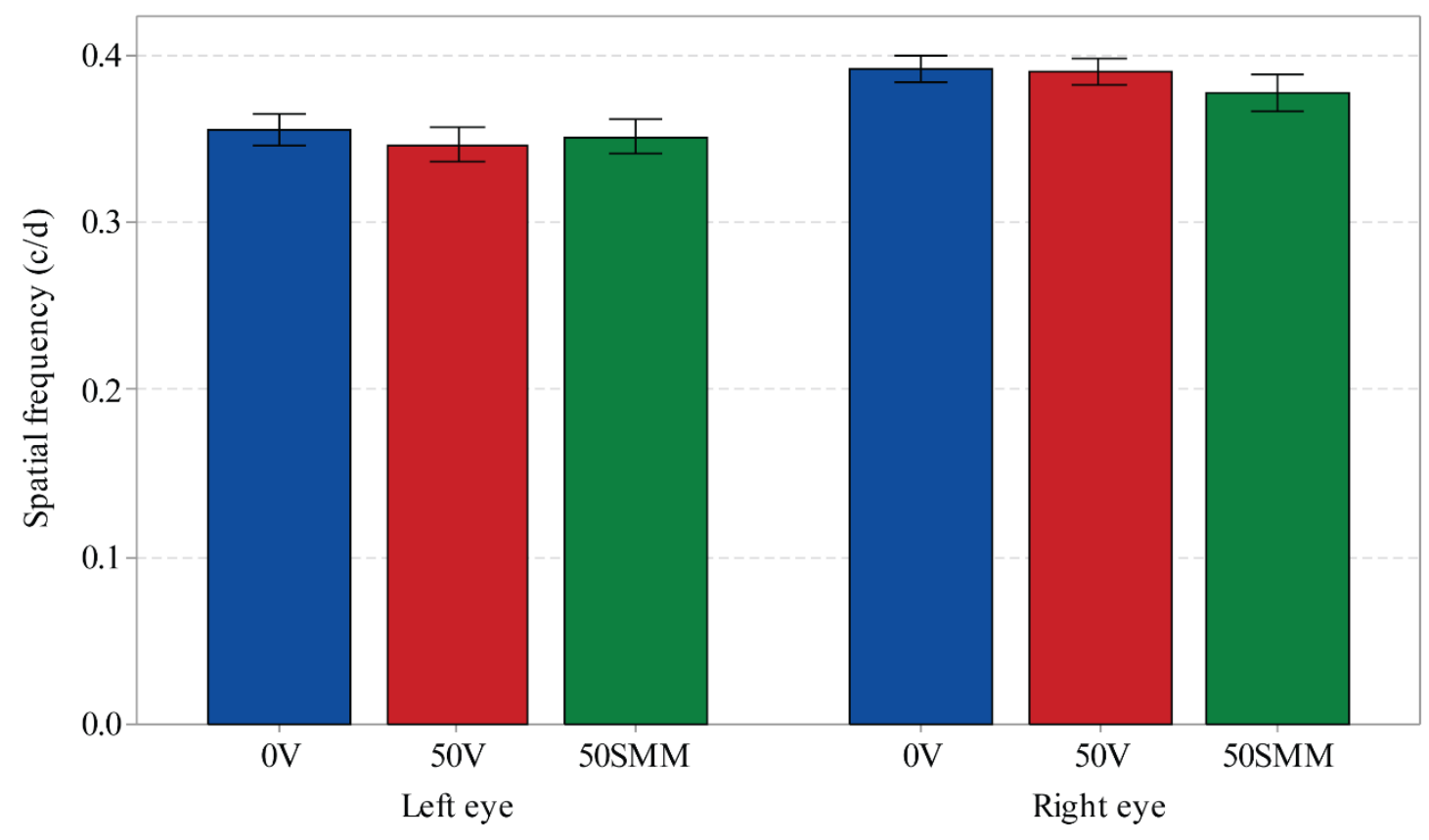

Figure 4-1. Visual acuity thresholds for both eyes at 30 days after blast.

There were no significant changes in the VA thresholds of either eye for any group. Bars are one standard error from the mean. 


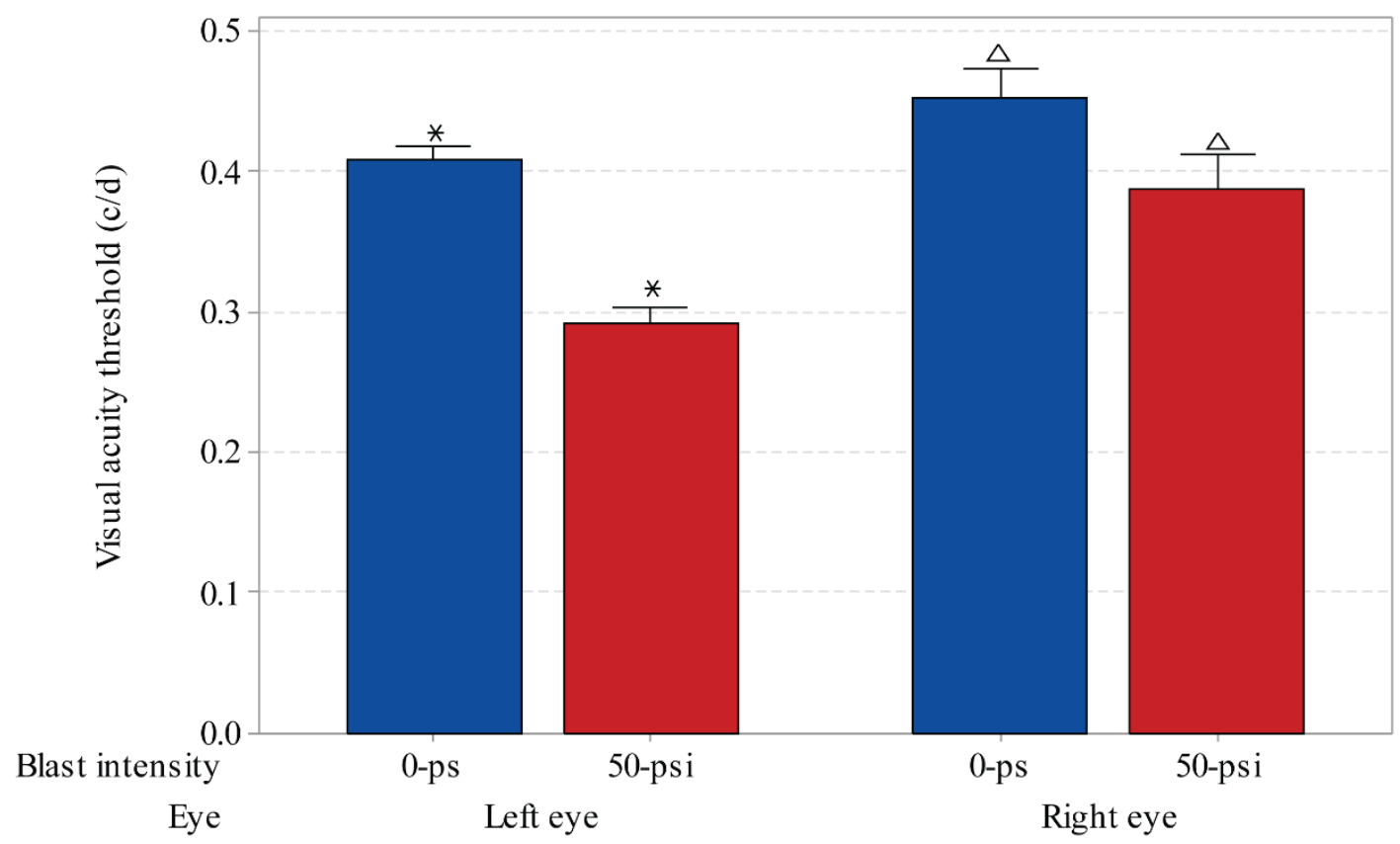

Figure 4-2. Visual acuity thresholds for $4 x$ multi-blast animals a year after blast.

Visual acuity in 50-psi animals is significantly reduced in both eyes compared with controls. Asterisk denotes significance at $p=0.0001$. Triangle denotes significance at $\mathrm{p}=0.0463$. Bars are one standard error from the mean. 


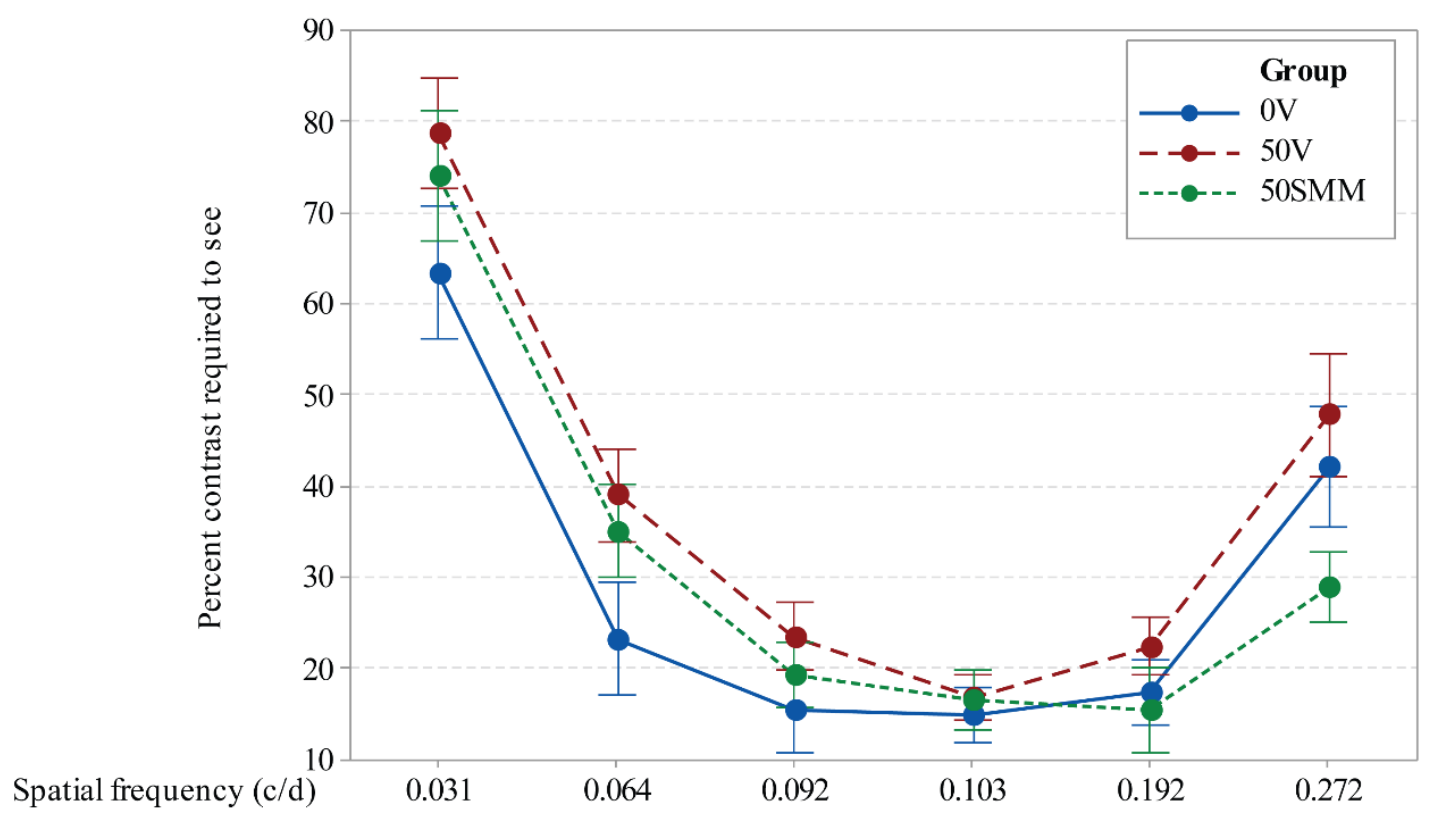

Figure 4-3. Contrast sensitivity functions of the left eye at 30 days after blast.

In the left eye, there was a significant increase in the contrast sensitivity function of $50 \mathrm{~V}$ animals (red), indicating a need for higher levels of contrast across all spatial frequencies in order to perceive a given stimulus. This was significantly, but not completely, remedied by SMM-189 treatment (green). Bars are one standard error from the mean. 
$(\mathrm{p}=0.2938)$ (Figure 4-4). These results show that contrast sensitivity was reduced after 50-psi blast, and the deficit was rescued with SMM-189.

In the long-term multi-blast animals, CS was measured at only one spatial frequency, $0.272 \mathrm{c} / \mathrm{d}$. The 50-psi animals were found to have a significant CS increase in the left eye over 0 -psi animals by one-way ANOVA ( $\mathrm{p}=0.0277)$. In the right eye, 50-psi animals were also significantly increased over 0-psi animals $(\mathrm{p}=0.0345)$ (Figure 4-5).

\section{ERG}

Left eye. A-wave peak amplitudes and latency time were unchanged across all three groups by two-way ANOVA ( $\mathrm{p}=0.3233$ for peak amplitude and $\mathrm{p}=0.8177$ for latency time) (Figure 4-6C). Pre-blast and post-blast B-wave peak amplitude measurements were unchanged in $0 \mathrm{~V}$ and $50 \mathrm{SMM}$ groups by a paired t-test $(\mathrm{p}=0.3243$, and $\mathrm{p}=0.4250$, respectively), however, the $50 \mathrm{~V}$ group did significantly differ from their pre-blast measurements $(\mathrm{p}=0.0455)$ (Figure 4-7). Furthermore, two-way ANOVA showed the B-wave peak amplitudes to be significantly increased in $50 \mathrm{~V}$ animals when compared to controls $(\mathrm{p}=0.0379$ ), while 50-psi SMM-treated animals were not significantly different from either vehicle-treated blast animals $(\mathrm{p}=0.2222)$ or vehicletreated control animals $(\mathrm{p}=0.5128)$ (Figure 4-6A). B-wave latency time was statistically unchanged across all groups $(\mathrm{p}=0.2637)$ (data not shown). These results show that the $\mathrm{B}$ wave was abnormally elevated after 50-psi blast, and the abnormality was rescued with SMM-189.

Right eye. A-wave peak amplitude and latency time were unchanged across all groups by two-way ANOVA ( $p=0.2660$ and $p=0.7036$, respectively) (Figure 4-6D). Bwave peak amplitude and latency time were unchanged across all groups by two-way ANOVA ( $\mathrm{p}=0.2203$ and $\mathrm{p}=0.8192$, respectively) $($ Figure 4-6B).

\section{Summary}

Functionally, blasted, vehicle-treated mice show mild vision impairment that is slightly different in each eye. The left eye of blasted animals shows a defective ERG response specifically in the part of the ERG wave that corresponds to cells in the inner nuclear layer, a retinal layer where previous studies have shown thinning in these animals. Oddly, the ERG defect is an increase in peak amplitude, rather than the expected decrease under pathologic conditions. The drug-treated animals had a decrease toward normal, with an ERG that was statistically unchanged from control animals. The visual acuity of blasted, vehicle-treated mice was normal in both eyes, and statistically unchanged from both pre-blast/post-blast comparisons in the same individual, and when compared to control animals. However, the contrast sensitivity function showed significant defects in both eyes. Surprisingly, the right eye CS deficit was more robust than that of the left eye, even when the right eye ERG wave was apparently normal. This hints that there may be some different mechanism underlying the pathology of the right 


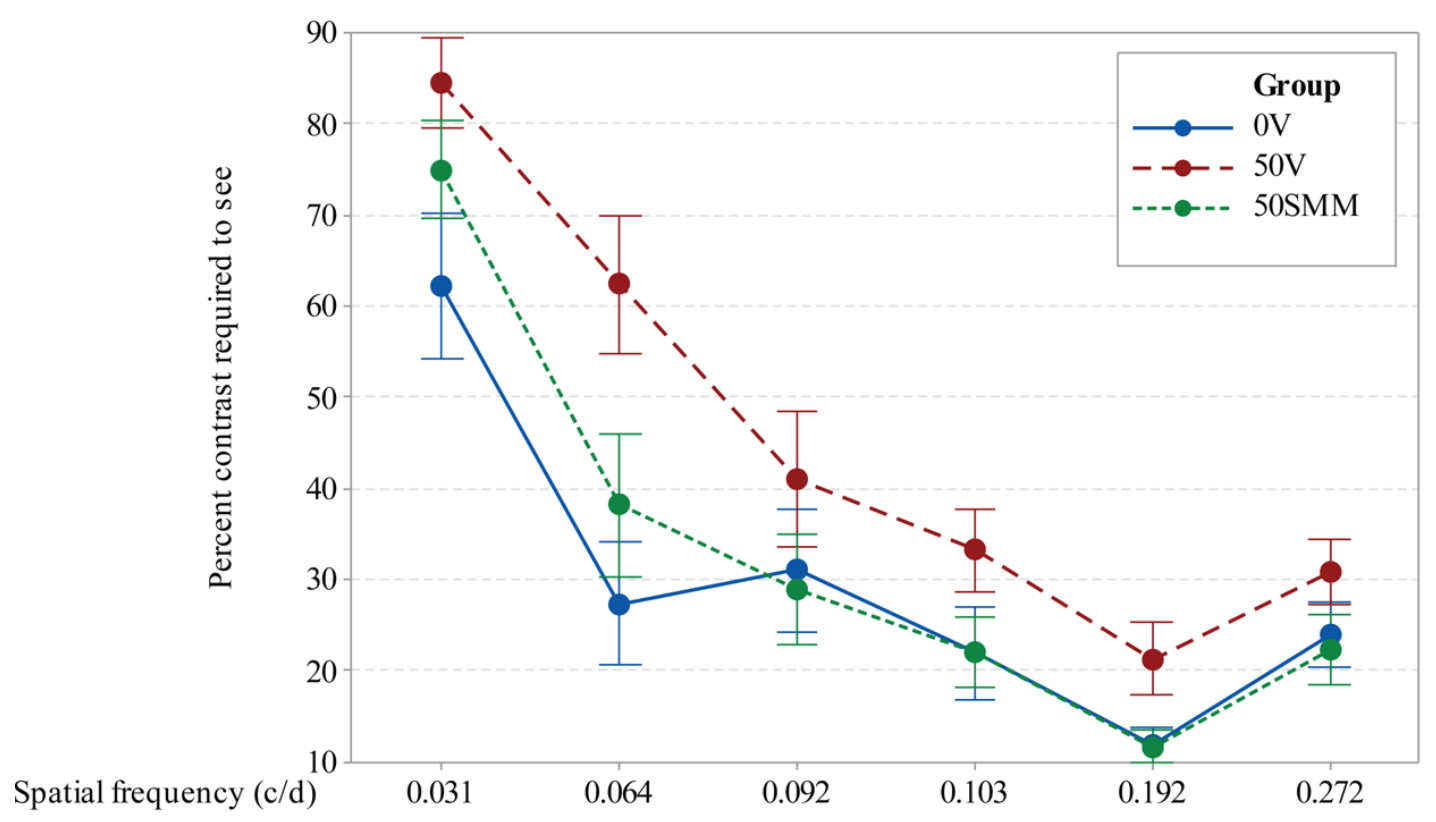

Figure 4-4. Contrast sensitivity functions of the right eye at 30 days after blast.

The right eye of $50 \mathrm{~V}$ animals showed a very significant deficit in contrast discrimination across all spatial frequencies compared to $0 \mathrm{~V}$ animals (red and blue, respectively). This was almost completely remedied with SMM-189 treatment (green). Bars are one standard error from the mean. 


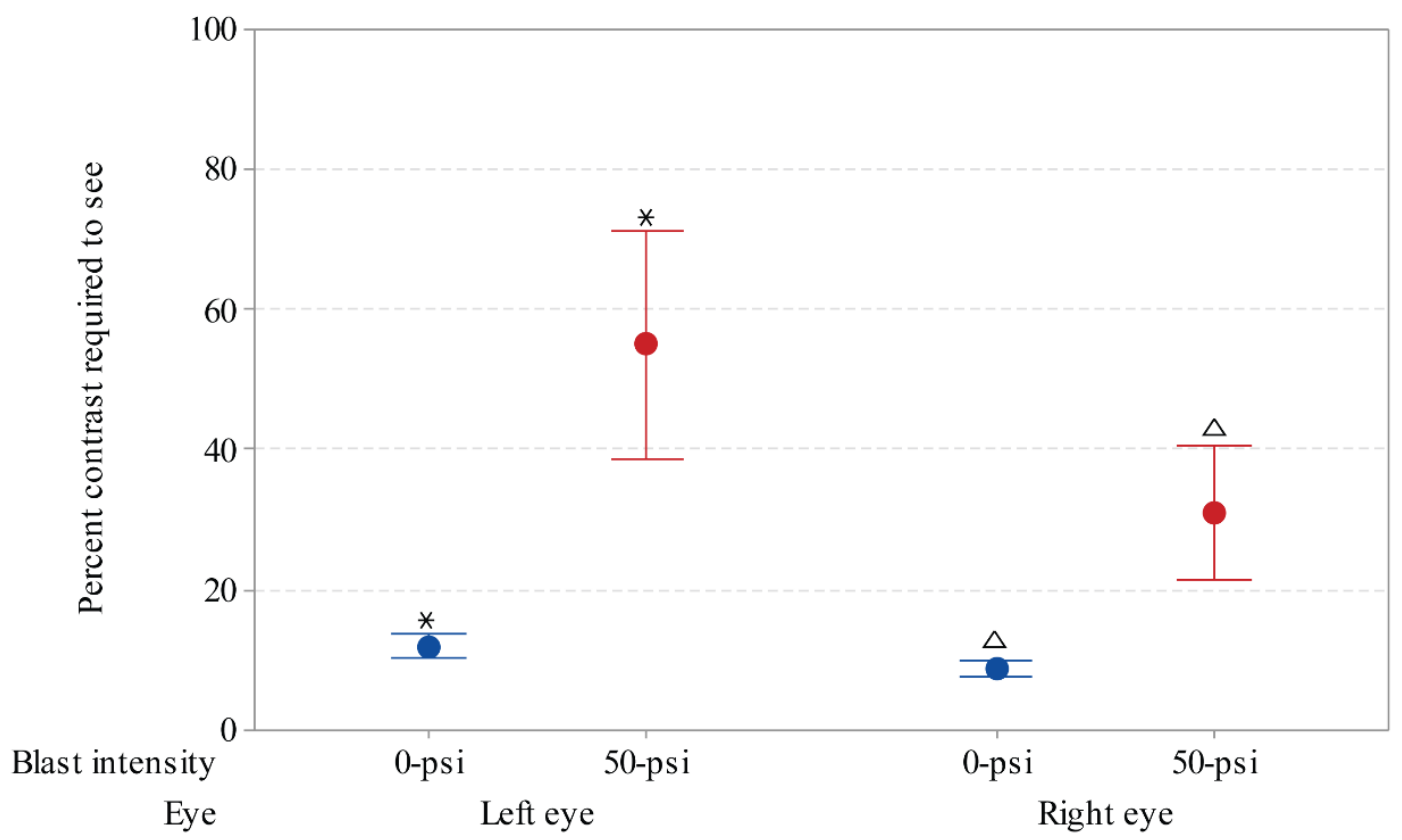

Figure 4-5. Contrast sensitivity in $4 x$ multi-blast animals a year from blast.

Contrast sensitivity at $0.272 \mathrm{c} / \mathrm{d}$ was pathologically increased in 50-psi animals compared to 0-psi animals in both left and right eyes. Asterisk denotes significance at $\mathrm{p}=0.0277$. Triangle denotes significance at $p=0.0345$. Bars are one standard error from the mean. 
A

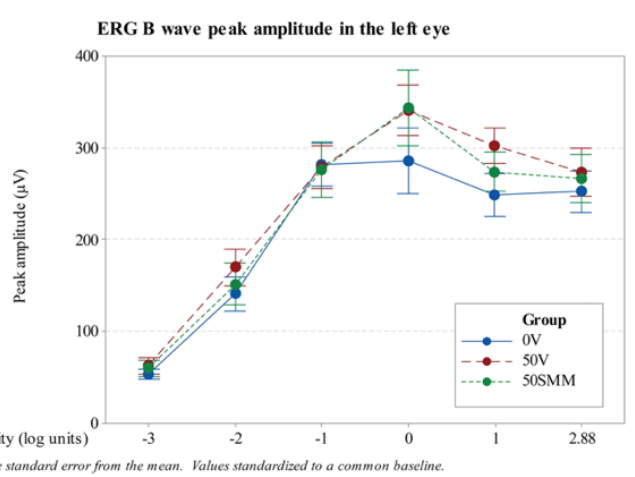

C

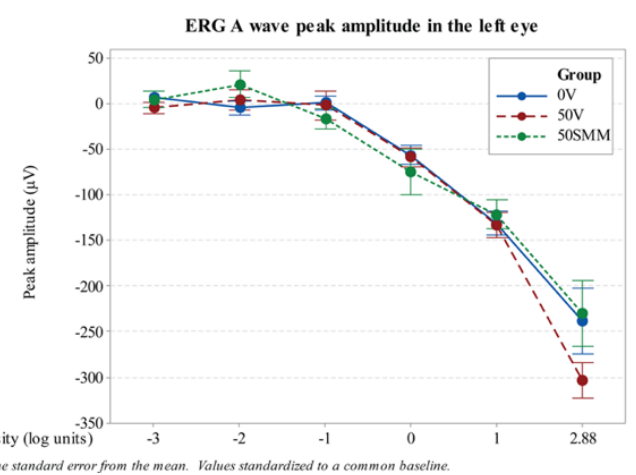

B

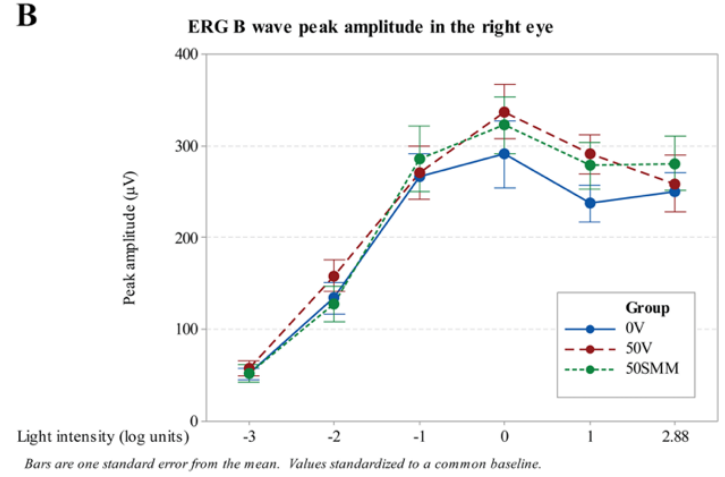

D

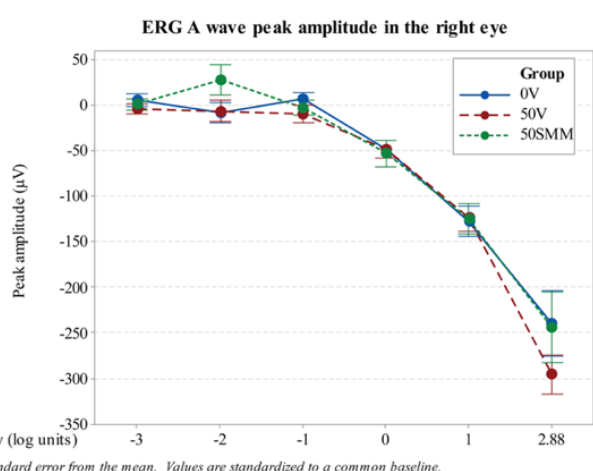

Figure 4-6. The effect of blast and SMM-189 treatment on ERG A- and B-wave functions at 30 days after blast.

This figure shows the average scotopic ERG peak amplitudes at 6 light intensities for all groups. In the left eye of $50 \mathrm{~V}$ animals, the B-wave is significantly increased across all intensity levels (A), while the right eye is nearly so (B). Drug treatment slightly rescues this anomalous increase in both eyes, being statistically equivalent with controls. The Awaves of the $50 \mathrm{~V}$ left eyes are nearly different from that of controls $(\mathrm{C})$, while the right eyes of all groups are statistically equivalent (D). 
A
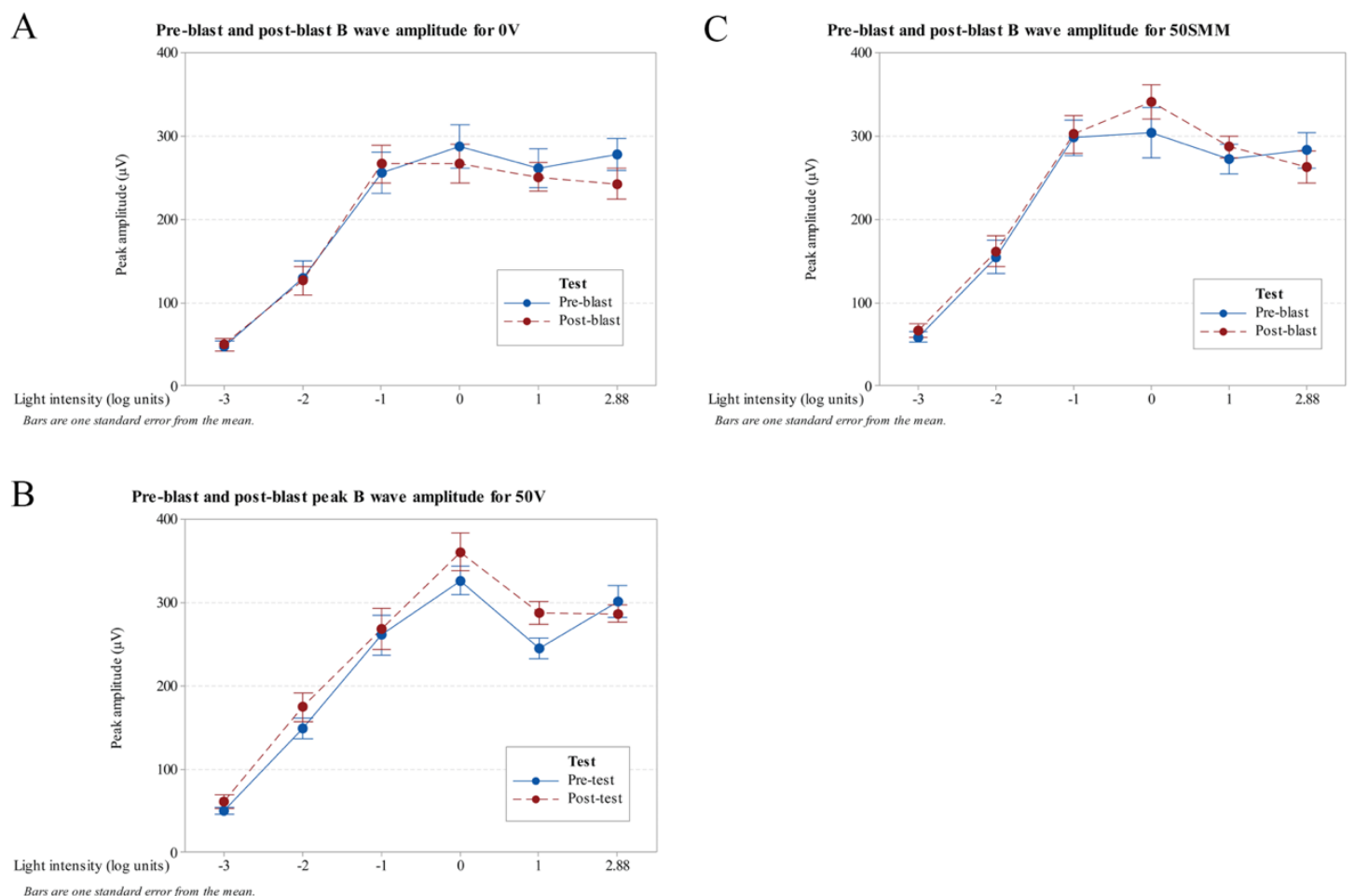

Figure 4-7. Left eye pre-blast and 30 days post-blast scotopic ERG B-wave functions for all groups.

Left eye post-blast ERG functions were significantly elevated in the $50 \mathrm{~V}$ group from their pre-blast values (B), but not in the $0 \mathrm{~V}(\mathrm{~A})$ or 50SMM groups $(\mathrm{C})$. 
eye than the left eye. Visual acuity in multi-blast animals at a year from blast showed significant deficits in the left eye of 50-psi animals. The right eye visual acuity was only significantly compromised in 50-psi animals, indicating again that there seems to be a difference in the mechanism of LE and RE damage. Contrast sensitivity was also pathologically elevated in the 50-psi animals a year after $4 x$ blast in both eyes. These multi-blast findings confirm the persistence of visual function pathology at an extended time point after blast. 


\title{
CHAPTER 5. STRUCTURAL VISION OUTCOMES IN MTBI
}

\author{
Methods
}

\section{Optical coherence tomography (OCT)}

Test administration. A Bioptigen 840nm SDOCT system (Bioptigen Inc., Morrisville, NC) with Superlum Broadlighter at a $150 \mathrm{~nm}$ bandwidth was used for obtaining $\mathrm{a}<3.5 \mu \mathrm{m}$ resolution image of the retina in live, anesthetized mice. Mice were first weighed and injected with ketamine/xylazine (80/10 dilution) dosed at $0.1 \mathrm{~mL} / 10 \mathrm{~g}$ of body weight, then placed on a heating pad to maintain body heat. Anesthesia depth was gauged by suppression of tail pinch response. Once fully anesthetized, one drop of $1 \%$ cyclopentolate hydrochloride ophthalmic solution USP (Bausch \& Lomb, Tampa, FL) was placed into each eye to attain pupil dilation. Mice were then placed into the mouse alignment holder and maneuvered into position. One drop of Systane ${ }^{\mathrm{TM}}$ (Alcon, Fort Worth, TX) eye drops were placed into the eye directly before imaging began, to assist in achieving good image resolution as well as to keep the eye from drying out. Mouse eyes were positioned such that the central retinal artery was fully visible on fundoscopic view and approximately mid-center, and one to three images were obtained from each eye, depending on image quality achieved. Mice were then removed, and a sterile $.9 \%$ saline solution was used to wash the eyes and prevent irritation. Mice recovered on a heating pad and were placed with littermates upon recovery of ambulation.

Test analysis. OCT images were loaded into the InVivoVue software (Bioptigen Inc., Morrisville, NC), which was used to perform measurements of retinal layers. The following measurements were made: total retina, inner plexiform layer, inner nuclear layer, outer nuclear layer, and photoreceptor segments (inner and outer segments measured together). These measurements were made at the horizontal meridian in sections where the optic nerve head and/or central retinal artery were clearly visible. The measurements were performed $50 \mu \mathrm{m}$ from the optic nerve head on both the nasal and temporal sides of each eye, and then averaged. Post-blast measurements were subtracted from pre-blast measurements for each animal in both eyes and at each layer. This yielded a $\Delta$-value that was then used for statistical purposes.

\section{Histologic processing}

Sectioning. Fixed brains were first frozen with dry ice, then sectioned on a sliding microtome in the transverse plane at $35 \mu \mathrm{m}$. Each brain was collected as 12 separate series in $0.1 \mathrm{M}$ PB with $0.02 \%$ sodium azide.

Prior to sectioning, eyes were placed into a 50/50 Optimum Cutting Temperature compound (O.C.T.) (Tissue-Tek®, Sakura Finetek USA, Inc.)/ 20\% sucrose and $0.1 \mathrm{M}$ 
PB solution for 10 minutes in a vacuum chamber at 16 psi. Then they were moved into $100 \%$ O.C.T. and left to equilibrate at ambient pressure for 30 minutes. Afterward, they were once again placed in the vacuum chamber at 16 psi for 10 minutes. This was to enable the O.C.T. medium to completely penetrate the tissue, and to remove any air bubbles prior to freezing. The eyes were then oriented in the mold to allow sectioning of the eyes through the horizontal meridian and optic nerve/optic nerve head. The microsutures were used to maintain nasal-temporal orientation throughout the embedding procedure, and the eyes were finally frozen on dry ice inside the molds. When fully frozen, the embedded eye block was removed from the mold, and eyes were sectioned on the cryostat (Bright Instrument Company, Ltd., Huntingdon, England) at $-16^{\circ} \mathrm{C}$ at thickness of $20 \mu \mathrm{m}$. Sections were discarded until the full retina was visible in the section, approximately $100 \mu \mathrm{m}$ into the eye, and then each section was collected onto a Superfrost ${ }^{\circledR} /$ Plus slide (Fisher Scientific, Pittsburg, PA), then placed onto a slide warmer at $32{ }^{\circ} \mathrm{C}$ for 5 minutes, before being transferred to the freezer $\left(-80^{\circ} \mathrm{C}\right)$ for storage prior to immunohistochemistry (IHC) processing.

Staining and immunohistochemistry. All immunohistochemistry was performed on free-floating sections (brain), or slide-mounted sections (eyes) in a standardized manner that involved the following general steps: (1) 3 5-minute washes in $\mathrm{PB}(\mathrm{pH}=7.4)$, then (2) incubation in primary antibody solution at room temperature for 24 hours, then (3) 3 5-minute washes in PB, then (4) incubation in secondary antibody solution at room temperature for 2 hours, and finally (5) 3 5-minute washes in PB before mounting (brains), drying, and cover-slipping. See Table 5-1 for a list of all primary antibodies and dilutions used for these experiments.

The primary antibody solution always consisted of the primary antibody/antibodies diluted into a solution of 5\% normal horse serum (NHS) and $0.8 \%$ Triton X-100/0.01\% sodium azide/0.1M PB (PBX). Free-floating sections were incubated in micro-centrifuge tubes with $500 \mu \mathrm{L}$ of primary antibody solution on a countertop rotator, while slide-mounted tissue (eyes) were first outlined with hydrophobic pen (ImmEdge, Vector Labs: Burlingame, CA), then incubated with $250 \mu \mathrm{L}$ of primary antibody solution per slide and kept stationary. Secondary antibody solutions always consisted of the secondary antibody/antibodies diluted into PBX without sodium azide, and were shielded from light during the 2-hour incubation period, since fluorescently-labeled secondary antibodies were used in all IHC studies. All slides used for IHC were cover-slipped with Vectashield Antifade mounting medium with DAPI (Vector Labs: Burlingame, CA). The edges of coverslipped slides were painted with nail polish (Sally Hansen, NYC) to prevent leakage of the mounting medium during imaging.

Optic nerves used for axon quantifications were embedded in plastic, sectioned transversely at $1 \mu \mathrm{m}$, and then stained with $1 \%$ p-phenylenediamine in $50 \%$ methanol (PPD) as described in Bricker-Anthony et al ${ }^{177}$. 
Table 5-1. Primary antibodies used for immunohistochemistry.

\begin{tabular}{llll}
\hline Antibody target & Host & Dilution & Source \\
\hline GFAP & Rabbit & $1: 100$ & Immunostar \\
Brn3a & Goat & $1: 100$ & $\begin{array}{l}\text { Santa Cruz } \\
\text { biotechnology }{ }^{\circledR}, \text { Inc. }\end{array}$ \\
Iba-1 & Rabbit & $1: 500$ & $\begin{array}{l}\text { Wako Chemicals } \\
\text { USA, Inc. }\end{array}$ \\
CD16/32 & Rat & $1: 50$ & Abcam ${ }^{2}$ \\
CD206 & Goat & $1: 200$ & R\&D Systems ${ }^{\circledR}$, Inc. \\
\hline
\end{tabular}




\section{Imaging}

Light microscopy. For optic nerve axon quantification, images were captured using an Olympus BH2 series light microscope with S Plan Apochromat objectives, an achromatic condenser (Olympus Corporation, Tokyo, Japan) and SPOT idea TM camera (Diagnostic instruments, Inc., Sterling Heights, MI) running on SPOT Advanced software (Version 4.6). Lens magnification of 20x was used to capture images for axon cross section measurements. Axon counts were done on sections at 100x oil immersion. A scale bar was also imaged at each photo session to standardize size comparisons across magnifications.

Confocal laser scanning microscopy. A Zeiss 710 CLSM microscope (LSM 800, Carl Zeiss AG, Oberkochen, Germany) was used to image tissue in IHC studies for this dissertation. Laser power, gain, and wavelength were adjusted to optimize image quality, and were standardized across all images for a given marker (or combination of markers). Retinal images were always obtained on the horizontal meridian at the level of the optic nerve head when possible, and from the nasal and temporal side of the eye. Images were always obtained at 20x power, with multiple images stitched together using the tile capture feature of the Zen software (Zen Black Version 2.1, Carl Zeiss AG, Oberkochen, Germany) in order to quantify large lengths of retina in a single image.

\section{Quantification and analysis of structural end points}

For all images of a given marker, contrast, brightness, and color values were adjusted in a standard way across every image, in order to optimize viewing for quantification. All analysis was done in a blinded manner. Unless otherwise noted, ImageJ software (Version 1.50c4, National Institutes of Health, USA) and the Analyze $>$ Cell counter plugin were utilized for quantification of histology endpoints, from images obtained by either LM or CLSM. Measurements of histology endpoints were scaled to the correct image magnification using scale bars either obtained directly (LM) from scale bar photographs at a known magnification, or indirectly (CLSM) from the image software itself.

Axons in the optic nerve (LM). Whole optic nerves were measured for crosssectional area using the Image J freehand selection tool and Analyze $>$ Measure command. Then, high power images were examined from each of 4 quadrants (north, south, east, west) of the optic nerve cross section for each eye. For axon quantification, an image from a single quadrant was overlaid with a $10 \times 10 \mu \mathrm{m}$ grid using the Analyze $>$ Grid tool. Starting from the top row, a random number generator was used to determine which box in each row would be quantified. As an example, in a row of 10 grid boxes, the random number generator was set to populate a number between 1 and 10 . If the number generated was 8 , then the box $8^{\text {th }}$ from the left side of the image would be the box in which axons were counted using the cell counter tool. At each quantification box, axons falling on the north and east line of the grid were counted, whereas axons falling on the south and west line of the grid were not counted. Axons were only counted if a well- 
defined dark ring surrounding a lighter center area could be seen. Thus, axons too small to have an apparent central clearing (axoplasm) were likely missed, due to the resolution/magnification restrictions of light microscopy.

Once an entire image was counted, the total number of axons was divided by the total box area counted (each box was a known size, $10 \times 10 \mu \mathrm{m}$, and the number of boxes quantified was the total number of rows, since one box was counted per row, thus the total area in which axons were counted was $100 \mu \mathrm{m}^{2} \mathrm{x}$ number of rows) in order to give an axon density for that quadrant. Finally, the 4 quadrants for that $\mathrm{ON}$ were averaged, and total $\mathrm{ON}$ axon number was extrapolated using the axon cross sectional area and the axon density for that $\mathrm{ON}$ (axon density $\mathrm{x}$ axon area). For statistics purposes, total axon number, ON cross sectional area, and axon density were all compared for each optic nerve and each group.

Brn3a+ cells in the retina (CLSM). Retinal images from the nasal and temporal side of each eye were used to quantify RGCs using an antibody to Brn3a. Brn3a is visible in the cell body of cells, and cells were only counted if they were clearly visible in the section, with a nucleus visible on the overlaid DAPI channel. Brn3a-positive cells were counted, and the total length of the retina over which they were counted was measured. This then yielded a number of cells per $\mu \mathrm{m}$ length of retina for each side of the eye, which were then averaged together. The average number of cells per $\mu \mathrm{m}$ length of retina for each eye were used for comparison and statistics purposes.

Iba-1+ cells in the retina (CLSM). Iba-1+ cells (microglia) were quantified in a manner similar to Brn3a+ cells previously described. The exception is that Iba- 1 is also visible in the processes of microglia, so many more processes were visible than cell bodies. Cells were only counted if the cell body was clearly visible in the section, and the DAPI channel showed a clear nucleus. Microglial processes were not quantified. Average number of cells per $\mu \mathrm{m}$ length of retina for each eye were used for comparison and statistics purposes.

GFAP reactive processes in the retina (CLSM). As previously, retinas were quantified on the nasal and temporal side for each eye. GFAP+ processes normally exist on the deep surface of the retina, in the nerve fiber layer. Only processes that extended past the RGC layer and were clearly visible were quantified. The total length of the retina over which GFAP processes were counted was measured, and this was used to yield a number of processes per $\mu \mathrm{m}$ length of retina. As before, the nasal and temporal sides were averaged together, and this number of processes per $\mu \mathrm{m}$ length was used for comparison and statistics purposes.

M1/M2 cells in the right optic tract (CLSM). Recall that the major damage in the white matter seen in this model originates from the left eye, where the axons in the left optic nerve almost completely decussate in the optic chiasm to continue in the brain as the right optic tract. To simplify the experimental design and focus solely on a region of known injury, images of the right optic tract were captured using a $4 \mu \mathrm{m}$ thick z-stack, capturing separate channels for CD16/32, CD206, and Iba-1. The maximum intensity 
projection of the z-stack was used for analysis purposes. In order to quantify cellular staining for both M1 and M2 markers, we used a method described by McCloy and colleagues that involves calculating a corrected cellular fluorescence level for individual cells of interest ${ }^{220}$. Using ImageJ, individual microglial cells in the optic tract were circumscribed using the free-form drawing tool on the Iba- 1 channel. With this outline in place, the channel was switched to visualize CD16/32 and CD206 independently, where measurements of the staining intensity of each channel were collected using the integrated density values. This was repeated for every visible cell in the optic tract. Afterward, 5 measurements were taken randomly throughout the optic tract over areas where no Iba-1 staining was visible, and the previously described process was repeated. These values were averaged together to represent the background staining intensity for each channel.

In order to determine the corrected cellular fluorescence, the background staining was multiplied by the area of the cell, and this was subtracted from the integrated density value of that cell. This value, the corrected cellular fluorescence, was then used for direct comparison. For each cell, a ratio of M1 (CD16/32) and M2 (CD206) markers were obtained, and from this number a cell was then categorized as M1-biased (an M1/M2 ratio equal to or above 1.10), M2-biased (M1/M2 ratio equal to or below 0.90), or nondifferentiated (M1/M2 ratio between 1.09 and 0.91 ). The categorical distinction of M1, M2, or M0 for each cell was used for statistical analysis.

Axon bulbs in the optic tract (CLSM and LM). Axon bulbs are clearly visible in the optic tract at 3 days post-blast, as described previously. However, at 7 days, the bulbs become smaller and more granular in appearance, at this point they are likely indicative of axon degeneration fragments. Axon bulbs were visualized by either SMI-32 immunolabeling or direct EYFP immunofluorescence in reporter mice (described previously), and were counted in tissue from animals 3, 5, and 7 days after blast. Some of the tissue used for axon bulb quantification at 5 and 7 days after blast was in animals exposed to 60 psi blast, and for the purposes of this analysis, was grouped together with animals who received 50 psi blasts. Images were captured by either CLSM or LM. Within ImageJ, the freehand tool was used to outline the entire ROT region, and the area measurement was acquired. Then, axon bulbs whose longest dimension was greater than $6.5 \mu \mathrm{m}$ were counted. The axon bulb areal density was calculated by dividing the number of bulbs by the area of the ROT section. These axon bulb densities were used for direct statistical comparison.

\section{Results}

\section{OCT}

Pre-blast and post-blast measurements for each animal were compared using a paired t-test. In 50V animals, the left eye showed significant thinning in the outer retina $(\mathrm{ONL}+$ photoreceptor segments $)$ from their pre-blast measurements $(\mathrm{p}=0.0076)$. The 
inner retina (IPL $+\mathrm{INL})$ showed no significant changes in the $50 \mathrm{~V}$ animals $(\mathrm{p}=0.6238)$, but did show significant thinning in the 50SMM animals $(\mathrm{p}=0.0427)$ (Figure 5-1). In the right eye, no significant changes from pre to post-blast measurements were seen in any group (Figure 5-2).

\section{Optic nerve}

Left eye. The ON cross sectional area was significantly lessened in both the $50 \mathrm{~V}$ and 50SMM animals when compared with $0 \mathrm{~V}$ animals by one-way ANOVA ( $\mathrm{p}=0.0393$ and $\mathrm{p}=0.028$, respectively) (Figure 5-3). In $50 \mathrm{~V}$ animals, the axon density (count per $\mu \mathrm{m}^{2}$ ) was significantly less than in the $0 \mathrm{~V}$ group by one-way ANOVA $(\mathrm{p}=0.0198)$, but not significantly different than the 50SMM group $(\mathrm{p}=0.1512)$ (Figure 5-4). The $0 \mathrm{~V}$ and 50SMM groups were not significantly different in axon density. The extrapolated total $\mathrm{ON}$ axon count was significantly decreased in $50 \mathrm{~V}$ animals compared to $0 \mathrm{~V}$ animals $(\mathrm{p}=0.0014)$, as well as in 50SMM animals when compared to $0 \mathrm{~V}$ animals ( $\mathrm{p}=0.0136$ ), and the $50 \mathrm{~V}$ and $50 \mathrm{SMM}$ groups were not significantly different from one another in extrapolated total $\mathrm{ON}$ axon count (Figure 5-5). A Pearson correlation revealed a strong negative relationship between the axon density and $\mathrm{ON}$ area in control animals that was significant $(\mathrm{r}=-0.7138, \mathrm{p}=0.0136) .50 \mathrm{~V}$ animals a weak positive relationship that was not significant $(\mathrm{r}=0.1331, \mathrm{p}=0.7140)$, and 50SMM animals showed a weak positive correlation that was also not significant $(\mathrm{r}=0.2453, \mathrm{p}=0.4946)$.

Right eye. The ON cross sectional area was not significantly changed between any group, however the axon density was significantly decreased in $50 \mathrm{~V}$ animals when compared with both $0 \mathrm{~V}$ and 50SMM animals by one-way ANOVA ( $\mathrm{p}=0.0002$ and $\mathrm{p}=0.0438$, respectively) (Figures 5-3 and 5-4). The $0 \mathrm{~V}$ and 50SMM animals were nearly, but not statistically different in axon density $(\mathrm{p}=0.0571)$. The extrapolated total $\mathrm{ON}$ axon count was not significantly different between any group, although the $50 \mathrm{~V}$ were nearly significantly different from $0 \mathrm{~V}$ group at $\mathrm{p}=0.0639$ (Figure 5-5). A Pearson correlation revealed a moderate negative correlation in controls that was not significant $(\mathrm{r}=-0.2977, \mathrm{p}=0.3909), 50 \mathrm{~V}$ animals showed a strong negative relationship that was nearly significant $(\mathrm{r}=-0.5554, \mathrm{p}=0.0761)$, and 50SMM animals showed a weak negative relationship that was not significant $(\mathrm{r}=-0.2415, \mathrm{p}=0.5015)$.

\section{Brn3a counts}

At 30 days after blast, the left eye Brn3a cell counts were not significantly different between any group using one-way ANOVA. The difference between the sham and $50 \mathrm{~V}$ group was, however, nearly significant $(\mathrm{p}=0.0507)$. In the right eye, all groups were not significantly different from one another (Figure 5-6). 


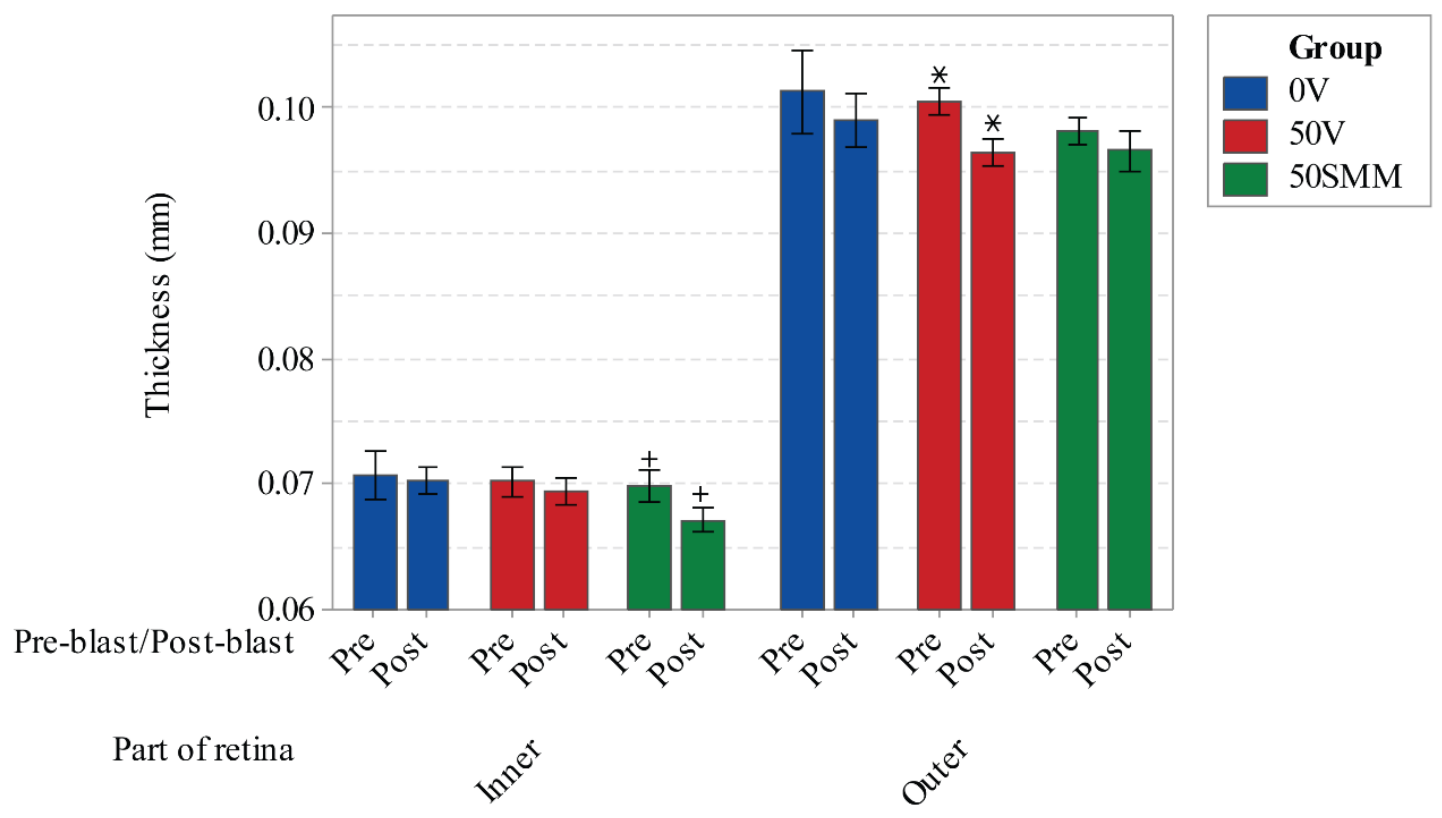

Figure 5-1. Left eye pre-blast and post-blast OCT measurements.

The pre-blast and post-blast OCT measurements for all groups are shown in this figure, grouped by inner and outer retinal layers. Inner retinal layers (IPL+INL) were not significantly changed in $0 \mathrm{~V}$ or $50 \mathrm{~V}$ animals, however drug treatment caused an odd decrease in the inner retinal thickness (denoted by,$+ p=0.0427$ ). The $50 \mathrm{~V}$ animals showed a significant decrease in the outer retina (ONL+photoreceptor layers) after blast (denoted by $*, p=0.0076$ ), while the $0 \mathrm{~V}$ and 50SMM animals showed no significant changes. The total retinal thickness was not changed from pre-blast to post-blast in any group (data not shown). Bars are one standard error from the mean. 


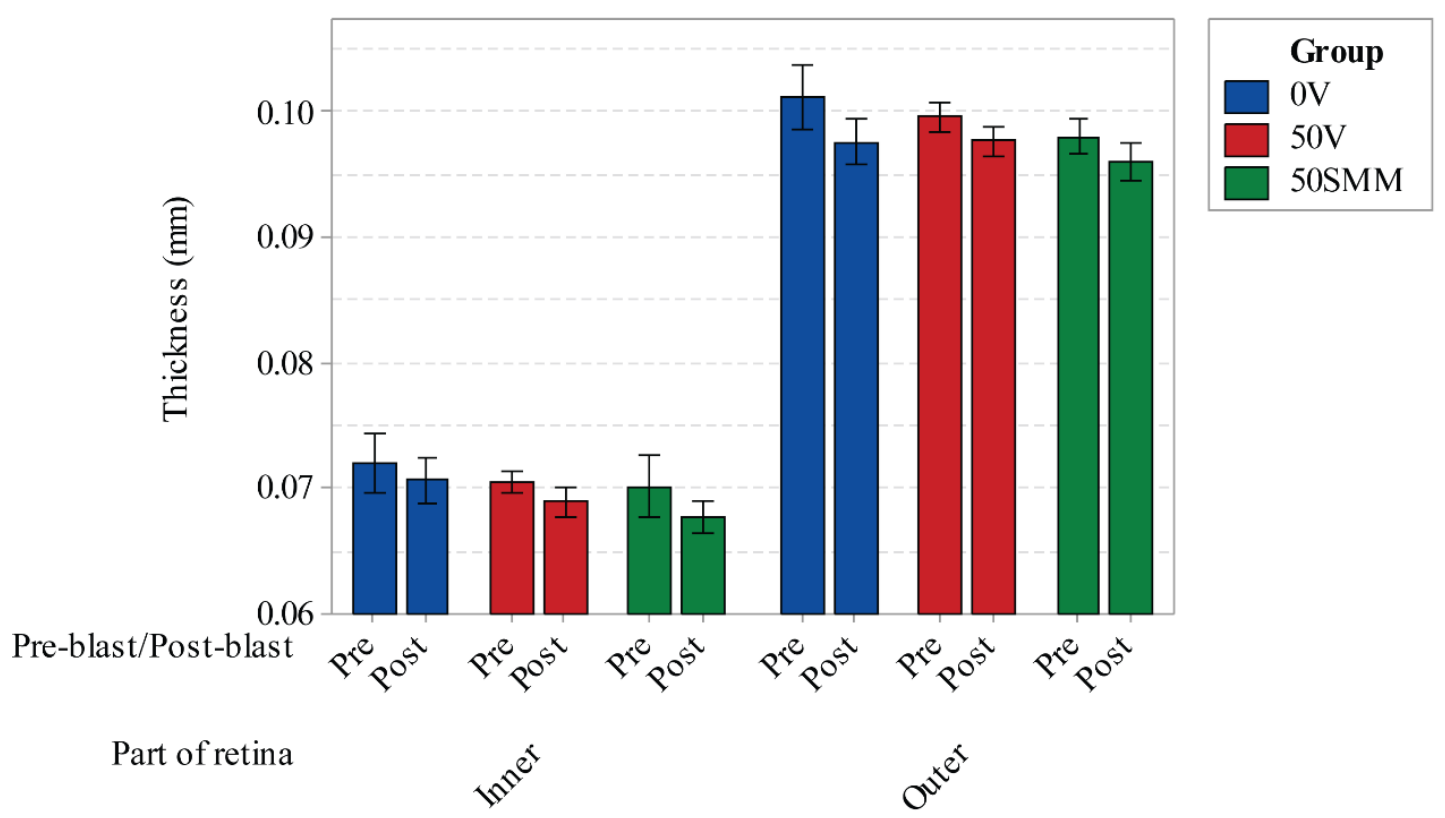

Figure 5-2. Right eye pre-blast and post-blast OCT measurements.

The right eye OCT measurements were not significantly altered in any group after blast. Bars are one standard error from the mean. 


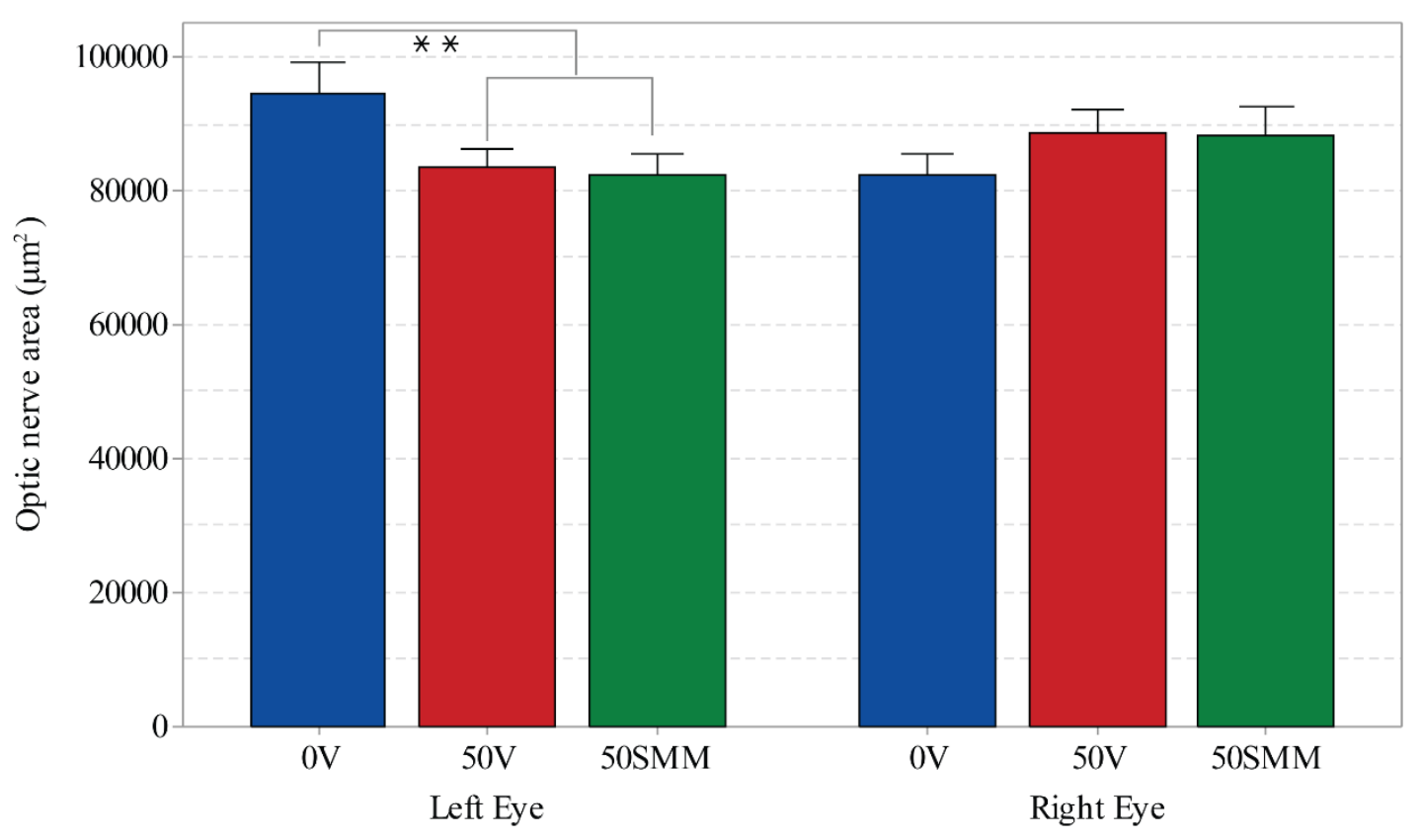

Figure 5-3. Optic nerve cross-sectional area at 11 weeks after blast.

Cross-sections of optic nerves were measured circumferentially for area. In the left eye, $50 \mathrm{~V}$ animals showed a significant atrophy that was not remedied by SMM-189. The right eye showed no such atrophy, and all groups were statistically equivalent in right eye cross-sectional area. $* *$ denotes significant difference between $0 \mathrm{~V}$ LE cross sectional area and both $50 \mathrm{~V}(\mathrm{p}=0.0393)$, and 50SMM $(\mathrm{p}=0.028)$. Bars are one standard error from the mean. 


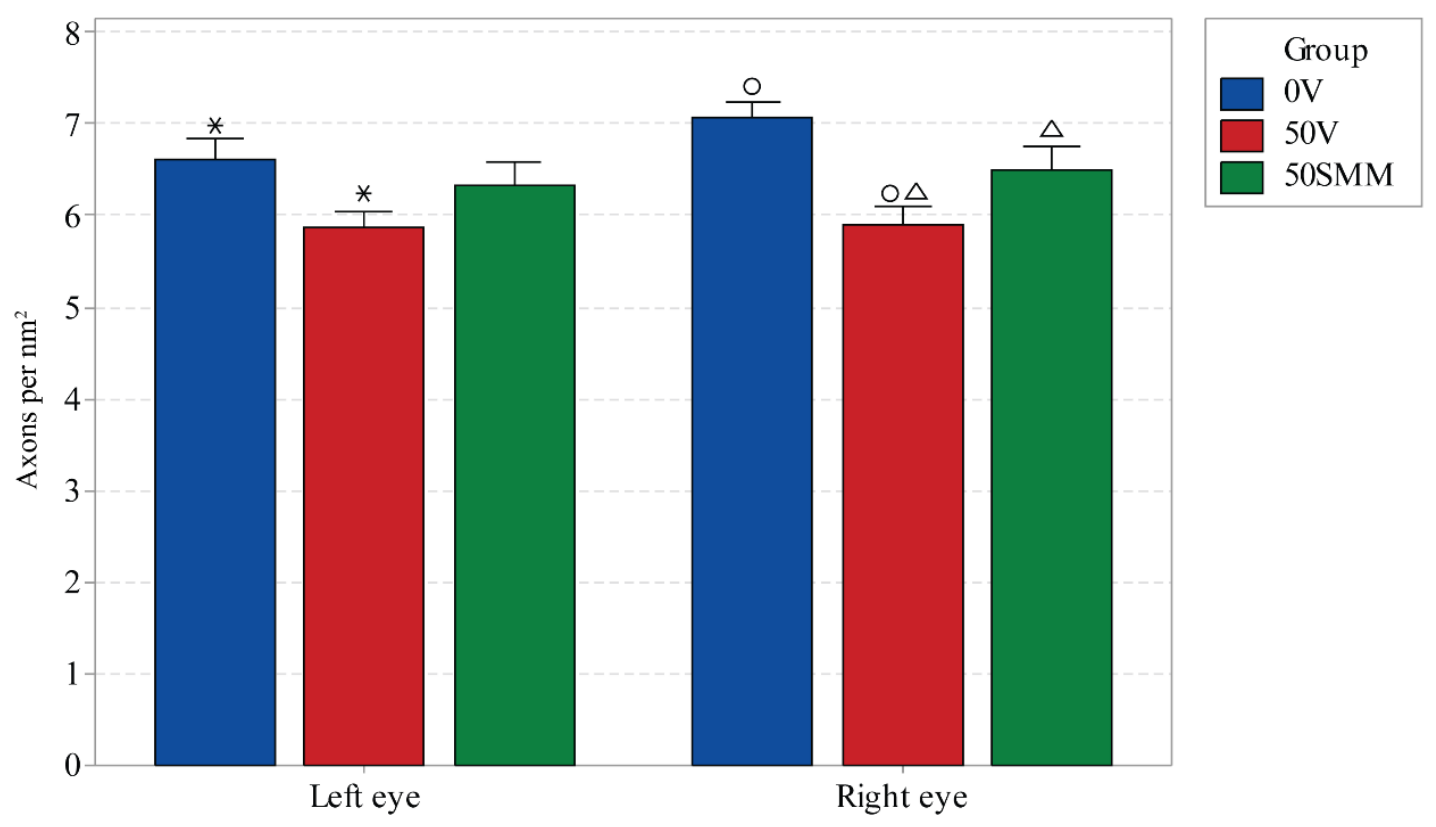

Figure 5-4. Axon density in the optic nerve at 11 weeks after blast.

The axon density was determined from manual counts of axons from four regions of the optic nerve in cross-sections. The counts from these four quadrants were averaged for the axon density measurements shown in this figure. Both the left and right eyes of $50 \mathrm{~V}$ animals had a significant decrease in axon density, which the graph suggests was remedied with SMM-189 treatment. The SMM-189 rescue was not significant in the left eye, but showed a clear trend, and was significant in the right eye. Asterisk denotes significant difference between $0 \mathrm{~V}$ and $50 \mathrm{~V}$ LE axon density $(\mathrm{p}=0.0198)$. Circle denotes significant difference between $0 \mathrm{~V}$ and $50 \mathrm{~V} R E$ axon density $(\mathrm{p}=0.0002)$. Triangle denotes significant difference between 50V and 50SMM RE axon density $(\mathrm{p}=0.0438)$. Bars are one standard error from the mean. 


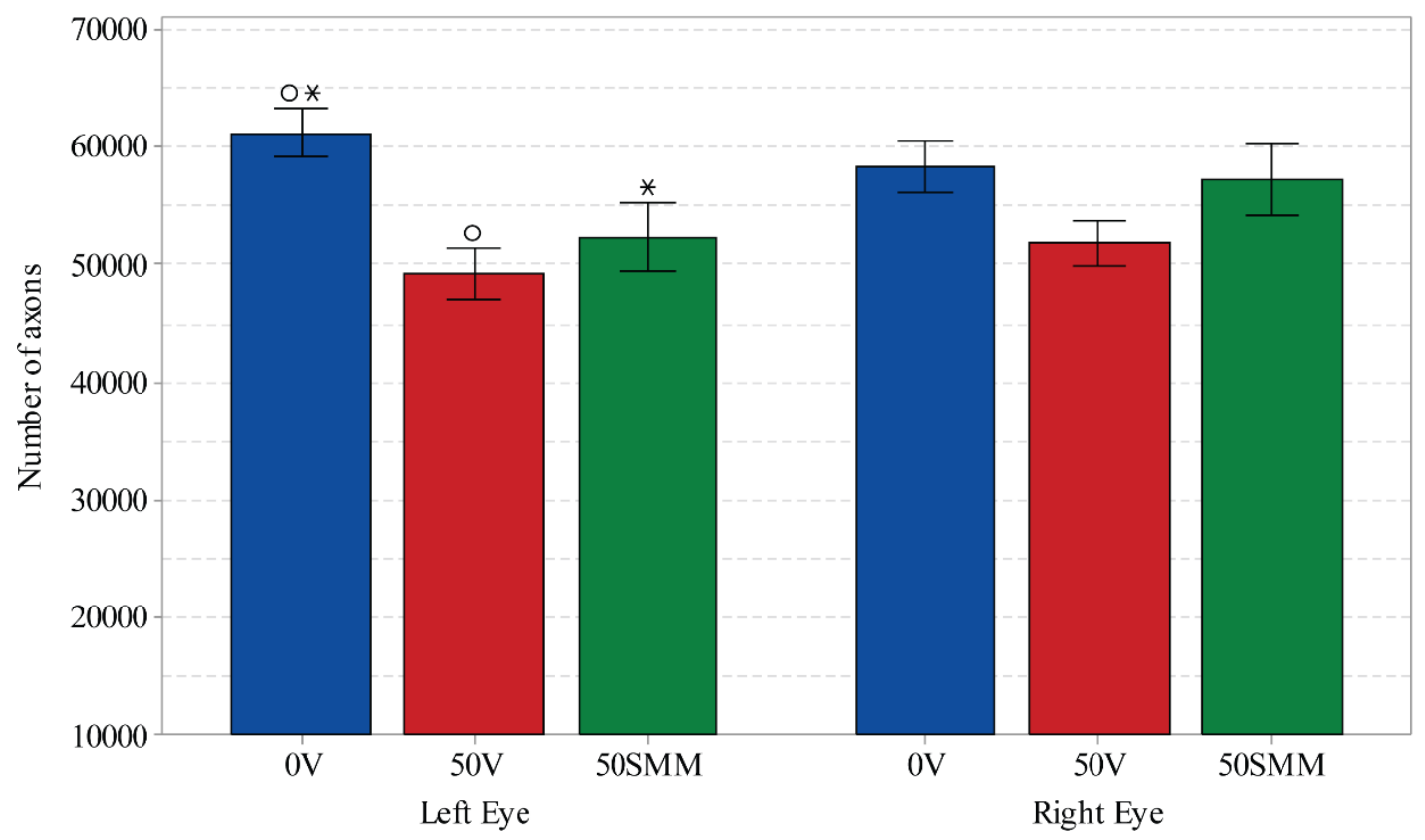

Figure 5-5. Total axons in the optic nerve 11 weeks after blast.

This figure shows the extrapolated axon counts in the optic nerve for each group. The total number of axons was calculated from the area and the axon density for each animal, and these numbers were averaged for all groups. The number of axons in the left $\mathrm{ON}$ of $50 \mathrm{~V}$ animals was significantly lower than controls, and this was partially rescued by drug treatment. In the right eye, the $50 \mathrm{~V}$ animals showed a decrease in axon number that was nearly significant compared to controls. The drug treated animals were undistinguishable from controls. Circle denotes significant difference between $0 \mathrm{~V}$ and 50V LE total axon number $(\mathrm{p}=0.0014)$. Asterisk denotes significant difference between 0V and 50SMM LE total axon number $(\mathrm{p}=0.0136)$. Bars are one standard error from the mean. 


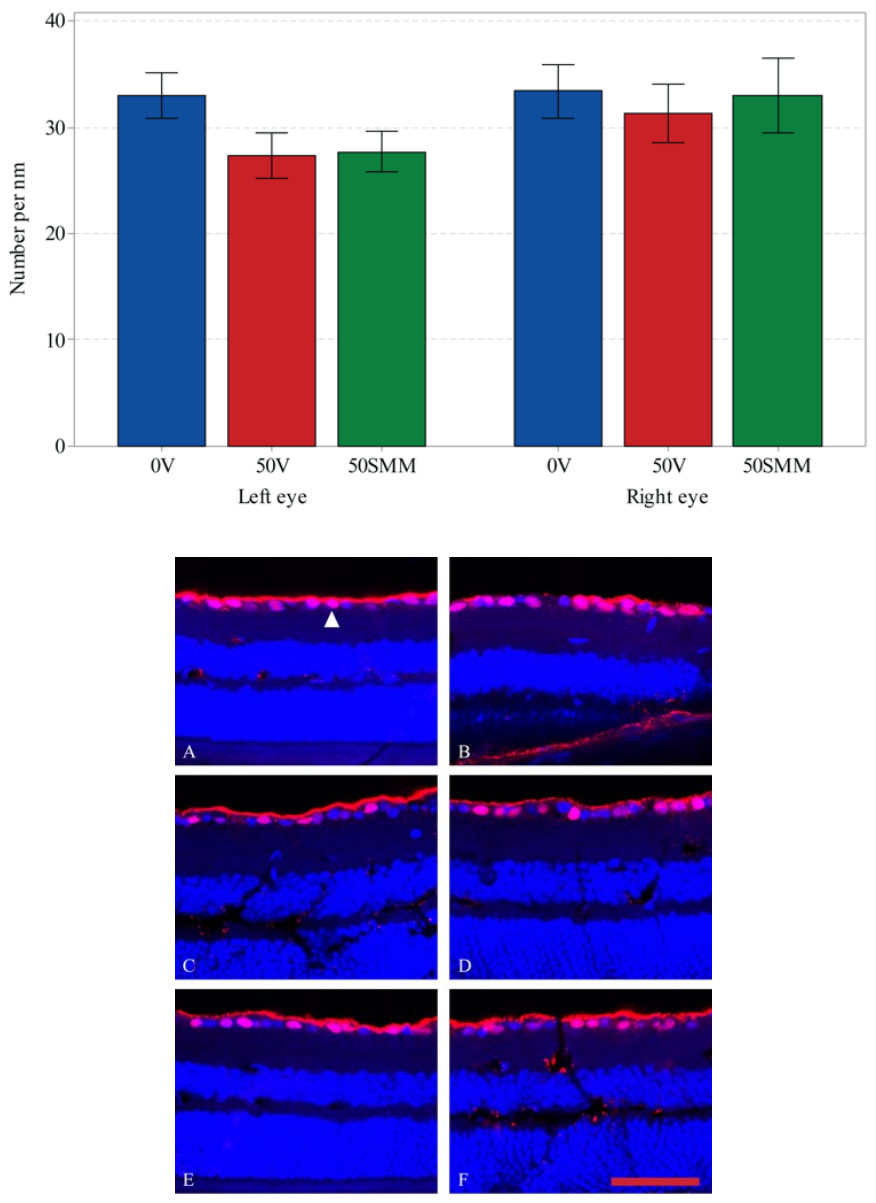

Figure 5-6. Brn3a+ retinal ganglion cell counts in the retina at 30 days after blast.

Brn3a immunohistochemistry was used to quantify RGCs in the retina (A-F). Representative sections of double-labeled retinas showing Brn3a+ cells (red) and DAPI+ nuclei (blue) are provided for $0 \mathrm{~V}$ left and right eyes (A, B), 50V left and right eyes (C, $\mathrm{D})$, and 50SMM left and right eyes (E, F). The scalebar is $50 \mu \mathrm{m}$. The graph shows $\mathrm{Brn} 3 \mathrm{a}+$ cell quantification from all sections, with the left eye of $50 \mathrm{~V}$ animals having a near significant decrease in the number of RGCs compared to 0V animals. Drug treated animals were indistinguishable from $50 \mathrm{~V}$ animals in the left eye. The right eye showed no changes across all groups, although the $50 \mathrm{~V}$ group showed a trend toward fewer RGCs than the $0 \mathrm{~V}$ and 50SMM animals. Bars are one standard error from the mean. 


\section{Iba-1 counts}

3 days after blast. The left eye of $50 \mathrm{~V}$ animals showed a significant increase in number of Iba-1+ cells compared to both $0 \mathrm{~V}$ and 50SMM animals, by one-way ANOVA $(p=0.0004$ and $p=0.0014$, respectively). The 50SMM animals had a decrease in number toward controls, but were still significantly different than control $(\mathrm{p}=0.0079)$. The right eyes were statistically equivalent for all groups by one-way ANOVA ( $p=0.9995)$, although it is important to note that the 50SMM group had only an $n=1$ (Figure 5-7).

30 days after blast. At 30 days out from blast, in the left eye, the number of Iba$1+$ cells was significantly greater in 50V animals than in both $0 \mathrm{~V}$ and 50SMM animals by one-way ANOVA ( $p=0.0001$, and $p<0.0001$, respectively), although not as numerous as at the 3 day time point. No significant difference was found between the $0 \mathrm{~V}$ and the 50SMM groups for the left eye. The right eye showed a similar result, with the number of IBa- $1+$ cells in $50 \mathrm{~V}$ being significantly greater than both $0 \mathrm{~V}$ and $50 \mathrm{SMM}(\mathrm{p}<0.0001$ and $p=0.0112$, respectively). The 50SMM and $0 \mathrm{~V}$ groups were nearly significantly different at $\mathrm{p}=0.0779$ (Figure 5-8).

Qualitative observations. In $50 \mathrm{~V}$ animals 3 days after blast, microglia in the left eye appeared to penetrate further into the outer layers of the retina when compared to control eyes, and their processes seemed more numerous throughout the whole of the retina. Drug-treated left eyes had fewer microglia and fewer processes visible, but they still penetrated into the outer layers of the retina like the $50 \mathrm{~V}$ animals. Both $50 \mathrm{~V}$ and 50SMM animals had a large number of vertically oriented microglia, which appeared to reach across retinal layers. These vertically oriented microglia were not evident in $0 \mathrm{~V}$ animals.

In control retinas 30 days after blast, both eyes showed distinctive stellate microglia present in the IPL. A majority of the cells visible in control retinas were horizontally oriented and for the most part were confined to the inner layers of the retina in both eyes. Conversely, $50 \mathrm{~V}$ animals showed many vertically oriented cells in both eyes, with some penetrating into the outer retinal layers. The more horizontally oriented cells in the left eyes of 50V animals had a characteristic lightning bolt appearance, and were unlike those seen in control retinas. Drug treated animals also had many vertically oriented cells in both eyes, with fewer cells penetrating into the outer retinal layers than the $50 \mathrm{~V}$ animals. The horizontally oriented cells in the left eyes of 50SMM animals generally had a lightning bolt appearance similar to those of the $50 \mathrm{~V}$ animals. However, the right eyes of 50SMM animals contained cells with thicker processes that did not have the characteristic lightening bolt appearance, and instead had thicker processes that tended to branch in all directions.

\section{GFAP quantification}

7 days after blast. Neither the left nor the right eyes were significantly different for any group at 7 days by one-way ANOVA analysis (Figure 5-9). 


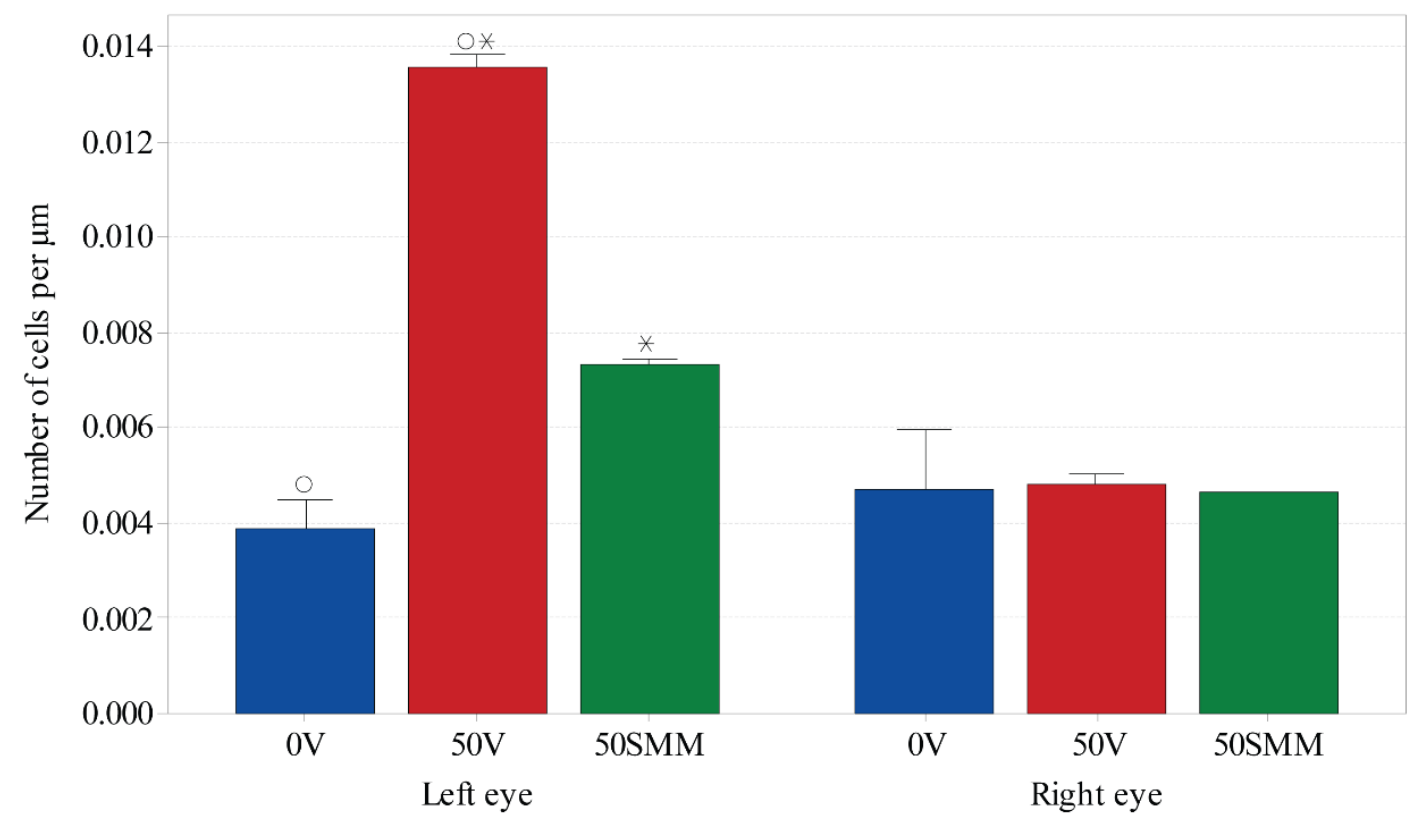

Figure 5-7. Microglial cell counts in the retina at 3 days after blast.

Iba-1 immunostaining was used to visualize and quantify microglia in the retina at 3 days after blast. The left eye of $50 \mathrm{~V}$ animals showed a large increase over control animals, which was highly significant. SMM-189 treatment remedied this significantly, with a return toward control numbers. The right eyes of all groups were indistinguishable from one another at this time point. Circle denotes significant difference between $0 \mathrm{~V}$ and $50 \mathrm{~V}$ LE microglial counts $(\mathrm{p}=0.0004)$. Asterisk denotes significant difference between $50 \mathrm{~V}$ and 50SMM LE microglial counts $(\mathrm{p}=0.0014)$. Bars are one standard error from the mean. 


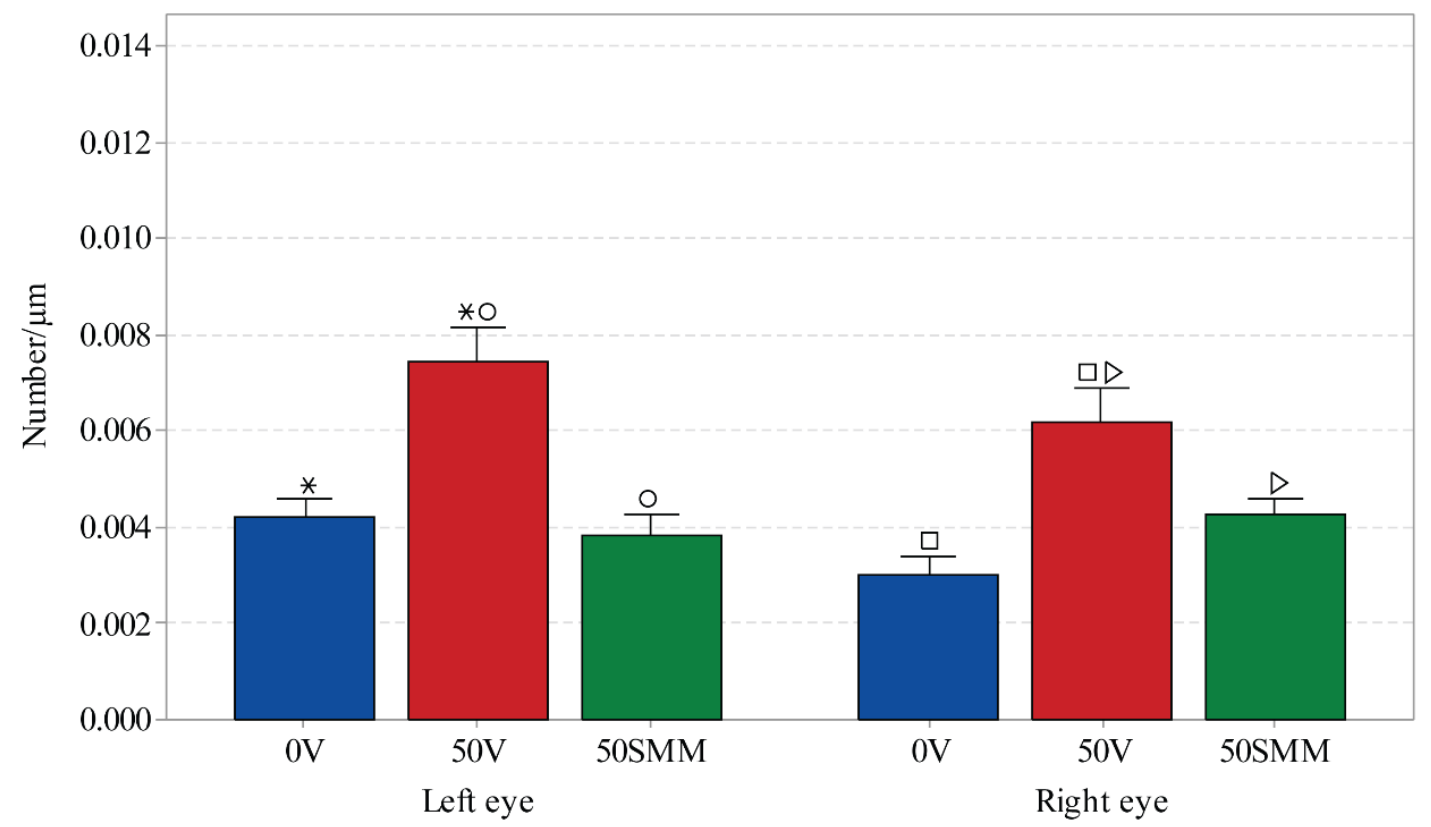

Figure 5-8. Microglial cell counts in the retina at 30 days after blast.

At 30 days after blast, microglia in the left eye of blasted animals were decreased from their 3 day numbers, but were still significantly elevated when compared to controls. Again, the SMM-189 treatment rescued this, with the 50SMM and 0V animals being indistinguishable from one another. Surprisingly, the right eye of $50 \mathrm{~V}$ animals at this time point had an elevation of microglia that were significantly greater than in controls and drug-treated animals. The number of microglia in the two eyes of $50 \mathrm{~V}$ animals were similar, with slightly more in the left eye. Asterisk denotes significant difference between $0 \mathrm{~V}$ and $50 \mathrm{~V}$ LE microglial counts $(\mathrm{p}=0.0001)$. Circle denotes significant difference between 50V and 50SMM LE microglial counts $(p<0.0001)$. Square denotes significant difference between $0 \mathrm{~V}$ and $50 \mathrm{~V}$ RE microglial counts $(\mathrm{p}<0.0001)$. Triangle denotes significant difference between 50V and 50SMM RE microglial counts $(p=0.0112)$. Bars are one standard error from the mean. 


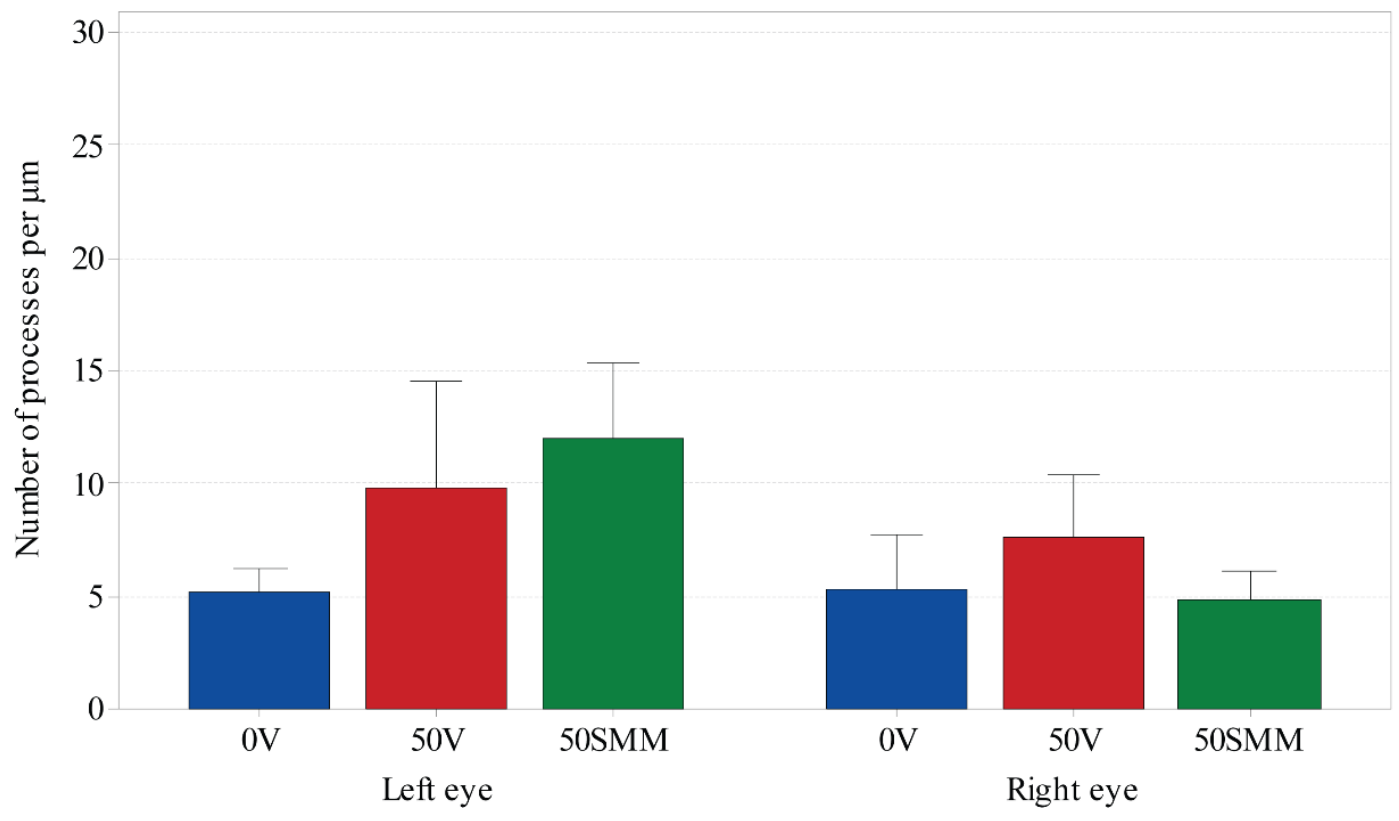

Figure 5-9. Müller cell glial reactivity in the retina at 7 days after blast.

Müller cell glial reactivity was visualized and quantified using GFAP immunostaining. Processes penetrating the inner retina past the IPL were counted and averaged to yield the numbers in this figure. At this time point, there was a trend in both left and right eyes of $50 \mathrm{~V}$ animals showing more GFAP reactivity than controls, but this was not significant. Interestingly, the left eye of drug treated animals was slightly increased over $50 \mathrm{~V}$ animals, but again, not significantly so. Bars are one standard error from the mean. 
30 days after blast. Left eyes of $50 \mathrm{~V}$ animals had significantly more GFAP processes that extended sclerad from the RGC layer than either $0 \mathrm{~V}$ or $50 \mathrm{SMM}$ animals by one-way ANOVA ( $\mathrm{p}=0.0101$ and $\mathrm{p}=0.024$, respectively), while $0 \mathrm{~V}$ and $50 \mathrm{SMM}$ were not significantly different from one another. The right eye showed no significant differences between any group (Figure 5-10). A Pearson correlation revealed a slight negative correlation $(\mathrm{r}=-0.196)$ between the number of reactive GFAP processes in the retina at 30 days and the number of Brn3a immunopositive cells in the same eye at this time, but it was not significant $(\mathrm{p}=0.5613)$.

Qualitative observations. The control animals showed a thin region of GFAP staining in the NFL of the retina in both eyes, and occasional NFL regions with little or no staining at all. In the left eye of $50 \mathrm{~V}$ animals, there were numerous GFAP positive processes that penetrated into the IPL and sometimes into the outer retina. The NFL in the $50 \mathrm{~V}$ left eyes showed variable regions of thicker GFAP reactivity. The right eye of $50 \mathrm{~V}$ animals had many fewer GFAP processes that penetrated into the IPL than the left eye, but still had a variable presence of thickened GFAP staining in the NFL. The 50SMM animals looked similar to control animals in both eyes, with little or no penetration of Müller cell processes past the NFL, and no evident thickening of the GFAP staining in the NFL.

\section{M1/M2 markers in the right OT}

The 50V and 50SMM groups were both significantly different from $0 \mathrm{~V}$ in the number of M1 and M2-biased cells using Chi square goodness-of-fit testing ( $<<0.0001$ for both). They were also significantly different from one another $(\mathrm{p}<0.0001)$. The abundance of M1-biased cells was highest in the $50 \mathrm{~V}$ animals, at nearly $90 \%$ of the cells quantified. In 50SMM animals, this number dropped to just below $70 \%$. Accordingly, the M2-biased cell abundance in $50 \mathrm{~V}$ animals was very low, with only $6.5 \%$ of cells being M2-biased, contrasted with 50SMM animals who had about $25.5 \%$ of cells being M2-biased. In control $0 \mathrm{~V}$ animals, half of the cells quantified were M1-biased, with almost $28 \%$ being M2-biased. The frequencies of M1 and M2-biased microglial cells in the different experimental groups are shown in Figure 5-11.

\section{Axon bulbs}

Axon bulbs were present in significantly higher numbers at 3 days after blast in $50 \mathrm{~V}$ animals when compared to controls (one-way ANOVA, $\mathrm{p}=0.0059$ ), and were lessened in 50SMM animals (Figure 5-12). This decrease was nearly significant by oneway ANOVA $(p=0.0540)$. The 50 SMM and $0 \mathrm{~V}$ animals were not statistically different from one another at 3 days. No SMM treated animals were available at 5 or 7 days, so only $0 \mathrm{~V}$ and $50 \mathrm{~V}$ animals were compared at these time points. A two-way ANOVA showed a significant difference between the $0 \mathrm{~V}$ and $50 \mathrm{~V}$ animals across all 3 time points after blast $(\mathrm{p}=0.0341)$ (Figure 5-13). A Pearson correlation revealed a strong negative correlation ( $\mathrm{r}=-0.8414)$ between the number of axon bulbs in the ROT of 50SMM 


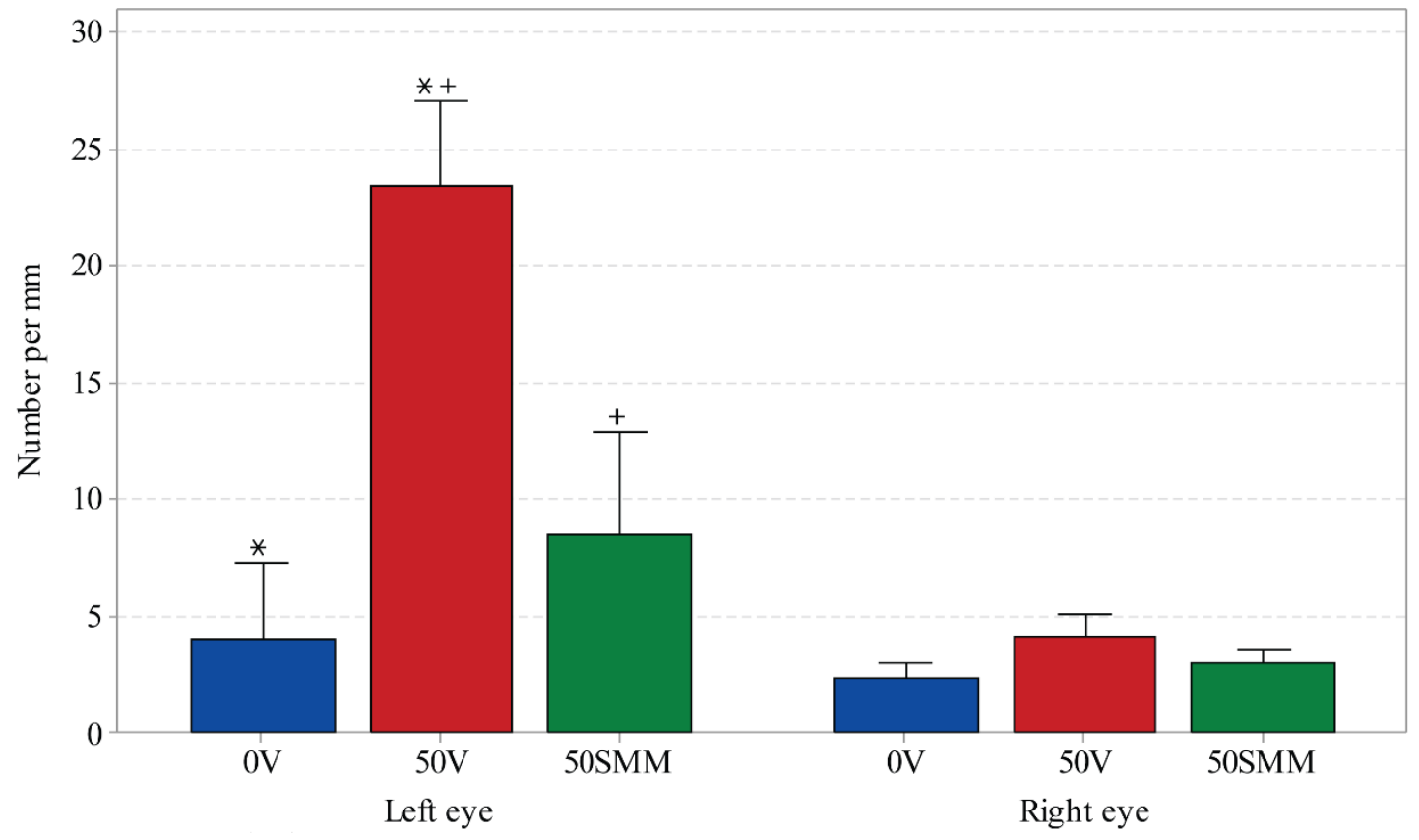

Figure 5-10. Müller cell glial reactivity in the retina at 30 days after blast.

At 30 days after blast, the left eye of $50 \mathrm{~V}$ animals showed a clear and significant increase in the number of reactive Müller cell processes, which was also significantly remedied by drug treatment. The right eye levels were nearly identical for all three groups. Asterisk denotes significant difference between $0 \mathrm{~V}$ and 50V LE GFAP+ processes $(\mathrm{p}=0.0101)$. Plus sign denotes significant difference between 50V and 50SMM LE GFAP+ processes $(\mathrm{p}=0.024)$. Bars are one standard error from the mean. 


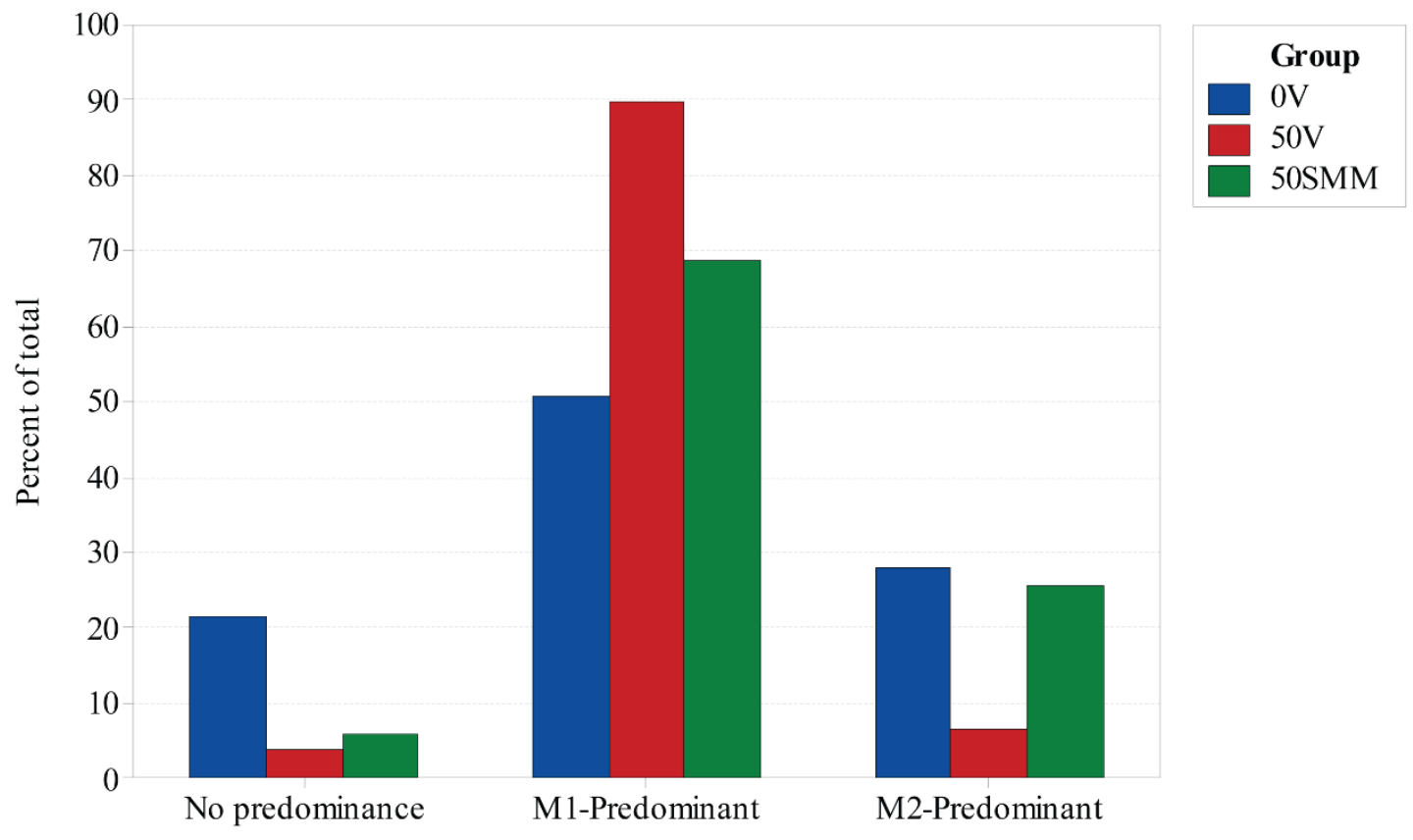

Figure 5-11. Microglial polarization in the right optic tract at 3 days after blast.

At 3 days after blast, immunostaining for CD16/32 (M1 marker) and CD206 (M2 marker) was used to categorize microglial polarization in the right optic tract. Individual cells were categorized based on their predominant marker, with cells expressing equal amounts of both placed into the no predominance category. The control animals had the highest number of cells with no predominant surface marker, where the 50V and 50SMM animals had very few. The $50 \mathrm{~V}$ animals had a large increase in M1 pro-inflammatory microglia over the controls, with much fewer M2 anti-inflammatory than $0 \mathrm{~V}$ animals. Drug treatment decreased the number of M1-predominant microglia, but increased the number of M2-predominant, suggesting the drug may indeed act to alter polarization of microglia into a more anti-inflammatory phenotype. The slight increase in the number of non-predominant cells in SMM-189 treated animals may signify a group of cells who are shifting polarization from M1 to M2. 


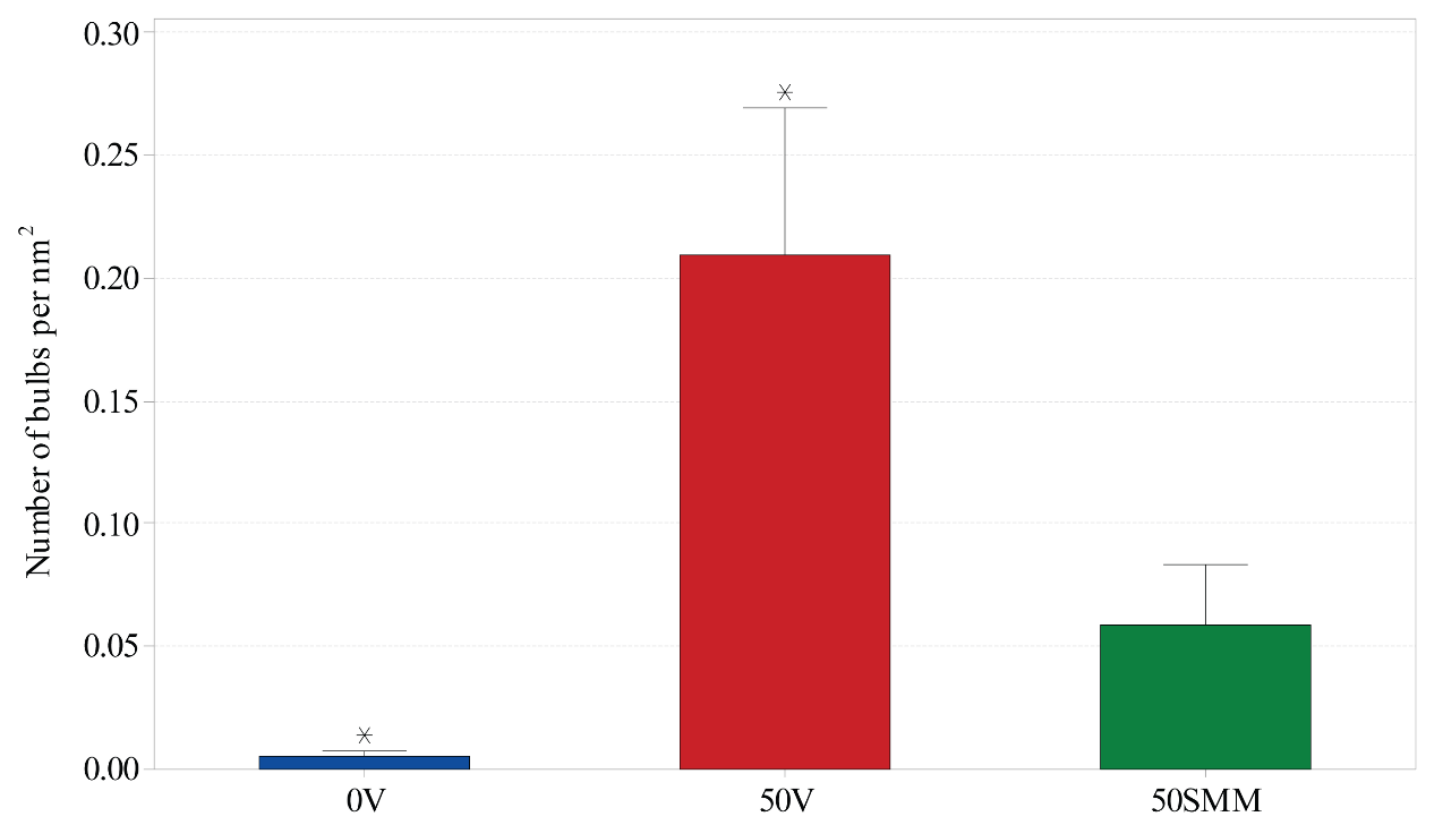

Figure 5-12. Axon bulbs in the right optic tract at 3 days after blast.

EYFP reporter mice were used to quantify axon bulbs at 3 days after blast in the right optic tract. The right optic tract contains fibers that originate mostly from the left optic nerve and retina. In $50 \mathrm{~V}$ animals, there was a very large number of pathologic axon bulbs present, where $0 \mathrm{~V}$ animals had almost none. Drug treatment decreased the number of bulbs drastically, although they were not significantly different than $50 \mathrm{~V}$ animals. The 50SMM animals were statistically equivalent to $0 \mathrm{~V}$ animals. Asterisk denotes significant difference between $0 \mathrm{~V}$ and $50 \mathrm{~V}$ axon bulbs in the ROT $(\mathrm{p}=0.0059)$. Bars are one standard error from the mean. 


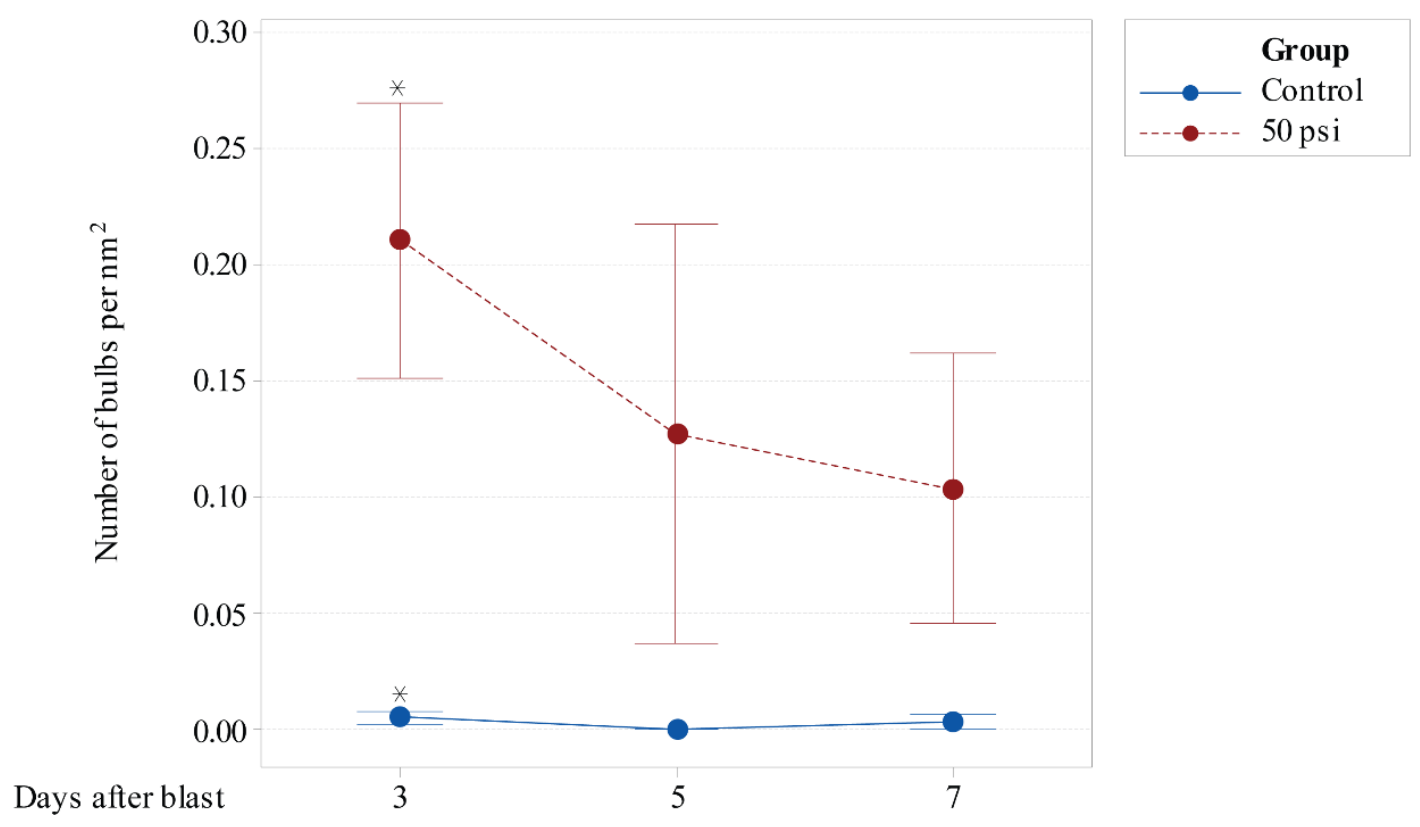

Figure 5-13. The number of axon bulbs in the right optic tract over the first week after blast.

The number of axon bulbs was quantified in EYFP reporter mice and using SMI-32 IHC at 3,5, and 7 days after blast in 50-psi and control animals. These counts showed the number of bulbs in the ROT of 50-psi animals is highest at 3 days after blast, then decreases at 5 days, and 7 days. The sham blasted animals had nearly zero bulbs at all three time points. The number of bulbs over these three days was significantly different from the sham-blasted animals. Asterisk denotes significant difference between $0 \mathrm{~V}$ and $50 \mathrm{~V}$ axon bulbs in the ROT at all three time points after blast $(\mathrm{p}=0.0341)$. Bars are one standard error from the mean. Data not available for 50SMM animals after 3 days postblast. 
animals at 3 days and the number of M2-biased microglia in the ROT at this time, but it was not significant $(\mathrm{p}=0.3635)$.

\section{Summary}

The retinas of blasted animals show signs of both acute, and sustained inflammation. At 3 days post-blast, the number of microglia is increased significantly when compared to controls. This is remedied by drug treatment, with a decrease in the number of microglia in the 50SMM animals when compared to $50 \mathrm{~V}$ animals. At 7 days, retinal Müller glia show no obvious signs of reactivity, however at 30 days, left eyes of $50 \mathrm{~V}$ animals show a significant GFAP response over controls. This response is quelled by drug treatment, such that there was no statistical difference between 50SMM and $0 \mathrm{~V}$ animals. In addition, both eyes of $50 \mathrm{~V}$ animals show an increased number of microglia, with a drug rescue returning the numbers toward that of controls. There are some hints that RGCs may be lost due to the injury, with Brn3a+ cell counts in the RGC layer showing a near-significant decrease in the left eye of 50V animals; however this trend does not appear to be remedied by drug treatment. Pre-blast and post-blast OCT measurements show significant thinning in the LE outer retina of blasted animals. Drugtreated animals show no such change, but have a significant decrease in the inner retina of the left eye. Control animals have statistically unchanged pre-blast and post-blast measurements for both eyes.

In the optic tract of 50V animals at 3 days, axon bulbs are visible in large quantities, and to a lesser extent are still present in 50SMM animals. Quantification in $50 \mathrm{~V}$ animals shows that at 5 and 7 days, the number of bulbs in the ROT dissipate, and the character of the bulbs appears more granular. The number of axon bulbs in the ROT is consistently higher in $50 \mathrm{~V}$ animals than $0 \mathrm{~V}$ animals at all 3 time points examined during the first week after blast. Also in the ROT, at 3 days post-blast, the number of microglia displaying predominantly inflammatory (M1) markers is significantly higher in $50 \mathrm{~V}$ animals than both controls and drug treated animals. Conversely, SMM-treated animals show an increase in the number of cells displaying a bias toward antiinflammatory (M2) markers, with an apparent shift of about $20 \%$ of cells from M1 to M2-biased markers.

Finally, at 2 months after blast, optic nerves of the left eye were atrophic in $50 \mathrm{~V}$ and 50SMM animals, with a concurrent loss in total axon number for both groups in this eye. Interestingly, the LE axon density in the 50SMM animals was unchanged from controls, while the $50 \mathrm{~V}$ animals had a significant decrease in axon density. The right $\mathrm{ON}$ of $50 \mathrm{~V}$ animals showed a significant decrease in axon density, but the decrease in total axon number was not significantly different from controls. See Table 5-2 for a comprehensive summary of all structural and functional results. 
Table 5-2. Summary of findings.

\begin{tabular}{llll}
\hline Endpoint examined & Without drug & With drug & Comments \\
& $50-$ psi + vehicle & $50-$ psi + SMM-189 & \\
& versus & versus & \\
& sham + vehicle & sham + vehicle & \\
\hline
\end{tabular}

\section{Functional endpoints}

\begin{tabular}{|c|c|c|c|}
\hline ERG A-wave & LE increased & - & No change at one month \\
\hline ERG B-wave & $\begin{array}{l}\text { LE increased* RE } \\
\text { increased }\end{array}$ & - & $\begin{array}{l}\text { B-wave amplitude increased in } 50 \mathrm{~V} \text { LE, RE } \\
\text { nearly significant }\end{array}$ \\
\hline $\begin{array}{l}\text { Optical coherence } \\
\text { tomography }\end{array}$ & $\begin{array}{l}\text { LE outer retina } \\
\text { decreased* }\end{array}$ & $\begin{array}{l}\text { LE inner retina } \\
\text { decreased* }\end{array}$ & $\begin{array}{l}\text { Drug treatment improved LE photoreceptor } \\
\text { damage }\end{array}$ \\
\hline Visual acuity threshold & - & - & No change at one month \\
\hline $\begin{array}{l}\text { Contrast sensitivity } \\
\text { threshold }\end{array}$ & Decreased* & - & $\begin{array}{l}\text { RE more severely affected than LE one } \\
\text { month after blast }\end{array}$ \\
\hline blogy & & & \\
\hline $\begin{array}{l}\text { Optic nerve axon } \\
\text { density }\end{array}$ & Decreased* & Decreased & $\begin{array}{l}\text { Near significant difference in RE of 50SMM } \\
\text { compared to } 0 \mathrm{~V}\end{array}$ \\
\hline $\begin{array}{l}\text { Brn- } 3 a+\text { cell counts in } \\
\text { the retina }\end{array}$ & Decreased & Decreased & $\begin{array}{l}\text { Near significant difference in LE of both } 50 \mathrm{~V} \\
\text { and 50SMM }\end{array}$ \\
\hline
\end{tabular}


Table 5-2. Continued.

\begin{tabular}{|c|c|c|c|}
\hline Endpoint examined & $\begin{array}{l}\text { Without drug } \\
50 \text {-psi + vehicle } \\
\text { versus } \\
\text { sham + vehicle }\end{array}$ & $\begin{array}{l}\text { With drug } \\
\text { 50-psi + SMM-189 } \\
\text { versus } \\
\text { sham + vehicle }\end{array}$ & Comments \\
\hline $\begin{array}{l}\text { GFAP reaction in the } \\
\text { retina }\end{array}$ & Increased* & - & $\begin{array}{l}\text { Significant difference between } 50 \mathrm{~V} \text { and } \\
50 \mathrm{SMM} \text { in LE }\end{array}$ \\
\hline $\begin{array}{l}\text { Iba- } 1+\text { cell counts in } \\
\text { the retina }\end{array}$ & Increased* & - & $\begin{array}{l}\text { Significant increase in } 50 \mathrm{~V} \text { LE at } 3 \text { and } 30 \\
\text { days, RE increased at } 30 \text { days }\end{array}$ \\
\hline $\begin{array}{l}\text { M1/M2 counts in right } \\
\text { OT }\end{array}$ & M1 increased* & $\begin{array}{l}\text { M1 increased* } \\
\text { M2 equal }\end{array}$ & $\begin{array}{l}\text { SMM-189 significantly increased the number } \\
\text { of M } 2 \text { microglia compared to } 50 \mathrm{~V}\end{array}$ \\
\hline $\begin{array}{l}\text { EYFP bulbs in the right } \\
\text { OT }\end{array}$ & Increased* & - & $\begin{array}{l}\text { 50SMM and } 0 \mathrm{~V} \text { not significantly } \\
\text { different from one another }\end{array}$ \\
\hline
\end{tabular}

*Denotes statistical significance $\leq p=0.05$.

- means no change.

Italics indicate an evident trend. 


\section{CHAPTER 6. DISCUSSION}

In Chapter 2, we proposed a set of experiments to investigate visual dysfunction in our mouse model of overpressure blast-induced TBI, and to test the hypothesis that a major pathway of the disease is immune-mediated secondary injury. While previous studies had demonstrated significant immune involvement after injury, specifically in areas important to the visual system in the brain, little information existed about the pathology within the eye itself. The histopathologic studies in this dissertation have revealed much about the underlying damage to the eye in this model, and these will now be discussed in more detail.

\section{The Elucidated Histopathology of mTBI-Associated Visual Dysfunction}

\section{The retina}

In our previous studies, the retina of blasted animals at 10 weeks after blast showed signs of significant damage, in the form of thinning in the left eye, and thickening in the right eye ${ }^{179}$. The left eye thinning was due in part to thinning within the INL, but this could not account for the total retinal thickness loss. Little was previously known about what happened in the retina at more acute time points, and thus it became a pertinent focus of this dissertation work to examine the retina at earlier time points after blast.

Microglial inflammatory response. Significantly, it was found that within 3 days of blast exposure, the left retinas of blasted animals already showed signs of a substantial microglial response. Quantification in the left retina demonstrated that blast animals had an increase in microglial density that was nearly triple that of controls. Counts from the nasal and temporal side of the same eye at the level of the optic nerve head were statistically equivalent, indicating a seemingly uniform increase in microglial presence across the middle retina. The morphology of the microglia in left eyes after blast was more robust, with darker staining and thicker, less complex processes.

Retinal microglia have a somewhat different morphology than that of brainresident microglia. At physiological conditions, retinal microglia are found predominantly in the inner retinal layers ${ }^{221}$ and have thinner, longer, and more complex processes ${ }^{222,223}$. Under pathological conditions, even very early in disease pathogenesis, subtle morphologic changes can occur in retinal microglia that indicate a reaction to inflammatory stimuli ${ }^{223,224}$. Processes shorten, as in brain microglia, but rarely to the point of being fully retracted with an amoeboid appearance. Microglia will begin to migrate from their normal location in the inner retina to the outer retinal layers ${ }^{221}$, using the Müller cell glia as scaffolding, and interacting with them to regulate the immune response ${ }^{225}$. This gives them a more vertical appearance (i.e. vitread to sclerad), making them thus relatively easy to identify compared to the normal horizontally-oriented and 
stellate microglial cells in physiological conditions. Microglia in the left eyes of $50 \mathrm{~V}$ animals 3 days after blast appeared to penetrate further into the outer layers of the retina when compared to control left eyes, and their processes seemed more numerous throughout the whole of the retina. Additionally, there were numerous vertically-oriented microglial processes in 50V left eyes, whereas controls had none (Figure 6-1A and B). These observations taken together suggest that microglia were reacting to an inflammatory micro-environment by polarizing and traversing the retinal layers in response to cytokine and chemokine signals ${ }^{226}$. Interestingly, the drug treated animals only showed a marginal, non-significant increase in microglial counts over that of controls, but their microglia still appeared to have more vertically-oriented processes (Figure 6-1C). As discussed in Chapter 2, research on CB2 inverse agonists has shown them to decrease leukocyte recruitment ${ }^{227}$, which may be the reason for the decrease in microglia numbers in drug-treated animals. However, it cannot be ruled out that cessation of microglial proliferation is instead the cause of this drug benefit, since the mechanism of microglial increase after blast injury is not currently known. Because SMM-189 is thought to selectively polarize microglia to the M2 state, their altered morphology indicates that perhaps they are still interacting with the Müller cell glia but in this case to organize a more neuroprotective immune response. At the same time that the left eye showed signs of significant microglial response in $50 \mathrm{~V}$ animals, the right eyes of blasted animals showed no such reactive increase, with statistically equivalent numbers of microglia as controls. The morphology of microglia in the right eye also appeared more consistent with those of controls, with fewer processes visible, and no apparent vertically-oriented processes.

At 30 days out from blast, the LE microglial presence was still evident, albeit diminished by a little over $50 \%$ from day 3 levels. Microglial processes at this point were still clearly more abundant throughout the inner retinal layers than in $0 \mathrm{~V}$ animals, and similarly to the 3-day retinas, had many vertically-oriented processes (Figure 6-2C). In the right eye, while there were no signs of inflammation early on, at this 30-day time point there was a significant increase in microglial density that was only marginally lower than that of the left eye. Interestingly, although the drug treated animals had a significant decrease in the number of microglia present in the retinas when compared with $50 \mathrm{~V}$ animals, the morphologic characteristics of the microglia appeared more similar to the $50 \mathrm{~V}$ animals than they did to controls (Figure 6-2A, $\mathbf{C}$, and $\mathbf{E}$ ). Their processes appeared thicker overall, as in $50 \mathrm{~V}$ animals, but they had many more vertically-oriented cells present, even more so than in 3 day $50 \mathrm{~V}$ animals. Aside from vertically-oriented cells, the microglia in the left eyes of 50SMM animals looked similar to that of $50 \mathrm{~V}$ animals, with a lightning bolt appearance, and medium-density processes that were sometimes relatively long. However, in the right eye of 50SMM animals, cells appeared thicker and more robust, much different than in any of the other retinas at this time point (Figure 6-2B, D, and F). Control $0 \mathrm{~V}$ animals had highly dendritic, star-shaped microglia present predominantly in the IPL, with thinner, more ornate processes (Figure 6-2A and B). These types of cells were not seen in either eye of 50V or 50SMM animals.

The persistence of microglial activation and accumulation at this late time point indicates an extended disease process within the retina ${ }^{228}$, and one that is unique when 

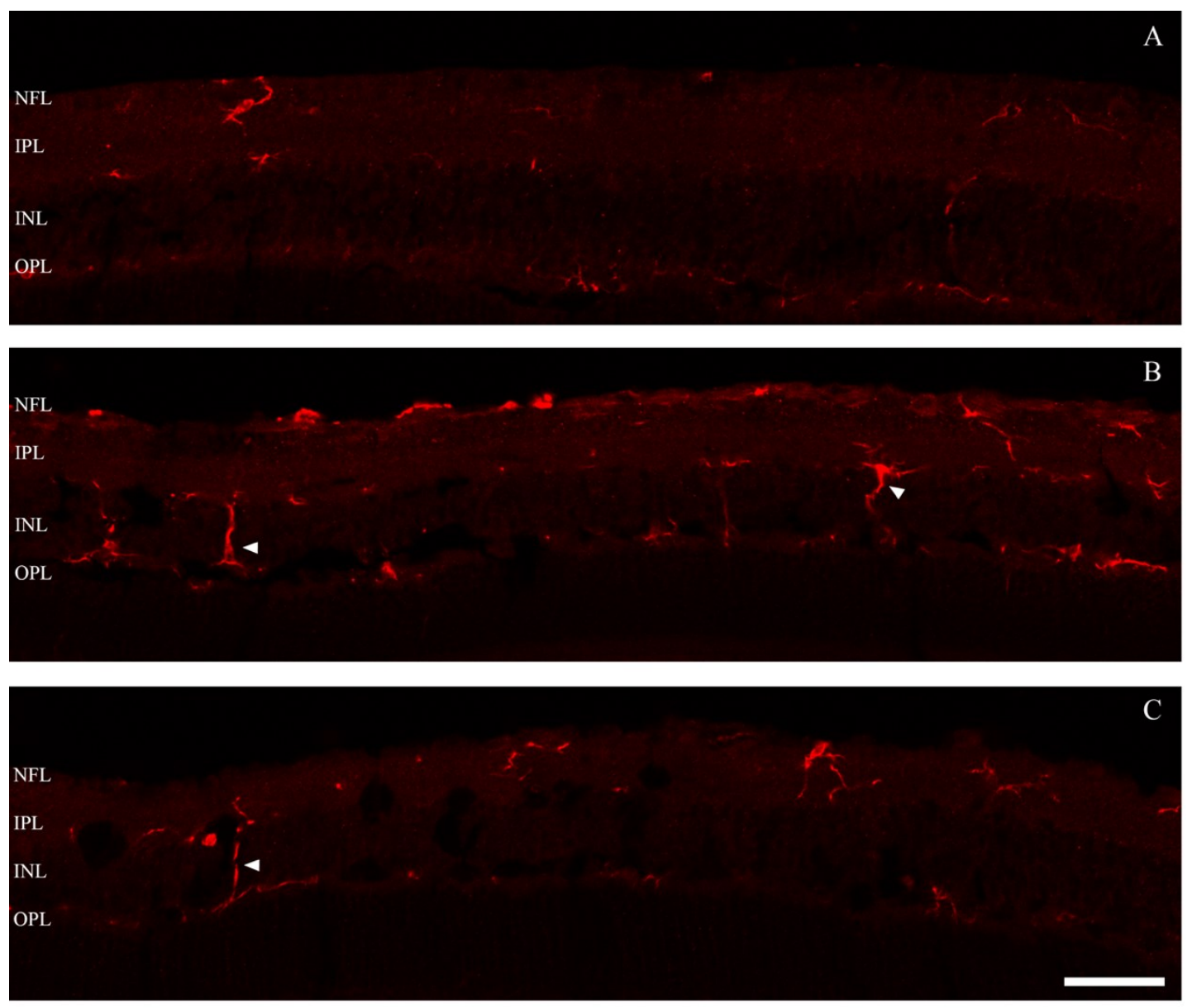

Figure 6-1. Microglia in the retina at 3 days after blast.

These images show Iba-+ microglia in the left retinas of $0 \mathrm{~V}(\mathrm{~A}), 50 \mathrm{~V}(\mathrm{~B})$, and $50 \mathrm{SMM}$ (C) animals at 3 days after blast. In $50 \mathrm{~V}$ animals, microglia appeared to penetrate further into the outer layers of the retina when compared to control eyes, and their processes seemed more numerous throughout the whole of the retina. Microglia visible in the $50 \mathrm{~V}$ animals seemed to have thicker processes, possibly indicating a reactive morphology. Drug treated animals had fewer microglia and fewer processes, but still penetrated into the more superficial layers of the retina. Both 50V and 50SMM animals had a large number of vertically oriented microglia, which appeared to reach across the retinal layers (white arrowheads). These were not evident in $0 \mathrm{~V}$ animals. The scalebar is $50 \mu \mathrm{m}$. 

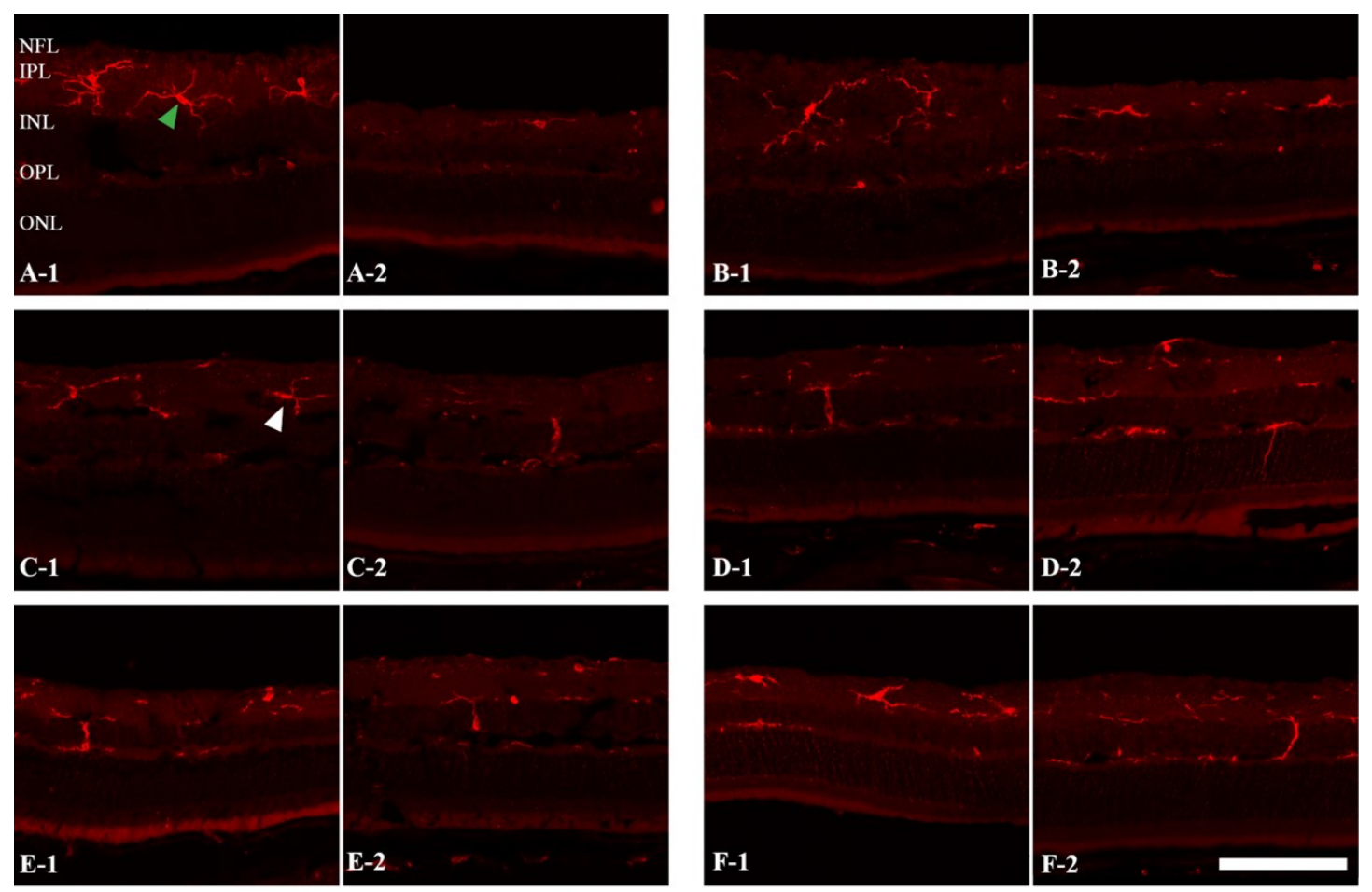

\section{Figure 6-2. Microglial morphology in the retina at 30 days after blast.}

These panels are from the eyes of $0 \mathrm{~V}$ (left eye A, right eye B), $50 \mathrm{~V}$ (left eye C, right eye D), and 50SMM (left eye E, right eye F) animals at 30 days after blast with Iba-1 immunostaining to visualize microglia. Panels 1 and 2 show two distinct cell populations seen during analysis. The control retinas had distinctive stellate microglia present in the IPL (A-1, green arrowhead). These were found in both the left and right eyes (A-1 and B-1, respectively). A majority of the cells were horizontally- oriented and within the inner layers of the retina in both eyes (A-2 and B-2). Blasted animals showed many vertically-oriented in both eyes (C-2, D-1, and D- 2), with some penetrating into the outer retinal layers (D-2). More horizontally-oriented cells in the left eyes of 50V animals had a characteristic lightening bolt appearance (C-1, white arrowhead), that were unlike those seen in controls. Drug treated animals had numerous vertically-oriented reaching cells in both eyes as well (E-1, E-2, and F-2), with fewer penetrating into the outer retinal layers than the $50 \mathrm{~V}$ animals. The horizontally- oriented cells within the inner retina of the left eye in 50SMM animals generally had a lightening bolt appearance similar to the $50 \mathrm{~V}$ animals. However, the right eye of 50SMM animals contained cells with thicker processes that did not have the characteristic lightening bolt appearance, but instead appeared to branch in all directions (F-1). The scalebar is $100 \mu \mathrm{m}$. 
compared to temporal patterns of microglial activation in the brain. Previously published experiments using this model found significant microglial activation in the OT beginning at 3 days and lasting until around 7 days after blast ${ }^{179}$. At and beyond 2 weeks postblast, no evidence of microglial activation was seen in any visual area, and yet evidence of actively degenerating axons were still found in both the optic nerve and the optic tract. The time period of microglial activation in the optic tract follows the temporal progression of axon bulbs and, subsequently, visible axon degeneration fragments, indicating their probable role of phagocytosing and disposing of axon degeneration products. But their quiescence at later time points when active degeneration is still occurring is puzzling. This may mean that brain microglia only respond in significant ways to a large number of axons degenerating, while fewer, scattered degenerating axons don't require microglial debris clearing. It could also indicate that the initial axonal degeneration was driven by a different mechanism, i.e. that of traumatic axonal injury, whereas later degeneration occurs as a result of the actual RGC cell dying, with the axon subsequently degenerating as well. If either of these is the case, the continued presence of reactive microglia in the retina at one month indicates the persistent need for phagocytosis and inflammatory regulation that is above and beyond what is necessary in response to axonal degeneration at this same time point in other areas. This is highly suggestive ${ }^{229}$ of ongoing cellular loss within the retina at this later time, which necessitates the continued activation of the resident microglia.

Müller glial cell response and retinal ganglion cell survival. Müller glia are an important support cell in the retina, releasing trophic factors, clearing away debris, and acting as a structural framework for neural RGCs ${ }^{230}$. Under pathologic conditions, Müller glial cells react to stress and increase GFAP expression in their processes. Normally, GFAP immunostaining is found predominantly in the NFL and RGC layer of the retina, but during pathology GFAP immunoreactivity will extend into the outer retina 231 , with the extent of penetrance being roughly correlated with the severity of retinal injury ${ }^{232}$. GFAP immunopositivity can be evident for long periods of time in ongoing disease processes, and can be an early indicator of retinal injury when few other signs are evident ${ }^{233}$. In focal retinal injury, GFAP reactivity can be seen as early as 24 hours, but in diffuse retinal injuries GFAP reactivity can take 7 days or longer to be visible ${ }^{234}$. Since there was no reason to think the retinal injury in this model was focal in nature, it was decided to examine GFAP immunohistochemistry at 7 days after blast, but not earlier. No increase in reactivity was seen in either eye of the $50 \mathrm{~V}$ or $50 \mathrm{SMM}$ animals at this time point when compared to controls. However, at 30 days post-blast, $50 \mathrm{~V}$ animals had a large increase in the number of GFAP processes in the LE retina compared to the LE of both $0 \mathrm{~V}$ and $50 \mathrm{SMM}$ animals (Figure 6-3A, C, and E). The right eye of $50 \mathrm{~V}$ animals showed a moderate, but non-significant increase in staining as well, but clearly had a slightly thicker zone of GFAP staining in the NFL at some areas of the retina, whereas other areas appeared the same as control animals (Figure 6-3B, D, and F). Widening of the NFL GFAP band is a very early Müller glial change ${ }^{235}$, possibly indicating that even earlier time points than those examined may show a similar activation pattern as the LE in $50 \mathrm{~V}$ animals. 

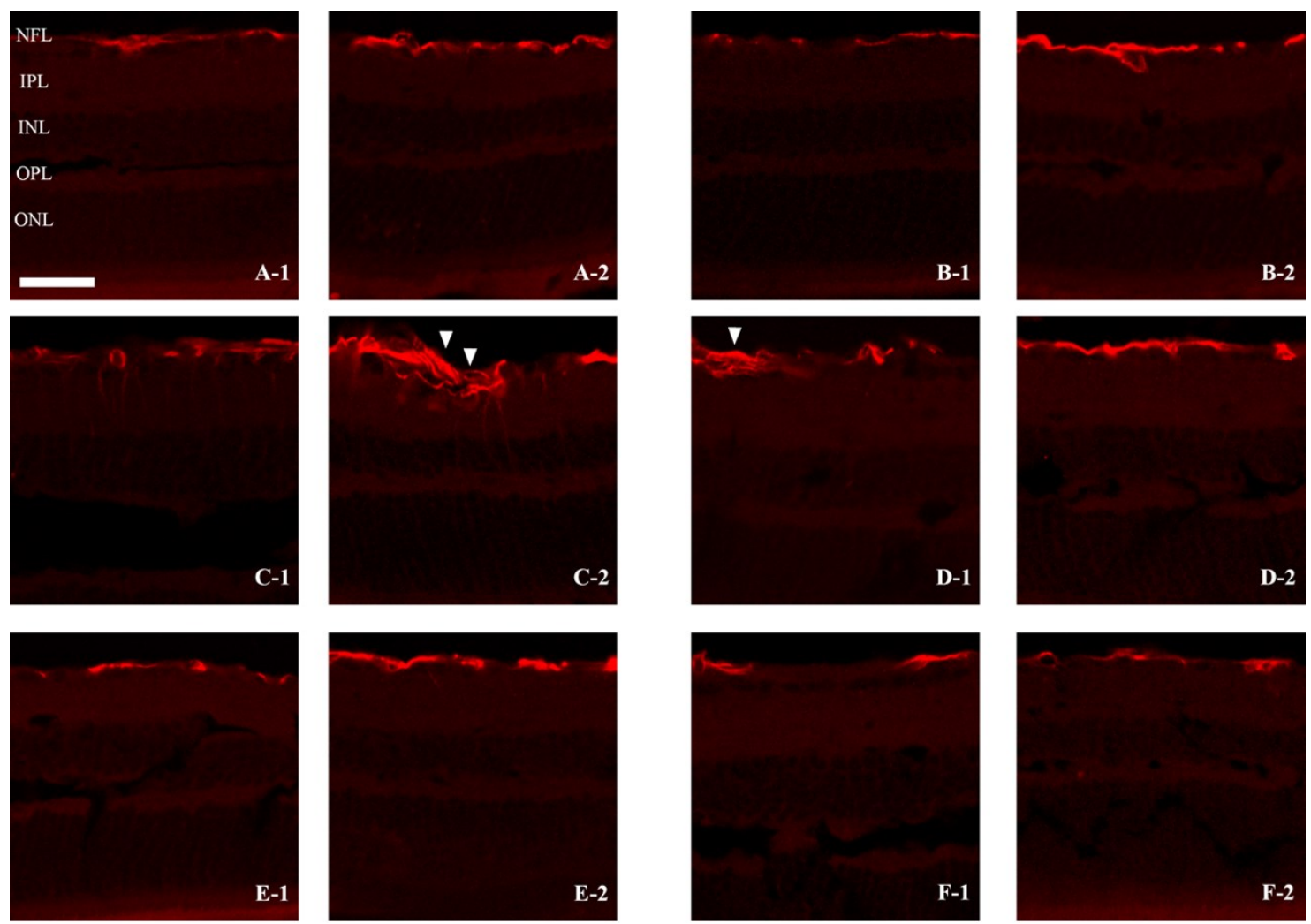

Figure 6-3. Müller glial morphology in the retina at 30 days after blast.

GFAP immunostaining was used to visualize Müller glial cell reactivity in the retina at 30 days after blast in $0 \mathrm{~V}$ (left eye A, right eye $\mathrm{B}$ ), $50 \mathrm{~V}$ (left eye $\mathrm{C}$, right eye $\mathrm{D}$ ), and 50SMM (left eye E, right eye F) animals. Panels 1 and 2 show different representative areas of the retina. The control animals showed a thin band of GFAP staining in the NFL of the retina in both eyes (A-1, A-2, and B-2), and occasional regions with little or no staining at all (B-1). 50V animals had a large number of processes in the left eye that penetrated into the IPL and beyond (C-1 and C-2), with a variably thickened band of GFAP staining in the NFL (C-2, white arrowheads). The right eye of 50V animals had much fewer GFAP processes that penetrated into the IPL, but still had a variable presence of NFL widening (D-1, white arrowhead). The 50SMM animals looked similar to control animals in both eyes, with little or no penetration of Müller cell processes, and a thin, variably absent band of GFAP in the NFL of both eyes (E and F). The scalebar is $40 \mu \mathrm{m}$. 
GFAP immunostaining in the LE of 50V animals was not uniform, but showed a patchy, sparse distribution. Some regions would have numerous processes visible in the IPL and INL, whereas other areas would appear the same as control retinas. Studies by others have shown that regions of reactive Müller cell glia usually correspond to focal areas of injury at that location ${ }^{236}$. Diseases that affect the whole retina, as with diabetic or drug-induced retinopathy, usually present with uniform and widespread GFAP reactivity ${ }^{237}$, whereas age-related retinal degeneration is indicated by a patchy GFAP reactivity that is scattered throughout the retina ${ }^{232}$. The latter pattern is the most similar to that seen in 50V LE retinas, and is important because it may speak to the injury mechanism of the left eye.

As discussed in Chapter 1, axonal injury from TBI is characterized as diffuse, and results from random shearing of axons in white matter tracts throughout the brain, with subsequent axonal degeneration. Given the orientation of the optic nerve and tract, being basically perpendicular to the vector of the blast wave, these structures might be particularly vulnerable to shear stress from the blast wave, resulting in diffuse axonal injury selectively in the RGC axons coursing through these white matter tracts. It has long been known that RGC axonal injury, even in the absence of direct somal injury, may lead to RGC cell body damage and possible degeneration ${ }^{238}$. If the underlying pathology in the LE is indeed due to this mechanism, diffuse RGC axonal degeneration with subsequent injury in the RGC cell bodies themselves, then reactive GFAP processes may indicate areas with damaged or dying RGCs whose axons have undergone degeneration. This would explain the patchy, somewhat atypical GFAP staining pattern, as axons experiencing damage from the blast would theoretically be randomly scattered throughout the affected structures. With that being said, the logical question is whether RGCs are, in fact, lost in the LE. An antibody to Brn3a was used to quantify this, and the LE of $50 \mathrm{~V}$ animals did show a nearly significant decrease in number of RGCs. The right eye showed a very minimal decrease in RGC number as well, but was not significant.

To further consider the possible relationship between RGC loss and reactive Müller glia, a Pearson correlation was run on RGC counts and GFAP counts. This identified a small negative association between the two factors that was not significant. This correlation hints that these GFAP processes may be present to a greater extent in retinas with greater RGC loss, although more investigations would need to be done to say this with any certainty. Importantly, drug treated animals also had a very nearly significant decrease in the LE RGC count compared to controls, a fact that initially seems to rebut the possible Müller glia-RGC inverse correlation, since these retinas were not found to have an increase in GFAP reactivity. One possible explanation for this is that the initial RGC loss at 30 days is due solely to the direct axonal damage of the initial injury, and this RGC loss is irreversible even in drug-treated animals. In this scenario, drug-treated animals may have an increase in anti-inflammatory microglia that may act to promote a cessation of the continued immune response, in which case reactive Müller cells in $50 \mathrm{~V}$ animals may represent areas where a second wave of RGC loss is occurring due to the degenerative environment of the first wave of cell loss, a phenomenon that has been observed experimentally ${ }^{238}$. The time course over which retinal ganglion cells degenerate after their axons are lost is heavily dependent upon the mechanism of injury 
239 , and further studies will need to be undertaken to investigate RGC loss at time points further out from blast. It may be the case that this hypothesized second wave of RGC loss will be much more significant in $50 \mathrm{~V}$ animals, and that at later time points a drug benefit would then be evident.

Optical coherence tomography. Various measurements of retinal thickness were collected by OCT both prior to blast, and then again at 30 days after blast. In this way, changes within the same animal could be tracked. No pre-blast/post-blast differences were observed in any treatment group for the right eye. In the left eye, there was a significant thinning in the outer retina of the $50 \mathrm{~V}$ animals, compared to their preblast measurements. The outer retinal measurement corresponded to both the outer nuclear layer and the photoreceptor inner and outer segments, and its significant diminution in blasted animals indicates the loss of and/or injury to photoreceptors in the LE. This finding is significant since it is the first evidence for outer retinal damage in this model. Photoreceptor alterations are common in the traumatic retinal injury known as Commotio Retinae ${ }^{240}$, and have been seen in motor vehicle accidents without any head injury ${ }^{241}$. This indicates the possibility that photoreceptor changes early after trauma can occur due to head acceleration, and possibly blunt ocular trauma ${ }^{242}$. Previous studies in our model have thoroughly documented that limited head accelerations occur after 60-psi blasts, which are classified as sub-concussive according to various injury criterion scales ${ }^{243}$. These same studies with our model imaged the blast administration at high speed and found no direct blunt ocular trauma. However, it may be the case that the blast wave causes a ripple effect through the retina that yields similar mechanical disruption to that of high speed head acceleration, and thus at early time points manifests as photoreceptor alterations. As will be discussed later, there are additional implications for the presence of photoreceptor pathology, having to do with the ERG findings. These will be considered in the section on functional vision tests.

It is necessary to discuss the potential conflict these findings display when compared to previous data. As discussed in Chapter 2, this mouse model has been shown to result in a significant thinning in retina of the left eye at 10 weeks after blast, while the right eye showed significant thickening. The thinning in the LE was traced to decreases specifically within the INL and the photoreceptor layer. However, in our pre-blast postblast OCT measurements at slightly over 4 weeks, there was no significant thinning in the total retina, nor in the inner retina, but there was thinning of the outer retina. This discrepancy can reasonably be attributed to one of two things: either the majority of the INL degeneration occurs at time points later than one month, or edematous fluid causes an artificial increase in the apparent thickness of the entire retina at 4 weeks post-blast. It seems possible that either or even both of these explanations may give rise to the OCT findings at 30 days. The fact that edema would leave outer retina thickness measurements still significantly decreased might indicate that photoreceptor degeneration occurs on a more escalated time scale than that of the INL. Right eyes at 30 days showed no signs of thickening in any layer, which seems to indicate that the right eye thickening in animals at 10 weeks after blast must occur after the 30 day time point. It is still possible that the thickening seen in the right eye at 10 weeks is due to either edema or 
sclerosis, since neither option can be definitively ruled out on the basis of the OCT findings.

\section{The optic nerve}

Quantitative assessments: Axon counts. Optic nerve cross sections were examined at 11 weeks after blast and found to be significantly atrophic in the LE of 50V animals. Random counts throughout the 4 quadrants of the optic nerve also revealed a significant decrease in the axon density and total axon count in the LE of 50V animals when compared with controls (Figure 6-4). This shows that after blast, axonal damage observed acutely ultimately results in axonal degeneration of those fibers. Drug treatment did not save the LE atrophy or total axon counts, but it did increase the axon density toward normal. From these data, it seems that the $50 \mathrm{~V}$ animals have atrophic, less densely packed LE ONs than $0 \mathrm{~V}$ animals, while drug treatment somewhat rescues the axon density, but leaves a significant number of axons still terminally injured. In the right eye, no atrophy of the $\mathrm{ON}$ was seen, but there was still a significant decrease in the axon density of $50 \mathrm{~V}$ animals when compared to controls. Drug treatment rescued the axon density in the right eye, making them indistinguishable from the control nerves. These right eye changes, however, yielded no significant changes in total axon number for any group.

Interestingly, when examining the relationship of the axon density and the $\mathrm{ON}$ area in the left and right eyes, additional insights can be gained for between-group differences. Axon density gives an idea of what a specific field of view within the optic nerve looks like at any given area when compared with other ONs. In ONs with lower axon densities, each field of view contains fewer axons and more non-axonal space. Non-axonal space is usually composed of glial processes and/or glial cell bodies, and generally increases in disease or pathology, corresponding to a concurrent decrease in axonal density ${ }^{244}$. In the left eyes of control animals, there is a negative association between axon density and the $\mathrm{ON}$ area (Figure 6-5). Since one would expect control animals all to have a similar number of axons in the optic nerve, our observed inverse correlation of axon density and the $\mathrm{ON}$ area is as expected. It is a simple matter of space efficiency in that, if axons are more densely packed with less non-axonal space, the optic nerve does not need to be as large to accommodate a normal number of axons, whereas if axons are less densely packed, the nerve area will be larger to accommodate those same axons. In $50 \mathrm{~V}$ animals, however, this trend is almost completely ablated, and there is instead almost no correlation between the axon density and the ON area (almost flat regression line). In 50SMM LEs, the trend is completely reversed from $0 \mathrm{~V}$ animals, with a relatively positive correlation between the axon density and $\mathrm{ON}$ area. Essentially, optic nerves that have a greater concentration of axons are also larger, whereas those with fewer axons are smaller in 50SMM LEs. This may be indicative of the presence of reactive gliosis, since optic nerves seem to have to increase in size to accommodate even densely packed axons. The right eyes of all groups show negative association trends similar to those of the 0V LE group, indicating that there is a different pathological mechanism underlying the damage in the right eye of blasted animals. The main 

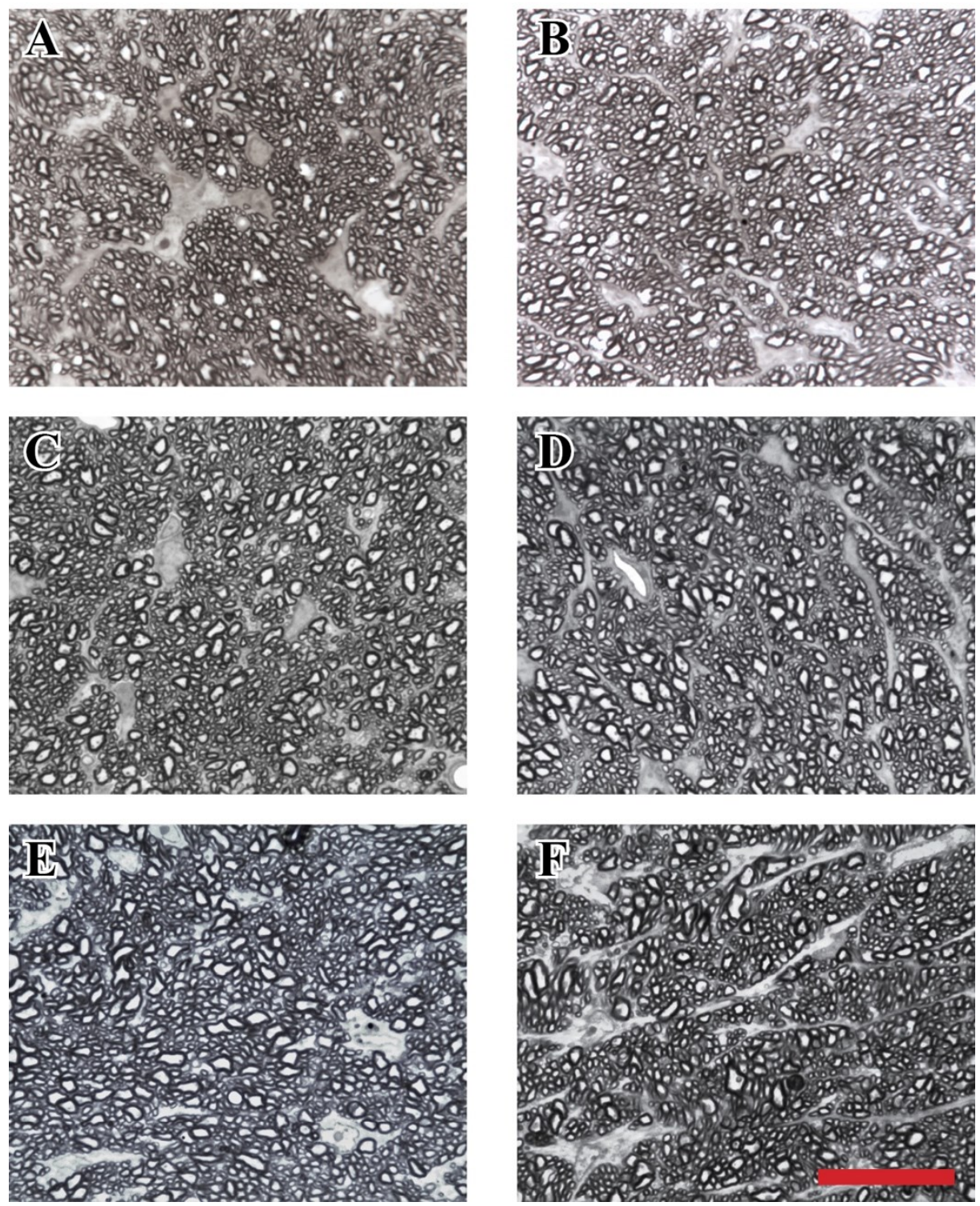

Figure 6-4. High power cross sections through the optic nerve to visualize and quantify axons at 11 weeks after blast.

This figure shows high power light micrographs through the optic nerves of $0 \mathrm{~V}$ (left eye A, right eye B), 50V (left eye C, right eye D), and 50SMM (left eye E, right eye F) animals. Even at this high magnification, no gross pathology is evident in either eye of $50 \mathrm{~V}$ animals (C and D), when compared to control animals ( $\mathrm{A}$ and $\mathrm{B}$ ). For this reason, manual axon counts were performed to investigate possible loss of axons. The scalebar is $20 \mu \mathrm{m}$. 


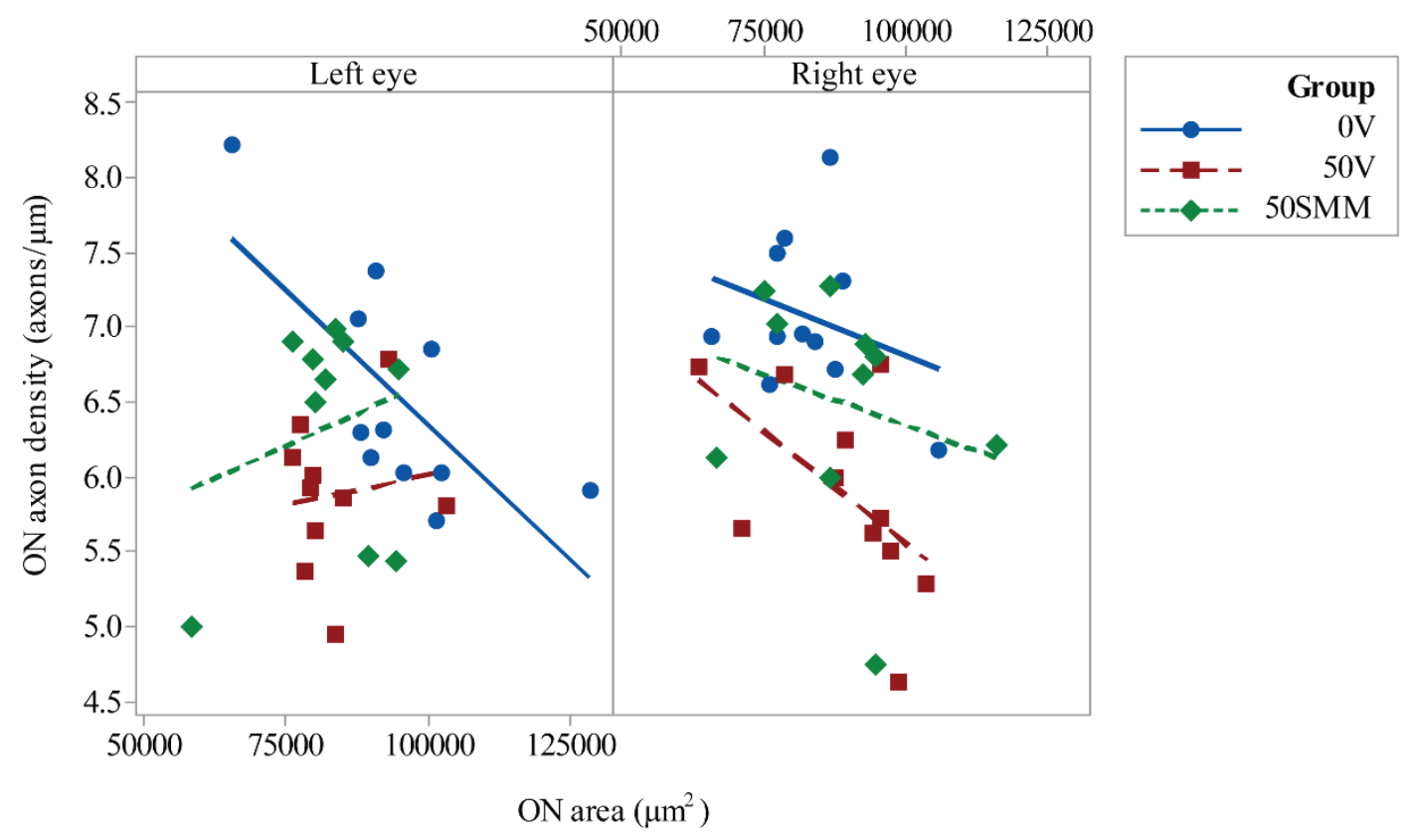

Figure 6-5. Scatterplot of optic nerve axon density versus optic nerve cross sectional area at 11 weeks after blast.

This figure shows a scatterplot to aid in visualizing the relationship between the $\mathrm{ON}$ axon density and the cross sectional area of these animals. A trend line is present for each group. In their left eye, control animals show a clear negative association between the axon density and the ON size. This makes sense, because more densely packed axons would not need as much ON size to accommodate them. The same trends hold true for the right eye of control animals. However, $50 \mathrm{~V}$ animals show a near ablation of the trend in the left eye, indicating that the axons are being lost and possibly replaced by glial scar of varying thicknesses, making the axon density now independent of the ON size. The drug treated animals have a reversal of the left eye trend seen in the $0 \mathrm{~V}$ animals, possibly also speaking to a reactive gliosis that is instead protective in nature. The right eyes of all groups show negative associations between the axon density and ON size measurements, showing that the pathology and presumably pathogenic process in this eye is different than that of the left eye. 
difference between groups for the right eye seems to be predominantly in the amplitude of the association, with $50 \mathrm{~V}$ animals showing a much lower range of axon densities than the $0 \mathrm{~V}$ group. The 50SMM group is increased toward normal, falling directly between the $0 \mathrm{~V}$ and $50 \mathrm{~V}$ groups.

Qualitative assessments from IHC. Analysis of the optic nerve using immunohistochemistry was difficult since so few ideal sections were captured through the optic nerve longitudinally in any one case. For this reason, the numbers of nerves available for examination were too small to use for quantification purposes. Nonetheless, some of the nerves offered important insight into the injury of $50 \mathrm{~V}$ animals that was not previously known, and for that reason it is important to discuss these specifics from a qualitative standpoint. The first significant finding was in one 50V LE optic nerve at 3 days after blast. This EYFP reporter mouse had numerous large axon bulbs clearly visible in the optic nerve just centimeters from the optic nerve head (Figure 6-6). Double labeling with Iba-1 showed these bulbs to be associated with a population of large reactive microglia, appearing more amoeboid than in any other areas of the brain or visual system. Previously, it was not known whether axonal injury was localized to any specific area along the axons running from the eye to the optic tract in the brain. Given the abnormally large number and size of the bulbs found in this case, especially when compared to the axon bulbs found in the optic tract of this same animal, it is hypothesized that the injury in the optic axons originates from an injury more proximal to the eye, possibly at a point of torsion where the optic nerve runs through the bony optic nerve canal in the skull, yielding a significant point of stress ${ }^{245,246}$ (Figure 6-7).

GFAP immunostaining also gave an interesting case study in LE ONs at 30 days after blast. The astroglia form a structural support mosaic in the ON distal to the eye, and are highly ordered in the myelinated regions of the optic nerve ${ }^{247}$. Like in the retina, this meshwork can give insights into reactive changes occurring during pathology. In crush injuries of the optic nerve, GFAP meshwork forms gliotic scarring that is clearly visible localized to the site of injury ${ }^{248}$. Likewise, examining the left $\mathrm{ON}$ of a $50 \mathrm{~V}$ animal immunostained for GFAP, we were able to identify an obvious increase in the GFAP network along one side of the optic nerve that seems to localize around two blood vessels (Figure 6-8). In this case, it is obvious that the GFAP meshwork has an altered orientation from a uniform cross-hatch pattern, to a more radial and clearly asymmetric pattern of orientation. Again, these data could not be quantitatively analyzed, but give interesting insight into the injury in this model. Also, it is important to note that no 50SMM animal displayed this type of asymmetric astroglial change within an individual optic nerve, indicating that the drug improved, or at least decreased this injury response.

\section{The optic tract}

The optic tract is the posterior continuation of the axons originating from the RGCs in the retina. As they leave the eye in the optic nerve, the fibers decussate at the optic chiasm, where in the mouse a large majority of fibers from the left eye cross over

and continue on the contralateral side ${ }^{249}$. Our previous work had shown indications of 


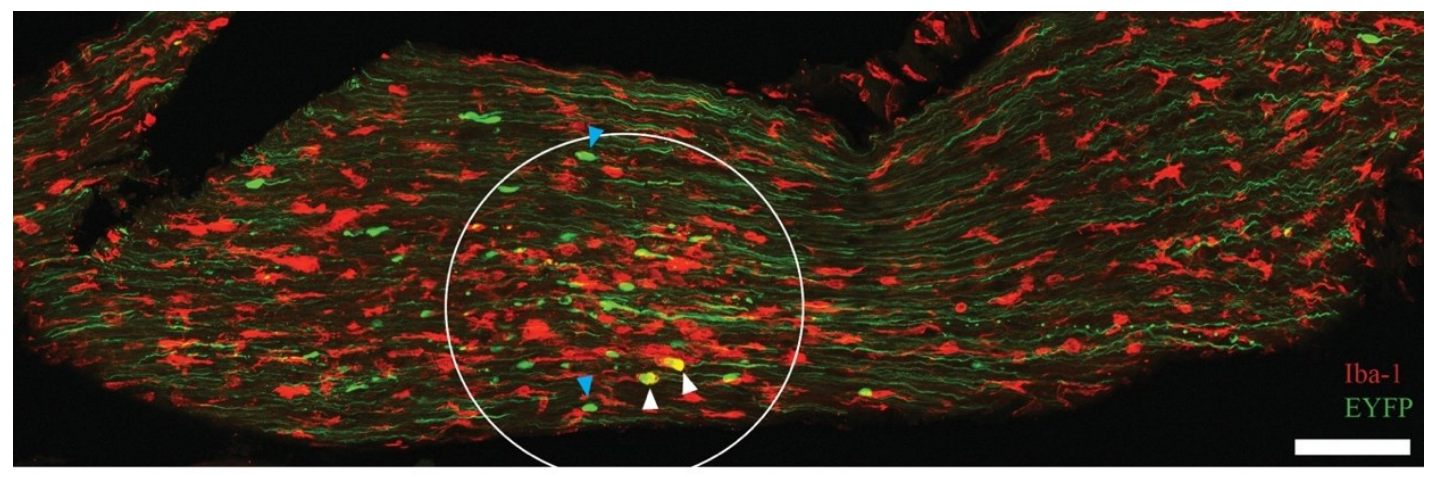

Figure 6-6. Histologic evidence for a focal optic nerve injury in a 3 day post-blast animal.

This figure shows a longitudinal section through the proximal left optic nerve of a $50 \mathrm{~V}$ animal 3 days after blast. Double labeling for EYFP and Iba-1 allows visualization of both axons (green) and microglia (red) in the same section. Within the white circle, an intense concentration of large diameter axon bulbs (blue arrowheads) is present, surrounded by intensely-stained, amoeboid microglia, some of which are seen engulfing axon bulbs (white arrowheads). This focalized area of injury may indicate a point of torsional stress on the left $\mathrm{ON}$ during blast, as it is held stationary passing through the bony canal of the skull to leave the orbit, while both the brain and eye remain relatively mobile. The scalebar is $100 \mu \mathrm{m}$. 


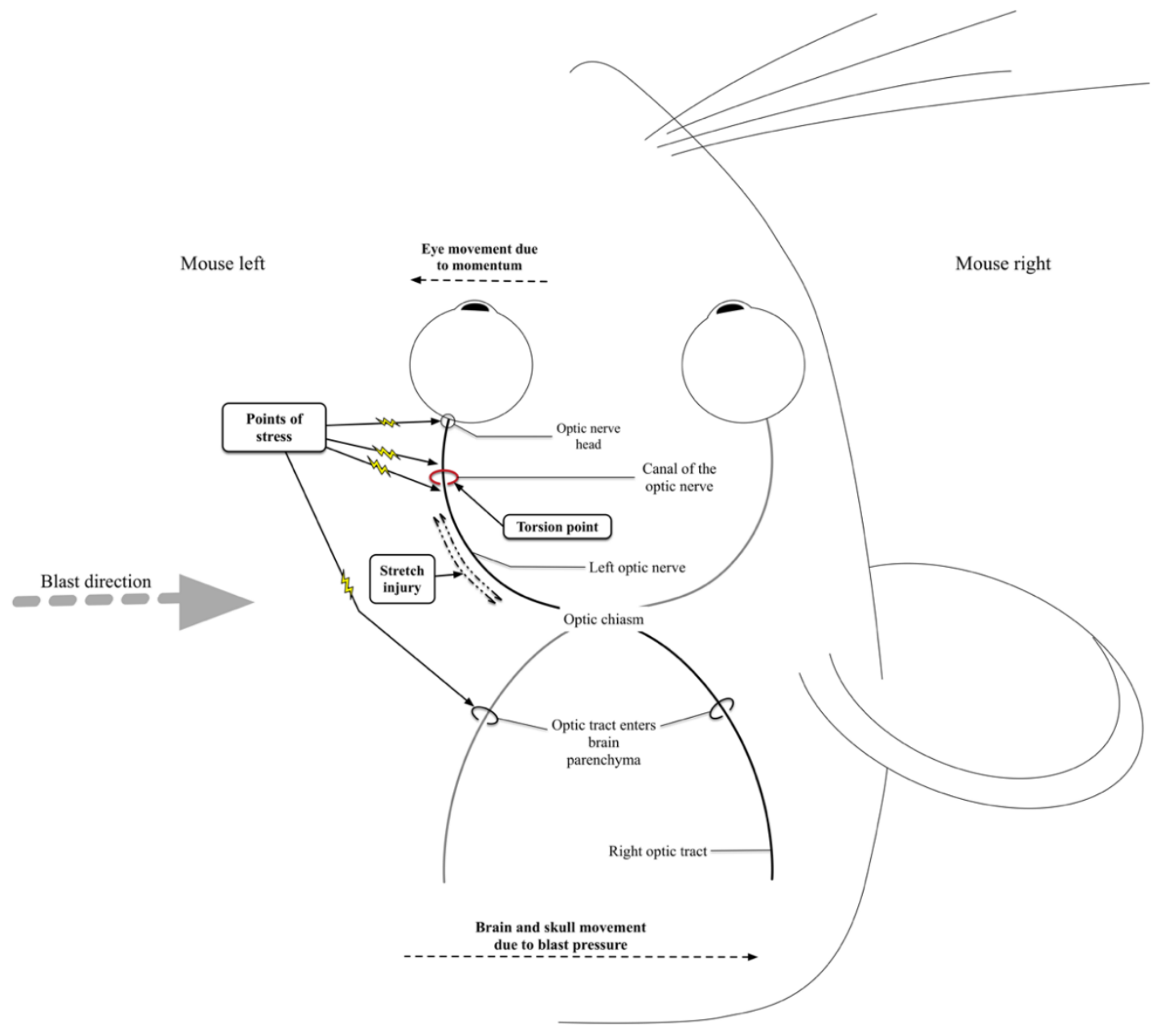

Figure 6-7. Hypothesized left eye injury mechanics in the moments directly after blast.

This schematic shows a top-down view of the mouse's head and visual system, along with hypothesized mechanics that may occur in the left eye moments after blast. The blast wave, coming in from the left, causes movement of the head (and brain) toward the right. The eye, having more degrees of freedom, would move left relative to the head, due to momentum. In this way, the canal of the optic nerve would serve as a point of torsion, where the optic nerve head, the optic nerve on either side of the bony canal, and possibly the optic tract as it enters the brain, would feel shear stress leading to mechanical injury. This type of movement would also cause normal stress in the axons of the left optic nerve, leading to an axonal stretch injury. As discussed in the text, the right eye injury may stem from a combination of central visual damage and sympathetic ophthalmia from the LE inflammatory response. 

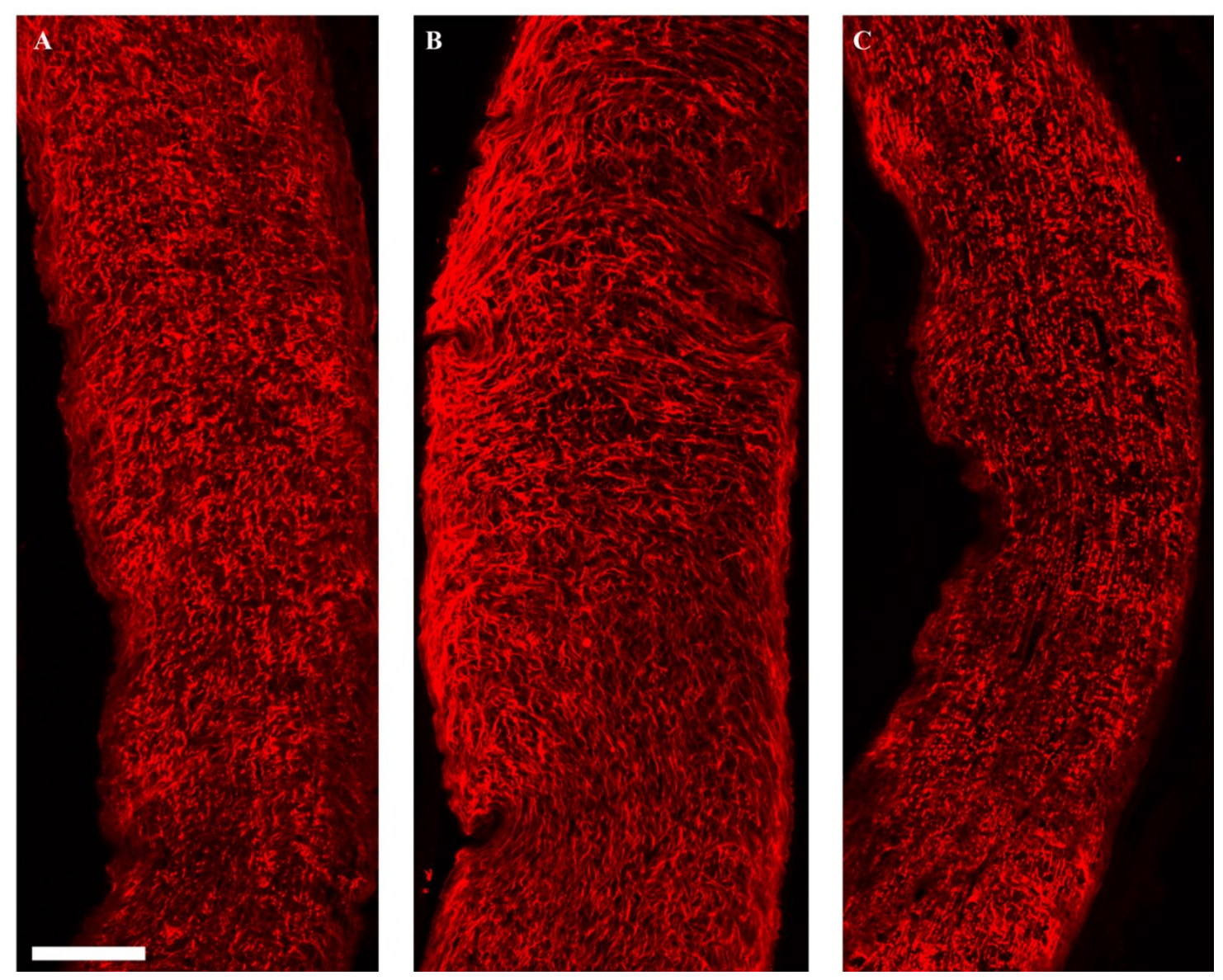

Figure 6-8. Alterations in the glial meshwork of the left optic nerve at 30 days after blast.

This figure shows the glial meshwork of the left optic nerve at 30 days after blast in longitudinal sections immunostained with GFAP. The $0 \mathrm{~V}$ animal (A) shows a somewhat random appearing meshwork with no apparent densely stained or ordered areas, consistent with the normal structural framework of the $\mathrm{ON}$. In the $50 \mathrm{~V}$ animal (B), there is an obvious confluence of glial processes on the leftmost border of the optic nerve, appearing to encircle what may be small blood vessels. This localized organization of glial processes indicates a focalized injury with subsequent glial scarring, such as may occur at a point of torsional stress to the optic nerve as it courses through its bony canal to leave the orbit. The drug treated animal (C) shows no such ordering of glial processes. The scalebar is $100 \mu \mathrm{m}$. 
axonal disruption in the right optic tract (ROT), and to a much less extent in the LOT, in the form of axon bulbs. It was not known in this model whether the axonal injury occurred as a result of the primary mechanical insult, or instead as a secondary injury caused by immune and glial over-activation, preventing even minimally harmed axons from regenerating ${ }^{250}$. Because of the high lateralization of the axon injury, it was decided to selectively investigate the ROT in these experiments. The animals were examined at 3, 5, and 7 days after blast. Additionally, drug treated animals were examined at 3 days for comparison with $50 \mathrm{~V}$ animals at this acute time point.

Axonal injury. At 3 days after blast, bulbs in 50V animals were large, and scattered randomly throughout the optic tract, exhibiting a heterogenous size distribution from 6.5-25.6 $\mu \mathrm{m}$ (Figure 6-9). At 5 days after blast, the majority of bulbs in $50 \mathrm{~V}$ animals began to shrink in size, and take on a more granular appearance. Finally, by 7 days, very few large bulbs were evident, but there were numerous dotted and granular bulbs visible throughout the whole ROT. This pattern is highly consistent with the morphology of degenerating axons undergoing Wallerian degeneration secondary to traumatic axonal transport disruption ${ }^{251}$. As discussed in Chapter 2, disruption of axonal transport occurs secondary to microtubule disruption, causing axolemmal varicosities where cellular transport products build up. This can be due to traumatic mechanical insult, or other pathologic processes, and can result in axonal degeneration ${ }^{252}$. In vivo investigations of axon lesions have shown that, proximal to the site of injury, axon bulbs can be sometimes seen within hours, while the portion of the axon distal to the injury may not show signs of morphologic change until as long as 36 hours afterward ${ }^{253}$. Following this temporary delay, the distal axon undergoes a rapid axolemmal segmentation often known as blebbing, which aids in subsequent cleanup by phagocytic cells ${ }^{254}$. Occasionally, portions of the axon proximal to the site of injury can also undergo blebbing, which can variably affect the soma of the cell depending on its proximity to the injury ${ }^{253}$. This time course and morphologic transition indicates that the bulbs initially seen in these animals at 3 days after blast probably represent a distinctly different phenomenon than those seen at 5 and 7 days after blast. For this reason, the size of the bulbs included in the quantification were restricted to only those larger than 6.5 $\mu \mathrm{m}$, since bulbs smaller than this became difficult to distinguish from axons diving in and out of the section, as well as small axon blebs. Bulb counts in $0 \mathrm{~V}$ animals at 3, 5 and 7 days found few, if any bulbs present. In $50 \mathrm{~V}$ animals, there were significantly more found at all time points, with a peak number seen at 3 days and tapering off until 7 days. In our previous studies, no bulbs were found in the OT at any time point examined later than 7 days. Drug-treated animals examined at 3 days after blast had a near-significant decrease in the number of bulbs compared to their $50 \mathrm{~V}$ counterparts, and were statistically equivalent with $0 \mathrm{~V}$ animals. These results indicate that much of the axonal degeneration seen at 3 days in this model can be rescued by abating immune-mediated secondary injury.

The M1/M2 microglia story. Our evidence suggests that an integral part of the SMM-189 benefit in this model was the role of differentially polarized microglia, and their regulation of immune function after trauma. Previous studies have indicated that SMM-189 may work by altering the polarization of activated microglia from a pro- 

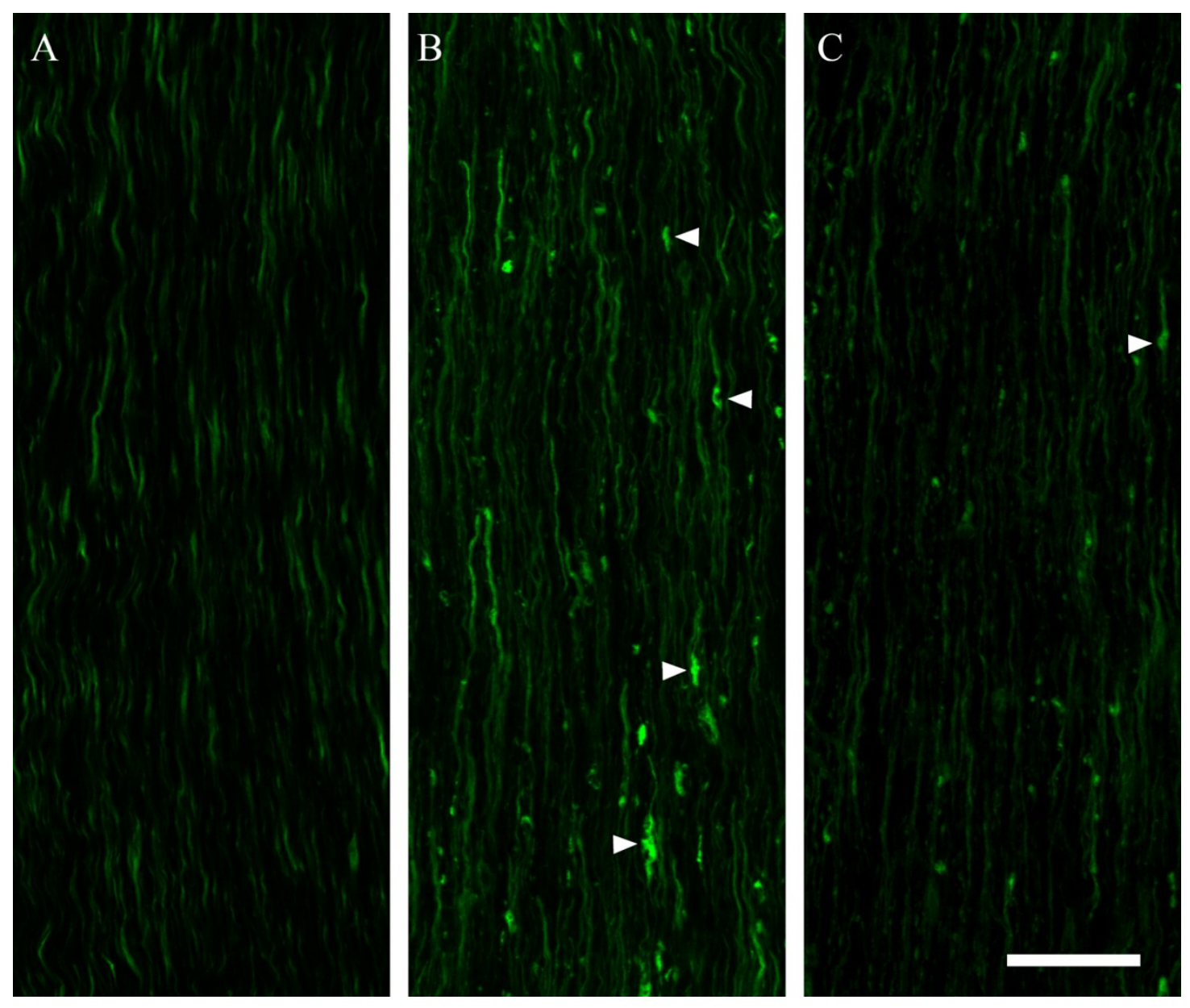

\section{Figure 6-9. Axon bulbs in the right optic tract at 3 days post-blast.}

This figure shows longitudinal sections through the right optic tract of EYFP reporter mice at 3 days after blast. In $0 \mathrm{~V}$ animals (A), the axons can be seen diving in and out of the section, are all relatively uniform in size, and contain no obvious regions of axolemmal disruption. The $50 \mathrm{~V}$ animals (B), however, show a large number of EYFP accumulations, and frank axon bulbs (white arrowheads), indicative of areas of traumatic axonal transport disruption. Drug treated animals (C) have significantly fewer, but still evident bulbs (white arrowhead) at this time point. The scalebar is $50 \mu \mathrm{m}$. 
inflammatory M1 state to a neuroprotective M2 state. These different microglial states are commonly identified by distinct cell surface markers CD16/CD32 (M1), and CD206 (M2) ${ }^{131}$. CD16 and 32 are low affinity Fc receptors, which bind to the Fc portion of antibodies and assist phagocytes in ingesting opsonized cells or bacteria ${ }^{255}$. CD206 is known as the mannose receptor, and is present on cells to also assist with phagocytosis, but also seems to have important intracellular functions for cytokine expression ${ }^{256}$ and clearing lysosomal hydrolases during inflammation ${ }^{257}$. In order to determine whether SMM-189 was functioning in this model by way of microglial polarization shifts, IHC for these two markers was done in the ROT of animals at 3 days after blast. Unexpectedly, the two markers were expressed in nearly every Iba-1 positive cell in the OT of all groups, making a clear M1/M2 distinction impossible based on qualitative assessment alone (Figure 6-10). For this reason, it became necessary to classify microglia instead based on the marker that showed predominance on a cell-by-cell basis. We found that M1-predominant microglia comprised the majority of microglia in all groups, but to a much greater extent in the $50 \mathrm{~V}$ animals. Control animals had the highest number of microglia without a predominant marker, as would be expected for resting microglia. The M2-predominant cells were present in very low numbers in $50 \mathrm{~V}$ animals, and drug treatment increased the presence to a level almost equivalent to control animals. Examining the changes of each type of microglia across the three groups gives insight into how microglia alter their polarization in each microenvironment (Figure 6-11). When comparing $50 \mathrm{~V}$ animals to $0 \mathrm{~V}$ animals, it becomes apparent that there is an almost complete conversion of M0 and M2-predominant microglia into M1 microglia, increasing the M1 count by almost $40 \%$. This makes sense, as one would expect the microenvironment during trauma to selectively polarize microglia into the M1 state in response to inflammatory signals. With drug treatment, there is a $20 \%$ decrease in the number of M1 reactive microglia, with almost $100 \%$ of these converted into M2 microglia when compared to $50 \mathrm{~V}$ animals. This change was significant across all groups, supporting the view that the drug does act on at least part of the microglial population to polarize them into a more protective phenotype. Importantly, there is also a negative correlation between the number of axon bulbs and the number of M2-predominant microglia in the same section of ROT in SMM-treated animals, although this correlation did not reach significance (Figure 6-12). This hints that this increased presence of M2 microglia may be protective against bulb formation.

The classical view of microglia as having distinct polarization states has long been understood to be an oversimplification, but its usefulness in providing a simple nomenclature to fit with their role as being either pro- or anti-inflammatory in action has perpetuated its existence ${ }^{258}$. It was not until recently, with an increase in research focused on ways to target the immune system in neuropathology, that the oversimplification has posed a significant barrier for in vivo investigations ${ }^{259}$. The classical view defines specific cell surface markers as being selective for an M1 or M2 polarization state, however this was clearly not the case in our experiments. Almost all cells, even those with significantly greater staining for the M2 mannose receptor, had a level of CD16/32 expression higher than that of background, indicating at least a low level of co-expression for both markers. Other recent studies have also found a large number of cells co-expressing surface markers that are classically defined as being M1 

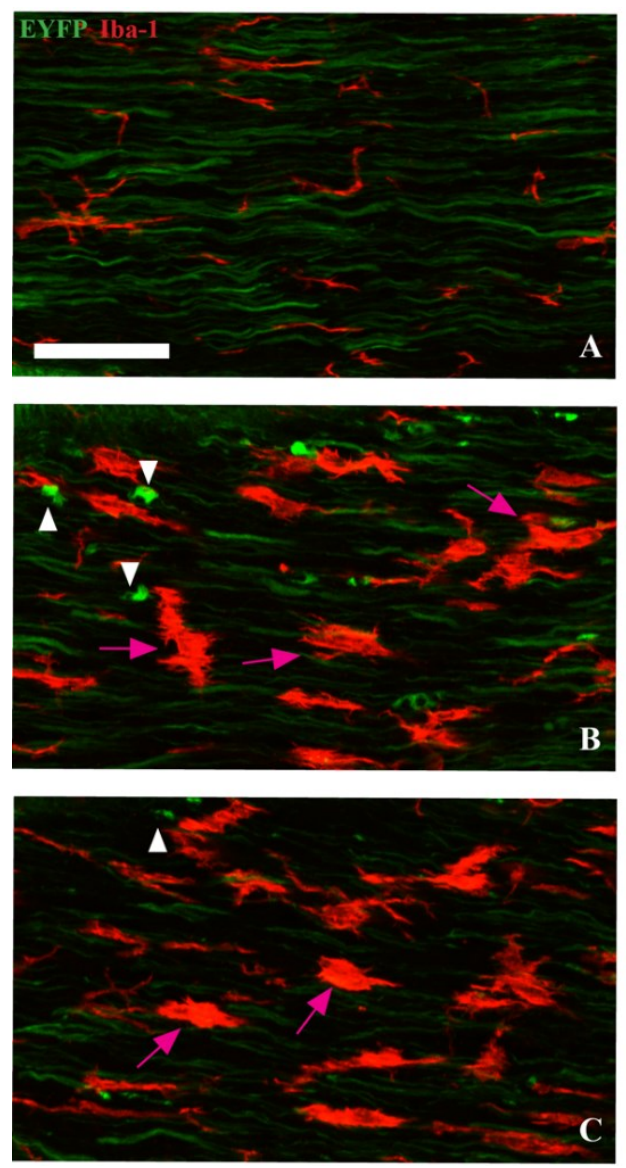
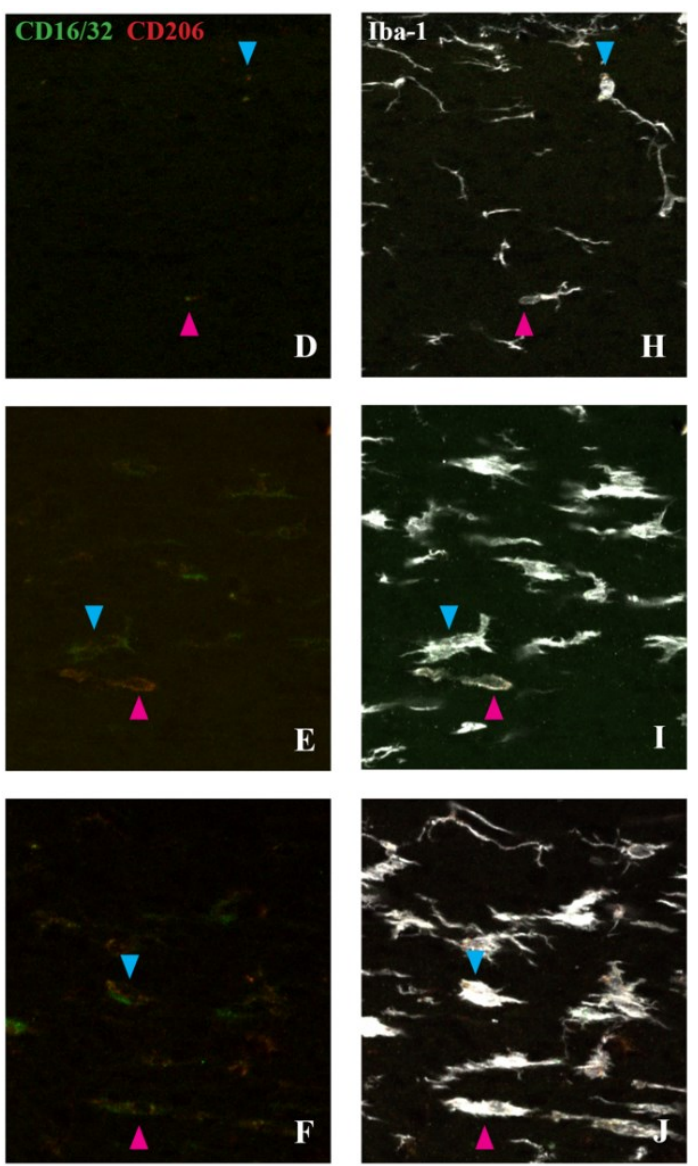

Figure 6-10. Microglial morphology and polarization markers in the right optic tract.

This figure shows double labeled cross sections through the right optic tract. Panels A, $\mathrm{B}$, and $\mathrm{C}$ show EYFP+ axons (green), and Iba-1+ microglia (red). Control microglia are delicate and highly arborized, indicative of resting microglia (A). The axons are ordered and uniform in size. In $50 \mathrm{~V}$ animals (B), microglia have retracted their processes, and are highly reactive in their morphology (pink arrows). Reactive microglia can be seen in close association with bright green axon bulbs (white arrowheads). Drug treated animals (C) show significantly fewer axon bulbs (white arrowhead), with some reactive (pink arrows), but also some resting microglia. Panels D-J show sections stained for either CD16/32 or CD206 (D, E, and F) or Iba-1 (H, I, and J). Iba-1 panels adjacent to CD16/32 or CD206 panels are the same field of view, where blue/pink arrowheads indicate the same cell in each panel. In the control animal, very little CD16/32 or CD206 staining is evident (D), although it is clearly staining microglial cells $(\mathrm{H})$. In $50 \mathrm{~V}$ animals, much greater staining is evident overall, with more CD16/32 present (E). 50SMM animals also have a high level of staining (F) compared to controls. From sections $\mathrm{E}$ and $\mathrm{F}$ it is apparent that M1 and M2 markers are highly colocalized, although varying in intensity in each cell. Panel E demonstrates a cell that clearly stains more predominantly for CD16/32 (blue arrowhead), where an adjacent cell stains more predominantly for CD206 (pink arrowhead). The scalebar is $50 \mu \mathrm{m}$. 


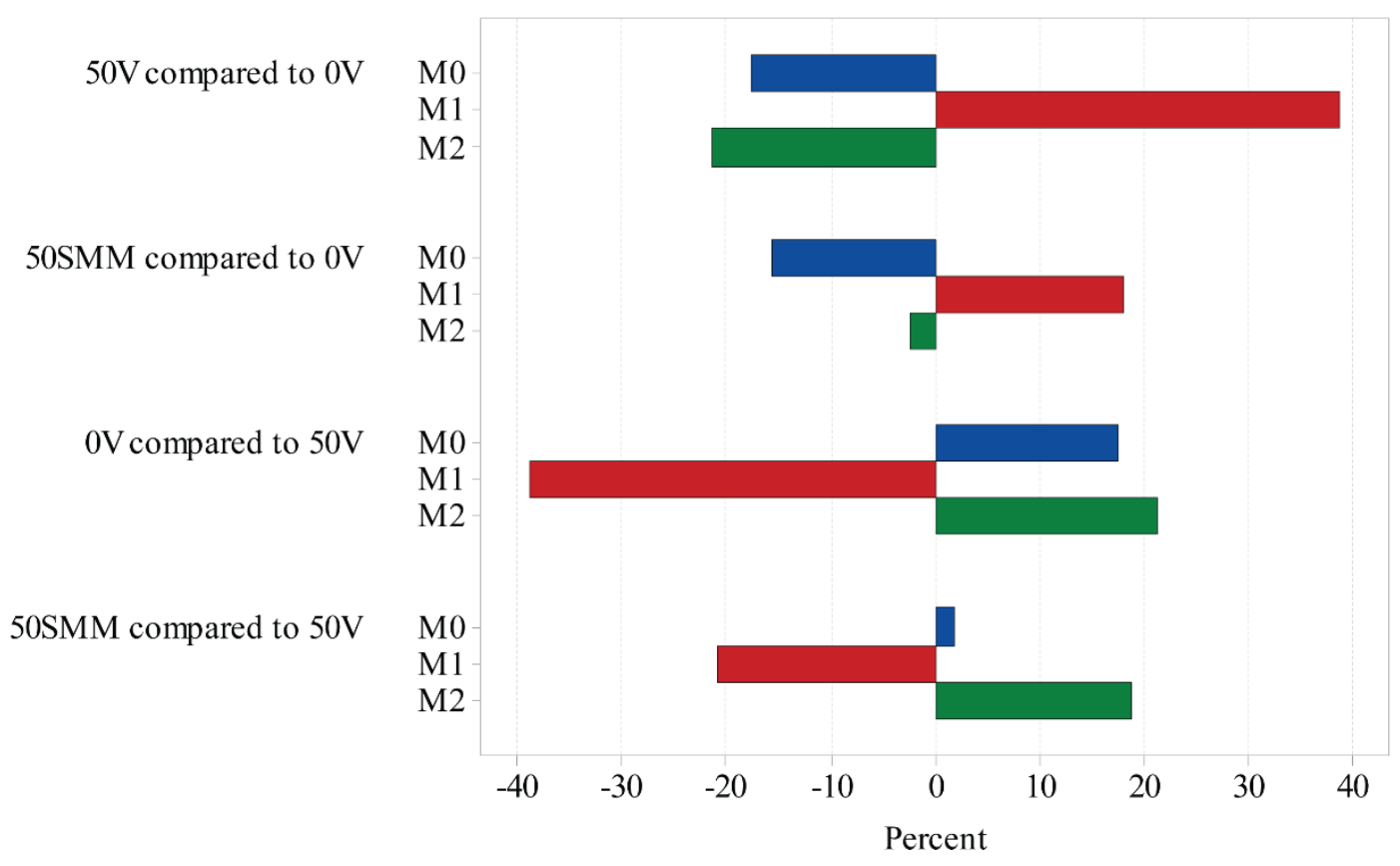

Figure 6-11. Microglial polarization comparison chart.

This comparison chart allows visualization of how microglial polarization is altered from one group to another. When comparing $50 \mathrm{~V}$ to control animals, it is clear that there is a conversion of both $\mathrm{M} 0$ and M2 microglia into M1 reactive microglia. 50SMM animals are more similar to $0 \mathrm{~V}$ in their microglial polarization profile than $50 \mathrm{~V}$ animals. When comparing 50SMM to 50V animals at the bottom of the chart, it is clear that there is almost a direct conversion of M1 reactive microglia into M2 microglia, lending support to the hypothesis that SMM-189 acts by altering microglial polarization. 


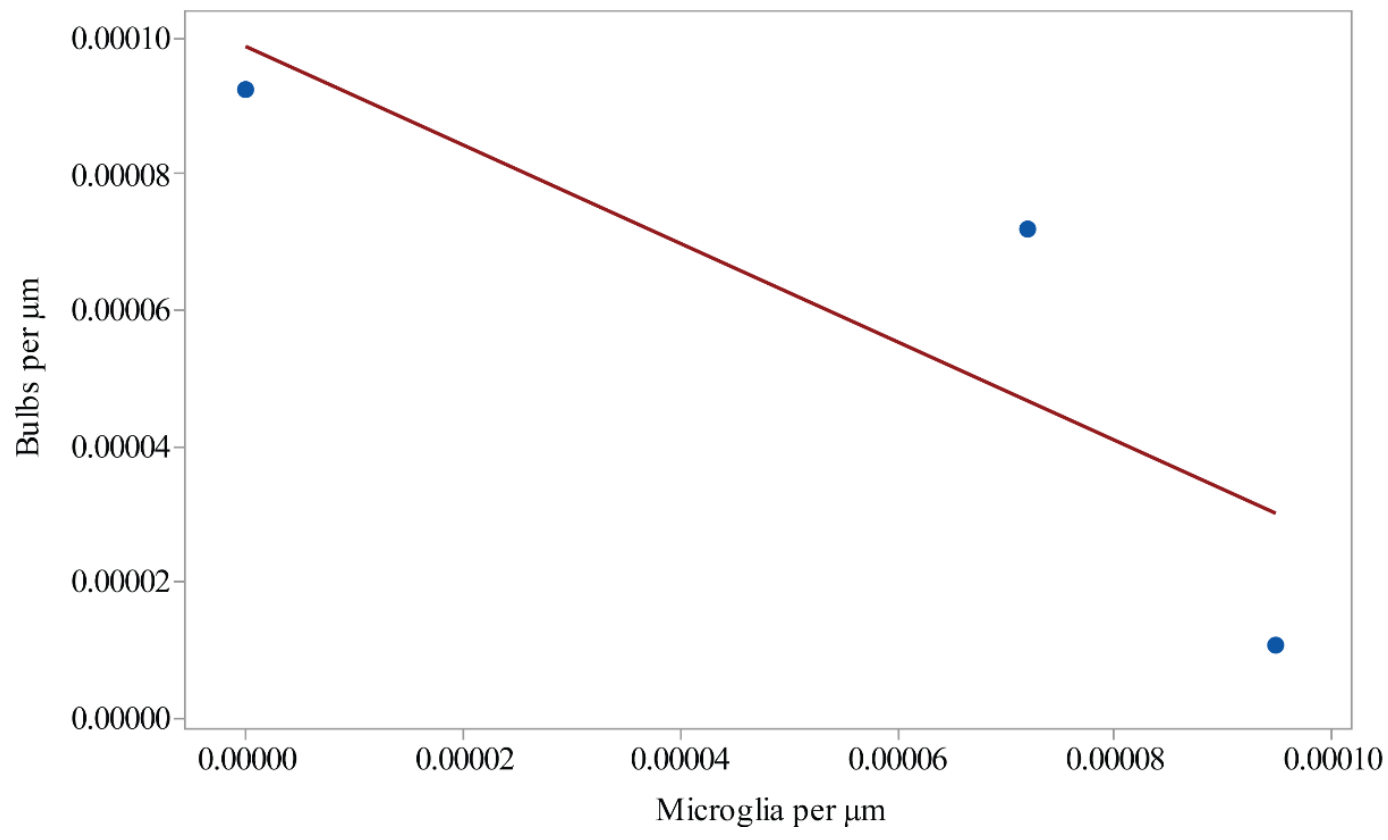

Figure 6-12. Scatterplot of axon bulbs versus number of M2-biased microglia.

This scatterplot shows the relatively strong negative correlation between the number of axon bulbs in the right OT and the number of M2-biased microglia. This correlation indicates a protective role for M2-biased microglia in helping to prevent axon bulb formation. 
and M2-specific. The expression levels in these experiments differed significantly and seemed to be dependent upon both disease progression and pathogenic mechanism at work ${ }^{260,261}$. Most experiments done defining microglial polarization have used cytokine and receptor expression profiles from cells stimulated with one or two specific cytokines 124. It is clear that in vivo, such unadulterated cytokine stimulation is probably never encountered, and instead many conflicting M1 or M2 signals may all exist concurrently. This line of reasoning has prompted many researchers to re-define the thinking on microglial polarization, instead positing that microglial polarization is a continuum, of which M1 and M2 represent extreme ends of this spectrum, and must be treated as such 258 .

In our study, it was somewhat surprising to find that control animals also had a large number of cells expressing both M1 and M2 markers. Many cells contained equal amounts of both markers, while a majority of cells expressed predominantly CD16/32, an M1 marker. One possible explanation for this is that these mice were not actually pure untouched controls, in that they were injected with a vehicle solution on the same regime as drug-treated animals. While the vehicle solution itself may not have had immuneinitiating actions, it is possible that a low-level systemic inflammation was initiated by ip injections in these animals. Systemic inflammation is known to play a critical role in CNS immune system activation ${ }^{262}$, and could have caused a brain-wide subclinical priming of microglia, subtly altering their surface receptor expression. Another explanation is that, as has been suggested by recent literature, even these "resting" microglia may still exhibit receptor expression levels normally attributed to an activated phenotype, in the absence of apparent reactive stimuli. One paper suggests that resting microglia might more closely resemble M2, neuroprotective microglia, phenotypically since they perform many of the same functions for normal tissue maintenance and immune system regulation ${ }^{263}$. Either way, it is clear that assuming the existence of a simple M1/M2 dichotomy might not be practical in this model, or any in vivo model hoping to deeply probe these questions. Future studies of the in vivo action of SMM-189 must address this issue in a more complete way, using numerous M1/M2 markers in combination to fully represent the full spectrum of microglia states present in any microenvironment.

\section{Functional Visual Tests and Their Relevance to Histologic Findings}

\section{The ERG B wave increase: Discussions of a Seemingly Paradoxical Finding}

In $50 \mathrm{~V}$ animals, the left eye showed a significant increase in the ERG B wave peak amplitude 30 days after blast, both compared to their pre-blast measurements, and when compared to control animals. The right eye showed a similar trend, but did not reach significance. The A-waves of both eyes showed a corresponding trend toward being higher in amplitude than the control animals, while also failing to achieve significance. There was no change in the latency time for any of the ERG components measured in any group. Drug treatment remedied this ERG defect, bringing both the A- 
and B-waves of both eyes back toward control levels. Under pathologic conditions, the ERG waves are almost universally expected to show a decrease in the peak amplitude of one or more components ${ }^{264}$. This makes sense, because the ERG wave is composed of summed voltage originating from retinal cellular activity. If retinal cells are damaged, it would be assumed that the electrical activity from these cells would decrease. Because of this, ERG wave increases due to pathology are rarely reported, rendering the ERG findings in these experiments to be somewhat paradoxical. Importantly, at least one other group has seen ERG wave augmentation in a blast overpressure-induced TBI model ${ }^{265}$, while clinicians working with human cases of TBI visual disturbance have also identified such anomalies ${ }^{266}$. Despite this, it seems that ERG wave augmentation remains poorly understood. Theoretically, under specific conditions, the ERG wave response might be altered by both intra- and extra-retinal changes, one or many of which may be at play in animals exposed to blast overpressure. In order to discuss these possibilities, it is necessary to first understand the components of the ERG wave, and what contributes to them.

Contributors to the ERG waveform: Evidence from the literature. Scotopic ERG readings acquired from the corneal surface represent a summation of radiallydirected currents emanating from the retina which pass through the vitreous and anterior portions of the eye, to finally pass through the cornea and sclera, and circle back around 215. The cells contributing to the A-wave component are the photoreceptors, where the negative A-wave corresponds to the hyperpolarizing current occurring when light interacts with a photoreceptor. For this reason, the A-wave is said to be a pre-synaptic response. The B-wave component is generally thought to be dependent upon Müller glia and bipolar cells, although this seems to be debated in the literature for reasons that will be discussed. Generally speaking, the B-wave represents the post-synaptic transmission of signal from the photoreceptors to second-order cells in the ONL. The C-wave is the final major component of the ERG, and is derived from RPE activity.

The specific cellular contributions to the B-wave component of the ERG are complex, and countless experiments have been done aimed at clarifying this information. One major hypothesis is that the B-wave originates solely from Müller glia, which generate a large current from selective uptake of light-evoked extracellular $\mathrm{K}^{+267}$. Lightevoked $\mathrm{K}^{+}$can come from ON-bipolar cells in the OPL and amacrine cells and RGCs in the IPL, and thus in this scenario B-wave currents are also indirectly dependent upon generation of those light-evoked $\left[\mathrm{K}^{+}\right]$increases ${ }^{267}$. However, experiments with results that negate this hypothesis have demonstrated the potential complications of this conclusion, and have high relevance to the seemingly anomalous B-wave augmentation seen in our $50 \mathrm{~V}$ animals. Lei and Perlman used specific $\mathrm{K}^{+}$channel blockers to investigate the ERG B-wave dependence on $\mathrm{K}^{+}$currents, and it was discovered that barium and cesium ions injected intra-vitreally significantly augmented the B-wave, without affecting the A-wave component ${ }^{268}$. Since Müller cells are highly dependent on $\mathrm{K}^{+}$channels for uptake, blocking them should have extinguished the $\mathrm{K}^{+}$current if it were responsible for the B-wave generation, but instead it had the opposite effect. This led the researchers to conclude that the simple Müller cell/ $\mathrm{K}^{+}$hypothesis of B-wave generation was demonstrably incorrect - a subject that is still debated to this day - but also gave 
important insight on what possible mechanisms underlie a selective B-wave augmentation. They concluded that one of three scenarios must be involved: an increase in photoreceptor input to bipolar cells; a direct action on the cells responsible for B-wave generation; or a reduction of cellular components acting to suppress action of cells responsible for B-wave generation. In addition to these, a fourth possibility must also be considered, and that is a potential decrease in resistivity of the tissue through which these electrical currents flow.

An increase in photoreceptor sensitivity. Photoreceptors are unlike many other conducting cells of the body in that their resting membrane potential is slightly depolarized, or more positive, during the absence of stimulus. Where normal neurons have a resting potential of around $-70 \mathrm{mV}$, a photoreceptor rests around $-40 \mathrm{mV}^{269}$. This means that under dark conditions, a persistent electrical current known as the "dark" current is generated by the cell's high $\mathrm{Na}^{+}$permeability. Upon photo-stimulation, the cell becomes hyperpolarized through a complex series of actions that ultimately lead to the closing of $\mathrm{Na}^{+}$channels. This hyperpolarization is what drives the A-wave negative current during light stimulation- the dark current reversal is recorded as a negative spike in the ERG curve ${ }^{270}$. In order for photoreceptors to be more sensitive to light, theoretically this would require the resting membrane potential to decrease, toward that of a normal neuron. Under these circumstances, it would be easier to elicit a response from that photoreceptor cell, rendering it hypersensitive. Practically speaking, this is something that occurs every day, since dark adaptation- or a purposeful increase in photoreceptor sensitivity- allows us to see more clearly in the dark. Without this important mechanism, humans would walk around with night blindness and an inability to see low intensity light levels. Abnormal photoreceptor sensitivity, which can occur under photopic conditions, is a phenomenon that is also observed with phosphodiesterase inhibitors, such as IBMX and theophylline, where ERG data has shown a clear B-wave augmentation and photoreceptor hypersensitivity after administration ${ }^{271}$. The mechanisms behind this effect are not fully understood, but probably lie in the heavy reliance of phototransduction biochemistry on levels of cGMP within the cell. Alternatively, a significant body of knowledge points to calcium as an important feedback molecule in rod photoreceptors, possibly acting through modulation of cGMP levels ${ }^{272}$. Intracellular calcium levels have been shown to affect photoreceptor sensitivity, with moderate levels enhancing phototransduction, and high levels having an opposite effect ${ }^{273}$. The mechanism of calcium-assisted photoreceptor sensitization is more well established, since calcium levels have long been known to play a role in light adaptation $^{274}$.

For discussion in the context of mTBI-associated visual dysfunction, the inflammatory micro-environment that exists within the retina after blast, as evidenced in the histological data, may cause changes in intra- and extra-cellular ion concentrations that yield a similar effect as these pharmacologically-induced cases of photoreceptor sensitivity. From a clinical standpoint, calcium plays an important role in diseases involving long-term inflammation. Often seen clinically, gross, ectopic deposits of calcium can signal the first signs of pathology in a number of human diseases ${ }^{275,276}$. Calcium receptor investigations have shown that it plays an important role as a signaling 
molecule in inflammatory conditions, and its high levels in serous exudate probably act to recruit macrophages to sites of damage ${ }^{277}$. Studies in contusive spinal cord injury have identified abnormally low levels of extracellular calcium 3 hours after injury, owing to cellular uptake of local calcium stores around the site of injury ${ }^{278}$. As discussed in Chapter 1, calcium also plays a role in the primary injury of TBI, with damaged cells often having difficulty with intracellular calcium regulation due to the mechanical insult of the primary injury ${ }^{98}$. It may be the case that alterations of calcium homeostasis after blast initiates a biochemical change in photoreceptors of $50 \mathrm{~V}$ mice that renders them hypersensitive to light, even under photopic conditions. Significantly, our structural studies have found a thinning in the LE photoreceptor layer at 30 days (on OCT), as well as histologically at 10 weeks after blast ${ }^{179}$, indicating proof of photoreceptor damage that may be accompanied by this hypersensitivity. While B-wave augmentation in our experimental animals may not definitively, or even exclusively, be tied to photoreceptor sensitivity, it is important to note that photoallodynia is a commonly encountered symptom of TBI-associated visual dysfunction. Increased critical flicker fusion frequency in individuals with mTBI are directly related to self-reported problems with light and motion sensitivity, hinting that electrophysiological changes in retinal function may give rise to subjective visual hypersensitivity ${ }^{279}$. These facts, paired with the histological evidence of outer retinal thinning, and a B-wave defect in the ERG of these animals, suggest that photoreceptor alterations may play a critical role in the pathology of mTBI-associated vision problems.

Rod-driven on-bipolar cells and loss of lateral inhibition. Photoreceptors seem superficially to be the most important cell in the retina, since they are the major cells involved in photo-transduction. However, the simple on-off dichotomy of photoreceptor activation could never convey the intricate images sensed by the mammalian visual system in all of their rich detail. This requires a complex network of cells acting in concert to organize pertinent visual information, pass this information on to the neurons of the retina, and subsequently the visual centers of the brain. A huge number of interneurons- called horizontal cells, and amacrine cells- provide refinement of the on-off signal from photoreceptors through feedback, feedforward, negative, and positive regulation at synaptic connections to bipolar cells ${ }^{280}$. In passing of information from the photoreceptors to the bipolar cells, horizontal cells in the outer retina and amacrine cells in the inner retina provide lateral feedback to improve the signal before passage to the RGCs. Lateral feedback is largely responsible for temporal and spatial refinement of photoreceptor signals ${ }^{281}$, and may play an important role in conveying directional selectivity to $\mathrm{RGCs}^{282}$.

An important potential cause for the ERG B-wave augmentation in blasted animals is the selective loss of one or many of these regulatory interneurons, specifically those interneurons with negative feedback on bipolar signaling. As discussed previously, the ERG B-wave gives information about activation of cells specifically within the INL, most pertinently to this topic, the bipolar cells. If a negative regulatory subpopulation of horizontal cells were selectively lost due to blast injury, it would cause a loss of negative feedback at the level of the OPL. Functionally, this would yield a decrease in the inhibition of signals passing from the photoreceptors to the bipolar cells, and might 
theoretically be visible as an increase in the B-wave due to increased bipolar cell activity. Loss of negative feedback from amacrine cells in the IPL is more complicated to explain. If an amacrine cell is regulating the synapse between the bipolar cell and the RGC, one might not expect the loss of negative input to have an effect on the B-wave, since bipolar cells would be functioning normally, but their signals to RGCs would be amplified. In reality, amacrine cells synapse with bipolar cells all along the cell body, as well as dendrites and axons ${ }^{283}$, which leaves the possibility for an increase in bipolar cell firing with a loss of inhibitory input at the level of the IPL. Other studies have indicated that a loss of OFF-bipolar cells could cause B-wave increases, since their input seemed to shape and suppress the B-wave under physiological conditions ${ }^{284}$. While it is difficult to understand a mechanism by which only inhibitory interneurons or hyperpolarizing bipolar cells would be lost in TBI, one important piece of evidence does speak to this possibility. As discussed in Chapter 2, retinal histology in this model found a significant decrease in the thickness of the INL, indicating a loss of one or more cell types within this layer. This could include any of the regulatory interneurons, as well as the bipolar cells themselves. So although this seems to be a less likely scenario, it is important to identify it as a possibility nonetheless.

Alterations in tissue resistivity. The electrical circuit recorded in the ERG wave is similar to any non-biological electric circuit in that it can be described by the relationship that voltage is directly proportional to the product of current and resistivity, a relationship commonly known as Ohm's law ${ }^{285}$. If one assumes that no cellular changes in function have caused the ERG B-wave augmentation, a final possibility still exists for why this seemingly paradoxical finding occurs. That is, the voltage change is not caused by a change in the current, but instead by a decrease in the resistivity of the retinal circuit through which the current flows. In biological terms, tissue resistivity is dependent upon a number of factors, including water, ion, air (in the case of the lungs), and mineral content ${ }^{286}$. Of the body tissues, blood has one of the lowest resistances, at $100 \Omega \mathrm{cm}$, while normal cell-dense tissues may be closer to $1000 \Omega \mathrm{cm}^{287}$. With reference to ocular resistivity and its effect on ERG readings, this has been long recognized as a potential complicating factor in ERG result interpretation, especially when recording from the cornea $^{288}$. With corneal-surface ERG readings, electrical current flows through a number of higher resistivity areas in the outer retina, toward the vitreous humor, the socalled low-resistivity shunt, before circling back toward the originating cells in the retina to complete the circuit ${ }^{215,289}$. An important possibility to consider with the ERG findings in our experiments is the potential decrease in resistance of the retina as a result of the inflammatory microenvironment.

Examples of physiologically-induced changes in tissue resistivity are surprisingly well documented in the literature. Both temperature and hematocrit have been shown to alter resistivity of human blood ${ }^{290}$, while necrosis and edema produced significant decreases in muscle tissue resistivity after traumatic electrical burns ${ }^{291}$. Fluid movement in the form of edematous extracellular fluid accumulation is a common finding in both brain and retinal trauma ${ }^{176,292}$, and its high electrolyte and protein content explain why it would cause a decrease in the resistivity of any affected tissue ${ }^{293}$. There is no overt evidence of edema in the retinas of our $50 \mathrm{~V}$ animals after blast, but previous experiments 
have found about a $0.08 \%$ increase in the fluid content of the brain at 1 day after blast ${ }^{179}$. Edematous expansion could account for the lack of total retinal and INL thinning in the LE of $50 \mathrm{~V}$ animals at 30 days after blast on OCT, even though histology of these same eyes at 3 months shows signs of significant retinal thinning. The OCT in vitro method would preserve this artificial water content and make the retina appear to be thicker even in the presence of significant cellular losses. One important consideration to weighing the possibility of this explaining the ERG findings is that a decrease in the resistivity of the retinal tissue should theoretically yield an increase in the peak amplitude of the entire current across the ERG function. This means one would expect the A-wave to have an increase in peak amplitude as well, since the decreased resistance would make both the negative and positive voltage travel with greater ease through the circuit. Although it was far from significant, the ERG in the left and right eyes did show a trend toward having a higher peak amplitude than the control eyes. It is difficult to say whether this trend should be considered as part of this story, but it certainly raises the probability of tissue resistivity being at least partially responsible for the ERG findings in blasted mice. Given the possibilities discussed, it seems most reasonable to conclude that the ERG changes are probably due to more than one factor, but nonetheless indicate a significant functional change in the eyes of blasted animals, that is improved by treatment with SMM-189.

\section{Optokinetics}

Optokinetic nystagmus $(\mathrm{OKN})$ refers to the eye movement involved in reflexively tracking a large scale displacement of the visual field. It serves to stabilize the visual field on the retina when the visual field is being moved ${ }^{294}$. OKN is a visual function that can easily be elicited in both humans and animals by horizontally moving vertical stripes that cover a large part of the visual field. The OKN reflex is mediated by a collection of visual nuclei and circuits in the brain known collectively as the accessory optic system (AOS) ${ }^{295}$. The optokinetic (OKT) testing device used in our experiments allows free movement of the experimental subject, and thus OKN is gauged by the observation of an animal tracking (head movement) the moving pattern. Tracking behavior, usually called optomotor tracking, is generally accepted to be a good indicator of the OKN reflex, and has been reliably used to assess visual function in mice ${ }^{183}$. Using the OKT device, vertical stimulus bars can be changed to test a subject's visual acuity and contrast sensitivity thresholds, since the OKN reflex is only present when a stimulus is at or above the perceptible level for that subject.

The OKT response is known to be mediated almost exclusively by sub-cortical visual areas in the brain, and is not altered even with full cortical ablation ${ }^{183}$. While the neurobiological underpinning of the OKN reflex is relatively well understood, the pathway underlying the initiation and execution of the optomotor reflex observed in the OKT response is much less so ${ }^{183}$. Optokinetic nystagmus, visual orienting, and motor movements involved in the execution of tracking must ultimately rely on an intact retinofugal system ${ }^{295}$, including the tectospinal (colliculospinal) tract ${ }^{65}$ and accessory optic system ${ }^{295}$. Cranial nerves driving extraocular muscles as well as cervical motor 
circuits, must be intact to coordinate head, neck and eye movements. Finally, the perception of spatial or contrast information is, of course, reliant upon the intact function of photoreceptors, ganglion cells, and directionally-selective cells within the retina ${ }^{296}$. Put together, these features allow OKT to serve as a sensitive and comprehensive test of the visual system. However, this heavy reliance on the function of so many disparate pathways can be a double-edged sword. With the involvement of the entire sub-cortical visual pathway, it is difficult to localize lesioned areas responsible for a complex pathology such as that seen in our model. The OKT results in these experiments highlight this weakness clearly, since some of the results seem contradictory, and have conflict with our own previously published data. To fully understand and scrutinize the VA and CS data found in these and previous experiments, it is first necessary to briefly discuss the complicated pathway involved in eliciting the OKT response.

The pathways involved in the $\mathrm{OKN}$ and optomotor response. The axons leaving the eye and projecting to brain regions involved in visual processing compose the so-called retinofugal pathway. These fibers contain all sensory information from the retina projecting to systems involved in both OKT and cortical vision. With relevance to the OKN response, stimuli moving rapidly across the visual field will initiate signals sent from RGCs in the retina to the pretectal nucleus of the optic tract (NOT) and other terminal nuclei of the AOS also located in the midbrain ${ }^{294}$. The NOT, as well as the dorsal terminal nucleus (DTI), contains directionally selective neurons that are known to play a critical role in the initiation of OKN to horizontally moving stimuli ${ }^{294}$. Retinal ganglion cell axons leaving the retina also project directly to the superior colliculus ${ }^{65}$. The superior colliculus (SC) is a layered structure divided into superficial and deep layers, whose cells serve differential functions in relaying visual signals received from RGCs in the retina. The cells in the upper layers of the superior colliculus project to the thalamus for relay to visual association areas that assist in perception and coordinating eye movements ${ }^{297}$, while the deep layers of the superior colliculus project through the contralateral tectospinal tract to coordinate head movements for visual orienting ${ }^{298}$. Previously in our TBI model, the superior colliculus was found to contain a population of highly reactive microglia, indicating a possible role for SC-coordinated dysfunction in yielding functional vision defects. Coordination of eye and head movements are integral for normal visual function, and dysfunction in this coordination is a commonly encountered sign in human cases of mTBI-associated visual dysfunction ${ }^{53}$. Since axons throughout the left optic nerve are lost, it is possible that signal loss to any of these important visual nuclei could yield OKN defects observed in this model.

Visual acuity. Visual acuity testing in animals 30 days after blast found no difference between any group, in either eye, in the present study. This is in contrast to previously published data in this model, where both the left and right eyes of blasted animals had significant deficits at 3 weeks post blast, with the right eye deficit being more severe ${ }^{181}$. The reason for this disparity most likely stems from the different experimenter administering the tests in these studies versus that of the previous studies. The optokinetic test can be confounded by human variation, since it is a subjective judgment by the observer to determine if the mouse has moved its head or not in response to a given stimulus. Thus, if a viewer only detects robust head movements as tracking 
movements, and if a treatment makes movements less robust, that viewer might detect no movement, while another viewer might detect movement. The former viewer would then report a deficit and the latter not. As the viewer is always blinded to treatment and stimulus, detection of a deficit in a treated group is objective and unbiased. Nonetheless, a different observer might not detect the same change due to their greater ability to detect an OKT tracking movement. Thus, the prior data indicate that TBI does yield an OKT defect on the VA task, but paradoxically that defect will be more difficult to detect by an observer who more readily detects OKT tracking. Consistent with this, the CS defect detected here is less than in our prior study at around the same time point.

Notably, 4x multi-blasted animals tested by this same experimenter showed significant defects in visual acuity at a year post-blast. In these animals, the injury and defect are likely much more severe, and accordingly were evident even to a more sensitive observer. At this point in time, there still appears to be some debate on whether concussive and sub-concussive events can accumulate over time to yield long-term deficits in the brain ${ }^{157}$. This is despite the high profile finding of CTE in football players and other athletes, even those with no history of concussive injury ${ }^{21}$. Experimental verification that long-term injuries can result from multiple sub-concussive events holds great importance in the field of mTBI research, specifically as it relates to high-impact sports and military applications.

Contrast sensitivity. Contrast sensitivity is the ability to distinguish between different intensities of light and dark. This is what conveys the crispness to vision, and is important in night vision and other instances where gradients between light and dark are minimal. Contrast and gain detection seem to be an intrinsic property of cells both within the retina and in the retinofugal projection nuclei, the LGN and SC ${ }^{299}$. Additionally, the primary visual cortex may also play a role in contrast detection in more challenging situations that require integration of higher order processes for perception ${ }^{300}$. In $50 \mathrm{~V}$ animals, contrast sensitivity thresholds measured at 6 different spatial frequencies 30 days after blast demonstrated a severe deficit when compared to control animals. Both eyes were affected, with the right eye showing a more substantial deficit than the left. Drug treatment remedied this deficit, bringing the CS functions of 50SMM animals back toward control levels in both eyes. Since the LE at this time point shows thinning of the photoreceptor layer and a loss of RGC axons running through the optic nerve and tract, it is logical to conclude that these must be at least partially responsible for the functional CS deficits in the LE of these animals. Additional evidence for this hypothesis is that drug treated animals show significant improvements on both of these histopathological readouts, indicating they may be linked to the functional improvements seen with drug treatment as well.

Oddly, the right eye at this time displays no such histopathology, and as was seen in previous VA testing at 3 weeks from blast, displays a paradoxically more severe deficit than the left eye. This gives strong indication that the right eye deficit is not only different in pathophysiological mechanism that that of the left eye, but may actually be linked to damage in more distal visual processing nuclei, which correspond to the left side of the brain. The axon projections from the right eye, in both the optic nerve and 
tract, show very few signs of damage compared to the left eye, at both acute and extended time points, and by a number of different markers of pathology. This speaks to the potential for some, if not all, of the visual acuity deficit in the RE to be traced back to more central visual system causes. Damage to the retinofugal pathway could be mediated by loss of connectivity in visual nuclei projections, or even loss of second-order neurons within those nuclei. Recent investigations in the mouse lateral geniculate nucleus have identified cells with high specificity for single-directional movement ${ }^{301}$, meaning their loss on the left side of the brain due to blast might mediate a significant defect in the RE visual response. Visual deficits in this eye could also be due to a defect in the initiation of saccadic response, or coordinated head movements, due to loss of cells in the superior colliculus who are integral to these functions. An experiment in cats validated that unilateral ablation of the superior colliculus causes a loss of the OKN reflex in the eye contralateral to the lesion ${ }^{302}$. Denervation of the superior colliculus from RGC axon degeneration could result in eventual SC neuronal degeneration, a phenomenon that has been demonstrated experimentally in rats ${ }^{303}$, or direct effect of the blast injury might injure SC neurons on the left side of the brain, giving rise to at least part of the RE visual deficit.

Multi-blast animals displayed significant deficits in CS at a year out from blast, but interestingly had an apparent reversal of which eye was more severely impacted. In these animals, 50-psi yielded more significant deficits in the LE than the RE. However, if one compares the CS threshold at only $0.272 \mathrm{c} / \mathrm{d}$ in $50 \mathrm{~V}$ animals at 30 days post-blast, it is clear that the LE threshold is actually increased compared with the RE at this spatial frequency. It may be the case that the entire CS function in long-term multi-blast animals more closely resembles the function of $50 \mathrm{~V}$ animals, but further studies will be needed to determine this. Also of note, where the LE threshold of $50 \mathrm{~V}$ animals 30 days post-blast is around $48 \%$ for detecting $0.272 \mathrm{c} / \mathrm{d}$, the long-term multi-blast animals required nearly $60 \%$ to see this spatial frequency. Interestingly, when comparing the RE of 30 day $50 \mathrm{~V}$ animals to 50-psi multi-blast animals a year out, the CS threshold is almost unchanged, speaking yet again to the different mechanism that must exist between the two eyes. Since the multi-blast animals would presumably have a more severe injury than singleblast animals, it is difficult to make direct comparisons between the two. Specifically, it is impossible to conclude whether the more severe LE deficit in multi-blast animals comes from long-term injury, multiple blasts, or both.

Future research strategies. Contrast sensitivity and visual acuity are highly sensitive, but non-specific tests in identifying retinal and visual system disease ${ }^{182}$. It is evident that by these two metrics, mice have subtle, but evident, visual system changes that would contribute to significant difficulties in the activity of daily life. Further studies should be done to investigate changes in both pre-blast and post-blast VA/CS measurements at many more time points after blast, including a critical period from the first weeks to the first months after blast. The data in this dissertation conflicts with that of previous studies in this model, and should be confirmed over multiple time points to clarify the true nature of the functional visual defects. OKN should optimally be combined with corresponding ERG/pERG, and OCT measurements. Additionally, stereological counts of the LGN, SC, and visual cortices should be undertaken, to 
determine whether a diffuse loss of cells from one or more visual brain regions may contribute to the complex pathology of mTBI-associated visual dysfunction in this model.

\section{A Discussion of Injury Mechanisms and Inconsistencies}

\section{Axonal shearing and stretching due to head, eye, and brain movement}

One purpose of these experiments was to further investigate the mechanism of injury in mTBI-associated visual dysfunction. The histological and functional data have indicated that at least one part of the injury must undoubtedly come from the axonal shearing, but the secondary question then becomes when and where does this shearing occur, and what makes the visual system so particularly vulnerable to this? In previous experiments, animals at one day after blast showed no changes in the optic tract. Many studies have shown that, in axons directly harmed by primary injury, axon bulbs often become visible within hours, and can take as long as 24 hours ${ }^{251}$. The fact that the OT seems relatively normal at this time point indicates that points of axonal breakage are probably located more proximal to the eye. As discussed previously, at 3 days after blast, there was found to be a localization of bulbs in the left $\mathrm{ON}$ that were much larger and more numerous than those in the OT at the same time, indicating that a significant point of stress may be in the bony canal of the skull that serves as the passageway for the ON to leave the orbit of the eye and enter the brain case. The eye and brain have a significant amount of freedom to move within their bony enclosures ${ }^{304}$, with the eye having an additional opening through which more extensive movement is clearly possible. Complex biomechanical analysis using computer simulations have demonstrated that shock waves incident upon the head may transmit energy in the form of compression, tension, and shear stress waves ${ }^{305}$. Together with the measured head movement, these forces may cause the brain and eyes to move in opposing directions during the milliseconds after shock wave incident. Since the ON is held fixed by its bony canal, this may act as an axis of torque, about which mechanical breakage of axons would inevitably occur. Shock waves can also act directly on the skull, causing a very small but measurable compression, which can then act to raise intracranial pressure ${ }^{306}$. Interestingly, increases in intracranial pressure are now being identified as potential causes for biomechanical stress injury at the optic nerve head ${ }^{245}$, another area where our previous studies have found axon bulbs at 3 days after blast ${ }^{179}$.

If a focal injury of the proximal visual tracts lies at the heart of the primary injury in our model, downstream axonal damage (in the OT and distal) may be axons undergoing Wallerian degeneration due to upstream breakages. Unlike in humans and some other mammals, the RGC axons of mice do not maintain a retinotopic organization once they leave the retina, and instead exhibit a highly random arrangement in the optic chiasm and optic tract ${ }^{307}$. This may explain the seemingly random arrangement of damaged axon fibers, uniformly spread throughout the OT and in no identifiable pattern. However, it is important to note that SMM-189 treatment did significantly decrease the number of axon bulbs in the OT at 3 days. Since drug treatment, and immune 
modulation, may not be able to salvage the axons damaged in the primary injury, it may be the case that a majority of the damaged axons seen in $50 \mathrm{~V}$ right OT at 3 days are due to some secondary injury. The absence of significant axon rescue in SMM-189 treated left ONs at 11 weeks hints that perhaps some initial wave of axonal damage is occurring more proximal to the eye at time points prior to 3 days, and that the axons seen in the OT at 3 days and beyond are due to secondary inflammatory damage. However, there is at least some evidence that even axons damaged by primary injury may have a critical period during which they might be saved, if acted upon by the correct regenerative mechanisms ${ }^{308}$. SMM-189 may polarize microglia in such a way that they release trophic factors needed for this regenerative pathway to dominate in many axons. A schematic overview of this hypothesis can be seen in Figure 6-7.

\section{The problem of the left eye-right eye difference}

A persistent difference between the left and right eye permeates the structural and functional vision end points, hinting at a different injury mechanism at work in each eye. Potential injury mechanisms of the left eye were discussed in the previous section, with a hypothesized stress locus at the $\mathrm{ON}$ at the point of the bony canal and the ON head, with subsequent secondary injury. Functionally, the right eye shows both short-term (in these experiments), and longer term (previous studies) deficits in VA and CS by measure of the OKN reflex. As recounted earlier in that section, these RE deficits can almost entirely be explained by direct injury to the visual nuclei on the left side of the brain (blast side). With that being said, there still exist some histological evidence of RE injury that must be relevant in any explanation for the RE deficit, and vice versa. In previous experiments, the right retina was found to be significantly thickened at 10 weeks from blast, and in our current studies, the RE had a significant microglial presence at 30 days after blast. Initially, this seemed like there may be direct injury to the RE, potentially from the eye being compressed into the foam during blast administration. However, at this same time point, GFAP reactivity was not seen, where it was seen in the LE. Furthermore, in the left OT, very few damaged axons were seen at early time points after blast, and the small number present can reasonably be attributed to the $1-10 \%$ percent of axons whose fibers remain uncrossed from the left eye. These contradictory findings leave a puzzle as to why there is any damage to the RE at all, and why this pathology seems to differ from that of the LE so significantly.

An important possibility to consider for the findings in the right eye is that the inflammatory response from the LE may give rise to inflammation in the RE, even in the absence of any direct damage. This inflammatory response might then cause direct damage to the retina or RGC axons of the RE, leading to functional deficits in that eye and long-term axon loss in the optic nerve. Significant precedent exists for this as a mechanism in many diseases affecting the visual system ${ }^{309}$. It is a well-known phenomenon that infections can spread from one eye to another, a condition termed Sympathetic ophthalmia, sometimes leading to disastrous results in the contralateral eye

310. Indeed, this may have been the reason for Louis Braille's complete vision loss as a child ${ }^{311}$. Studies of direct ocular globe penetration reveal significant monocyte and 
lymphocyte invasion into the non-injured eye ${ }^{312}$. Relevantly, direct injury to the RGC axons in the $\mathrm{ON}$ has also been shown to cause microglial reactivity in the contralateral uninjured eye ${ }^{313-315}$. One paper postulated that the injury signals from uncrossed fibers in the damaged optic nerve may be contributing to damage in the nuclei of the unaffected eye by releasing local inflammatory mediators ${ }^{315}$. Interestingly, two studies found the contralateral eye to have evident GFAP reactivity, albeit less than in the ipsilateral eye, that was found predominantly in astrocytes in the NFL ${ }^{314,316}$. This is similar to what was found in our animals at 30 days after blast, since the right eye qualitatively seemed to have more GFAP in the NFL, but did not have the large number of processes penetrating into the IPL as the LE.

\section{SMM-189 Benefit and Implications Going Forward}

These experiments validated the use of SMM-189 in a mouse model of mTBI, with many functional and histologic measures of benefit over vehicle-treated animals. SMM-189 was hypothesized to act through alterations in microglial polarization, potentially shifting the polarization state from an M1 to an M2 phenotype. Our experiments have partially confirmed this hypothesis, since many readouts of immune system activation are altered in drug-treated animals, and these alterations are accompanied by functional and structural benefit on disease pathology. Furthermore, M2-like microglia were increased significantly in damaged OTs of animals receiving drug. However, these experiments have also highlighted the complex nature of in vivo microglial polarization, posing more questions about precisely how the drug is affecting microglia in the multifaceted environment created after trauma.

\section{The complicated issue of microglial polarization}

Traditionally, macrophages have been forcibly categorized into two dichotomous states, being either classically or alternatively activated. New research on macrophages has amended this belief to recognize the variety of activation states, including extensive overlap, according to the actions and central role of the macrophage in specific states of disease or physiologic condition ${ }^{258}$. It is documented that microglia can take on mixed M1/M2 phenotypes due to conflicting pro and anti-inflammatory cytokine release, and for this reason it becomes important to observe the balance of phenotypic markers relative to one another, rather than in isolation ${ }^{317}$. According to the literature, at least 4 distinct macrophage polarization phenotypes have been traditionally lumped into the M2 "alternative" activation group: M2a, M2b, M2c, and Mox ${ }^{116,318}$. M2a microglia are more close to the typical understanding of M2 polarization in that they are primarily antiinflammatory in effect, promote tissue repair, and release anti-inflammatory cytokines IL-1 and IL-10, while M2c (often found with M2a) are more consistent with the resting immunosuppressive "M0" state ${ }^{319}$. The M2b activation state is a mixed-phenotype, involved in regulatory $\mathrm{T}$ cell recruitment and immune modulation ${ }^{320}$. Despite the extensive literature identifying these M2 class subtypes, little research has been done on the role of M2b microglia in specific disease pathologies, and how they may help to alter 
the immune response in a favorable way. It is probably most beneficial to view their actions as primarily regulatory, meaning their presence can be positive or negative, depending on the pathology and its return to homeostasis.

In vitro cytokine assays on human microglia have demonstrated SMM-189's ability to alter a variety of pro and anti-inflammatory cytokines and chemokines released after LPS stimulation ${ }^{181}$. The expression levels of LPS-stimulated microglia after SMM189 treatment mimics almost identically those seen in type M2b microglia in a recent study investigating different microglial activation states ${ }^{321}$. In M2b microglia, as opposed to M2a and M2c types, levels of TNF-alpha, IL-6, IL-10, and IL-12 are still significantly elevated, although less so than M1 microglia. This speaks to the somewhat mixed picture of M2b microglia, since they are still actively involved in recruiting peripheral T cells and acting as APCs ${ }^{322}$. In our IHC studies there was a significant level of CD206 in individual microglia of the ROT in all groups, and interestingly, M2b microglia are not known to express CD206. Taken together, these indicate there should be a mixed M2 phenotype being expressed by drug-treated animals, with a selective polarization to an M2a/M2b phenotype. Given the immensely complex nature of microglial polarization, it is probably not possible to determine a microglial phenotype based on a single cell surface receptor. Investigating the specific M1 and M2 categorization of microglia in drug-treated animals should be planned for the future, giving a more complex understanding of not only what, but why these different microglial phenotypes might prove more or less beneficial in mTBI pathology.

\section{Cannabinoids as immune modulators: Issues, benefits, complicating factors}

CB2 receptor tissue distribution. Many studies have demonstrated the use of cannabinoids acting on both $\mathrm{CB} 1$ and $\mathrm{CB} 2$ receptors in regulating the immune response 198,323 . What continues to be problematic is identifying the ways and locations in which these molecules exert their effects. Aside from their known presence on immune cells, including microglia, the cellular and tissue distribution of $\mathrm{CB} 2$ receptors on non-immune cells continues to be a hotly debated topic ${ }^{324,325}$. While much research has indicated an absence of $\mathrm{CB} 2$ receptors on non-immune cells in the CNS, an equally significant body of evidence demonstrates their presence on many different neural and non-neural cells (see Atwood and Mackie 2010 for an excellent review ${ }^{325}$ ). Studies have identified CB2 receptors on neurons within the cerebellum and brain stem ${ }^{326}$, in the hippocampus, striatum, cerebral cortex ${ }^{327}$, and even in the retina ${ }^{328}$. One study postulated the existence of CB2 receptors on neurons in virtually every major area throughout the brain, strengthening their IHC evidence with neuropsychobiological correlates corroborating this tissue distribution ${ }^{329}$. Recent studies have hypothesized a potential role for endocannabinoids in regulating cerebral blood flow, with particular importance on CB2 receptor activation ${ }^{330}$. A study in humans found $\mathrm{CB} 2$ receptors were present on vascular endothelium, but not nearby astroglia, indicating their potential role in blood flow regulation ${ }^{331}$. Other studies have confirmed this finding ${ }^{332}$, and as a result cannabinoids are now being investigated for their use in ischemia/reperfusion injuries in the heart and liver ${ }^{330,333}$. 
The studies undertaken in this dissertation show that SMM-189 acts on microglial cells in the brain, altering polarization in at least a subset of cells near sites of known injury. It is also clear that the drug decreases a number of pathologic findings present after mTBI in our model. The possibility of some additional benefit occurring due to SMM-189's action at other cellular locations cannot be ruled out. For example, if SMM189 acted even partially at the neurovascular endothelium, to heal a leaky BBB, this could convey a significant benefit to the long term outcome in mTBI. Direct action at neurons in the brain, or RGCs in the retina, could also temper programmed cell death pathways to yield the long-term benefits seen in our model. Determining if CB2 receptors are actually present centrally on non-immune cells, and whether some of the beneficial effects of cannabinoids in a number of disease processes are indeed due to nonimmune cell effects, remain critical questions in understanding and using cannabinoid pharmacotherapy going forward, and may provide additional mechanisms of action for how SMM-189 is exerting its beneficial effects in our model.

Cannabinoids and their receptors: Beyond CB1 and CB2. There is a significant body of evidence that indicates the presence of additional receptors to which cannabinoids may act as ligands ${ }^{186}$. One heavily researched non-psychotropic phytocannabinoid, cannabidiol (CBD), has been proposed to exert its action through a variety of receptors and modulatory targets, including glycine, opioid, TRPV, and 5-HT $1 \mathrm{~A}$ receptors, amongst others ${ }^{334}$. In addition, new evidence also poses the possibility that endogenous molecules released during times of inflammation may act to allosterically regulate $\mathrm{CB}$ receptor function, further modifying action of both endogenous and exogenously administered cannabinoids in vivo ${ }^{335}$. Many of the anti-inflammatory phyto-cannabinoid activities are now known to be mediated through non-CB1/CB2 receptor activity ${ }^{336}$, raising the possibility that the drug used in our studies, SMM-189, may be acting via an additional receptor to convey its healing effects in mTBI. Extensive pharmacology studies on SMM-189 in CBR-transfected human embryonic kidney cells have shown intracellular cAMP levels to be definitively linked to the drug's action at CB2 receptors ${ }^{218}$. These studies have also confirmed that SMM-189 exerts no functional activity at CB1 receptors ${ }^{218,337}$. Assays used in previous studies of SMM-189 could only account for $\mathrm{CB} 1$ - or $\mathrm{CB} 2$-mediated changes in cAMP, so there is a possibility that the drug may act on other receptors giving rise to off-target effects. Future studies should be aimed at investigating this to more thoroughly understand both the mechanism of the drug's action, and potential off-target effects.

Natural and synthetic cannabinoids present an exciting future in pharmacotherapeutics, with their ability to potently and selectively act on the body's immune system to suppress undesirable activation. It is, however, important to recognize that immune system modulation is not a desirable target in all diseases, and in the case of cancer can even prove devastating ${ }^{338}$. As the notorious psychedelic scholar and selfproclaimed "altered-statesman" Terence McKenna once said to a doctor, when remarking on the potential for phyto-cannabinoids to cure cancer ${ }^{334}$ after his own devastating diagnosis of glioblastoma multiforme (GBM), "Listen, if cannabis shrinks tumors, we would not be having this conversation," 339 . While cannabis and phyto-cannabinoids alike, will probably not represent the magic key to curing all immune-mediated diseases, 
their potential to offer molecules for therapeutic investigation - particularly within the realm of neurotherapeutics - is undeniable and important to recognize. Indeed, this dissertation has demonstrated the vast improvements a purposefully-designed cannabinoid can convey in an immune-mediated pathology with few currently available treatment options. The complex pharmacology and receptor interactions of cannabinoids, paired with their proven efficacy in providing novel therapeutics, make experimental investigations of their mechanisms and myriad of effects an important and worthwhile pursuit. It is our hope that these studies can help de-stigmatize the idea of cannabinoidbased therapeutics, and pave the way for future studies into these fascinating molecules.

\section{Study Limitations}

As with all research, certain limitations existed within our studies. Male mice were used exclusively within our experiments, since this model was previously characterized using only male mice. Because of this, it is unknown what effect both blast exposure and SMM-189 treatment would have on female mice. Furthermore, as with all animal models it is not known how SMM-189 would improve outcomes in human cases of TBI. With regard to the drug SMM-189, in vivo dose-response studies were not done to determine if the dose we used in these experiments was the optimal dose, so it may be the case that this dose was above or below the ideal therapeutic level. As discussed previously, SMM-189's mechanism of action is not fully understood, which presents a limitation in interpreting the improvement after drug administration. Another limitation that has been touched on previously exists in interpreting the M1 and M2 polarization information from the ROT. Having used only two immunomarkers to distinguish activation states, we were not able to fully identify microglia as being M1 or M2 polarized, and instead could only say definitively which of the two markers was expressed to a greater extent in a specific cell. Finally, the acute time points had sample sizes that were very small, significantly limiting our ability to achieve statistical significance, and to detect subtle changes. Other limitations have been previously identified and discussed throughout the text, some of which will be addressed in the proposal of future studies.

\section{Future Research Strategies}

Going forward, many avenues for investigation remain of great interest, and there are many possible experiments that could shed light on some of the more complex discoveries of this dissertation work. Longitudinal behavioral and histologic studies on single-blasted animals treated with SMM-189 should be undertaken to determine longterm benefits in this model. These investigations should include OKN and ERG testing,

as well as OCT. Ideally, tests should be administered prior to blast, and then at acute and long-term time points after blast, in order to track changes over time within the same animal. Many changes seemed to occur in this model between 30 days and $2+$ months after blast. For this reason, it would be particularly important to gather data consistently over the first few weeks and months after blast, and then decrease the testing interval 
after 2 months for longitudinal studies. Another functional test that may give insight into the mTBI blast injury is pattern ERG (pERG), an electrophysiological readout highly sensitive for RGC function ${ }^{340}$. Pattern ERG changes have been seen prior to the onset of visual function changes, and may serve as an early indicator of RGC dysfunction or loss in these animals. Since a common finding in human cases of mTBI-visual dysfunction is extrocular muscle defects, testing of extraocular muscle function using high speed videooculography ${ }^{341}$ would be an important functional test to add to the repertoire of visual system description in this model.

Future studies should ideally seek to clarify the complex effect of blast injury on the extra-retinal visual system. In addition to obvious damage to the retina and optic nerve, many of the signs and symptoms seen in mTBI-associated visual dysfunction can be reasonably traced back to disruption of retinofugal projection regions. To visualize retinal projections, and investigate their potential loss after blast, intraocular injection of Cholera toxin B conjugated to fluorescent dye should be used in combination with IHC to image both excitatory and inhibitory input to the SC and LGN, or other visual accessory nuclei ${ }^{342,343}$. Some of the data in our experiments hinted that at least part of the RE function deficits may in fact be linked to loss of left brain visual nuclei inputs or neurons. If synapses are decreased in any or all of these areas, it would yield a potential pathophysiological correlate for this disease and associated visual dysfunction. Additionally, stereological counts of NeuN+ cells in the specific visual nuclei would aid in achieving this end.

Importantly, further investigation of the SMM-189 drug benefit in this model should include detailed IHC studies in animals from 24 hours to 7 days after blast, immunostaining for a number of cellular markers associated with the diverse microglial phenotypes ${ }^{258}$. Our data found right OTs of blasted and drug-treated animals to have a microglial population expressing both M1 and M2-associated markers, but at differing ratios. Since the literature touches very little on these mixed-phenotype microglia, and they seem to play a critical role in positively modulating the immune response to trauma, it is important to investigate phenotypic profiles of microglia in blasted animals both treated and non-treated with SMM-189. More complex studies might include rtPCR of cytokine and chemokine expression from highly damaged areas, such as the left $O N$, in order to more completely describe the microglial polarization states in blasted animals. Other histologic investigations should aim at microglial polarization in the retina and optic nerve, during the first week after blast, to characterize the microglia in these regions as well. Also in the optic nerve, more thorough axon counts should be done using EM, as opposed to LM, since many of the smallest axons can not be visualized on LM alone. At least some evidence suggests that axon caliber may be tied to RGC type, and since a particular type of RGC may be selectively lost in mTBI, it is an important aspect to consider in the future ${ }^{344}$. 


\section{Closing Remarks}

It is clear from the experiments in this dissertation that this mouse model of overpressure blast-induced mTBI with associated visual dysfunction shows a complex and multifaceted disease process. Histopathology has provided significant insight into the underlying causes of visual function deficits, and speaks to the substantial role of neural immune cells in responding acutely to trauma in the eye and visual pathways of the brain. SMM-189 has continued to show promise as a neurotherapeutic after mTBI, significantly alleviating many of the functional and histopathologic findings after blast administration. Its role in altering the immune system is clear, and is likely more complex in vivo than previously hypothesized. Future research should be aimed at investigating the extra-retinal causes of mTBI-associated visual dysfunction, with continued emphasis on functional-structural correlates in this model, as well as determining more comprehensively how SMM-189 alters microglia in a traumatically inflamed state. Our hope is that these studies may add to the significant body of knowledge on a mild disease that is exceedingly common, and often life-altering, providing hope that neuroinflammatory modulation may provide the key to efficacious treatment in the future. 


\section{LIST OF REFERENCES}

1. Hyder, A. A., Wunderlich, C. A., Puvanachandra, P., Gururaj, G. \& Kobusingye, O. C. The impact of traumatic brain injuries: a global perspective.

NeuroRehabilitation 22, 341-353 (2007).

2. Laskowitz, D., Grant, G., Cole, W. R. \& Bailie, J. M. Neurocognitive and Psychiatric Symptoms following Mild Traumatic Brain Injury. (CRC Press/Taylor and Francis Group, 2016).

3. Langlois, J. A., Rutland-Brown, W. \& Thomas, K. E. Traumatic brain injury in the United States: emergency department visits, hospitalizations, and deaths. (2004).

4. Buck, P. W. Mild traumatic brain injury: a silent epidemic in our practices. Health Soc Work (2011).

5. Feldman, R. P. \& Goodrich, J. T. The Edwin Smith Surgical Papyrus. Child's Nerv Syst 15, 281-284 (1999).

6. Kamp, M. A., Tahsim-Oglou, Y., Steiger, H.-J. \& Hänggi, D. Traumatic brain injuries in the ancient Egypt: insights from the Edwin Smith Papyrus. J Neurol Surg A Cent Eur Neurosurg 73, 230-237 (2012).

7. Giza, C. C. \& DiFiori, J. P. Pathophysiology of Sports-Related Concussion An Update on Basic Science and Translational Research. Sports Health: A Multidisciplinary Approach 3, 46-51 (2011).

8. Laker, S. R. Epidemiology of Concussion and Mild Traumatic Brain Injury. PMRJ 3, S354-S358 (2011).

9. Cassidy, J. D., Carroll, L., Peloso, P. \& Borg, J. Incidence, risk factors and prevention of mild traumatic brain injury: results of the WHO Collaborating Centre Task Force on Mild Traumatic Brain Injury. J Neuroinflammation (2004).

10. Laskowitz, D., Grant, G., Leo, P. \& McCrea, M. Epidemiology. Current Opinion in Pharmacology 26, 1-12 (CRC Press/Taylor and Francis Group, 2016).

11. Centers for Disease Control and Prevention (CDC). Report to Congress on Mild Traumatic Brain Injury in the United States:. 1-56 (Centers for Disease Control and Prevention, 2003). at $<\mathrm{http}: / /$ www.cdc.gov/traumaticbraininjury/pdf/mtbireport-a.pdf $>$

12. Defense, D. O. \& Affairs, D. O. V. Management of Concussion/mild Traumatic Brain Injury. 1-112 (2009).

13. O'Neil, M., Carlson, K. \& Storzbach, D. Complications of Mild Traumatic Brain Injury in Veterans and Military Personnel: A Systematic Review. Vol Project\# 05-225. (2012).

14. Feigin, V. L. et al. Incidence of traumatic brain injury in New Zealand: a population-based study. The Lancet Neurology 12, 53-64 (2013).

15. Bazarian, J. J. et al. Mild traumatic brain injury in the United States, 1998-2000. Brain Inj 19, 85-91 (2005).

16. Centers for Disease Control and Prevention (CDC). Traumatic Brain Injury in the United States: Epidemiology and Rehabilitation. (2015). 
17. Langlois, J. A., Rutland-Brown, W. \& Wald, M. M. The Epidemiology and Impact of Traumatic Brain Injury: A Brief Overview. $J$ Head Trauma Rehabil 21, 375 (2006).

18. Baugh, C. M., Kroshus, E., Daneshvar, D. H. \& Stern, R. A. Perceived Coach Support and Concussion Symptom-Reporting: Differences between Freshmen and Non-Freshmen College Football Players. The Journal of Law, Medicine \& Ethics 42, 314-322 (2014).

19. Guilmette, T. J. \& Paglia, M. F. The public's misconception about traumatic brain injury: a follow up survey. Arch Clin Neuropsychol 19, 183-189 (2004).

20. Omalu, B. I., Bailes, J., Hammers, J. L. \& Fitzsimmons, R. P. Chronic traumatic encephalopathy, suicides and parasuicides in professional American athletes: the role of the forensic pathologist. The American Journal of Forensic Medicine and Pathology 31, 130-132 (2010).

21. McKee, A. C., Cantu, R. C., Nowinski, C. J. \& al, E. Chronic traumatic encephalopathy in athletes: progressive tauopathy following repetitive head injury. J Neuroinflammation (2009).

22. Blennow, K., Hardy, J. \& Zetterberg, H. The Neuropathology and Neurobiology of Traumatic Brain Injury. Neuron 76, 886-899 (2012).

23. Jones, E., Fear, N. T. \& Wessely, S. Shell shock and mild traumatic brain injury: a historical review. Am J Psychiatry 164, 1641-1645 (2007).

24. Jones, E. \& Wessely, S. 'Forward psychiatry' in the military: Its origins and effectiveness. Journal of Traumatic Stress 16, 411-419 (2003).

25. Gersons, B. P. \& Carlier, I. V. Post-traumatic stress disorder: the history of a recent concept. The British Journal of Psychiatry 161, 742-748 (1992).

26. Tate, C. M. et al. Serum brain biomarker level, neurocognitive performance, and self-reported symptom changes in soldiers repeatedly exposed to low-level blast: a breacher pilot study. J Neurotrauma 30, 1620-1630 (2013).

27. Schiehser, D. M., Twamley, E. W. \& Liu, L. The Relationship Between Postconcussive Symptoms and Quality of Life in Veterans With Mild to Moderate Traumatic Brain Injury. J Head Trauma Rehabil (2014).

28. Rao, V. \& Lyketsos, C. Neuropsychiatric Sequelae of Traumatic Brain Injury. Psychosomatics 41, 95-103 (2000).

29. Riggio, S. \& Wong, M. Neurobehavioral sequelae of traumatic brain injury. Mount Sinai Journal of Medicine: A Journal of Translational and Personalized Medicine 76, 163-172 (2009).

30. Sterr, A., Herron, K. A., Hayward, C. \& Montaldi, D. Are mild head injuries as mild as we think? Neurobehavioral concomitants of chronic post-concussion syndrome. BMC Neurology 2006 6:1 6, 1 (2006).

31. Bazarian, J. J. et al. Diffusion tensor imaging detects clinically important axonal damage after mild traumatic brain injury: a pilot study. $J$ Neurotrauma $\mathbf{2 4 , 1 4 4 7 -}$ 1459 (2007).

32. Uomoto, J. M. \& Esselman, P. C. Traumatic brain injury and chronic pain: differential types and rates by head injury severity. Archives of Physical Medicine and Rehabilitation 74, 61-64 (1993). 
33. Couch, J. R. \& Bearss, C. Chronic daily headache in the posttrauma syndrome: relation to extent of head injury. Headache 41, 559-564 (2001).

34. Cantor, J. B. et al. Fatigue after traumatic brain injury and its impact on participation and quality of life. J Head Trauma Rehabil 23, 41-51 (2008).

35. Masel, B. E., Zgaljardic, D. J. \& Forman, J. Post-traumatic hypopituitarism and fatigue. Neuropsychol Rehabil (2015). doi:10.1080/09602011.2015.1125374

36. Englander, J., Bushnik, T., Oggins, J. \& Katznelson, L. Fatigue after traumatic brain injury: Association with neuroendocrine, sleep, depression and other factors. Brain Inj 24, 1379-1388 (2010).

37. Hoffer, M. E., Gottshall, K. R., Moore, R., Balough, B. J. \& Wester, D. Characterizing and treating dizziness after mild head trauma. Otol. Neurotol. 25, 135-138 (2004).

38. Barkhoudarian, G., Hovda, D. A. \& Giza, C. C. The Molecular Pathophysiology of Concussive Brain Injury. Clinics in Sports Medicine 30, 33-48 (2011).

39. Creed, J. A., DiLeonardi, A. M., Fox, D. P., Tessler, A. R. \& Raghupathi, R. Concussive brain trauma in the mouse results in acute cognitive deficits and sustained impairment of axonal function. J Neurotrauma 28, 547-563 (2011).

40. Scheid, R., Walther, K., Guthke, T., Preul, C. \& Cramon, von, D. Y. Cognitive Sequelae of Diffuse Axonal Injury. Arch Neurol 63, 418-424 (2006).

41. Adams, J. H., Graham, D. I. \& Jennett, B. The structural basis of moderate disability after traumatic brain damage. J. Neurol. Neurosurg. Psychiatr. 71, 521-524 (2001).

42. Miles, L. et al. Short-term DTI predictors of cognitive dysfunction in mild traumatic brain injury. Brain Inj 22, 115-122 (2009).

43. Silver, J. M., McAllister, T. W. \& Arciniegas, D. B. Depression and cognitive complaints following mild traumatic brain injury. Am J Psychiatry 166, 653-661 (2009).

44. Iverson, G. L. Outcome from mild traumatic brain injury. Current opinion in psychiatry (2005). doi:10.1016/j.yco.2004.09.001

45. Kim, E. et al. Neuropsychiatric complications of traumatic brain injury: a critical review of the literature (a report by the ANPA Committee on Research). $J$ Neuropsychiatry Clin Neurosci 19, 106-127 (2007).

46. Pontifex, M. B., O'Connor, P. M., Broglio, S. P. \& Hillman, C. H. The association between mild traumatic brain injury history and cognitive control. Neuropsychologia 47, 3210-3216 (2009).

47. Hoge, C. W. et al. Mild Traumatic Brain Injury in U.S. Soldiers Returning from Iraq. N. Engl. J. Med. 358, 453-463 (2008).

48. Bryant, R. A. \& Harvey, A. G. Postconcussive symptoms and posttraumatic stress disorder after mild traumatic brain injury. J. Nerv. Ment. Dis. 187, 302305 (1999).

49. Bryant, R. A. Posttraumatic Stress Disorder and Mild Brain Injury: Controversies, Causes and Consequences. Journal of Clinical and Experimental Neuropsychology 23, 718-728 (2010).

50. Goodrich, G. L. Visual function in patients of a polytrauma rehabilitation center: A descriptive study. JRRD 44, 929-936 (2007). 
51. Magone, M. T., Kwon, E. \& Shin, S. Y. Chronic visual dysfunction after blastinduced mild traumatic brain injury. J Rehabil Res Dev 51, 71-80 (2014).

52. Kaas, J. H. The evolution of the complex sensory and motor systems of the human brain. Brain Res. Bull. 75, 384-390 (2008).

53. Alvarez, T. L. et al. Concurrent vision dysfunctions in convergence insufficiency with traumatic brain injury. Optom Vis Sci 89, 1740-1751 (2012).

54. Brown, J. A., Dalecki, M., Hughes, C., Macpherson, A. K. \& Sergio, L. E. Cognitive-motor integration deficits in young adult athletes following concussion. BMC Sports Sci Med Rehabil 7, 25 (2015).

55. Galetta, K. M. et al. Adding Vision to Concussion Testing. Journal of NeuroOphthalmology 35, 235-241 (2015).

56. Ventura, R. E., Jancuska, J. M., Balcer, L. J. \& Galetta, S. L. Diagnostic Tests for Concussion. Journal of Neuro-Ophthalmology 35, 73-81 (2015).

57. Hunt, A. W., Mah, K., Reed, N., Engel, L. \& Keightley, M. Oculomotor-Based Vision Assessment in Mild Traumatic Brain Injury: A Systematic Review. $J$ Head Trauma Rehabil 1 (2015). doi:10.1097/HTR.0000000000000174

58. Ciuffreda, K. J., Ludlam, D. P., Thiagarajan, P., Yadav, N. K. \& Capo-Aponte, J. Proposed objective visual system biomarkers for mild traumatic brain injury. Mil Med 179, 1212-1217 (2014).

59. Hellerstein, L. F., Freed, S. \& Maples, W. C. Vision profile of patients with mild brain injury. J Am Optom Assoc (1995).

60. Padula, W., Vicci, V., Singman, E. L., Munitz, R. \& Magrun, W. M. in Brain Injury Medicine Principles and Practice (Brain injury ..., 2013).

61. Ciuffreda, K. J. et al. Occurrence of oculomotor dysfunctions in acquired brain injury: A retrospective analysis. Optometry - Journal of the American Optometric Association 78, 155-161 (2007).

62. Thiagarajan, P., Ciuffreda, K. J. \& Ludlam, D. P. Vergence dysfunction in mild traumatic brain injury (mTBI): a review. 31, 456-468 (2011).

63. Ventura, R. E., Balcer, L. J. \& Galetta, S. L. The neuro-ophthalmology of head trauma. The Lancet Neurology 13, 1006-1016 (2014).

64. Kapoor, N. \& Ciuffreda, K. J. Vision Disturbances Following Traumatic Brain Injury. Curr Treat Options Neurol 4, 271-280 (2002).

65. Felten, D. L. \& Shetty, A. N. Netter's atlas of neuroscience. Saunders. (Elsevier, 2010).

66. Green, W. et al. Accommodation in mild traumatic brain injury. J Rehabil Res Dev 47, 183-199 (2010).

67. Chan, R. V. P. \& Trobe, J. D. Spasm of accommodation associated with closed head trauma. Journal of Neuro-Ophthalmology 22, 15-17 (2002).

68. Ciuffreda, K. J. et al. Vision therapy for oculomotor dysfunctions in acquired brain injury: a retrospective analysis. Optometry 79, 18-22 (2008).

69. Szymanowicz, D. et al. Vergence in mild traumatic brain injury: a pilot study. $J$ Rehabil Res Dev 49, 1083-1100 (2012).

70. Cooper, J. \& Jamal, N. Convergence insufficiency-a major review. Optometry 83, 137-158 (2012). 
71. Al-Qurainy, I. A. Convergence insufficiency and failure of accommodation following midfacial trauma. British Journal of Oral and Maxillofacial Surgery 33, 71-75 (1995).

72. Haines, D. E. ... Neuroscience for Basic and Clinical Applications. (2013).

73. Mays, L. E. \& Porter, J. D. Neural control of vergence eye movements: activity of abducens and oculomotor neurons. J. Neurophysiol. 52, 743-761 (1984).

74. Kraus, M. F. et al. Oculomotor Function in Chronic Traumatic Brain Injury. Cognitive and Behavioral Neurology 20, 170 (2007).

75. Berman, R. A. et al. Cortical networks subserving pursuit and saccadic eye movements in humans: an FMRI study. Hum Brain Mapp 8, 209-225 (1999).

76. $\mathrm{Xu}, \mathrm{G} .-\mathrm{Q}$. et al. Visuospatial attention deficit in patients with local brain lesions. Brain Res. 1322, 153-159 (2010).

77. Cockerham, G. C. et al. Eye and visual function in traumatic brain injury. $J$ Rehabil Res Dev 46, 811-818 (2009).

78. Digre, K. B. \& Brennan, K. C. Shedding Light on Photophobia. Journal of Neuro-Ophthalmology 32, 68-81 (2012).

79. Matynia, A. et al. Intrinsically photosensitive retinal ganglion cells are the primary but not exclusive circuit for light aversion. Exp. Eye Res. 105, 60-69 (2012).

80. Noseda, R., Constandil, L., Bourgeais, L., Chalus, M. \& Villanueva, L. Changes of meningeal excitability mediated by corticotrigeminal networks: a link for the endogenous modulation of migraine pain. J Neurosci 30, 14420-14429 (2010).

81. Okamoto, K., Thompson, R., Tashiro, A., Chang, Z. \& Bereiter, D. A. Bright light produces Fos-positive neurons in caudal trigeminal brainstem.

Neuroscience 160, 858-864 (2009).

82. Cantu, R. C. Chronic traumatic encephalopathy in the National Football League. Neurosurgery 61, 223-225 (2007).

83. Saatman, K. E. et al. Classification of traumatic brain injury for targeted therapies. in 25, 719-738 (2008).

84. Morey, R. A. et al. Effects of chronic mild traumatic brain injury on white matter integrity in Iraq and Afghanistan war veterans. Hum Brain Mapp 34, 2986-2999 (2013).

85. Adams, J. H. et al. Diffuse axonal injury in head injury: Definition, diagnosis and grading. Histopathology 15, 49-59 (1989).

86. Meythaler, J. M., Peduzzi, J. D., Eleftheriou, E. \& Novack, T. A. Current concepts: Diffuse axonal injury-associated traumatic brain injury. Archives of Physical Medicine and Rehabilitation 82, 1461-1471 (2001).

87. Maxwell, W. L., Povlishock, J. T. \& Graham, D. L. A mechanistic analysis of nondisruptive axonal injury: a review. J Neurotrauma 14, 419-440 (1997).

88. Tang-Schomer, M. D., Patel, A. R., Baas, P. W. \& Smith, D. H. Mechanical breaking of microtubules in axons during dynamic stretch injury underlies delayed elasticity, microtubule disassembly, and axon degeneration. The FASEB Journal 24, 1401-1410 (2010).

89. Smith, D. H., Wolf, J. A., Lusardi, T. A., Lee, V. M. \& Meaney, D. F. High tolerance and delayed elastic response of cultured axons to dynamic stretch injury. J Neurosci 19, 4263-4269 (1999). 
90. Gennarelli, T. A. et al. Directional Dependence of Axonal Brain Injury due to Centroidal and Non-Centroidal Acceleration. STAPP 1, 872197 (1987).

91. Margulies, S. S., Thibault, L. E. \& Gennarelli, T. A. Physical model simulations of brain injury in the primate. Journal of Biomechanics 23, 823-836 (1990).

92. Farkas, O. \& Povlishock, J. T. in Neurotrauma: New Insights into Pathology and Treatment 161, 43-59 (Elsevier, 2007).

93. Baldwin, S. A., Fugaccia, I., Brown, D. R., Brown, L. V. \& Scheff, S. W. Bloodbrain barrier breach following cortical contusion in the rat. J. Neurosurg. 85, 476-481 (1996).

94. Farkas, O., Lifshitz, J. \& Povlishock, J. T. Mechanoporation Induced by Diffuse Traumatic Brain Injury: An Irreversible or Reversible Response to Injury? $J$ Neurosci 26, 3130-3140 (2006).

95. Blaylock, R. L. Immunology primer for neurosurgeons and neurologists part 2: Innate brain immunity. Surg Neurol Int 4, 118 (2013).

96. Katayama, Y., Becker, D. P., Tamura, T. \& Hovda, D. A. Massive increases in extracellular potassium and the indiscriminate release of glutamate following concussive brain injury. J. Neurosurg. 73, 889-900 (1990).

97. Kawamata, T., Katayama, Y., Hovda, D. A., Yoshino, A. \& Becker, D. P. Lactate accumulation following concussive brain injury: the role of ionic fluxes induced by excitatory amino acids. Brain Res. 674, 196-204 (1995).

98. Lifshitz, J., Sullivan, P. G., Hovda, D. A., Wieloch, T. \& McIntosh, T. K. Mitochondrial damage and dysfunction in traumatic brain injury. Mitochondrion 4, 705-713 (2004).

99. Ankeny, D. P. \& Popovich, P. G. Mechanisms and implications of adaptive immune responses after traumatic spinal cord injury. Neuroscience 158, 1112 1121 (2009).

100. Trauma in Greenfield's Neuropathology Vol. 1 (eds. Love, S., Louis, D. N. \& Ellison, D. W.) 1, 733-812 (Edward Arnold Publishers Ltd.).

101. Kreutzberg, G. W. Microglia: a sensor for pathological events in the CNS. Trends in Neurosciences (1996).

102. Ben Borgens, R. \& Liu-Snyder, P. Understanding Secondary Injury. The Quarterly Review of Biology 87, 89-127 (2012).

103. Karman, J., Ling, C., Sandor, M. \& Fabry, Z. Initiation of Immune Responses in Brain Is Promoted by Local Dendritic Cells. J. Immunol. 173, 2353-2361 (2004).

104. Jones, T. B. Lymphocytes and autoimmunity after spinal cord injury. Exp. Neurol. 258, 78-90 (2014).

105. Lucas, S. M., Rothwell, N. J. \& Gibson, R. M. The role of inflammation in CNS injury and disease. Br. J. Pharmacol. 147, S232-S240 (2006).

106. Holmin, S. \& Höjeberg, B. In situ detection of intracerebral cytokine expression after human brain contusion. Neuroscience Letters 369, 108-114 (2004).

107. Hausmann, E. H. S. et al. Selective chemokine mRNA expression following brain injury. Brain Res. 788, 49-59 (1998).

108. Sebire, G. et al. In vitro production of IL-6, IL-1 beta, and tumor necrosis factoralpha by human embryonic microglial and neural cells. J. Immunol. 150, 15171523 (1993). 
109. Engelhardt, B. \& Coisne, C. Fluids and barriers of the CNS establish immune privilege by confining immune surveillance to a two-walled castle moat surrounding the CNS castle. Fluids Barriers CNS (2011).

110. Carlos, T. M., Clark, R. S., Franicola-Higgins, D., Schiding, J. K. \& Kochanek, P. M. Expression of endothelial adhesion molecules and recruitment of neutrophils after traumatic brain injury in rats. J. Leukoc. Biol. 61, 279-285 (1997).

111. Israelsson, C. et al. Distinct cellular patterns of upregulated chemokine expression supporting a prominent inflammatory role in traumatic brain injury. $J$ Neurotrauma 25, 959-974 (2008).

112. Banchereau, J. \& Steinman, R. M. Dendritic cells and the control of immunity. Nature (1998).

113. Kelso, M. L. \& Gendelman, H. E. Bridge between neuroimmunity and traumatic brain injury. Curr. Pharm. Des. 20, 4284-4298 (2014).

114. Streit, W. J. Microglia as neuroprotective, immunocompetent cells of the CNS. Glia 40, 133-139 (2002).

115. Hanisch, U.-K. \& Kettenmann, H. Microglia: active sensor and versatile effector cells in the normal and pathologic brain. Nat Neurosci 10, 1387-1394 (2007).

116. Mantovani, A. et al. The chemokine system in diverse forms of macrophage activation and polarization. Trends in Immunology 25, 677-686 (2004).

117. Durafourt, B. A. et al. Comparison of polarization properties of human adult microglia and blood-derived macrophages. Glia 60, 717-727 (2012).

118. Glenn, J. A., Ward, S. A., Stone, C. R., Booth, P. L. \& Thomas, W. E. Characterisation of ramified microglial cells: detailed morphology, morphological plasticity and proliferative capability. Journal of Anatomy 180, 109-118 (1992).

119. Nimmerjahn, A., Kirchhoff, F. \& Helmchen, F. Resting Microglial Cells Are Highly Dynamic Surveillants of Brain Parenchyma in Vivo. Science 308, 13141318 (2005).

120. Loane, D. J. \& Byrnes, K. R. Role of microglia in neurotrauma. Neurotherapeutics 7, 366-377 (2010).

121. Kigerl, K. A. et al. Identification of Two Distinct Macrophage Subsets with Divergent Effects Causing either Neurotoxicity or Regeneration in the Injured Mouse Spinal Cord. J Neurosci 29, 13435-13444 (2009).

122. Aguzzi, A., Barres, B. A. \& Bennett, M. L. Microglia: scapegoat, saboteur, or something else? Science 339, 156-161 (2013).

123. Wang, G. et al. Microglia/macrophage polarization dynamics in white matter after traumatic brain injury. J. Cereb. Blood Flow Metab. 33, 1864-1874 (2013).

124. Prinz, M. \& Priller, J. Microglia and brain macrophages in the molecular age: from origin to neuropsychiatric disease. Nat. Rev. Neurosci. 15, 300-312 (2014).

125. Babcock, A. A., Kuziel, W. A., Rivest, S. \& Owens, T. Chemokine Expression by Glial Cells Directs Leukocytes to Sites of Axonal Injury in the CNS. $J$ Neurosci 23, 7922-7930 (2003).

126. Pinteaux Jones, F. et al. Myelin-induced microglial neurotoxicity can be controlled by microglial metabotropic glutamate receptors. J. Neurochem. 106, 442-454 (2008). 
127. Hu, X. et al. Microglia/macrophage polarization dynamics reveal novel mechanism of injury expansion after focal cerebral ischemia. Stroke 43, 30633070 (2012).

128. David, S. \& Kroner, A. Repertoire of microglial and macrophage responses after spinal cord injury. Nat. Rev. Neurosci. 12, 388-399 (2011).

129. Miron, V. E. et al. M2 microglia and macrophages drive oligodendrocyte differentiation during CNS remyelination. Nat Neurosci 16, 1211-1218 (2013).

130. Li, L., Lu, J., Tay, S. S. W., Moochhala, S. M. \& He, B. P. The function of microglia, either neuroprotection or neurotoxicity, is determined by the equilibrium among factors released from activated microglia in vitro. Brain Res. 1159, 8-17 (2007).

131. Franco, R. \& Fernández-Suárez, D. Alternatively activated microglia and macrophages in the central nervous system. Prog. Neurobiol. 131, 65-86 (2015).

132. Lyons, A., Griffin, R. J., Costelloe, C. E., Clarke, R. M. \& Lynch, M. A. IL-4 attenuates the neuroinflammation induced by amyloid- $\beta$ in vivo and in vitro. $J$. Neurochem. 101, 771-781 (2007).

133. Fang, K. M., Yang, C. S., Sun, S. H. \& Tzeng, S. F. Microglial phagocytosis attenuated by short-term exposure to exogenous ATP through P2X7 receptor action. J. Neurochem. 111, 1225-1237 (2009).

134. Johnson, V. E. et al. Inflammation and white matter degeneration persist for years after a single traumatic brain injury. Brain 136, 28-42 (2013).

135. Cernak, I. Animal models of head trauma. Neurotherapeutics 2, 410-422 (2005).

136. Vorwerk, C. K., Zurakowski, D., McDermott, L. M., Mawrin, C. \& Dreyer, E. B. Effects of axonal injury on ganglion cell survival and glutamate homeostasis. Brain Res. Bull. 62, 485-490 (2004).

137. Qu, J. \& Jakobs, T. C. The Time Course of Gene Expression during Reactive Gliosis in the Optic Nerve. PLoS ONE 8, e67094 (2013).

138. Thompson, H. J. et al. Lateral fluid percussion brain injury: a 15-year review and evaluation. J Neurotrauma 22, 42-75 (2005).

139. Cheng, C. \& Povlishock, J. T. The effect of traumatic brain injury on the visual system: a morphologic characterization of reactive axonal change. $J$

Neurotrauma (1988). doi:10.1089/neu.1988.5.47

140. Williams, A. J., Hartings, J. A., Lu, X.-C. M., Rolli, M. L. \& Tortella, F. C. Penetrating ballistic-like brain injury in the rat: differential time courses of hemorrhage, cell death, inflammation, and remote degeneration. $J$ Neurotrauma 23, 1828-1846 (2006).

141. Lighthall, J. W. Controlled cortical impact: a new experimental brain injury model. J Neurotrauma (1988). doi:10.1089/neu.1988.5.1

142. Marmarou, A. et al. A new model of diffuse brain injury in rats. Part I: Pathophysiology and biomechanics. J. Neurosurg. 80, 291-300 (1994).

143. Foda, M. A. \& Marmarou, A. A new model of diffuse brain injury in rats. Part II: Morphological characterization. J. Neurosurg. 80, 301-313 (1994).

144. Tzekov, R. et al. Repetitive mild traumatic brain injury causes optic nerve and retinal damage in a mouse model. J Neuropathol Exp Neurol 73, 345-361 (2014). 
145. Nakagawa, A. et al. Mechanisms of Primary Blast-Induced Traumatic Brain Injury: Insights from Shock-Wave Research. J Neurotrauma 28, 1101-1119 (2011).

146. Prevost, T. P., Balakrishnan, A., Suresh, S. \& Socrate, S. Biomechanics of brain tissue. Acta Biomater 7, 83-95 (2011).

147. Nyein, M. K. et al. In silico investigation of intracranial blast mitigation with relevance to military traumatic brain injury. Proc Natl Acad Sci USA 107, 20703-20708 (2010).

148. Taylor, P. A. \& Ford, C. C. Simulation of blast-induced early-time intracranial wave physics leading to traumatic brain injury. J Biomech Eng 131, 061007 (2009).

149. Peskind, E. R., Brody, D., Cernak, I., McKee, A. \& Ruff, R. L. Military- and sports-related mild traumatic brain injury: clinical presentation, management, and long-term consequences. J Clin Psychiatry 74, 180-8- quiz 188 (2013).

150. Ruff, R. L., Riechers, R. G., II, Wang, X.-F., Piero, T. \& Ruff, S. S. A casecontrol study examining whether neurological deficits and PTSD in combat veterans are related to episodes of mild TBI. BMJ Open 2, e000312 (2012).

151. Lange, R. T. et al. Neuropsychological Outcome from Blast versus Non-blast: Mild Traumatic Brain Injury in U.S. Military Service Members. J Int Neuropsychol Soc 18, 595-605 (2012).

152. Dutca, L. M. et al. Early detection of subclinical visual damage after blastmediated TBI enables prevention of chronic visual deficit by treatment with P7C3-S243. Investigative Ophthalmology \& Visual Science 55, 8330-8341 (2014).

153. Koliatsos, V. E. et al. A mouse model of blast injury to brain: initial pathological, neuropathological, and behavioral characterization. J Neuropathol Exp Neurol 70, 399-416 (2011).

154. Wang, H.-C. H. et al. Pathophysiology of blast-induced ocular trauma with apoptosis in the retina and optic nerve. Mil Med 179, 34-40 (2014).

155. Wang, J., Fox, M. A. \& Povlishock, J. T. Diffuse Traumatic Axonal Injury in the Optic Nerve Does Not Elicit Retinal Ganglion Cell Loss. J Neuropathol Exp Neurol 72, 768-781 (2013).

156. Janak, J. C., Cooper, D. B. \& Bowles, A. O. Completion of Multidisciplinary Treatment for Persistent Postconcussive Symptoms Is Associated With Reduced Symptom Burden. J Head Trauma Rehabil (2015).

157. McCrory, P. et al. Consensus statement on concussion in sport: the 4th International Conference on Concussion in Sport held in Zurich, November 2012. in 216, e55-71 (2013).

158. Silverberg, N. D. \& Iverson, G. L. Is rest after concussion' the best medicine?’: recommendations for activity resumption following concussion in athletes, civilians, and military service members. J Head Trauma Rehabil (2013).

159. Leddy, J. J. et al. Exercise treatment for postconcussion syndrome: a pilot study of changes in functional magnetic resonance imaging activation, physiology, and symptoms. J Head Trauma Rehabil 28, 241-249 (2013).

160. Skolnick, B. E. et al. A clinical trial of progesterone for severe traumatic brain injury. N. Engl. J. Med. 371, 2467-2476 (2014). 
161. Chang, L. C., Raty, S. R., Ortiz, J. \& Bailard, N. S. The emerging use of ketamine for anesthesia and sedation in traumatic brain injuries. CNS Neuroscience \& Therapeutics (2013). doi:10.1111/cns.12077

162. Gultekin, R. et al. Pharmacological interventions in traumatic brain injury: Can we rely on systematic reviews for evidence? Injury 47, 516-524 (2016).

163. Crossley, S., Reid, J., McLatchie, R., Hayton, J. \& Clark, C. A systematic review of therapeutic hypothermia for adult patients following traumatic brain injury. Crit Care (2014).

164. Chatzipanteli, K., Wada, K., Busto, R. \& Dietrich, W. D. Effects of Moderate Hypothermia on Constitutive and Inducible Nitric Oxide Synthase Activities After Traumatic Brain Injury in the Rat. J. Neurochem. 72, 2047-2052 (1999).

165. Koski, L. et al. Noninvasive brain stimulation for persistent postconcussion symptoms in mild traumatic brain injury. J Neurotrauma 32, 38-44 (2015).

166. Ludlam, D. P. \& Kapoor, O. N. Clinical oculomotor training in traumatic brain injury. (2009).

167. Tassinari, J. D. Binasal occlusion. (J Behav Optom, 1990).

168. Hudac, C. M., Kota, S., Nedrow, J. L. \& Molfese, D. L. Neural mechanisms underlying neurooptometric rehabilitation following traumatic brain injury. Eye and Brain (2012).

169. Yadav, N. K. \& Ciuffreda, K. J. Effect of binasal occlusion (BNO) and base-in prisms on the visual-evoked potential (VEP) in mild traumatic brain injury (mTBI). Brain Inj 28, 1568-1580 (2014).

170. Tanielian, T., Haycox, L. H., Schell, T. L. \& Marshall, G. N. Invisible Wounds of War. Summary and Recommendations for Addressing Psychological and Cognitive Injuries. (2008).

171. Dougherty, A. L., MacGregor, A. J., Han, P. P., Heltemes, K. J. \& Galarneau, M. R. Visual dysfunction following blast-related traumatic brain injury from the battlefield. Brain Inj 25, 8-13 (2011).

172. Goodrich, G. L., Flyg, H. M., Kirby, J. E., Chang, C.-Y. \& Martinsen, G. L. Mechanisms of TBI and visual consequences in military and veteran populations. Optom Vis Sci 90, 105-112 (2013).

173. Petras, J. M., Bauman, R. A. \& Elsayed, N. M. Visual system degeneration induced by blast overpressure. Toxicology 121, 41-49 (1997).

174. Frick, K. D. Costs of Military Eye Injury, Vision Impairment, and Related Blindnessand Vision Dysfunction Associated with Traumatic Brain Injury (TBI) without Eye Injury. 1-16 (2012). at $<$ http://www.eyeresearch.org/pdf/costofmilitaryblindness.pdf $>$

175. Cernak, I. The importance of systemic response in the pathobiology of blastinduced neurotrauma. Front. Neur. 1, 151 (2010).

176. Hines-Beard, J. et al. A mouse model of ocular blast injury that induces closed globe anterior and posterior pole damage. Exp. Eye Res. 99, 63-70 (2012).

177. Bricker-Anthony, C., Hines-Beard, J. \& Rex, T. S. Molecular changes and vision loss in a mouse model of closed-globe blast trauma. Investigative Ophthalmology \& Visual Science 55, 4853-4862 (2014). 
178. Bricker-Anthony, C. \& Rex, T. S. Neurodegeneration and Vision Loss after Mild Blunt Trauma in the C57Bl/6 and DBA/2J Mouse. PLoS ONE 10, e 0131921 (2015).

179. Guley, N. H. et al. A Novel Closed-Head Model of Mild Traumatic Brain Injury Using Focal Primary Overpressure Blast to the Cranium in Mice. J Neurotrauma 33, 403-422 (2016).

180. Heldt, S. A. et al. A novel closed-head model of mild traumatic brain injury caused by primary overpressure blast to the cranium produces sustained emotional deficits in mice. Front. Neur. 5, 2 (2014).

181. Reiner, A. et al. Motor, visual and emotional deficits in mice after closed-head mild traumatic brain injury are alleviated by the novel CB2 inverse agonist SMM-189. Int J Mol Sci 16, 758-787 (2015).

182. Marmor, M. F. Contrast sensitivity versus visual acuity in retinal disease. $\mathrm{Br} J$ Ophthalmol 70, 553-559 (1986).

183. Douglas, R. M. et al. Independent visual threshold measurements in the two eyes of freely moving rats and mice using a virtual-reality optokinetic system. Vis.

Neurosci. 22, 677-684 (2005).

184. Hall, E. D. et al. Spatial and temporal characteristics of neurodegeneration after controlled cortical impact in mice: more than a focal brain injury. $J$ Neurotrauma 22, 252-265 (2005).

185. Del Mar, N. et al. A novel closed-body model of spinal cord injury caused by high-pressure air blasts produces extensive axonal injury and motor impairments. Exp. Neurol. 271, 53-71 (2015).

186. Howlett, A. C. et al. International Union of Pharmacology. XXVII. Classification of Cannabinoid Receptors. Pharmacol Rev 54, 161-202 (2002).

187. Pertwee, R. G. et al. International Union of Basic and Clinical Pharmacology. LXXIX. Cannabinoid Receptors and Their Ligands: Beyond CB1 and CB2. Pharmacol Rev 62, 588-631 (2010).

188. Pertwee, R. G. Neuropharmacology and therapeutic potential of cannabinoids. Addiction Biology 5, 37-46 (2000).

189. Galve-Roperh, I. et al. Cannabinoid receptor signaling in progenitor/stem cell proliferation and differentiation. Progress in Lipid Research 52, 633-650 (2013).

190. Kenakin, T. Inverse, protean, and ligand-selective agonism: matters of receptor conformation. The FASEB Journal (2001).

191. Landsman, R. S. et al. AM630 is an inverse agonist at the human cannabinoid CB1 receptor. Life Sciences 62, PL109-PL113 (1998).

192. Porter, A. C. \& Felder, C. C. The endocannabinoid nervous system. Pharmacology \& Therapeutics 90, 45-60 (2001).

193. Howlett, A. C. et al. Endocannabinoid tone versus constitutive activity of cannabinoid receptors. Br. J. Pharmacol. 163, 1329-1343 (2011).

194. Stella, N. Cannabinoid and cannabinoid-like receptors in microglia, astrocytes, and astrocytomas. Glia 58, 1017-1030 (2010).

195. Basu, S. \& Dittel, B. N. Unraveling the complexities of cannabinoid receptor 2 (CB2) immune regulation in health and disease. Immunol. Res. 51, 26-38 (2011).

196. Chiurchiù, V., Battistini, L. \& Maccarrone, M. Endocannabinoid signaling in innate and adaptive immunity. Immunology (2015). doi:10.1111/imm.12441 
197. Fowler, C. J. Plant-derived, synthetic and endogenous cannabinoids as neuroprotective agents. Brain Research Reviews 41, 26-43 (2003).

198. Ben Amar, M. Cannabinoids in medicine: A review of their therapeutic potential. J Ethnopharmacol 105, 1-25 (2006).

199. Carlisle, S. J., Marciano-Cabral, F., Staab, A., Ludwick, C. \& Cabral, G. A. Differential expression of the $\mathrm{CB} 2$ cannabinoid receptor by rodent macrophages and macrophage-like cells in relation to cell activation. Int. Immunopharmacol. 2, 69-82 (2002).

200. Maresz, K., Carrier, E. J., Ponomarev, E. D., Hillard, C. J. \& Dittel, B. N. Modulation of the cannabinoid CB2 receptor in microglial cells in response to inflammatory stimuli. J. Neurochem. 95, 437-445 (2005).

201. Krishnan, G. \& Chatterjee, N. Anandamide rescues retinal barrier properties in Müller glia through nitric oxide regulation. Neuroscience 284, 536-545 (2015).

202. Pertwee, R. G. The diverse CB 1 and CB 2receptor pharmacology of three plant cannabinoids: $\Delta$ 9-tetrahydrocannabinol, cannabidiol and $\Delta 9$ tetrahydrocannabivarin. Br. J. Pharmacol. 153, 199-215 (2008).

203. Carrier, E. J. et al. Cultured rat microglial cells synthesize the endocannabinoid 2-arachidonylglycerol, which increases proliferation via a CB2 receptordependent mechanism. Mol. Pharmacol. 65, 999-1007 (2004).

204. Palazuelos, J. et al. Microglial CB2 cannabinoid receptors are neuroprotective in Huntington's disease excitotoxicity. Brain 132, 3152-3164 (2009).

205. Lunn, C. A. et al. A novel cannabinoid peripheral cannabinoid receptor-selective inverse agonist blocks leukocyte recruitment in vivo. J. Pharmacol. Exp. Ther. 316, 780-788 (2006).

206. Ueda, Y., Miyagawa, N., Matsui, T., Kaya, T. \& Iwamura, H. Involvement of cannabinoid $\mathrm{CB} 2$ receptor-mediated response and efficacy of cannabinoid CB2 receptor inverse agonist, JTE-907, in cutaneous inflammation in mice. European Journal of Pharmacology 520, 164-171 (2005).

207. Bhattacharjee, H., Gurley, S. N. \& Moore, B. M. Design and synthesis of novel tri-aryl CB2 selective cannabinoid ligands. Bioorg. Med. Chem. Lett. 19, 16911693 (2009).

208. Fujinaga, M. et al. Bioorganic \& Medicinal Chemistry Letters. Bioorg. Med. Chem. Lett. 20, 1565-1568 (2010).

209. Gurley, S. N. Synthesis of novel cannabinoid ligands and their use as anti-glioma and anti-inflammatory agents. (2010).

210. Kimble, T. D. H., Fitzgerald, M. E. C. \& Reiner, A. Sustained upregulation of glial fibrillary acidic protein in Müller cells in pigeon retina following disruption of the parasympathetic control of choroidal blood flow. Exp. Eye Res. 83, 10171030 (2006).

211. Soto, I. et al. Retinal ganglion cell loss in a rat ocular hypertension model is sectorial and involves early optic nerve axon loss. Investigative Ophthalmology \& Visual Science 52, 434-441 (2011).

212. Nadal-Nicola s, F. M. et al. Brn3a as a Marker of Retinal Ganglion Cells: Qualitative and Quantitative Time Course Studies in Naïve and Optic NerveInjured Retinas. Investigative Ophthalmology \& Visual Science 50, 3860-9 (2009). 
213. Surgucheva, I., Weisman, A. D., Goldberg, J. L., Shnyra, A. \& Surguchov, A. Gamma-synuclein as a marker of retinal ganglion cells. Mol. Vis. 14, 1540-1548 (2008).

214. Tanimoto, N. et al. Vision tests in the mouse: Functional phenotyping with electroretinography. Front Biosci (Landmark Ed) 14, 2730-2737 (2009).

215. Perlman, I. The Electroretogram: ERG. Webvision: The Organization of the Retina and Visual ... (2011).

216. Sarno, S. et al. Electrophysiological correlates of visual impairments after traumatic brain injury. Vision Res. 40, 3029-3038 (2000).

217. Freed, S. \& Hellerstein, L. F. Visual electrodiagnostic findings in mild traumatic brain injury. Brain Inj 11, 25-36 (1997).

218. Presley, C. et al. Preclinical evaluation of SMM-189, a cannabinoid receptor 2specific inverse agonist. Pharmacol Res Perspect 3, n/a-n/a (2015).

219. Fujinaga, M. et al. Radiosynthesis of novel carbon-11-labeled triaryl ligands for cannabinoid-type 2 receptor. Bioorg. Med. Chem. Lett. 20, 1565-1568 (2010).

220. McCloy, R. A. et al. Partial inhibition of Cdk1 in G 2 phase overrides the SAC and decouples mitotic events. Cell Cycle 13, 1400-1412 (2014).

221. Zeng, X. X., Ng, Y. K. \& Ling, E. A. Neuronal and microglial response in the retina of streptozotocin-induced diabetic rats. Vis. Neurosci. 17, 463-471 (2000).

222. Gaucher, D. et al. Microglial changes occur without neural cell death in diabetic retinopathy. Vision Res. 47, 612-623 (2007).

223. Schnitzer, J. \& Scherer, J. Microglial cell responses in the rabbit retina following transection of the optic nerve. J. Comp. Neurol. 302, 779-791 (1990).

224. Thanos, S. The Relationship of Microglial Cells to Dying Neurons During Natural Neuronal Cell Death and Axotomy-induced Degeneration of the Rat Retina. Eur. J. Neurosci. 3, 1189-1207 (1991).

225. Wang, M., Ma, W., Zhao, L., Fariss, R. N. \& Wong, W. T. Adaptive Müller cell responses to microglial activation mediate neuroprotection and coordinate inflammation in the retina. J Neuroinflammation 8, 173 (2011).

226. Thanos, S. Sick photoreceptors attract activated microglia from the ganglion cell layer: a model to study the inflammatory cascades in rats with inherited retinal dystrophy. Brain Res. 588, 21-28 (1992).

227. Lunn, C. A. et al. Biology and therapeutic potential of cannabinoid CB2 receptor inverse agonists. Br. J. Pharmacol. 153, 226-239 (2008).

228. Bosco, A., Steele, M. R. \& Vetter, M. L. Early microglia activation in a mouse model of chronic glaucoma. J. Comp. Neurol. 519, 599-620 (2011).

229. Madeira, M. H., Boia, R., Santos, P. F., Ambrósio, A. F. \& Santiago, A. R. Contribution of microglia-mediated neuroinflammation to retinal degenerative diseases. Mediators Inflamm. 2015, 673090-15 (2015).

230. Bringmann, A. \& Wiedemann, P. Müller glial cells in retinal disease. Ophthalmologica (2011). doi:10.1159/000328979

231. Hiscott, P. S., Grierson, I., Trombetta, C. J. \& Rahi, A. H. Retinal and epiretinal glia--an immunohistochemical study. British journal of ... (1984).

doi:10.1136/bjo.68.10.698 
232. Ekström, P., Sanyal, S. \& Narfström, K. Accumulation of glial fibrillary acidic protein in Müller radial glia during retinal degeneration. ... \& visual science (1988).

233. Ramírez, J. M., Ramírez, A. I., Salazar, J. J., de Hoz, R. \& Triviño, A. Changes of Astrocytes in Retinal Ageing and Age-related Macular Degeneration. Exp. Eye Res. 73, 601-615 (2001).

234. Humphrey, M. F., Constable, I. J., Chu, Y. \& Wiffen, S. A quantitative study of the lateral spread of Müller cell responses to retinal lesions in the rabbit. $J$. Comp. Neurol. 334, 545-558 (1993).

235. Humphrey, M. F. \& Moore, S. R. Microglial responses to focal lesions of the rabbit retina: Correlation with neural and macroglial reactions. Glia 16, 325-341 (1996).

236. Greferath, U. et al. Inner retinal change in a novel rd1-FTL mouse model of retinal degeneration. Front. Cell. Neurosci. 9, 293 (2015).

237. Cuenca, N. et al. Cellular responses following retinal injuries and therapeutic approaches for neurodegenerative diseases. Prog Retin Eye Res 43C, 17-75 (2014).

238. Verbin, H. L. et al. Optic Nerve Transection in Monkeys May Result in Secondary Degeneration of Retinal Ganglion Cells. Investigative Ophthalmology \& Visual Science 42, 975-982 (2001).

239. Yoles, E. \& Schwartz, M. Degeneration of Spared Axons Following Partial White Matter Lesion: Implications for Optic Nerve Neuropathies. Exp. Neurol. 153, 1-7 (1998).

240. Bradley, J. L. et al. Ultra-high-resolution optical coherence tomographic findings in commotio retinae. Arch. Ophthalmol. 129, 107-108 (2011).

241. Pham, T. Q., Chua, B., Gorbatov, M. \& Mitchell, P. Optical coherence tomography findings of acute traumatic maculopathy following motor vehicle accident. American Journal of Ophthalmology 143, 348-350 (2007).

242. Flatter, J. A. et al. Outer retinal structure after closed-globe blunt ocular trauma. Retina (Philadelphia, Pa.) 34, 2133-2146 (2014).

243. Shanahan, D. F. Human tolerance and crash survivability. Pathological Aspects and Associate Biodynamics in Aircraft Accident Investigation (2004).

244. Bosco, A. et al. Glial coverage in the optic nerve expands in proportion to optic axon loss in chronic mouse glaucoma. Exp. Eye Res. (2016). doi:10.1016/j.exer.2016.01.014

245. Burgoyne, C. F. A biomechanical paradigm for axonal insult within the optic nerve head in aging and glaucoma. Exp. Eye Res. 93, 120-132 (2011).

246. Levin, L. A., Beck, R. W., Joseph, M. P., Seiff, S. \& Kraker, R. The treatment of traumatic optic neuropathy. Ophthalmology 106, 1268-1277 (1999).

247. Sun, D., Lye-Barthel, M., Masland, R. H. \& Jakobs, T. C. The morphology and spatial arrangement of astrocytes in the optic nerve head of the mouse. J. Comp. Neurol. 516, 1-19 (2009).

248. Stafford, C. A., Shehab, S. A. S., Nona, S. N. \& Cronly Dillon, J. R. Expression of glial fibrillary acidic protein (GFAP) in goldfish optic nerve following injury. Glia 3, 33-42 (1990). 
249. Dräger, U. C. Birth dates of retinal ganglion cells giving rise to the crossed and uncrossed optic projections in the mouse. Proc. R. Soc. Lond., B, Biol. Sci. 224, 57-77 (1985).

250. Fitch, M. T. \& Silver, J. CNS injury, glial scars, and inflammation: Inhibitory extracellular matrices and regeneration failure. Exp. Neurol. 209, 294-301 (2008).

251. Kerschensteiner, M., Schwab, M. E., Lichtman, J. W. \& Misgeld, T. In vivo imaging of axonal degeneration and regeneration in the injured spinal cord. Nature Medicine 11, 572-577 (2005).

252. Büki, A. \& Povlishock, J. T. All roads lead to disconnection?--Traumatic axonal injury revisited. Acta Neurochir (Wien) 148, 181-93; discussion 193-4 (2006).

253. Saxena, S. \& Caroni, P. Mechanisms of axon degeneration: From development to disease. Prog. Neurobiol. 83, 174-191 (2007).

254. Chung, R. S. et al. Mild axonal stretch injury in vitro induces a progressive series of neurofilament alterations ultimately leading to delayed axotomy. $J$

Neurotrauma 22, 1081-1091 (2005).

255. Swanson, J. A. \& Hoppe, A. D. The coordination of signaling during Fc receptor-mediated phagocytosis. J. Leukoc. Biol. 76, 1093-1103 (2004).

256. Gazi, U. \& Martinez-Pomares, L. Influence of the mannose receptor in host immune responses. Immunobiology 214, 554-561 (2009).

257. Lee, S. J. et al. Mannose receptor-mediated regulation of serum glycoprotein homeostasis. Science 295, 1898-1901 (2002).

258. Mosser, D. M. \& Edwards, J. P. Exploring the full spectrum of macrophage activation. Nat. Rev. Immunol. (2008). doi:10.1038/nri2448

259. Hanisch, U.-K. Functional diversity of microglia - how heterogeneous are they to begin with? Front. Cell. Neurosci. 7, 65 (2013).

260. Peferoen, L. A. N. et al. Activation Status of Human Microglia Is Dependent on Lesion Formation Stage and Remyelination in Multiple Sclerosis. J Neuropathol Exp Neurol 74, 48-63 (2015).

261. Xu, F. et al. Microglial polarization dynamics in dorsal spinal cord in the early stages following chronic sciatic nerve damage. Neuroscience Letters 617, 6-13 (2016).

262. Godbout, J. P. et al. Exaggerated neuroinflammation and sickness behavior in aged mice following activation of the peripheral innate immune system. FASEB J. 19, 1329-1331 (2005).

263. Cherry, J. D., Olschowka, J. A. \& O'Banion, M. K. Are "resting" microglia more "m2"? Front Immunol 5, 594 (2014).

264. Perlman, I. The Electroretinogram: ERG by Ido Perlman. Webvision: The Organization of the Retina and ... (2015).

265. Mohan, K., Kecova, H., Hernandez-Merino, E., Kardon, R. H. \& Harper, M. M. Retinal ganglion cell damage in an experimental rodent model of blast-mediated traumatic brain injury. Investigative Ophthalmology \& Visual Science 54, 34403450 (2013).

266. Du, T., Ciuffreda, K. J. \& Kapoor, N. Elevated dark adaptation thresholds in traumatic brain injury. Brain Inj 19, 1125-1138 (2005). 
267. Newman, E. A., Fishman, G. A. \& Frishman, L. J. in Principles and Practice of Clinical Electrophysiology of Vision (eds. Heckenlively, J. R. \& Arden, G. B.) (Principle and ..., 1991).

268. Lei, B. \& Perlman, I. The contributions of voltage- and time-dependent potassium conductances to the electroretinogram in rabbits. Vis. Neurosci. 16, 743-754 (1999).

269. Sillman, A. J. Current concepts in photoreceptor physiology. Physiologist 28, 122-128 (1985).

270. Hagins, W. A., Penn, R. D. \& Yoshikami, S. Dark Current and Photocurrent in Retinal Rods. Biophysical Journal 10, 380-412 (1970).

271. Capovilla, M., Cervetto, L. \& Torre, V. The effect of phosphodiesterase inhibitors on the electrical activity of toad rods. The Journal of Physiology (1983). doi:10.1111/(ISSN)1469-7793

272. Pugh, E. N. \& Cobbs, W. H. Visual transduction in vertebrate rods and cones: a tale of two transmitters, calcium and cyclic GMP. Vision Res. 26, 1613-1643 (1986).

273. Walz, B. Enhancement of sensitivity in photoreceptors of the honey been drone by light and by Ca2+. J. Comp. Physiol. A 170, 605-613 (1992).

274. Lisman, J. E. \& Brown, J. E. Effects of intracellular injection of calcium buffers on light adaptation in Limulus ventral photoreceptors. J. Gen. Physiol. 66, 489506 (1975).

275. Demer, L. L. Vascular calcification and osteoporosis: inflammatory responses to oxidized lipids. Int J Epidemiol 31, 737-741 (2002).

276. Doherty, T. M. et al. Calcification in atherosclerosis: bone biology and chronic inflammation at the arterial crossroads. Proc. Natl. Acad. Sci. U.S.A. 100, 1120111206 (2003).

277. Brown, E. M. \& MacLeod, R. J. Extracellular calcium sensing and extracellular calcium signaling. Physiological Reviews 81, 239-297 (2001).

278. Stokes, B. T., Fox, P. \& Hollinden, G. Extracellular calcium activity in the injured spinal cord. Exp. Neurol. 80, 561-572 (1983).

279. Chang, T. T.-L., Dr Kenneth Joseph Ciuffreda OD, P. \& Kapoor, N. Critical flicker frequency and related symptoms in mild traumatic brain injury. Brain Inj 21, 1055-1062 (2009).

280. Hosoya, T., Baccus, S. A. \& Meister, M. Dynamic predictive coding by the retina. Nature 436, 71-77 (2005).

281. Cook, P. B. \& McReynolds, J. S. Lateral inhibition in the inner retina is important for spatial tuning of ganglion cells. Nat Neurosci 1, 714-719 (1998).

282. Werblin, F. S. \& Copenhagen, D. R. Control of Retinal Sensitivity III. Lateral Interactions at the Inner Plexiform Layer. J. Gen. Physiol. 63, 88-110 (1974).

283. Freed, M. A., Smith, R. G. \& Sterling, P. Rod bipolar array in the cat retina: Pattern of input from rods and GABA-accumulating amacrine cells. J. Comp. Neurol. 266, 445-455 (1987).

284. Sieving, P. A., Murayama, K. \& Naarendorp, F. Push-pull model of the primate photopic electroretinogram: a role for hyperpolarizing neurons in shaping the $\mathrm{b}$ wave. Vis. Neurosci. 11, 519-532 (1994). 
285. Ohm, G. S. Die galvanische Kette, mathematisch bearbeitet. (1827).

286. Holder, D. Appendix A. Brief introduction to bioimpedance. Electrical Impedance Tomography: Methods (2004).

287. Schwan, H. P. \& Kay, C. F. Specific Resistance of Body Tissues. Circulation Research 4, 664-670 (1956).

288. Arden, G. B. \& Brown, K. T. Some properties of components of the cat electroretinogram revealed by local recording under oil. The Journal of Physiology 176, 429-461 (1965).

289. Brindley, G. S. The passive electrical properties of the frog's retina, choroid and sclera for radial fields and currents. The Journal of Physiology 134, 339-352 (1956).

290. Mohapatra, S. N. \& Hill, D. W. The changes in blood resistivity with haematocrit and temperature. Europ. J. Intensive Care Med 1, 153-162 (1975).

291. Chilbert, M. et al. Measure of tissue resistivity in experimental electrical burns. $J$ Trauma 25, 209-215 (1985).

292. Werner, C. \& Engelhard, K. Pathophysiology of traumatic brain injury. Br. J. Anaesth. 99, 4-9 (2007).

293. Gazendam, J., Go, K. G. \& van Zanten, A. K. Composition of isolated edema fluid in cold-induced brain edema. J. Neurosurg. 51, 70-77 (1979).

294. Hoffmann, K. P. \& Fischer, W. H. Directional effect of inactivation of the nucleus of the optic tract on optokinetic nystagmus in the cat. Vision Res. (2001).

295. Giolli, R. A., Blanks, R. H. I. \& Lui, F. The accessory optic system: basic organization with an update on connectivity, neurochemistry, and function. Prog. Brain Res. 151, 407-440 (2006).

296. Yoshida, K. et al. A key role of starburst amacrine cells in originating retinal directional selectivity and optokinetic eye movement. Neuron 30, 771-780 (2001).

297. Byun, H. et al. Molecular features distinguish ten neuronal types in the mouse superficial superior colliculus. - PubMed - NCBI. J. Comp. Neurol. n/a-n/a (2015). doi:10.1002/cne.23952

298. Paré, M., Crommelinck, M. \& Guitton, D. Gaze shifts evoked by stimulation of the superior colliculus in the head-free cat conform to the motor map but also depend on stimulus strength and fixation activity. Exp Brain Res 101, 123-139 (1994).

299. Tang, J., Ardila Jimenez, S. C., Chakraborty, S. \& Schultz, S. R. Visual Receptive Field Properties of Neurons in the Mouse Lateral Geniculate Nucleus. PLoS ONE 11, e0146017 (2016).

300. Glickfeld, L. L., Histed, M. H. \& Maunsell, J. H. R. Mouse primary visual cortex is used to detect both orientation and contrast changes. $J$ Neurosci 33, 1941619422 (2013).

301. Piscopo, D. M., El-Danaf, R. N., Huberman, A. D. \& Niell, C. M. Diverse visual features encoded in mouse lateral geniculate nucleus. J Neurosci 33, 4642-4656 (2013).

302. Flandrin, J. M. \& Jeannerod, M. Effects of unilateral superior colliculus ablation on oculomotor and vestibulo-ocular responses in the cat. Exp Brain Res 42, 7380 (1981). 
303. Smith, S. A. \& Bedi, K. S. Unilateral eye enucleation in adult rats causes neuronal loss in the contralateral superior colliculus. Journal of Anatomy 190, 481-490 (1997).

304. Bayly, P. V. et al. Deformation of the human brain induced by mild acceleration. J Neurotrauma 22, 845-856 (2005).

305. Gupta, R. K. \& Przekwas, A. Mathematical models of blast-induced TBI: current status, challenges, and prospects. Front. Neur. 4, (2013).

306. Hua, Y., Kumar Akula, P., Gu, L., Berg, J. \& Nelson, C. A. Experimental and Numerical Investigation of the Mechanism of Blast Wave Transmission Through a Surrogate Head. J. Comput. Nonlinear Dynam. 9, 031010 (2014).

307. Colello, S. J. \& Guillery, R. W. The changing pattern of fibre bundles that pass through the optic chiasm of mice. Eur. J. Neurosci. 10, 3653-3663 (1998).

308. Armstrong, R. C., Mierzwa, A. J., Marion, C. M. \& Sullivan, G. M. White matter involvement after TBI: Clues to axon and myelin repair capacity. Exp. Neurol. 275 Pt 3, 328-333 (2016).

309. Ramírez, A. I. et al. Macro- and microglial responses in the fellow eyes contralateral to glaucomatous eyes. Prog. Brain Res. 220, 155-172 (2015).

310. Shen, J., Fang, W., Jin, X.-H., Yao, Y.-F. \& Li, Y.-M. Sympathetic ophthalmia caused by a severe ocular chemical burn: a case report and literature review. Int $J$ Clin Exp Med 8, 2974-2978 (2015).

311. Bullock, J. D. \& Galst, J. M. The story of Louis Braille. Archives of ophthalmology (Chicago, Ill. : 1960) 127, 1532-1533 (2009).

312. Kaplan, H. J., Waldrep, J. C., Chan, W. C., Nicholson, J. K. A. \& Wright, J. D. Human Sympathetic Ophthalmia: Immunologic Analysis of the Vitreous and Uvea. Arch. Ophthalmol. 104, 240-244 (1986).

313. Cen, L. P. et al. Bilateral retinal microglial response to unilateral optic nerve transection in rats. Neuroscience 311, 56-66 (2015).

314. Bodeutsch, N., Siebert, H. \& Dermon, C. Unilateral injury to the adult rat optic nerve causes multiple cellular responses in the contralateral site. $J$ Neuroinflammation (1999).

315. Macharadze, T. et al. Interretinal transduction of injury signals after unilateral optic nerve crush. NeuroReport 20, 301-305 (2009).

316. Panagis, L., Thanos, S., Fischer, D. \& Dermon, C. R. Unilateral optic nerve crush induces bilateral retinal glial cell proliferation. Eur. J. Neurosci. 21, 2305-2309 (2005).

317. Martinez, F. O. \& Gordon, S. The M1 and M2 paradigm of macrophage activation: time for reassessment. F1000Prime Rep 6, 13 (2014).

318. $\mathrm{Hu}, \mathrm{X}$. et al. Microglial and macrophage polarization- new prospects for brain repair. Nat Rev Neurol 11, 56-64 (2015).

319. Moore, K. J., Sheedy, F. J. \& Fisher, E. A. Macrophages in atherosclerosis: a dynamic balance. Nat. Rev. Immunol. 13, 709-721 (2013).

320. Sironi, M. et al. Differential regulation of chemokine production by Fc $\gamma$ receptor engagement in human monocytes: association of CCL1 with a distinct form of M2 monocyte activation (M2b, Type 2). J. Leukoc. Biol. 80, 342-349 (2006).

321. Bell-Temin, H. et al. Novel Molecular Insights into Classical and Alternative Activation States of Microglia as Revealed by Stable Isotope Labeling by Amino 
Acids in Cell Culture (SILAC)-based Proteomics. Mol Cell Proteomics 14, 3173-3184 (2015).

322. Hao, N.-B. et al. Macrophages in Tumor Microenvironments and the Progression of Tumors. Clinical and Developmental Immunology 2012, 1-11 (2012).

323. Mackie, K. Cannabinoid receptors as therapeutic targets. Annu. Rev. Pharmacol. Toxicol. 46, 101-122 (2006).

324. Svíženská, I., Dubový, P. \& Šulcová, A. Cannabinoid receptors 1 and 2 (CB1 and $\mathrm{CB} 2$ ), their distribution, ligands and functional involvement in nervous system structures - A short review. Pharmacology Biochemistry and Behavior 90, 501-511 (2008).

325. Atwood, B. K. \& Mackie, K. CB2: a cannabinoid receptor with an identity crisis. Br. J. Pharmacol. 160, 467-479 (2010).

326. Van Sickle, M. D. et al. Identification and Functional Characterization of Brainstem Cannabinoid CB2 Receptors. Science 310, 329-332 (2005).

327. Gong, J.-P. et al. Cannabinoid CB2 receptors: Immunohistochemical localization in rat brain. Brain Res. 1071, 10-23 (2006).

328. Straiker, A., Lu, Q. \& Maguire, G. Expression of CB2 cannabinoid receptor mRNA in adult rat retina. Vis. Neurosci. 17, 91-95 (2000).

329. Onaivi, E. S. Neuropsychobiological Evidence for the Functional Presence and Expression of Cannabinoid CB2 Receptors in the Brain. Neuropsychobiology 54, 231-246 (2006).

330. Benyó, Z., Ruisanchez, É., Leszl-Ishiguro, M., Sándor, P. \& Pacher, P. Endocannabinoids in Cerebrovascular Regulation. Am. J. Physiol. Heart Circ. Physiol. ajpheart.00571.2015 (2016). doi:10.1152/ajpheart.00571.2015

331. Dowie, M. J., Grimsey, N. L., Hoffman, T., Faull, R. L. M. \& Glass, M. Cannabinoid receptor CB2 is expressed on vascular cells, but not astroglial cells in the post-mortem human Huntington's disease brain. Journal of Chemical Neuroanatomy 59-60, 62-71 (2014).

332. Rajesh, M. et al. CB2-receptor stimulation attenuates TNF- $\alpha$-induced human endothelial cell activation, transendothelial migration of monocytes, and monocyte-endothelial adhesion. Am. J. Physiol. Heart Circ. Physiol. 293, $\mathrm{H} 2210-\mathrm{H} 2218$ (2007).

333. Horváth, B. et al. A new cannabinoid CB2 receptor agonist HU-910 attenuates oxidative stress, inflammation and cell death associated with hepatic ischaemia/reperfusion injury. Br. J. Pharmacol. 165, 2462-2478 (2012).

334. Izzo, A. A., Borrelli, F., Capasso, R., Di Marzo, V. \& Mechoulam, R. Nonpsychotropic plant cannabinoids: new therapeutic opportunities from an ancient herb. Trends in Pharmacological Sciences 30, 515-527 (2009).

335. Pamplona, F. A. et al. Anti-inflammatory lipoxin A4 is an endogenous allosteric enhancer of CB1 cannabinoid receptor. Proc Natl Acad Sci USA 109, 2113421139 (2012).

336. Hind, W. H., England, T. J. \& O'Sullivan, S. E. Cannabidiol protects an in vitro model of the blood-brain barrier from oxygen-glucose deprivation via PPAR $\gamma$ and 5-HT1A receptors. Br. J. Pharmacol. 173, 815-825 (2016).

337. Presley, C. S., Mustafa, S. M., Abidi, A. H. \& Moore, B. M. Synthesis and biological evaluation of (3', 5' -dichloro-2,6-dihydroxy-biphenyl-4-yl)-aryl/alkyl- 
methanone selective CB2 inverse agonist. Bioorg. Med. Chem. 23, 5390-5401 (2015).

338. Zhu, L. X. et al. $\Delta$-9-Tetrahydrocannabinol Inhibits Antitumor Immunity by a CB2 Receptor-Mediated, Cytokine-Dependent Pathway. J. Immunol. 165, $373-$ 380 (2000).

339. Davis, E. Terence McKenna 's Last Trip Looking back at the altered statesman's psychedelic techno rap. (WIRED-San Francisco, 2000).

340. Bach, M. \& Hoffmann, M. B. Update on the Pattern Electroretinogram in Glaucoma. Optometry \& Vision Science 85, 386-395 (2008).

341. Sakatani, T. \& Isa, T. PC-based high-speed video-oculography for measuring rapid eye movements in mice. Neuroscience Research 49, 123-131 (2004).

342. Morin, L. P. \& Studholme, K. M. Retinofugal Projections in the Mouse. J. Comp. Neurol. (2014). doi:10.1002/cne.23635

343. Hammer, S. et al. Nuclei-specific differences in nerve terminal distribution, morphology, and development in mouse visual thalamus. Neural Dev 9, 16 (2014).

344. Williams, R. W. \& Chalupa, L. M. An analysis of axon caliber within the optic nerve of the cat: evidence of size groupings and regional organization. $J$ Neurosci 3, 1554-1564 (1983). 


\section{VITA}

Natalie Guley was born in Austin, Texas in 1985. After spending her teenage years away from her home city, she finally returned to finish her undergraduate education at St. Edward's University in 2007, receiving a Bachelor of Arts majoring in Psychology. In 2011, she entered medical school at the University of Tennessee Health Science Center to pursue her joint M.D./Ph.D. program. After completing her first two years of medical school, in May of 2013 she began her graduate studies in the College of Graduate Health Sciences, and received her Ph.D. in August of 2016 with a focus on Neuroscience. She will earn her M.D. degree from the College of Medicine in May of 2018, and will continue on to complete a residency training program. 